\title{
Unions, intersections and a one-shot quantum joint typicality lemma
}

\author{
PRANAB SEN ${ }^{1, *}$ \\ ${ }^{1}$ School of Technology and Computer Science, Tata Institute of Fundamental Research, Mumbai 400005, India. \\ Email: pranab.sen.73@gmail.com
}

\begin{abstract}
A fundamental tool to prove inner bounds in classical network information theory is the so-called 'conditional joint typicality lemma'. In addition to the lemma, one often uses unions and intersections of typical sets in the inner bound arguments without so much as giving them a second thought. These arguments do not work in the quantum setting. This bottleneck shows up in the fact that so-called 'simultaneous decoders', as opposed to 'successive cancellation decoders', are known for very few channels in quantum network information theory. Another manifestation of this bottleneck is the lack of so-called 'simultaneous smoothing' theorems for quantum states.

In this paper, we overcome the bottleneck by proving for the first time a one-shot quantum joint typicality lemma with robust union and intersection properties. To do so, we develop two novel tools in quantum information theory which may be of independent interest. The first tool is a simple geometric idea called tilting, which increases the angles between a family of subspaces in orthogonal directions. The second tool, called smoothing and augmentation, is a way of perturbing a multipartite quantum state such that the partial trace over any subset of registers does not increase the operator norm by much.

Our joint typicality lemma allows us to construct simultaneous quantum decoders for many multiterminal quantum channels. It provides a powerful tool to extend many results in classical network information theory to the one-shot quantum setting.
\end{abstract}

Keywords. quantum simultaneous decoder; joint typicality; one-shot inner bounds; multiple access channel; network information theory.

\section{Introduction}

A fundamental tool to prove inner bounds for communication channels in classical network information theory is the so-called conditional joint typicality lemma [1]. Very often, the joint typicality lemma is used together with implicit intersection and union arguments in the inner bound proofs. This is especially so in the construction of so-called simultaneous decoders (as opposed to successive cancellation decoders) for communication problems. In this paper, we investigate what happens when one tries to extend the classical inner bound proofs to the quantum setting. It turns out that the union and intersection arguments present a huge stumbling block in this effort. The main result of this paper is a oneshot quantum joint typicality lemma that takes care of these union and intersection bottlenecks, and allows us to extend many classical inner bound proofs to the quantum setting.

\subsection{Information theory and typical sequences}

In a path breaking paper [2], Shannon started the field of information theory. The paper introduced several revolutionary ideas viz:

- Abstracting out a noisy communication channel with one input and one output, henceforth referred to as a point-to-point channel, in a very general fashion as a probability transition matrix that maps a symbol from its input alphabet, e.g. $\{0,1\}$, into a probability dis- tribution over symbols from its output alphabet, e.g. $\{0,1\}$;

- Showing that it is possible to reduce the error of transmitting messages over a noisy channel by introducing some redundancy by encoding the message first, inputting the encoded message to the channel, and then decoding the output in an attempt to recover the sent message;

- Shifting the onus of reliable information transmission away from the engineering approach of reducing the noise in a channel, which was the one emphasised earlier, to the mathematical approach of constructing better codes so that higher communication rate and lower error rate could simultaneously be achieved using the physical same channel as before;

- Showing that with many independent and identical uses of the channel, it is possible to simultaneously achieve larger rates of information transmission per channel use as well as smaller information transmission error;

- Defining a precise mathematical quantity called channel capacity which is purely a function of the channel's probability transition matrix that has the the property that, in the limit of many independent and identical uses of the channel, almost error free transmission of messages at any rate below the capacity is possible, 
and any strategy for transmitting at a rate larger than the capacity necessarily incurs non-vanishing error.

The last item above, viz. the channel capacity, was defined in terms of a quantity called mutual information, which in turn was defined in terms of a novel quantity informationtheoretic entropy inspired by the concept of thermodynamic entropy of physics. Shannon's noisy channel coding theorem that proved the above property regarding channel capacity again introduced a new concept called typical sequence that formalised the famous law of large numbers from probability theory in the following particular way: in any sequence of samples of length $n$ drawn independently from a probability distribution $p(\cdot)$ on a fixed finite alphabet $\mathcal{X}$, with high probability, the number of occurences in the sequence of every symbol $x \in \mathcal{X}$ is roughly $n p(x)$. Such sequences are said to be typical with respect to the probability distribution $p(x)$.

The concept of a typical sequence naturally leads to the concept of a jointly typical sequence with respect to a probability distribution $p(\cdot)$ on a fixed finite set $\mathcal{X} \times \mathcal{Y}$. Thus, a sequence $\left(\left(x_{1}, y_{1}\right), \ldots,\left(x_{n}, y_{n}\right)\right)$ of length $n$ is said to be $\delta$-jointly typical with respect to $p(x, y)$ iff the number of occurences of each symbol $(x, y) \in \mathcal{X} \times \mathcal{Y}$ is in the range $n p(x, y)(1 \pm \delta)$. Often proofs in Shannon theory investigate questions like, e.g.,

Consider a sequence $\left(\left(x_{1}, y_{1}\right), \ldots,\left(x_{n}, y_{n}\right)\right)$ drawn independently from the probability distribution $p(x) p(y)$, which is nothing but the product of the marginal distributions on $\mathcal{X}$ and $\mathcal{Y}$. What is the probability that it is $\delta$-jointly typical with respect to $p(x, y)$ ?

The answers to these types of questions are provided by socalled joint typicality lemmas. They form a cornerstone of techniques to prove various inner bounds, i.e. achievability results, in channel coding. Often these proofs require one to take intersections and unions of sets of typical sequences. This is especially so when one studies inner bounds for multiterminal channels, i.e. channels with more than one input and/or more than one output.

As long as one is dealing only with classical channels i.e. channels where information is carried by properties of physical systems behaving according to non-quantum physics, Shannon's mathematical abstraction of channels as explained above works without any problem. Typical sets and intersections and unions provide a well oiled machinery to attack channel coding problems in the limit of many independent and identical uses of the channel, called asymptotic iid setting henceforth. Classical channels encompass the whole gamut of communication channels in current use, ranging from wireless systems to optical fibres. The story however radically changes when one considers the quantum setting where information is carried by properties of physical systems behaving according to the laws of quantum physics. Examples of quantum channels include using photon polarisation to transmit information over optical fibre, using nuclear spins in organic molecules to store information etc. It is this setting that we study in this paper.
More than a decade before Deutsch published the first mathematical model of a quantum computer [3], Holevo studied the information carrying capabilities of quantum systems in his seminal paper [4]. Interest in quantum computing and quantum information theory grew exponentially after Shor's publication of an efficient quantum algorithm for integer factoring [5]. Barely a few years after Shor's result, Holevo [6] and Schumacher-Westmoreland [7] proved a quantum analogue of Shannon's noisy channel coding theorem by showing that the quantum version of mutual information is a lower bound on the capacity of a quantum channel to carry classical information in the asymptotic iid setting. In doing so, they generalised the notion of a typical set to a typical subspace which, in hindsight, was the natural thing to do since decoding operations in the quantum setting are mathematically modelled by projections onto subspaces.

As long as one is working only with point-to-point channels, many inner bounds in asymptotic iid classical information theory can be extended to the quantum setting by replacing typical sets with typical subspaces and classical joint typicality lemmas with quantum joint typicality lemmas. However as soon as one starts to deal with multiterminal channels, one encounters the issue of defining intersections and unions of typical subspaces which becomes a significant bottleneck. The situation becomes worse when one has to deal with multiterminal quantum channels in the one shot setting where the channel can be used only once. Though analogues for typical sets and typical subspaces have recently been developed for the one shot setting, see e.g [8], their properties are not as nice as those for the asymptotic iid setting. This exacerbates the bottleneck of defining unions and intersections. Though some ad hoc solutions have been found for some quantum channels in the asymptotic iid regime, see e.g. [9, [10], this bottleneck continues to be a hindrance in proving good inner bounds for multiterminal quantum channels in general.

In the next few subsections we will explain the problem of intersections and unions of typical subspaces in more detail by taking up the example of the simplest multiterminal quantum channel viz. the multiple access channel (MAC). We will first explicitly show why an intersection argument arises while proving an inner bound for the classical MAC in the one shot setting. We will then explain why naive strategies fail to address the intersection issue for the quantum MAC. After that we will intuitively describe the two new tools introduced in this paper called tilting and smoothing and augmentation, and then explain how they can be used to define a robust notion of intersection and union of typical subspaces that finally overcomes the bottleneck in the quantum setting. The tools developed in this paper all work in the most general one shot quantum setting.

\subsection{One-shot inner bound for the classical MAC}

Let us illustrate the need for an intersection argument together with a joint typicality lemma by considering the problem of proving inner bounds for arguably the simplest multiterminal communication channel viz. the multiple access 
channel (MAC) (see Fig. 1). We consider the one-shot classical setting. There are two senders Alice and Bob who would like to send messages $m_{1} \in\left[2^{R_{1}}\right], m_{2} \in 2^{R_{2}}$, respectively to a receiver Charlie. There is a communication channel $\mathfrak{C}$ with two inputs and one output called the two-sender one-receiver MAC connecting Alice and Bob to Charlie. The two input alphabets of $\mathbb{C}$ will be denoted by $\mathcal{X}, \mathcal{Y}$ and the output alphabet by $\mathcal{Z}$. Let $0 \leq \epsilon \leq 1$. On getting message $m_{1}$, Alice encodes it as a letter $x\left(m_{1}\right) \in \mathcal{X}$ and feeds it to her channel input. Similarly on getting message $m_{2}$, Bob encodes it as a letter $y\left(m_{2}\right) \in \mathcal{Y}$ and feeds it to his channel input. The channel $\mathfrak{C}$ outputs a letter $z \in \mathcal{Z}$ according to the channel probability distribution $p\left(z \mid x\left(m_{1}\right), y\left(m_{2}\right)\right)$. Charlie now has to try and guess the message pair $\left(m_{1}, m_{2}\right)$ from the channel output. We require that the probability of Charlie's decoding error, where the probability arises both from Charlie's measurement as well as classically averaging over the uniform distribution on the set of message pairs $\left(m_{1}, m_{2}\right) \in\left[2^{R_{1}}\right] \times\left[2^{R_{2}}\right]$, is at most $\epsilon$.

Consider the following randomised construction of a codebook $C$ for Alice and Bob. Fix probability distributions $p(x)$, $p(y)$ on sets $\mathcal{X}, \mathcal{Y}$. For $m_{1} \in\left[2^{R_{1}}\right]$, choose $x\left(m_{1}\right) \in \mathcal{X}$ independently according to $p(x)$. Similarly for $m_{2} \in\left[2^{R_{2}}\right]$, choose $y\left(m_{2}\right) \in \mathcal{Y}$ independently according to $p(y)$.

Before describing the decoding strategy that Charlie follows, it is useful to first define a concept called hypothesis testing. Let $0 \leq \epsilon \leq 1$. Let $p, q$ be two probability distrbutions on the same sample space $\Omega$. A 'classical POVM element' or 'test' on $\Omega$ is defined to be a function $f: \Omega \rightarrow$ $[0,1]$. Intuitively, for a sample point $\omega \in \Omega, f(\omega)$ denotes its probability of acceptance by the test. For two classical POVM elements $f, g$ on $\Omega$, we can define the 'intersection' classical POVM element $f \cap g$ as follows: $(f \cap g)(\omega):=$ $\min \{f(\omega), g(\omega)\}$. Similarly, we can define the 'union' classical POVM element $f \cup g$ as follows: $(f \cup g)(\omega):=\max \{f(\omega), g(\omega)\}$. Following Wang and Renner [8], we define the classical hypothesis testing relative entropy $D_{H}^{\epsilon}(p \| q)$ as follows:

$$
D_{H}^{\epsilon}(p \| q):=\max _{f: \sum_{\omega} f(\omega) p(\omega) \geq 1-\epsilon}-\log \sum_{\omega} f(\omega) q(\omega),
$$

where the maximisation is over all classical POVM elements on $\Omega$ 'accepting' the distribution $p$ with probability at least $1-\epsilon$. It is easy to see that the optimising POVM element $f$ attains equality in the constraint for $p(\cdot)$, as well as achieves the maximum in objective function for $q(\cdot)$.

Define the probability distribution $p(\cdot \mid x)$ on $\mathcal{Z}$ as $p(z \mid x):=$ $\sum_{y} p(y) p(z \mid x, y)$ for all $z \in \mathcal{Z}$. With a slight abuse of notation, we shall often use $p(z \mid x)$ to also denote the distribution $p(\cdot \mid x)$ on $\mathcal{Z}$. Similarly, define probability distributions $p(\cdot \mid y), p(\cdot)$ on $\mathcal{Z}$ in the natural fashion. Define the probability distributions

$$
\begin{aligned}
p^{X Y Z}(x, y, z) & :=p(x) p(y) p(z \mid x, y), \\
\left(p^{X Z} \times p^{Y}\right)(x, y, z) & :=p(x) p(y) p(z \mid x), \\
\left(p^{Y Z} \times p^{X}\right)(x, y, z) & :=p(x) p(y) p(z \mid y), \\
\left(p^{X} \times p^{Y} \times p^{Z}\right)(x, y, z) & :=p(x) p(y) p(z)
\end{aligned}
$$

on $\mathcal{X} \times \mathcal{Y} \times \mathcal{Z}$ in the natural manner. Consider classical POVM elements $f^{Y}, f^{X}, f^{X, Y}$ achieving the respective maxima in the definitions of $D_{H}^{\epsilon}\left(p^{X Y Z} \| p^{X Z} \times p^{Y}\right), D_{H}^{\epsilon}\left(p^{X Y Z} \| p^{Y Z} \times p^{X}\right)$, $D_{H}^{\epsilon}\left(p^{X Y Z} \| p^{X} \times p^{Y} \times p^{Z}\right)$ respectively. As a shorthand, we will use the hypothesis testing mutual information quantities $I_{H}^{\epsilon}(Y: X Z), I_{H}^{\epsilon}(X: Y Z), I_{H}^{\epsilon}(X Y: Z)$ to denote the hypothesis testing relative entropy quantities $D_{H}^{\epsilon}\left(p^{X Y Z} \| p^{X Z} \times p^{Y}\right)$, $D_{H}^{\epsilon}\left(p^{X Y Z} \| p^{Y Z} \times p^{X}\right), D_{H}^{\epsilon}\left(p^{X Y Z} \| p^{X} \times p^{Y} \times p^{Z}\right)$ respectively. Thus,

$$
\begin{aligned}
\sum_{x, y, z} p(x, y, z) f^{X}(x, y, z) & \geq 1-\epsilon, \\
\sum_{x, y, z} p(x) p(y, z) f^{X}(x, y, z) & \leq 2^{-I_{H}^{\epsilon}(X: Y Z)} ; \\
\sum_{x, y, z} p(x, y, z) f^{Y}(x, y, z) & \geq 1-\epsilon, \\
\sum_{x, y, z} p(y) p(y, z) f^{Y}(x, y, z) & \leq 2^{-I_{H}^{\epsilon}(Y: X Z)} ; \\
\sum_{x, y, z} p(x, y, z) f^{X, Y}(x, y, z) & \geq 1-\epsilon, \\
\sum_{x, y, z} p(x) p(y) p(z) f^{X, Y}(x, y, z) & \leq 2^{-I_{H}^{\epsilon}(X Y: Z) .}
\end{aligned}
$$

We now describe the decoding strategy that Charlie follows in order to try and guess the message pair $\left(m_{1}, m_{2}\right)$ that was actually sent. Consider the intersection classical POVM element $f:=f^{X} \cap f^{Y} \cap f^{X, Y}$. Suppose the channel output is $z$. Then, Charlie uses the following randomised algorithm for decoding.

$$
\begin{aligned}
& \text { For } \hat{m}_{1}=1 \text { to } 2^{R_{1}} \\
& \text { For } \hat{m}_{2}=1 \text { to } 2^{R_{2}} \\
& \text { Toss a coin with probability of HEAD } \\
& \quad \text { being } f\left(x\left(\hat{m}_{1}\right), y\left(\hat{m}_{2}\right), z\right) .
\end{aligned}
$$

If the coin comes up HEAD, declare $\left(\hat{m}_{1}, \hat{m}_{2}\right)$ as Charlie's guess and halt.

$$
\text { If the coin comes up TAILS, go to }
$$$$
\text { next iteration. }
$$

Declare FAIL, if Charlie did not declare any guess above.

We now analyse the expectation, under the choice of a random codebook $C$, of the error probability of Charlie's decoding algorithm. Suppose the message pair $\left(m_{1}, m_{2}\right)$ is inputted to the channel. Let $\prec$ denote the lexicographic order on message pairs. Let the channel output be denoted by $z$. Then, a decoding error occurs only if Charlie tosses a HEAD for a pair $\left(\hat{m}_{1}, \hat{m}_{2}\right) \prec\left(m_{1}, m_{2}\right)$ or if Charlie tosses a TAIL for $\left(m_{1}, m_{2}\right)$. The expectation, over the choice of the random codebook $C$, of the decoding error is then upper bounded by

$$
\begin{aligned}
& \underset{C}{\mathbf{E}}\left[p_{e}\left(C ; m_{1}, m_{2}\right)\right] \\
& \leq \quad 2^{R_{1}+R_{2}} 2^{-I_{H}^{\epsilon}(X Y: Z)}+2^{R_{2}} 2^{-I_{H}^{\epsilon}(Y: X Z)} \\
& \quad+2^{R_{1}} 2^{-I_{H}^{\epsilon}(X: Y Z)}+3 \epsilon .
\end{aligned}
$$

A proof can be found in Appendix II. Now define the average decoding error probability under a codebook $C$ to be

$$
p_{e}(C):=2^{-\left(R_{1}+R_{2}\right)} \sum_{\left(m_{1}, m_{2}\right)} p_{e}\left(C ; m_{1}, m_{2}\right) .
$$




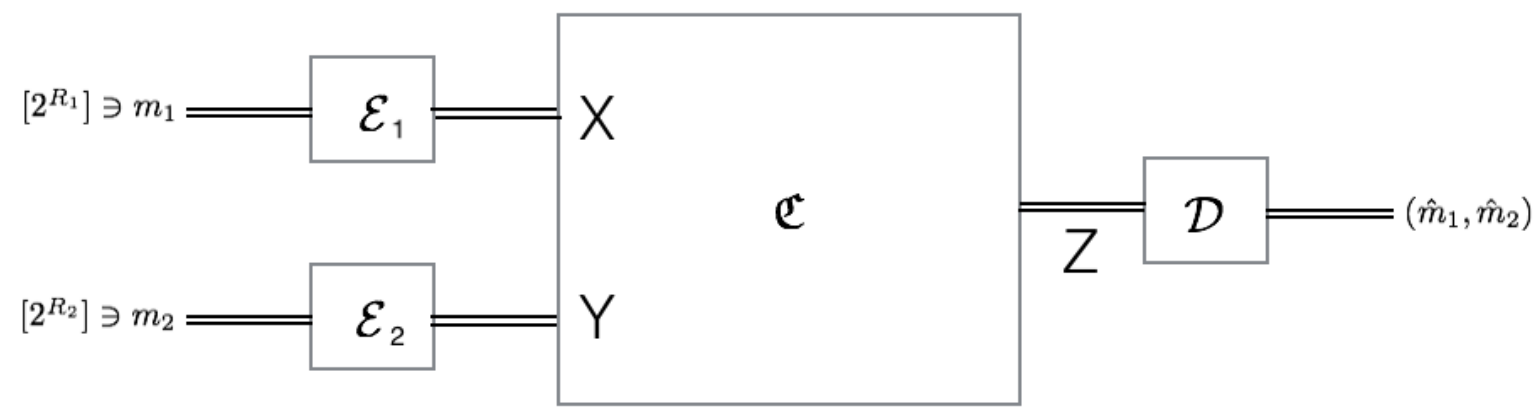

Figure 1. A classical multiple access channel. Alice, Bob encode their respective messages $m_{1}, m_{2}$ and then feed the resulting codewords to the channel. Charlie decodes to get his guess $\left(\hat{m}_{1}, \hat{m}_{2}\right)$ of the transmitted messages. $X, Y$ are the input alphabets and $Z$ is the output alphabet of the channel.

Then the expectation, under the choice of a random codebook $C$, of the average decoding error probability is upper bounded by

$\underset{C}{\mathbf{E}}\left[p_{e}(C)\right] \leq 2^{R_{1}+R_{2}} 2^{-I_{H}^{\epsilon}(X Y: Z)}+2^{R_{2}} 2^{-I_{H}^{\epsilon}(Y: X Z)}+2^{R_{1}} 2^{-I_{H}^{\epsilon}(X: Y Z)}+3 \epsilon$.

Thus, for any rate pair in the region given by

$$
\begin{aligned}
R_{1} & \leq I_{H}^{\epsilon}(X: Y Z)-\log \frac{1}{\epsilon}, \\
R_{2} & \leq I_{H}^{\epsilon}(Y: X Z)-\log \frac{1}{\epsilon}, \\
R_{1}+R_{2} & \leq I_{H}^{\epsilon}(X Y: Z)-\log \frac{1}{\epsilon},
\end{aligned}
$$

there is a codebook $C$ with average decoding error probability less than $6 \epsilon$.

\subsection{Unions and intersections in the classical setting}

We now step back and discuss the intersection classical POVM element $f:=f^{X} \cap f^{Y} \cap f^{X, Y}$ used in the above proof. The intersection argument was crucial in constructing a simultaneous decoder for Charlie. In the asymptotic iid setting for the multiple access channel, it is possible to avoid simultaneous decoding and instead use successive cancellation decoding combined with time sharing [1]. However, in the oneshot setting time sharing does not make sense and successive cancellation gives only a finite set of achievable rate pairs. Thus, in order to get a continuous achievable rate region, we are forced to use simultaneous decoders only. There are also situations even in the asymptotic iid setting, e.g. in Marton's inner bound with common message for the broadcast channel, where we need to use intersection arguments [1]. Similarly, union arguments crop up in some inner bound proofs, e.g. Marton's inner bound without common message for the broadcast channel, even in the asymptotic iid setting [1]. In the one-shot setting, union bounds occur more frequently e.g., in the Han-Kobayashi inner bound for the interference channel [11]. Thus, intersection and union arguments are indispensable in network information theory.

Before we proceed to the quantum setting, we prove for completeness sake a 'one-shot classical joint typicality lemma'. In fact, it is nothing but an application of intersection and union of classical POVM elements.

Fact 1 (Classical joint typicality lemma) Let $p_{1}, \ldots, p_{t}$, $q_{1}, \ldots, q_{l}$ be probability distributions on a set $\mathcal{X}$. Let $0 \leq$ $\left\{\epsilon_{i j}\right\}_{i j} \leq 1$, where $i \in[t], j \in[l]$. Then there is a classical POVM element $f$ on $\mathcal{X}$ such that:

$$
\begin{aligned}
& \text { 1. For all } i \in[t], \sum_{x} p_{i}(x) f(x) \geq 1-\left\{\sum_{j=1}^{l} \epsilon_{i j}\right\} ; \\
& \text { 2. For all } j \in[l], \sum_{x} q_{j}(x) f(x) \leq \sum_{i=1}^{t} 2^{-D_{H}^{\epsilon_{i j}}\left(p_{i} \| q_{j}\right)} \text {. }
\end{aligned}
$$

Proof: For $i \in[t], j \in[l]$, let $f_{i j}$ be the classical POVM element achieving the minimum in the definition of $D_{H}^{\epsilon_{i j}}\left(p_{i} \| q_{j}\right)$. Define the classical POVM element $f:=\cup_{i=1}^{t} \cap_{j=1}^{l} f_{i j}$. Observe that for any $x \in \mathcal{X}$,

$$
1-f(x) \leq \min _{i: i \in[t]}\left\{\sum_{j=1}^{l}\left(1-f_{i j}(x)\right)\right\}, \quad f(x) \leq \sum_{i=1}^{t} \min _{j: j \in[l]}\left\{f_{i j}(x)\right\} .
$$

It is now easy to see that $f$ satisfies the properties claimed above.

\subsection{Extending unions and intersections to the quantum set- ting}

We now ponder what is required to extend the above inner bound proof for the classical MAC to the setting of the oneshot classical-quantum multiple access channel (cq-MAC). In the cq-MAC, there are two senders Alice and Bob who would like to send messages $m_{1} \in\left[2^{R_{1}}\right], m_{2} \in 2^{R_{2}}$, respectively to a receiver Charlie. There is a communication channel $\mathfrak{C}$ with two classical inputs and one quantum output connecting Alice and Bob to Charlie. The two input alphabets of $\mathfrak{C}$ will be denoted by $\mathcal{X}, \mathcal{Y}$ and the output Hilbert space by $\mathcal{Z}$. If the pair $(x, y)$ is fed into the channel inputs, the output of the channel is a density matrix $\rho_{x, y}$ in $\mathcal{Z}$. Let $0 \leq \epsilon \leq 1$. On 
getting message $m_{1}$, Alice encodes it as a letter $x\left(m_{1}\right) \in \mathcal{X}$ and feeds it to her channel input. Similarly on getting message $m_{2}$, Bob encodes it as a letter $y\left(m_{2}\right) \in \mathcal{Y}$ and feeds it to his channel input. Charlie now has to try and guess the mes-

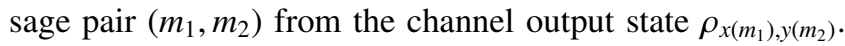
We require that the probability of Charlie's decoding error averaged over the uniform distribution on the set of message pairs $\left(m_{1}, m_{2}\right) \in\left[2^{R_{1}}\right] \times\left[2^{R_{2}}\right]$ is at most $\epsilon$.

One can do a similar randomised construction of a codebook $C$ for Alice and Bob as before. One can make use of the hypothesis testing relative entropy [8] for a pair of quantum states $\rho, \sigma$ in the same Hilbert space $\mathcal{H}$, which is defined as follows:

$$
D_{H}^{\epsilon}(\rho \| \sigma):=\max _{\Pi: \operatorname{Tr}[\Pi \rho] \geq 1-\epsilon}-\log \operatorname{Tr}[\Pi \sigma],
$$

where the maximisation is over all POVM elements $\Pi$ on $\mathcal{H}$ (i.e. positive semidefinite operators $\Pi$ such that $\Pi \leq \mathbb{1}_{\mathcal{H}}$ ) 'accepting' the state $\rho$ with probability at least $1-\epsilon$. Again, it is easy to see that the optimising POVM element $\Pi$ attains equality in the constraint for $\rho$, as well as achieves the maximum in objective function for $\sigma$. One can define the analogous quantum state

$$
\rho^{X Y Z}:=\sum_{x, y} p(x) p(y)|x\rangle\left\langle\left. x\right|^{X} \otimes \mid y\right\rangle\left\langle\left. y\right|^{Y} \otimes \rho_{x, y}^{Z}\right.
$$

and the tensor products of the marginals $\rho^{X Z} \otimes \rho^{Y}, \rho^{Y Z} \otimes \rho^{X}$, $\rho^{X} \otimes \rho^{Y} \otimes \rho^{Z}$, as well as the hypothesis testing mutual informations

$$
\begin{aligned}
I_{H}^{\epsilon}(X: Y Z) & :=D_{H}^{\epsilon}\left(\rho^{X Y Z} \| \rho^{X} \otimes \rho^{Y Z}\right), \\
I_{H}^{\epsilon}(Y: X Z) & :=D_{H}^{\epsilon}\left(\rho^{X Y Z} \| \rho^{Y} \otimes \rho^{X Z}\right), \\
I_{H}^{\epsilon}(X Y: Z) & :=D_{H}^{\epsilon}\left(\rho^{X Y Z} \| \rho^{X} \otimes \rho^{Y} \otimes \rho^{Z}\right)
\end{aligned}
$$

in the quantum setting too. Thus, we would like to prove that any rate pair in the region given by

$$
\begin{aligned}
R_{1} & \leq I_{H}^{\epsilon}(X: Y Z)-\log \frac{1}{\epsilon}, \\
R_{2} & \leq I_{H}^{\epsilon}(Y: X Z)-\log \frac{1}{\epsilon}, \\
R_{1}+R_{2} & \leq I_{H}^{\epsilon}(X Y: Z)-\log \frac{1}{\epsilon}
\end{aligned}
$$

is achievable with small average decoding error probability.

Suppose there exists a single POVM element $\Pi$ on the Hilbert space $\mathcal{X} \otimes \mathcal{Y} \otimes \mathcal{Z}$ that simultaneously satisfies the following properties:

\section{Desirable properties of the single POVM element $\Pi$}

$$
\begin{aligned}
& \text { 1. } \operatorname{Tr}\left[\Pi \rho^{X Y Z}\right] \geq 1-3 \epsilon ; \\
& \text { 2. } \operatorname{Tr}\left[\Pi\left(\rho^{Y Z} \otimes \rho^{X}\right)\right] \leq 2^{-I_{H}^{\epsilon}(X: Y Z)} ; \\
& \text { 3. } \operatorname{Tr}\left[\Pi\left(\rho^{X Z} \otimes \rho^{Y}\right)\right] \leq 2^{-I_{H}^{\epsilon}(Y: X Z)} ; \\
& \text { 4. } \operatorname{Tr}\left[\Pi\left(\rho^{X} \otimes \rho^{Y} \otimes \rho^{Z}\right)\right] \leq 2^{-I_{H}^{\epsilon}(X Y: Z)} .
\end{aligned}
$$

In the classical setting, such a POVM element $f$ was constructed by taking the intersections of the three POVM elements $f^{X}, f^{Y}, f^{X, Y}$ achieving the respective maxima in the definitions of the three entropic quantities $I_{H}^{\epsilon}(X: Y Z), I_{H}^{\epsilon}(Y$ : $X Z), I_{H}^{\epsilon}(X Y: Z)$. In the quantum setting, if such an 'intersection' POVM element $\Pi$ exists it is indeed possible to construct a decoding algorithm for Charlie with average error probability at most $O(\epsilon)$ using the 'pretty good measurement' [12, 13, 6, 7], the Hayashi-Nagaoka operator inequality [14] and mimicking the classical analysis given above for the decoding error.

Let $\Pi^{X}, \Pi^{Y}, \Pi^{X, Y}$ be the three POVM elements achieving the maxima in the definitions of the three entropic quantities $I_{H}^{\epsilon}(X: Y Z), I_{H}^{\epsilon}(Y: X Z), I_{H}^{\epsilon}(X Y: Z)$. Let us now look at various simple ideas to generalise intersection of POVM elements to the quantum setting. If the POVM elements were projectors, which can be ensured without loss of generality by the Gelfand-Naimark technique of embedding the quantum states into a larger Hilbert space $\mathcal{X} \otimes \mathcal{Y} \otimes\left(\mathcal{Z} \otimes \mathbb{C}^{2}\right)$, one can try taking the projector $\Pi$ onto the intersection of the supports of $\Pi^{X}, \Pi^{Y}, \Pi^{X, Y}$. This indeed ensures that Properties 2, 3, 4 described above hold for $\Pi$. Unfortunately, the intersection can easily be the zero subspace which kills all hope of satisfying Property 1 even approximately. The next simple idea to define the 'intersection' POVM element would be to take the product $\Pi:=\Pi^{X} \Pi^{Y} \Pi^{X, Y} \Pi^{X, Y} \Pi^{Y} \Pi^{X}$. With this definition, $\Pi$ can be shown to satisfy Property 1 with lower bound $1-O(\epsilon)$ using the non-commutative union bound [15, 16]. However, it is not at all clear that the remaining three properties can be simultaneously satisfied, even approximately, because $\Pi^{X}, \Pi^{Y}, \Pi^{X, Y}$ do not commute in general. To get an idea of the difficulty, consider the expression

$$
\operatorname{Tr}\left[\Pi\left(\rho^{Y Z} \otimes \rho^{X}\right)\right]=\operatorname{Tr}\left[\Pi^{X} \Pi^{Y} \Pi^{X, Y} \Pi^{X, Y} \Pi^{Y} \Pi^{X}\left(\rho^{Y Z} \otimes \rho^{X}\right)\right]
$$

that arises if one were to attempt to prove Property 2 . It is true that $\operatorname{Tr}\left[\Pi^{Y}\left(\rho^{Y Z} \otimes \rho^{X}\right)\right]=2^{-I(X: Y Z)}$, but it is also possible that

$$
\begin{gathered}
\operatorname{Tr}\left[\Pi^{X} \Pi^{Y} \Pi^{X, Y} \Pi^{X, Y} \Pi^{Y} \Pi^{X}\left(\rho^{Y Z} \otimes \rho^{X}\right)\right] \\
-\operatorname{Tr}\left[\Pi^{Y}\left(\rho^{Y Z} \otimes \rho^{X}\right)\right] \\
\gg 2^{-I(X: Y Z)} .
\end{gathered}
$$

This makes it impossible to show the achievability of the desired rate region (Equation 3) with this definition of $\Pi$. In fact, one of the main technical contributions of this paper is a robust novel notion of intersection of non-commuting POVM elements achieving the maxima in the definitions of the appropriate hypothesis testing mutual information quantities.

As a first step towards defining a robust notion of intersection of non-commuting POVM elements, we address the complementary problem of defining a robust notion of union of non-commuting POVM elements. The intersection' POVM element can then be defined to be simply the complement of the 'union' of the complements. Note that the complement of a POVM element $\Pi$ in a Hilbert space $\mathcal{H}$ is defined to be $\mathbb{1}_{\mathcal{H}}-\Pi$. The POVM elements are projectors without loss of generality. One can then naively define the 'union' of a family of projectors to be the projector onto the span of the supports (the union of supports is 


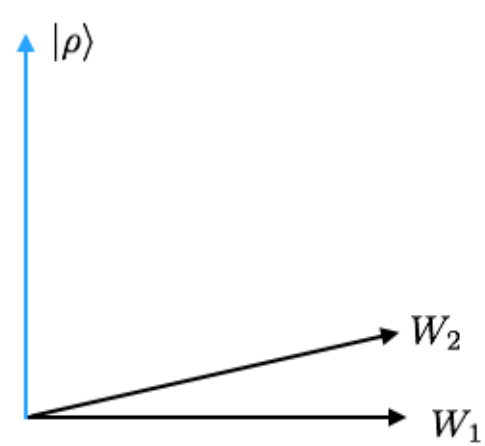

Pathology: Span can be entire space

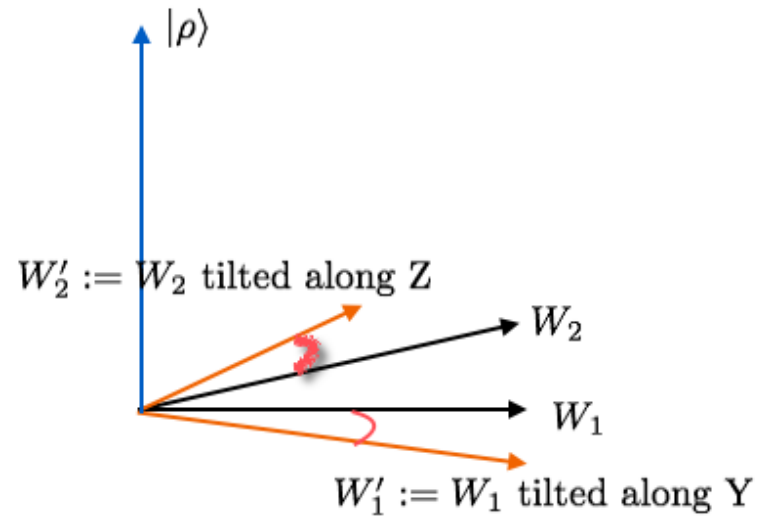

Tilting: The pathology is destroyed now!

Figure 2. Span versus tilted span. The left figure shows how a quantum state $\rho$ can have small projections on two subspaces $W_{1}$ and $W_{2}$ but large projection on their span $W_{1}+W_{2}$. The pathology can be severe, in that the state and the subspaces can all be one dimensional and the state can lie fully in the span of the two subspaces. On the right, we see how tilting the two subspaces along two orthogonal directions (which we call $\mathrm{Y}$ and $\mathrm{Z}$ in the figure) destroys the pathology. The quantum state $\rho$ now has small projection onto the span $W_{1}^{\prime}+W_{2}^{\prime}$ of the two tilted subspaces $W_{1}^{\prime}$ and $W_{2}^{\prime}$.

not a vector space in general). However, this does not give us anything new as the span can indeed be the entire space and so Property 1 can be totally useless, that is, the lower bound in Property 1 can be as low as zero! The problem with the span idea is captured by the following example. Consider the two-dimensional Hilbert space $\mathbb{C}^{2}$. Let $W_{1}$ be the one-dimensional space spanned by $|0\rangle$ and $W_{2}$ the onedimensional space spanned by $\sqrt{1-\epsilon}|0\rangle+\sqrt{\epsilon}|1\rangle$. Consider the quantum state $\rho:=|1\rangle\langle 1|$. Now the probability of $\rho$ being accepted by $W_{1}, W_{2}$ is 0 and $\epsilon$ respectively. However, the probability of $\rho$ being accepted by the span of $W_{1}$ and $W_{2}$ is one.

Notice however that the above pathological phenomenon with the span occurs only because the subspaces $W_{1}$ and $W_{2}$ have 'small angles' between them. We overcome this problem by 'tilting' $W_{1}, W_{2}$ in orthogonal directions to form new spaces $W_{1}^{\prime}, W_{2}^{\prime}$ (see Fig. 2). The Hilbert space has to be enlarged sufficiently to allow this tilting to be possible. The process of tilting increases the 'angles' between the subspaces in orthogonal directions allowing one to recover an upper bound for the span very close to the sum of acceptance probabilities, just like in the classical setting. This 'tilting' idea is formalised in Proposition 2. Thus, the 'tilted span' is best thought of as a robust notion of union of subspaces satisfying a well behaved union bound.

We now describe the tilting process for the above 'pathological' example of Fig. 2 in detail. Let $A_{1} \cong \mathbb{C}^{2}$ be the original Hilbert space. Consider the extended Hilbert space $\hat{A}:=A_{1} \oplus A_{2} \oplus A_{3}$, where each $A_{i} \cong \mathbb{C}^{2}$. The subspaces $W_{1}$, $W_{2}$ are the one-dimensional subspaces spanned by the vectors $|0\rangle^{A_{1}}$ and $\sqrt{1-\epsilon}|0\rangle^{A_{1}}+\sqrt{\epsilon}|1\rangle^{A_{1}}$ respectively, in $\hat{A}$. Fix $0<\alpha<1 / 2$. The tilted subspaces are defined by

$$
\begin{aligned}
W_{1}^{\prime}:=\operatorname{span}\left\{\sqrt{1-\alpha}|0\rangle^{A_{1}}+\sqrt{\alpha}|0\rangle^{A_{2}}\right\}, \\
W_{2}^{\prime}:=\operatorname{span}\left\{\sqrt{(1-\alpha)(1-\epsilon)}|0\rangle^{A_{1}}+\sqrt{\alpha(1-\epsilon)}|0\rangle^{A_{3}}\right. \\
\\
\left.+\sqrt{(1-\alpha) \epsilon}|1\rangle^{A_{1}}+\sqrt{\alpha \epsilon}|1\rangle^{A_{3}}\right\},
\end{aligned}
$$

where the span is taken in $\hat{A}$. Consider now the span $W^{\prime}:=$ $W_{1}^{\prime}+W_{2}^{\prime}$. We refer to $W^{\prime}$ as the 'tilted span' of $W_{1}$ and $W_{2}$. It has the following orthonormal basis:

$$
\begin{aligned}
& W^{\prime}=\operatorname{span}\left\{\sqrt{1-\alpha}|0\rangle^{A_{1}}+\sqrt{\alpha}|0\rangle^{A_{2}},\right. \\
& \alpha \sqrt{(1-\alpha)(1-\epsilon)}|0\rangle^{A_{1}}-(1-\alpha) \sqrt{\alpha(1-\epsilon)}|0\rangle^{A_{2}} \\
& \left.\frac{+\sqrt{\alpha(1-\epsilon)}|0\rangle^{A_{3}}+\sqrt{(1-\alpha) \epsilon}|1\rangle^{A_{1}}+\sqrt{\alpha \epsilon}|1\rangle^{A_{3}}}{\sqrt{\epsilon+\alpha(1-\epsilon)(2-\alpha)}}\right\} .
\end{aligned}
$$

Thus tilting destroys the pathology since the projection of $|\rho\rangle$ onto $W^{\prime}$ has length

$$
\frac{\sqrt{(1-\alpha) \epsilon}}{\sqrt{\epsilon+\alpha(1-\epsilon)(2-\alpha)}}<\frac{1}{\sqrt{\alpha}} \sqrt{\epsilon} .
$$

Suppose $\epsilon<1 / 4$. Setting $\alpha=\sqrt{\epsilon}$, we can finally conclude that the tilted subspaces are $\left(2^{1 / 2} \epsilon^{1 / 4}\right)$-close to their respective originals in the $\ell_{2}$-norm, whereas $|\rho\rangle$ has a projection of length less than $\epsilon^{1 / 4}$ onto $W^{\prime}$, much less than one. In terms of probabilities, the probability of measuring $W^{\prime}$ in the state $|\rho\rangle$ is less than $\sqrt{\epsilon}$. The classical union bound on probabilities would give $\epsilon$.

Let us define the 'intersection' of subspaces to be the complement of the tilted span of the complementary subspaces. Applying this recipe to projectors $\Pi^{X}, \Pi^{Y}, \Pi^{X, Y}$ achieving the maxima in the definitions of the three entropic quantities $I_{H}^{\epsilon}(X: Y Z), I_{H}^{\epsilon}(Y: X Z), I_{H}^{\epsilon}(X Y: Z)$ gives us a projector 
$\Pi$ that satisfies Property 1 with lower bound $1-O(\sqrt{\epsilon})$ by setting $\alpha=\sqrt{\epsilon}$ in the upper bound of Proposition 2 However, it is not clear whether $\Pi$ satisfies the Properties 2, 3, 4 even approximately. This is because in order to prove, say, Property 2, one has to use the lower bound of Proposition 2 with $\alpha=\sqrt{\epsilon}$, which would give an upper bound of at least $\sqrt{\epsilon}$ for Property 2 !

Hence we need another new idea in order to obtain a robust notion of intersection of subspaces. The new idea comes from a phenomenon that we will call smoothing and augmentation. Let us see a simple example of this. Consider a pure state $\psi^{X Y}$ on the bipartite Hilbert space $\mathcal{X} \otimes \mathcal{Y}$. Expressed in the computational basis, the state looks like $|\psi\rangle^{X Y}=\sum_{x y} s_{x y}|x\rangle^{X} \otimes|y\rangle^{Y}$. Suppose $|\psi\rangle^{X Y}$ is isometrically tilted in the following fashion to make a state $\left|\psi^{\prime}\right\rangle^{X^{\prime} Y^{\prime}}$ in a larger Hilbert space $\mathcal{X}^{\prime} \otimes \mathcal{Y}^{\prime}$ as follows:

$$
\begin{array}{r}
\left|\psi^{\prime}\right\rangle^{X^{\prime} Y^{\prime}} \\
:=\sum_{x y} s_{x y}\left(\sqrt{1-2 \epsilon}|x\rangle^{X}+\sqrt{\epsilon}|x\rangle^{X_{x}}+\sqrt{\epsilon}|x\rangle^{X_{y}}\right) \\
\otimes\left(\sqrt{1-2 \epsilon}|y\rangle^{Y}+\sqrt{\epsilon}|y\rangle^{Y_{x}}+\sqrt{\epsilon}|y\rangle^{Y_{y}}\right),
\end{array}
$$

where

$$
\begin{aligned}
& x^{\prime}:=x \oplus \bigoplus x_{x} \oplus \bigoplus x_{y}, \\
& y^{\prime}:=y \oplus \bigoplus y_{x} \oplus \bigoplus y_{y},
\end{aligned}
$$

$\mathcal{X}_{x}, \mathcal{X}_{y}$ are orthogonal Hilbert spaces of the same dimension as $X$ indexed by the computational basis states $|x\rangle^{X},|y\rangle^{Y}$ and $\mathcal{Y}_{x}, \boldsymbol{y}_{y}$ are orthogonal Hilbert spaces of the same dimension as $\mathcal{Y}$ indexed by the computational basis states $|x\rangle^{X},|y\rangle^{Y}$. Then,

$$
\begin{aligned}
& \left(\psi^{\prime}\right)^{X^{\prime}} \\
& =\operatorname{Tr}_{Y^{\prime}}\left[\sum_{x x^{\prime}} \sum_{y y^{\prime}} s_{x y} s_{x^{\prime} y^{\prime}}^{*}\left(\sqrt{1-2 \epsilon}|x\rangle^{X}+\sqrt{\epsilon}|x\rangle^{X_{x}}+\sqrt{\epsilon}|x\rangle^{X_{y}}\right)\right. \\
& \left(\sqrt { 1 - 2 \epsilon } \left\langle\left.x^{\prime}\right|^{X}+\sqrt{\epsilon}\left\langle\left. x^{\prime}\right|^{X_{x^{\prime}}}+\sqrt{\epsilon}\left\langle\left. x^{\prime}\right|^{X_{y^{\prime}}}\right)\right.\right.\right. \\
& \otimes\left(\sqrt{1-2 \epsilon}|y\rangle^{Y}+\sqrt{\epsilon}|y\rangle^{Y_{x}}+\sqrt{\epsilon}|y\rangle^{Y_{y}}\right) \\
& \left(\sqrt { 1 - 2 \epsilon } \left\langle\left.y^{\prime}\right|^{Y}+\sqrt{\epsilon}\left\langle\left. y^{\prime}\right|^{Y_{x^{\prime}}}+\sqrt{\epsilon}\left\langle\left. y^{\prime}\right|^{Y_{y^{\prime}}}\right)\right.\right.\right. \\
& =\sum_{x x^{\prime}} \sum_{y} s_{x y} s_{x^{\prime} y}^{*}\left(\sqrt{1-2 \epsilon}|x\rangle^{X}+\sqrt{\epsilon}|x\rangle^{X_{x}}+\sqrt{\epsilon}|x\rangle^{X_{y}}\right) \\
& =\left(\psi^{\prime \prime}\right)^{X^{\prime}}+\phi^{X^{\prime}}, \quad
\end{aligned}
$$

where

$$
\begin{array}{r}
\left(\psi^{\prime \prime}\right)^{X^{\prime}} \\
:=\sum_{x x^{\prime}}\left(\sum_{y} s_{x y} s_{x^{\prime} y}^{*}\right)\left(\sqrt{1-2 \epsilon}|x\rangle^{X}+\sqrt{\epsilon}|x\rangle^{X_{x}}\right) \\
\left(\sqrt { 1 - 2 \epsilon } \left\langle\left.x^{\prime}\right|^{X}+\sqrt{\epsilon}\left\langle\left. x^{\prime}\right|^{X_{x}}\right)\right.\right.
\end{array}
$$

is an isometric tilt of $\psi^{X}$ and

$\phi^{X^{\prime}}$

$$
:=\sum_{x x^{\prime}} \sum_{y} s_{x y} s_{x^{\prime} y}^{*}\left(( \sqrt { 1 - 2 \epsilon } | x \rangle ^ { X } + \sqrt { \epsilon } | x \rangle ^ { X _ { x } } ) \left(\sqrt{\epsilon}\left\langle\left. x^{\prime}\right|^{X_{y}}\right)\right.\right.
$$

$$
\begin{aligned}
& +\left(\sqrt{\epsilon}|x\rangle^{X_{y}}\right)\left(\sqrt { 1 - 2 \epsilon } \left\langle\left.x^{\prime}\right|^{X}+\sqrt{\epsilon}\left\langle\left. x^{\prime}\right|^{X_{x}}\right)\right.\right. \\
& \left.+\epsilon|x\rangle^{X_{y}}\left\langle x^{\prime}\right|\right)
\end{aligned}
$$

is a Hermitian matrix. Observe that the Hilbert spaces $\mathcal{X}_{y}$, as $|y\rangle$ runs over the computational basis of $\mathcal{Y}$, are orthogonal. Let $\left|\psi_{y}\right\rangle^{X}:=\sum_{x} s_{x y}|x\rangle^{X}$ be the unnormalised state that results when $Y$ is measured in the computational basis and the outcome is $y$. Define the following scaled isometric embeddings from $X$ into $X^{\prime}$ :

$$
\begin{aligned}
\mathcal{T}_{X}\left(|x\rangle^{X}\right) & :=\sqrt{1-2 \epsilon}|x\rangle^{X}+\sqrt{\epsilon}|x\rangle^{X_{x}}, \\
\mathcal{T}_{y}\left(|x\rangle^{X}\right) & :=|x\rangle^{X_{y}}
\end{aligned}
$$

It is now easy to see that

$$
\begin{aligned}
& \left\|\phi^{X^{\prime}}\right\|_{\infty} \\
& \leq \| \sqrt{\epsilon} \sum_{y} \mathcal{T}_{X}\left|\psi_{y}\right\rangle^{X}\left\langle\psi_{y}\left|\mathcal{T}_{y}^{\dagger}\left\|_{\infty}+\right\| \sqrt{\epsilon} \sum_{y} \mathcal{T}_{y}\right| \psi_{y}\right\rangle^{X}\left\langle\psi_{y}\right| \mathcal{T}_{X}^{\dagger} \|_{\infty} \\
& \quad+\epsilon \| \sum_{y} \mathcal{T}_{y}\left|\psi_{y}\right\rangle^{X}\left\langle\psi_{y}\right| \mathcal{T}_{y}^{\dagger} \|_{\infty} \\
& \leq \quad 2 \sqrt{\epsilon|\mathcal{Y}|}\left(\max _{y} \| \mathcal{T}_{X}\left|\psi_{y}\right\rangle^{X}\left\langle\psi_{y}\right| \|_{\infty}\right)+\epsilon\left(\max _{y} \|\left|\psi_{y}\right\rangle^{X}\left\langle\psi_{y}\right| \|_{\infty}\right) \\
& =2 \sqrt{\epsilon|\mathcal{Y}|}\left(\max _{y} \|\left|\psi_{y}\right\rangle^{X} \|_{2}^{2}\right)+\epsilon\left(\max _{y} \|\left|\psi_{y}\right\rangle^{X} \|_{2}^{2}\right) \\
& \leq \quad 3 \sqrt{\epsilon|\mathcal{Y}|}\left(\max _{y} \|\left|\psi_{y}\right\rangle^{X} \|_{2}^{2}\right) .
\end{aligned}
$$

Recall that $\|\left|\psi_{y}\right\rangle^{X} \|_{2}^{2}$ is the probability of obtaining the outcome $y$. Thus, if the probability distribution of the outcomes obtained by measuring $Y$ has low $\ell_{\infty}$-norm or in other words is 'smooth', then $\left\|\left(\psi^{\prime}\right)^{X^{\prime}}-\left(\psi^{\prime \prime}\right)^{X^{\prime}}\right\|_{\infty}$ is small. The import of this statement is that though $\left(\psi^{\prime}\right)^{X^{\prime} Y^{\prime}}$ is tilted along both ' $\mathrm{X}$ ' and ' $y$ ' directions, $\left(\psi^{\prime}\right)^{X^{\prime}}$ is primarily tilted along ' $\mathrm{x}$ ' direction only since it is close to $\left(\psi^{\prime \prime}\right)^{X^{\prime}}$. This desired property is what we mean by smoothing $\operatorname{Tr}_{Y^{\prime}}[\cdot]$.

Note however that smoothing $\operatorname{Tr}_{Y^{\prime}}[\cdot]$ in the above example is possible only if the probability distribution obtained by measuring $Y$ is 'smooth'. This may not be the case for an arbitrary state $\psi^{X Y}$. So we augment $\psi^{X Y}$ to a state $\hat{\psi}^{\hat{X} \hat{Y}}$ defined on the Hilbert space $\hat{X} \otimes \hat{Y}$ as follows:

$$
\hat{\psi}^{\hat{X} \hat{Y}}:=|\mathcal{L}|^{-2} \psi^{X Y} \otimes \mathbb{1}^{L_{X}} \otimes \mathbb{1}^{L_{Y}},
$$

where $\mathcal{L}_{X}, \mathcal{L}_{Y}$ are new Hilbert spaces of the same dimension $|\mathcal{L}|, \hat{\mathcal{X}}:=\mathcal{X} \otimes \mathcal{L}_{X}, \hat{\mathcal{Y}}:=\mathcal{Y} \otimes \mathcal{L}_{Y}$. We can now tilt $\hat{\psi}^{\hat{X} \hat{Y}}$ to a state $\left(\hat{\psi}^{\prime}\right)^{\prime} \hat{X}^{\prime}$ in the same fashion as above, where tilting a mixed state means tilting its constituent pure states. If the dimension $|\mathcal{L}|$ of the augmenting system is large enough, the operation of $\operatorname{Tr}_{Y^{\prime}}[\cdot]$ indeed achieves smoothing in the sense described above since

$$
\left\|\left(\hat{\psi}^{\prime}\right)^{\hat{X}^{\prime}}-\left(\hat{\psi}^{\prime \prime}\right)^{\hat{X}^{\prime}}\right\|_{\infty} \leq 3 \sqrt{\frac{\epsilon|\mathcal{Y}|}{|\mathcal{L}|}} .
$$

We refer to this entire process as smoothing and augmentation. 
The above paragraph can be summarised in the observation that though partial trace can increase the $\ell_{\infty}$-norm of a quantum state in general, the increase is negative or only slightly positive if the state is highly correlated between the traced out part and the untraced out part. As we will see later on, in general after augmentation it is possible to tilt a multipartite quantum state, say $\rho^{X_{1} \cdots X_{k}}$, along several directions with carefully chosen amplitudes so as to get a state $\left(\rho^{\prime}\right)^{X_{1} \cdots X_{k}}$ with the property it is highly correlated across all partitions of the systems $X_{1}, \ldots, X_{k}$. As a consequence, for example, for any subset \{\}$\neq S \subset[k],\left(\rho^{\prime}\right)^{X_{S}} \otimes\left(\rho^{\prime}\right)^{X_{\bar{S}}}$ is tilted along $(S, \bar{S})$ for all practical purposes even though $\left(\rho^{\prime}\right)^{X_{[k]}}:=\left(\rho^{\prime}\right)^{X_{1} \cdots X_{k}}$ is tilted along $[k]$. The formal statement and proof of this result, for which we have to define a more nuanced tilting operation called matrix tilt, is technically intricate and can be found in Proposition 4 below.

The idea of tilting along both ' $x$ ' and ' $y$ ' directions combined with smoothing and augmentation can be used as a starting point and further refined, leading finally to a proof of the desired inner bound (Equation 3 for the cq-MAC. We do so with the following sequence of steps. A full proof is given in Section 6 below.

1. Enlarge the Hilbert space $\mathcal{X} \otimes \mathcal{Y} \otimes \mathcal{Z}$ suitably and consider a 'perturbed' version $C^{\prime}$ of the channel $C$. The channel $C^{\prime}$ maps an input pair $(x, y)$ to a state $\rho_{x, y}^{\prime}$ close to $\rho_{x, y}$. This gives a state $\left(\rho^{\prime}\right)^{X Y Z}$ close to $\rho^{X Y Z}$. A codebook achieving a certain rate point for channel $C^{\prime}$ with a certain error achieves the same rate point for channel $C$ with only slightly more error. The state $\rho_{x, y}^{\prime}$ is obtained by augmenting $\rho_{x, y}$ and then tilting it along ' $\mathrm{x}$ ' and ' $y$ ' directions;

2. Due to smoothing and augmentation described above, $\left(\rho^{\prime}\right)^{X Z} \otimes\left(\rho^{\prime}\right)^{Y}$ and $\left(\rho^{\prime}\right)^{Y Z} \otimes\left(\rho^{\prime}\right)^{X}$ are extremely close to the states $\rho^{X Z} \otimes \rho^{Y}$ and $\rho^{Y Z} \otimes \rho^{X}$ tilted only along ' $\mathrm{x}$ ' and only along ' $y$ ' respectively. The state $\left(\rho^{\prime}\right)^{X} \otimes\left(\rho^{\prime}\right)^{Y} \otimes$ $\left(\rho^{\prime}\right)^{Z}$ is extremely close to the untilted state $\rho^{X} \otimes \rho^{Y} \otimes \rho^{Z}$;

3. We now define the 'intersection' POVM element $\Pi$ to be the projector onto the complement of the tilted span of the complements of the supports of $\Pi^{X}, \Pi^{Y}$, $\Pi^{X, Y}$. Using Proposition 2, we can show that Property 1 holds with lower bound $1-O(\epsilon)$ under the projector $\Pi$;

4. Finally, we notice from the construction of $\Pi$ that Properties 2, 3, 4 are easily satisfied by $\Pi$ for $\rho^{X Z} \otimes \rho^{Y}$ tilted only along ' $\mathrm{x}$ ', $\rho^{Y Z} \otimes \rho^{X}$ tilted only along 'y' and untilted $\rho^{X} \otimes \rho^{Y} \otimes \rho^{Z}$, with upper bounds exactly the same as in the ideal case. Since the states $\left(\rho^{\prime}\right)^{X Z} \otimes\left(\rho^{\prime}\right)^{Y}$, $\left(\rho^{\prime}\right)^{Y Z} \otimes\left(\rho^{\prime}\right)^{X},\left(\rho^{\prime}\right)^{X} \otimes\left(\rho^{\prime}\right)^{Y} \otimes\left(\rho^{\prime}\right)^{Z}$ are extremely close to the tilted versions of $\rho^{X Z} \otimes \rho^{Y}, \rho^{Y Z} \otimes \rho^{X}, \rho^{X} \otimes \rho^{Y} \otimes \rho^{Z}$ described above, we finally conclude that they satisfy Properties 2, 3, 4 under $\Pi$ with upper bounds almost as good as in the ideal case;

5. Thus, $\Pi$ can be used by Charlie in order to construct a decoding algorithm for the original channel $C$ that achieves any rate pair in the region described in Equation 3 with average error probablity at most $O(\epsilon)$.

The strategy outlined above enables us to prove the following one-shot quantum joint typicality lemma.

Let $A_{1} \ldots A_{k}$ be a k-partite quantum system with each $A_{i}$ isomorphic to a Hilbert space $\mathcal{H}$. Let $\rho^{A_{1} \ldots A_{k}}$ be a quantum state in $A_{1} \ldots A_{k}$. For a subset $S \subseteq[k]$, let $A_{S}$ denote the systems $\left\{A_{s}\right.$ : $s \in S\}$. Let $\rho^{A_{S}}$ denote the marginal state on $A_{S}$ obtained by tracing out the systems in $\bar{S}:=$ $[k] \backslash S$ from $\rho^{A_{1} \ldots A_{k}}$. Let $0<\epsilon<1$. Let $\mathcal{K}$ be $a$ Hilbert space of dimension

$$
\frac{2^{13(k+1)}(2|\mathcal{H}|)^{6 k(k+1)}}{(1-\epsilon)^{6(k+1)}} .
$$

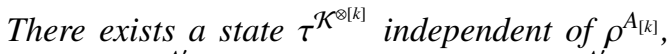
a state $\left(\rho^{\prime}\right)^{A_{[k]}^{\prime}}$, and a POVM element $\Pi^{\prime A_{[k]}^{\prime}}$ on $A_{1}^{\prime} \ldots A_{k}^{\prime}$ where $A_{i}^{\prime} \cong A_{i} \otimes \mathcal{K}$, with the following properties:

$$
\begin{aligned}
& \text { 1. }\left\|\left(\rho^{\prime}\right)^{A_{[k]}^{\prime}}-\rho^{A_{[k]}} \otimes \tau^{\mathcal{K}^{\otimes[k]}}\right\|_{1} \leq 2^{\frac{k}{2}+1} \epsilon^{\frac{1}{4 k}} ; \\
& \text { 2. } \operatorname{Tr}\left[\left(\Pi^{\prime}\right)^{A_{[k]}^{\prime}}\left(\rho^{\prime}\right)^{A_{[k]}^{\prime}}\right] \geq 1-2^{8(k+1)^{k}} \epsilon^{\frac{1}{4}}-2^{\frac{k}{2}+1} \epsilon^{\frac{1}{4 k}} ; \\
& \text { 3. For every set } S,\{\} \neq S \subset[k],
\end{aligned}
$$

$$
\operatorname{Tr}\left[\left(\Pi^{\prime}\right)^{A_{[k]}^{\prime}}\left(\left(\rho^{\prime}\right)^{A_{S}^{\prime}} \otimes\left(\rho^{\prime}\right)^{A_{\bar{S}}^{\prime}}\right)\right] \leq 2^{-D_{H}^{\epsilon}\left(\rho^{A}[k] \| \rho^{A} S \otimes \rho^{A_{\bar{S}}}\right) .}
$$

\subsection{Related work}

The bottleneck of simultaneous decoding was first pointed out by Fawzi et al [17] in their paper on the classical quantum interference channel. The authors obtained a simultaneous decoder for the two sender cq-MAC in the asymptotic iid setting. At around the same time, $\mathrm{Xu}$ and Wilde [18] obtained a simultaneous decoder for sending classical information over a two sender entanglement assisted quantum MAC in the asymptotic iid setting. Subsequently, Sen [10] obtained simultaneous decoders for two sender and a restricted type of three sender cq-MACs in the asymptotic iid setting. The simultaneous decoder for the restricted three sender cqMAC sufficed to obtain the so-called Chong-Motani-GargEl Gamal inner bound for the classical quantum interference channel in the asymptotic iid setting, matching the best classical result for this channel. None of these constructions extended to general three sender MACs, nor to the asymptotic non-iid or the one shot settings. Simultaneous decoders have been recently used in several papers e.g. [19], but the inner bounds obtained there are suboptimal compared to known inner bounds when restricted to the asymptotic iid setting. In a companion paper [11], we apply our quantum joint typicality lemma to obtain one shot simultaneous decoders for several multiterminal classical quantum channels like broadcast and interference channels, matching the best inner bounds known for these channels in the classical asymptotic iid setting. 
On a different note Dutil [20] pointed out a related bottleneck of 'simultaneous smoothing', which was further discussed by Drescher and Fawzi [21]. The aim of simultaneous smoothing is to replace a multipartite quantum state, e.g. $\rho^{A B}$, by another 'perturbed' state $\left(\rho^{\prime}\right)^{A B}$ which is $\delta \equiv \delta(\epsilon)$ close to it in trace distance such that

$$
\begin{aligned}
H_{\min }(A)_{\rho^{\prime}} & \leq c(\delta) H_{\min }^{\epsilon}(A)_{\rho^{\prime}}, \\
H_{\min }(B)_{\rho^{\prime}} & \leq c(\delta) H_{\min }^{\epsilon}(B)_{\rho^{\prime}}, \\
H_{\min }(A B)_{\rho^{\prime}} & \leq c(\delta) H_{\min }^{\epsilon}(A B)_{\rho^{\prime}},
\end{aligned}
$$

where $\delta(\epsilon)$ is a dimension independent function of $\epsilon, c(\delta)$ is a dimension independent function of $\delta, H_{\min }(\rho):=-\log \|\rho\|_{\infty}$, $H_{\text {min }}^{\epsilon}(\rho):=\max _{\rho^{\prime}:\left\|\rho^{\prime}-\rho\right\|_{1} \leq \delta} H_{\min }\left(\rho^{\prime}\right)$. The main difference between simultaneous smoothing and our notion of joint typicality is that joint typicality requires the existence of a single perturbed state as well as a single POVM satisfying certain properties, whereas there is no corresponding notion of POVM for simultaneous smoothing. Simultaneous smoothing seems more relevant for so-called 'covering type' problems whereas joint typicality seems more relevant for socalled 'packing type' problems in Shannon theory.

Our joint typicality lemma can be viewed through the lens of quantum hypothesis testing as a problem where there is one positive and a set of negative hypotheses, each negative hypothesis begin a convex combination of tensor products of marginals of the positive hypothesis. Related work has been done by Berta, Brandão and Hirche [22]. The main difference between their work and ours is that they work in the asymptotic iid setting and try to find a single POVM that minimises the largest probability of accepting a negative hypothesis from the negative hypothesis set while accepting all positive hypotheses from the positive hypothesis set with probability close to one. On the other hand, we work in the one shot setting and find a single POVM that accepts the positive hypothesis with probability close to one and rejects all the negative hypotheses with probabality at least as much as the optimal POVM for each individual negative hypothesis. Viewed in this fashion, our result may seem to be more general. But actually the two results are incomparable because the negative hypotheses in our result are of the form of tensor products of marginals of a state close to the positive hypothesis state, wheares the negative hypotheses in Berta et al are of the form of convex combinations of iid states from any a priori given set.

\subsection{Organisation of the paper}

In the next section, we state some preliminary facts which will be useful throughout the paper. Section 3 defines and proves the union properties of the tilted span and $A$-tilted span of subspaces. Section 4 gives a self-contained proof of a simplified one shot inner bound for the quantum multiple access channel with two senders. In Section 5 we prove the socalled one-shot quantum joint typicality lemma, which manages to construct a robust notion of intersection of POVM elements achieving a given set of hypothesis testing mutual information quantitites. The essential part of the proof of the one-shot quantum joint typicality lemma is encapsulated into a proposition which is proved in Appendix I. We formally prove the achievability of the rate region described by Equation 3 in Section 6. Finally, we make some concluding remarks and list some open problems in Section 7 .

\section{Preliminaries}

All Hilbert spaces in this paper are finite dimensional. The symbol $\oplus$ always denotes the orthogonal direct sum of Hilbert spaces. For a subspace $X$ of a Hilbert space $\mathcal{H}$, let $\Pi_{X}^{\mathcal{H}}$ denote the orthogonal projection in $\mathcal{H}$ onto $X$. When clear from the context, we may use $\Pi_{X}$ instead of $\Pi_{X}^{\mathcal{H}}$ for brevity of notation.

By a quantum state or a density matrix in a Hilbert space $\mathcal{H}$, we mean a Hermitian, positive semidefinite linear operator on $\mathcal{H}$ with trace equal to one. By a POVM element $\Pi$ in $\mathcal{H}$, we mean a Hermitian positive semidefinite linear operator on $\mathcal{H}$ with eigenvalues between 0 and 1 . Stated in terms of inequalities on Hermitian operators, $\mathbb{0} \leq \Pi \leq \mathbb{1}$, where $\mathbb{0}, \mathbf{1}$ denote the zero and identity operators on $\mathcal{H}$. In what follows, we shall use several times the Gelfand-Naimark theorem which is stated below for completeness.

Fact 2 (Gelfand-Naimark) Let $\Pi$ be a POVM element in a Hilbert space $\mathcal{H}$. For any integer $d \geq 2$, there exists an orthogonal projection $\Pi^{\prime}$ in $\mathcal{H} \otimes \mathbb{C}^{d}$ such that

$$
\operatorname{Tr}[\Pi A]=\operatorname{Tr}\left[\Pi^{\prime}\left(A \otimes|0\rangle\left\langle\left. 0\right|^{\mathbb{C}^{d}}\right)\right]\right.
$$

for all linear operators A acting on $\mathcal{H}$. Above, $|0\rangle$ is a fixed vector, independent of $\Pi$ and $A$, in $\mathbb{C}^{d}$.

Since quantum probability is a generalisation of classical probability, one can talk of a so-called 'classical POVM element', as done in the introduction. Suppose we have a probability distribution $p(x), x \in \mathcal{X}$. A classical POVM element on $\mathcal{X}$ is a function $f: \mathcal{X} \rightarrow[0,1]$. The probability of accepting the POVM element $f$ is then $\sum_{x: x \in \mathcal{X}} p(x) f(x)$. One can continue to use the operator formalism for classical probablity with the understanding that density matrices and POVM elements are now diagonal matrices.

Let $\|v\|_{2}$ denote the $\ell_{2}$-norm of a vector $v \in \mathcal{H}$. For an operator $A$ on $\mathcal{H}$, we use $\|A\|_{1}$ to denote the Schatten $\ell_{1}$ norm, also known as trace norm, of $A$, which is nothing but the sum of singular values of $A$. We use $\|A\|_{\infty}$ to denote the Schatten $\ell_{\infty}$-norm, also known as operator norm, of $A$, which is nothing but the largest singular value of $A$. For operators $A, B$ on $\mathcal{H}$, we have the inequality

$$
|\operatorname{Tr}[A B]| \leq\|A B\|_{1} \leq \min \left\{\|A\|_{1}\|B\|_{\infty},\|A\|_{\infty}\|B\|_{1}\right\} .
$$

Let $\mathcal{X}$ be a finite set. By a classical-quantum state on $\mathcal{X H}$ we mean a quantum state of the form $\rho^{\mathcal{X H}}=\sum_{x \in \mathcal{X}} p_{x}|x\rangle\left\langle\left. x\right|^{\mathcal{X}} \otimes\right.$ $\rho_{x}^{\mathcal{H}}$, where $x$ ranges over computational basis vectors of $\mathcal{X}$ viewed as a Hilbert space, $\left\{p_{x}\right\}_{x \in \mathcal{X}}$ is a probability distribution on $\mathcal{X}$ and the operators $\rho_{x}, x \in \mathcal{X}$ are quantum states in $\mathcal{H}$. We will also use the terminology that $\rho$ is classical on $\mathcal{X}$ and quantum on $\mathcal{H}$. 
For a positive integer $k$, we use $[k]$ to denote the set $\{1,2, \ldots, k\}$. If $k=0$, we define $[k]:=\{\}$. Let $c$ be a nonnegative integer and $k$ a positive integer. We shall study systems that are classical on $\mathcal{X}^{\otimes[c]}$ and quantum on $\mathcal{H}^{\otimes[k]}$, where $[c]$ and $[k]$ are treated as disjoint sets. We will use the notation $[c] \cup[k]$ to denote the union $[c] \cup[k]$ keeping in mind that $[c],[k]$ are disjoint. A subpartition $\left(S_{1}, \ldots, S_{l}\right)$ of $[k]$, denoted by $\left(S_{1}, \ldots, S_{l}\right) \vdash[k]$, is a collection of non-empty, pairwise disjoint subsets of $[k]$. Note that the order of subsets does not matter in defining a subpartition $\left(S_{1}, \ldots, S_{l}\right)$. For a subset $T \subseteq[c] \cup[k]$ satisfying $T \cap[k] \neq\{\}$, we use the notation $\left(S_{1}, \ldots, S_{l}\right)+\vdash T$ to denote a so-called pseudosubpartition of $T$ intersecting $[k]$ non-trivially i.e. for $i \in[l]$, $S_{i} \subseteq T, S_{i} \cap[k] \neq\{\}$ and for $i \neq j \in[l], S_{i} \cap S_{j} \cap[k]=\{\}$. There is a natural partial order called the refinement partial order on the pseudosubpartitions of $T$ intersecting [k] nontrivially, where $\left(S_{1}, \ldots, S_{l}\right) \leq\left(R_{1}, \ldots, R_{m}\right)$ if there is a function $f:[l] \rightarrow[m]$ such that $S_{i} \subseteq R_{f(i)}$ for all $i \in[l]$. Under this partial order, the pseudosubpartitions form a lattice with minimum element being the empty pseudosubpartition with $l=0$, and maximum element being the full block with $l=1$ and $S_{1}=T$. It is easy to see that the number of pseudosubpartitions of $T$ is at most $2^{|T \cap[k]||T \cap[c]|}(|T \cap[k]|+1)^{|T \cap[k]|}$, and the number of pseudosubpartitions of $T$ with $l$ blocks is at most $\frac{2^{l|T \cap[c]|}(l+1)^{|T \cap[k]|}}{l !}$.

Suppose we have a $k$-partite quantum system $A_{1} \cdots A_{k}$. For a non-empty set $S \subseteq[k]$, let $A_{S}$ denote the systems $\left\{A_{s}: s \in S\right\}$. Similarly, if $\rho$ is a quantum state in $A_{[k]}, \rho^{A_{S}}$ will denote its marginal in $A_{S}$. Thus, $\rho \equiv \rho^{A_{[k]}}$. If $\mathbf{x}$ is a computational basis vector of $\mathcal{X}^{\otimes[c]}$, for a subset $S \subseteq[c], \mathbf{x}_{S}$ will denote its restriction to the system $\mathcal{X}^{\otimes S}$. Thus, $\mathbf{x} \equiv \mathbf{x}_{[c]}$. We also use $\mathbf{x}_{S}$ to denote computational basis vectors of $\mathcal{X}^{\otimes S}$ without reference to the systems in $[c] \backslash S$. For a subset $S \subseteq[c] \cup[k]$ and a computational basis vector $\mathbf{l}$ of $\mathcal{L}^{\otimes[c] \cup[k]}$, the notation $\mathbf{l}_{S}$ has the analogous meaning. The notation $(\cdot)^{\otimes S}$ denotes a tensor product only for the coordinates in $S$.

We recall the definition of the hypothesis testing relative entropy as given by Wang and Renner [8]. Very similar quantities were defined and used in earlier works [23, 24].

Definition 1 Let $\alpha, \beta$ be two quantum states in the same Hilbert space. Let $0 \leq \epsilon<1$. Then the hypothesis testing relative entropy of $\alpha$ with respect to $\beta$ is defined by

$$
D_{H}^{\epsilon}(\alpha \| \beta):=\max _{\Pi: \operatorname{Tr}[\Pi \alpha] \geq 1-\epsilon}-\log \operatorname{Tr}[\Pi \beta],
$$

where the maximisation is over all POVM elements $\Pi$ acting on the Hilbert space.

The definition quantifies the minimum probability of 'accepting' $\beta$ by a POVM element $\Pi$ that 'accepts' $\alpha$ with probability at least $1-\epsilon$. From the definition, it is easy to see that if $\epsilon<\epsilon^{\prime}, D_{H}^{\epsilon}(\alpha \| \beta)<D_{H}^{\epsilon^{\prime}}(\alpha \| \beta)$. We will require the following property of the so-called hypothesis testing mutual information.

Proposition 1 Let $0 \leq \epsilon<1$. Let $\rho^{A B}$ be a quantum state in a bipartite system $A B$. Define the hypothesis testing mutual information $I_{H}^{\epsilon}(A: B)_{\rho}:=D_{H}^{\epsilon}\left(\rho^{A B} \| \rho^{A} \otimes \rho^{B}\right)$. Then,

$$
I_{H}^{\epsilon}(A: B)_{\rho} \leq 2 \log \min \{|A|,|B|\}+3 \log \frac{1}{1-\epsilon}+6 \log 3-4 .
$$

Proof: Let $C$ be a third system and $\rho^{A B C}$ a purification of $\rho^{A B}$. Let $\Pi^{A B}$ be the optimising POVM element for $D_{H}^{\epsilon}\left(\rho^{A B} \| \rho^{A} \otimes\right.$ $\left.\rho^{B}\right)$. Define $\Pi^{A B C}:=\Pi^{A B} \otimes \mathbb{1}^{C}$. Let $|A| \leq|B|$ without loss of generality. We will show that

$$
\operatorname{Tr}\left[\Pi^{A B C}\left(\rho^{A} \otimes \rho^{B C}\right)\right] \geq \frac{2^{4}(1-\epsilon)^{3}}{3^{6}|A|^{2}}
$$

which would prove the proposition.

Let $|\rho\rangle^{A B C}$ denote the pure state $\rho^{A B C}$ in ket vector form. Let

$$
|\rho\rangle^{A B C}=\sum_{a=1}^{|A|} \sqrt{p_{a}}\left|x_{a}\right\rangle^{A} \otimes\left|y_{a}\right\rangle^{B C}
$$

be a Schmidt decomposition for the bipartition $(A, B C)$, where $\sum_{a=1}^{|A|} p_{a}=1$. Fix $0<\delta<1$. Define

$$
\hat{A}:=\left\{a \in[|A|]: p_{a} \geq \frac{\delta(1-\epsilon)}{|A|}\right\} .
$$

Using the triangle inequality, we get

$$
\begin{aligned}
\sqrt{1-\epsilon} \leq & \| \Pi^{A B C}|\rho\rangle^{A B C} \|_{2} \\
\leq & \| \Pi^{A B C}\left(\sum_{a \in \hat{A}} \sqrt{p_{a}}\left|x_{a}\right\rangle^{A} \otimes\left|y_{a}\right\rangle^{B C}\right) \|_{2} \\
& +\| \Pi^{A B C}\left(\sum_{a \notin \hat{A}} \sqrt{p_{a}}\left|x_{a}\right\rangle^{A} \otimes\left|y_{a}\right\rangle^{B C}\right) \|_{2} \\
\leq & \sum_{a \in \hat{A}} \sqrt{p_{a}} \| \Pi^{A B C}\left(\left|x_{a}\right\rangle^{A} \otimes\left|y_{a}\right\rangle^{B C}\right) \|_{2} \\
& +\| \sum_{a \notin \hat{A}} \sqrt{p_{a}}\left|x_{a}\right\rangle^{A} \otimes\left|y_{a}\right\rangle^{B C} \|_{2} \\
= & \sum_{a \in \hat{A}} \sqrt{p_{a}} \| \Pi^{A B C}\left(\left|x_{a}\right\rangle^{A} \otimes\left|y_{a}\right\rangle^{B C}\right) \|_{2}+\sqrt{\sum_{a \notin \hat{A}} p_{a}} \\
< & \sum_{a \in \hat{A}} \sqrt{p_{a}} \| \Pi^{A B C}\left(\left|x_{a}\right\rangle^{A} \otimes\left|y_{a}\right\rangle^{B C}\right) \|_{2}+\sqrt{\delta(1-\epsilon)},
\end{aligned}
$$

which implies that

$$
\sum_{a \in \hat{A}} \sqrt{p_{a}} \| \Pi^{A B C}\left(\left|x_{a}\right\rangle^{A} \otimes\left|y_{a}\right\rangle^{B C}\right) \|_{2}>\sqrt{1-\epsilon}(1-\sqrt{\delta}) .
$$

Using the Cauchy-Schwarz inequality, we get

$$
\sqrt{1-\epsilon}(1-\sqrt{\delta})<\sqrt{\sum_{a \in \hat{A}} p_{a}} \sqrt{\sum_{a \in \hat{A}} \| \Pi^{A B C}\left(\left|x_{a}\right\rangle^{A} \otimes\left|y_{a}\right\rangle^{B C}\right) \|_{2}^{2}}
$$

which implies that

$$
\sqrt{\sum_{a \in \hat{A}} \| \Pi^{A B C}\left(\left|x_{a}\right\rangle^{A} \otimes\left|y_{a}\right\rangle^{B C}\right) \|_{2}^{2}}>\sqrt{1-\epsilon}(1-\sqrt{\delta}) .
$$


Now,

$$
\begin{aligned}
\operatorname{Tr} & {\left[\Pi^{A B C}\left(\rho^{A} \otimes \rho^{B C}\right)\right] } \\
& =\operatorname{Tr}\left[\Pi^{A B C}\left(\sum_{a=1}^{|A|} p_{a}\left|x_{a}\right\rangle\left\langle x_{a}\right|\right)^{A} \otimes\left(\sum_{a^{\prime}=1}^{|A|} p_{a^{\prime}}\left|y_{a^{\prime}}\right\rangle\left\langle y_{a^{\prime}}\right|\right)^{B C}\right] \\
& \geq \sum_{a \in \hat{A}} p_{a}^{2} \operatorname{Tr}\left[\Pi^{A B C}\left(\left|x_{a}\right\rangle\left\langle\left. x_{a}\right|^{A} \otimes \mid y_{a}\right\rangle\left\langle\left. y_{a}\right|^{B C}\right)\right]\right. \\
& =\sum_{a \in \hat{A}} p_{a}^{2} \| \Pi^{A B C}\left(\left|x_{a}\right\rangle^{A} \otimes\left|y_{a}\right\rangle^{B C}\right) \|_{2}^{2} \\
& \geq \frac{\delta^{2}(1-\sqrt{\delta})^{2}(1-\epsilon)^{3}}{|A|^{2}} .
\end{aligned}
$$

Taking $\delta=4 / 9$ gives the largest lower bound above, and completes the proof of the proposition.

We will also need the so-called non-commutative union bound first proved by Sen [10], and later improved by Gao [15] and Oskouei et al [16].

Fact 3 (Noncommutative union bound) Let $\rho$ be a positive semidefinite operator on a Hilbert space $\mathcal{H}$ and $\operatorname{Tr} \rho \leq 1$. Let $\Pi_{1}^{\prime}, \ldots, \Pi_{k}^{\prime}$ be orthogonal projectors in $\mathcal{H}$. Define $\Pi_{i}:=$ $1-\Pi_{i}^{\prime}$. Then,

$$
\operatorname{Tr}\left[\Pi_{k}^{\prime} \cdots \Pi_{1}^{\prime} \rho \Pi_{1}^{\prime} \cdots \Pi_{k}^{\prime}\right] \geq \operatorname{Tr} \rho-4 \sum_{i=1}^{k} \operatorname{Tr}\left[\rho \Pi_{i}\right] .
$$

\section{Tilted span of subspaces}

Let $\mathcal{H}$ be a Hilbert space. Consider Hilbert spaces $\mathcal{H}_{j}, j \in$ $[l]$, each of dimension $\operatorname{dim} \mathcal{H}$. Define $\tilde{\mathcal{H}}:=\mathcal{H} \oplus \mathcal{H}_{1} \oplus \cdots \oplus \mathcal{H}_{l}$. Let $\mathcal{T}_{j}, j \in[l]$ be linear maps mapping $\mathcal{H}$ isometrically onto $\mathcal{H}_{j}$. For $j \in[l]$, let $0<\alpha_{j}<1$ and define linear maps $\mathcal{T}_{j, \alpha_{j}}: \mathcal{H} \rightarrow \tilde{\mathcal{H}}$ as

$$
\mathcal{T}_{j, \alpha_{j}}:=\sqrt{1-\alpha_{j}} \mathbb{1}_{\mathcal{H}}+\sqrt{\alpha_{j}} \mathcal{T}_{j},
$$

where $\mathbb{1}_{\mathcal{H}}$ is the identity embedding of $\mathcal{H}$ into $\tilde{\mathcal{H}}$. Observe that each $\mathcal{T}_{j, \alpha_{j}}$ is an isometric embedding of $\mathcal{H}$ into $\tilde{\mathcal{H}}$.

We now define the tilted span of a collection of subspaces, formalising the intuitive description in the introduction.

Definition 2 (Tilted span) Let $W_{1}, \ldots, W_{l}$ be subspaces of $\mathcal{H}$. Let $0<\alpha_{j}<1, j \in[l]$. The subspace $\mathcal{T}_{j, \alpha_{j}}\left(W_{j}\right) \leq \tilde{\mathcal{H}}$ will be called the $\alpha_{j}$-tilt of $W_{j}$ along the jth direction. The subspace $\mathbf{W}_{\left(\alpha_{1}, \ldots, \alpha_{l}\right)}$ of $\tilde{\mathcal{H}}$ defined by

$$
\mathbf{W}_{\left(\alpha_{1}, \ldots, \alpha_{l}\right)}:=\bigoplus_{j=1}^{l} \mathcal{T}_{j, \alpha_{j}}\left(W_{j}\right)
$$

is called the $\left(\alpha_{1}, \ldots, \alpha_{l}\right)$-tilted span of $W_{1}, \ldots, W_{l}$. If $\alpha_{j}=\alpha$ for all $j \in[l]$, we write $\mathbf{W}_{\alpha}$ and call it the $\alpha$-tilted span of $W_{1}, \ldots, W_{l}$.

The effect of tilting the subspaces $W_{1}, \ldots, W_{l}$ along $l$ orthogonal directions is to increase the separation between them, in a sense made precise by the following proposition.
Proposition 2 Let $0<\alpha \leq 1$. Let $|h\rangle \in \mathcal{H}$ be a unit vector. Let $W_{1}, \ldots, W_{l}$ be subspaces of $\mathcal{H}$. For $j \in[l]$, define $\epsilon_{j}:=$ $\| \Pi_{W_{j}}|h\rangle \|_{2}^{2}$ and let $0<\alpha_{j}<1$. Define $\alpha:=\min _{j: j \in[l]} \alpha_{j}$. Then,

$$
\max _{j: j \in[l]}\left(1-\alpha_{j}\right) \epsilon_{j} \leq \| \Pi_{\mathbf{W}_{\left(\alpha_{1}, \ldots, \alpha_{l}\right)}}|h\rangle \|_{2}^{2} \leq \frac{1-\alpha}{\alpha} \sum_{j=1}^{l} \epsilon_{j} .
$$

Proof: The lower bound follows trivially since the projection of $|h\rangle$ onto the $j$ th summand space in the definition of $\mathbf{W}_{\left(\alpha_{1}, \ldots, \alpha_{l}\right)}$ is of length $\sqrt{\epsilon_{j}\left(1-\alpha_{j}\right)}$.

We now prove the upper bound. Define $h^{\prime}:=\Pi_{\mathbf{W}_{\left(\alpha_{1}, \ldots, \alpha_{l}\right)}}|h\rangle$. Since $h^{\prime} \in \mathbf{W}_{\left(\alpha_{1}, \ldots, \alpha_{l}\right)}$, let $h^{\prime}=\sum_{j=1}^{l} \lambda_{j}\left|x_{j}\right\rangle$ where $\lambda_{j} \in \mathbb{C}$, and $\left|x_{j}\right\rangle$ is a unit vector in $\mathcal{T}_{j, \alpha_{j}}\left(W_{j}\right)$ for $j \in[l]$. Let $\left|x_{j}\right\rangle=$ $\mathcal{T}_{j, \alpha_{j}}\left(\left|y_{j}\right\rangle\right)$, where $\left|y_{j}\right\rangle$ is a unit vector in $W_{j}$ and is uniquely determined by $\left|x_{j}\right\rangle$. Then,

$$
\begin{aligned}
\left\|h^{\prime}\right\|_{2}^{2} & =\| \sum_{j=1}^{l} \sqrt{1-\alpha_{j}} \lambda_{j}\left|y_{j}\right\rangle+\sum_{j=1}^{l} \sqrt{\alpha_{j}} \lambda_{j} \mathcal{T}_{j}\left(\left|y_{j}\right\rangle\right) \|_{2}^{2} \\
& \geq \| \sum_{j=1}^{l} \sqrt{\alpha_{j}} \lambda_{j} \mathcal{T}_{j}\left(\left|y_{j}\right\rangle\right) \|_{2}^{2} \\
& \geq \alpha \sum_{j=1}^{l}\left|\lambda_{j}\right|^{2} .
\end{aligned}
$$

We also have

$\left\|h^{\prime}\right\|_{2}^{2}=\left|\left\langle h \mid h^{\prime}\right\rangle\right|=\left|\sum_{j=1}^{l} \lambda_{j}\left\langle h \mid x_{j}\right\rangle\right| \leq \sqrt{\sum_{j=1}^{l}\left|\lambda_{j}\right|^{2}} \cdot \sqrt{\sum_{j=1}^{l}\left|\left\langle h \mid x_{j}\right\rangle\right|^{2}}$.

This implies that

$$
\begin{aligned}
\alpha \sqrt{\sum_{j=1}^{l}\left|\lambda_{j}\right|^{2}} & \leq \sqrt{\sum_{j=1}^{l}\left|\left\langle h \mid x_{j}\right\rangle\right|^{2}}=\sqrt{\sum_{j=1}^{l}\left(1-\alpha_{j}\right)\left|\left\langle h \mid y_{j}\right\rangle\right|^{2}} \\
& =\sqrt{\sum_{j=1}^{l}\left(1-\alpha_{j}\right)\left|\left\langle h\left|\Pi_{W_{j}}\right| y_{j}\right\rangle\right|^{2}} \\
& \leq \sqrt{\sum_{j=1}^{l}\left(1-\alpha_{j}\right) \| \Pi_{W_{j}}|h\rangle \|_{2}^{2}} \\
& \leq \sqrt{1-\alpha} \sqrt{\sum_{j=1}^{l} \epsilon_{j}}
\end{aligned}
$$

Thus,

$$
\begin{aligned}
\left\|h^{\prime}\right\|_{2}^{2} & \leq \sqrt{\sum_{j=1}^{l}\left|\lambda_{j}\right|^{2}} \cdot \sqrt{\sum_{j=1}^{l}\left|\left\langle h \mid x_{j}\right\rangle\right|^{2}} \leq \frac{1}{\alpha} \sum_{j=1}^{l}\left|\left\langle h \mid x_{j}\right\rangle\right|^{2} \\
& \leq \frac{1-\alpha}{\alpha} \sum_{j=1}^{l} \epsilon_{j} .
\end{aligned}
$$

This proves the desired upper bound on $\left\|h^{\prime}\right\|_{2}^{2}$ and completes the proof of the proposition.

As a corollary, we now prove a small extension of Proposition 2 
Corollary 1 Let $|h\rangle \in \mathcal{H}$ be a unit vector. Let $W_{0}, W_{1}, \ldots, W_{l}$ be subspaces of $\mathcal{H}$. For $0 \leq j \leq l$, define $\epsilon_{j}:=\| \Pi_{W_{j}}|h\rangle \|_{2}^{2}$. Let $0<\alpha<1 / 3$. Define the subspace $\mathbf{W}$ of $\tilde{\mathcal{H}}$ as $\mathbf{W}:=\mathbf{W}_{\alpha}+W_{0}$. Define $\epsilon:=\frac{1-\alpha}{\alpha} \sum_{j=1}^{l} \epsilon_{j}$. Then,

$$
\max \left\{\epsilon_{0},(1-\alpha)\left(\max _{j: 1 \leq j \leq l} \epsilon_{j}\right)\right\} \leq \| \Pi_{\mathbf{W}}|h\rangle \|_{2}^{2} \leq \frac{3 l}{\alpha}\left(\epsilon_{0}+\epsilon\right) .
$$

Proof: As also remarked in the proof of Proposition 2, the lower bound is immediate. Hence, we concentrate on proving the upper bound.

It is easy to see that the largest inner product between a vector $|v\rangle \in \mathcal{H}$ and a vector $|w\rangle \in+_{j=1}^{l} \mathcal{T}_{j, \alpha}(\mathcal{H})$ is given by

$$
|w\rangle=\frac{1}{\sqrt{l^{2}(1-\alpha)+l \alpha}}\left(l \sqrt{1-\alpha}|v\rangle+\sqrt{\alpha} \sum_{j=1}^{l} \mathcal{T}_{j}(|v\rangle)\right),
$$

with inner product equal to

$$
\frac{\sqrt{l(1-\alpha)}}{\sqrt{l(1-\alpha)+\alpha}} \leq 1-\frac{\alpha}{3 l} \text {. }
$$

Define $h^{\prime}:=\Pi_{\mathbf{W}}|h\rangle$. Since $h^{\prime} \in \mathbf{W}$, let $h^{\prime}=h_{0}^{\prime}+h_{\alpha}^{\prime}$, where $h_{0}^{\prime} \in W_{0}$ and $h_{\alpha}^{\prime} \in \mathbf{W}_{\alpha}$. Then,

$$
\begin{aligned}
\left\|h^{\prime}\right\|_{2}^{2} & =\left\|h_{0}^{\prime}\right\|_{2}^{2}+\left\|h_{\alpha}^{\prime}\right\|_{2}^{2}+2 \mathfrak{R}\left(\left\langle h_{0}^{\prime} \mid h_{\alpha}^{\prime}\right\rangle\right) \\
& \geq\left\|h_{0}^{\prime}\right\|_{2}^{2}+\left\|h_{\alpha}^{\prime}\right\|_{2}^{2}-2\left\|h_{0}^{\prime}\right\|_{2}\left\|h_{\alpha}^{\prime}\right\|_{2}\left(1-\frac{\alpha}{3 l}\right) \\
& \geq\left(\left\|h_{0}^{\prime}\right\|_{2}^{2}+\left\|h_{\alpha}^{\prime}\right\|_{2}^{2}\right) \frac{\alpha}{3 l},
\end{aligned}
$$

where, in the last inequality, we used the fact that $2 x y \leq$ $x^{2}+y^{2}$ for real numbers $x, y$. On the other hand,

$$
\begin{aligned}
\left\|h^{\prime}\right\|_{2}^{2} & =\left|\left\langle h \mid h^{\prime}\right\rangle\right| \\
& \leq\left|\left\langle h \mid h_{0}^{\prime}\right\rangle\right|+\left|\left\langle h \mid h_{\alpha}^{\prime}\right\rangle\right| \\
& \leq\left\|h_{0}^{\prime}\right\|_{2} \sqrt{\epsilon_{0}}+\left\|h_{\alpha}^{\prime}\right\|_{2} \sqrt{\epsilon} \\
& \leq \sqrt{\left\|h_{0}^{\prime}\right\|_{2}^{2}+\left\|h_{\alpha}^{\prime}\right\|_{2}^{2}} \cdot \sqrt{\epsilon_{0}+\epsilon}
\end{aligned}
$$

In the second inequality above, we used the properties that $\| \Pi_{\mathbf{W}_{\alpha}}|h\rangle \|_{2}^{2} \leq \epsilon$ and $\| \Pi_{W_{0}}|h\rangle \|_{2}^{2}=\epsilon_{0}$. We thus get

$$
\sqrt{\left\|h_{0}^{\prime}\right\|_{2}^{2}+\left\|h_{\alpha}^{\prime}\right\|_{2}^{2}} \leq \frac{3 l}{\alpha} \sqrt{\epsilon_{0}+\epsilon}
$$

which means

$$
\left\|h^{\prime}\right\|_{2}^{2} \leq \frac{3 l}{\alpha}\left(\epsilon_{0}+\epsilon\right)
$$

This finishes the proof of the corollary.

\section{Remark:}

1. An easy corollary of Proposition 2 is a union bound for projectors that improves upon the union bound proved in Anshu, Jain and Warsi's paper [25, Lemma 3] on the entanglement assisted compound quantum channel.

2. Proposition 2 also gives an easier proof of the main result of Harrow, Lin and Montanaro's paper [26, Corollary 11] which they and earlier authors have referred to as a 'Quantum OR bound' for projectors. The 'OR projector' constructed by Proposition 2 improves the parameters of Harrow et al's construction and is more efficient to implement.
Corollary 2 Let $\epsilon, \alpha>0$. For $i \in[l]$, let $\rho_{i}$ be a quantum state and $\Pi_{i}$ be an orthogonal projector in $\mathcal{H}$ such that $\operatorname{Tr}\left[\Pi_{i} \rho_{i}\right] \geq 1-\epsilon$. Let $\tilde{\mathcal{H}}$ be defined as in Definition 2 Then there is a projector $\hat{\Pi} \in \tilde{\mathcal{H}}$ such that $\operatorname{Tr}\left[\hat{\Pi} \rho_{i}\right] \geq 1-\epsilon-\alpha$ for all $i \in[l]$, and, for all states $\sigma \in \mathcal{H}$,

$$
\operatorname{Tr}[\hat{\Pi} \sigma] \leq \frac{1-\alpha}{\alpha} \sum_{i \in[l]} \operatorname{Tr}\left[\Pi_{i} \sigma\right] .
$$

Suppose $\operatorname{Tr}\left[\Pi_{i} \sigma\right] \leq \theta$ for all $i \in[l]$. Then the upper bound promised by the above corollary is $\frac{l \theta}{\alpha}$ whereas the upper bound given by Lemma 3 of [25] is only $l \theta\left(\frac{2}{\alpha^{2}}\right)^{\log (2 l)}$. Thus, the above corollary leads to corresponding improvements in the achievable rates for the various settings considered in [25].

It is interesting to consider the analogoue of Proposition 2 in classical probability. One can think of the subspaces $W_{j}$, $j \in[l]$ as a set of $l$ events, the vector $|h\rangle$ as the classical state of a system, and $\epsilon_{j}$ to be the probablity of the $j$ th event occuring. Then the probability of at least one of the events occuring is lower bounded by $\max _{j: j \in[l]} \epsilon_{j}$ and upper bounded by $\sum_{j=1}^{l} \epsilon_{j}$. This is nothing but the fundamental union bound of classical probability. Thus, the above proposition almost recovers the classical performance of the union bound in the context of quantum probability.

However the above proposition still falls short of defining an intersection projector satisfying Properties 1, 2, 3, 4 for the cq-MAC. For $j \in[l]$, let $\Pi_{j}$ be an orthogonal projection. We would like to define a non-trivial intersection of the projectors $\Pi_{j}, j \in[l]$. Define a new projector $\hat{\Pi}$ as the projection onto the orthogonal complement of the tilted span $\mathbf{W}_{\alpha}$ of $W_{j}, j \in[l]$, where $W_{j}$ is taken to be the support of $\Pi_{j}$. Let $\rho$ be a quantum state and $\epsilon_{j}:=\operatorname{Tr}\left[\Pi_{j} \rho\right]$. Then it is easy to see that

$$
\operatorname{Tr}[\hat{\Pi} \rho] \geq 1-\frac{1-\alpha}{\alpha} \sum_{j=1}^{l} \epsilon_{j} .
$$

This is tolerable for most applications of the joint typicality lemma. In fact, it allows us to prove a good enough version of Property 1 for the cq-MAC taking $\rho=\rho^{X Y Z}, l=3, \Pi_{1}:=\Pi^{X}$, $\Pi_{2}:=\Pi^{Y}, \Pi_{3}:=\Pi^{X, Y}$.

Now consider states $\sigma_{i}, i \in[l]$. Let $2^{-k_{i}}:=\operatorname{Tr}\left[\Pi_{i} \sigma_{i}\right]$. For the cq-MAC, we have $l=3, \sigma_{1}:=\rho^{Y Z} \otimes \rho^{X}, \sigma_{2}:=\rho^{X Z} \otimes \rho^{Y}$, $\sigma_{3}:=\rho^{X} \otimes \rho^{Y} \otimes \rho^{Z}$. For the upper bound, the best that one can prove is

$$
\operatorname{Tr}\left[\hat{\Pi} \sigma_{i}\right] \leq \alpha\left(1-2^{-k_{i}}\right)+2^{-k_{i}}
$$

The additive term of nearly $\alpha$ makes the upper bound insufficient for applicatioins of the joint typicality lemma. In particular, it removes any hope of proving even approximate versions of Properties 2, 3, 4 for the cq-MAC.

However, for a $k$-partite state $\rho^{A_{1} \cdots A_{k}}$, and a collection of $k$-partite states $\sigma_{i}:=\rho^{A_{S_{i}}} \otimes \rho^{A_{S_{i}}},\{\} \neq S_{i} \subset[k], i \in[l]$, it turns out that we can do better and indeed come up with a notion of intersection projector that is strong enough to prove a quantum joint typicality lemma. For this, as discussed in the introduction, we have to do a smoothing of $\rho$ to $\rho^{\prime}$. The smoothing is achieved by a carefully chosen tilt of $\rho$. This 
makes $\sigma_{i}^{\prime}:=\left(\rho^{\prime}\right)^{A_{S_{i}}} \otimes\left(\rho^{\prime}\right)^{A_{S_{i}}}$ extremely close to a certain tilted version of $\sigma_{i}$. However, it turns out that the tilt of $\sigma_{i}$ is not only along the so-called $\left(S_{i}, \bar{S}_{i}\right)$ th direction but also along the directions corresponding to subpartitions refining $\left(S_{i}, \bar{S}_{i}\right)$. Thus, by taking a linear extension of the partial order of subpartitions of $[k]$ under refinement, we are led to consider a tilting scheme where $\sigma_{i}$ is tilted along the directions $1, \ldots, i$. This tilting scheme is described by an upper triangular tilting matrix $A$ whose $(i j)$ th entry $\alpha_{i j}$ denotes the tilt along the $i$ th direction for $\sigma_{j}$ when $i \leq j$. Hence, we have to formally define the $A$-tilted span of $W_{1}, \ldots, W_{l}$, and prove a union bound for it.

Let $0 \leq \alpha_{i j} \leq 1$, where $i, j \in[l]$. Define the $l \times l$ matrix $A:=\left(\alpha_{i j}\right)$. Suppose $A$ is upper triangular and diagonal dominated, that is, $\alpha_{i j}=0$ for $i>j$ and $\alpha_{i i} \geq \alpha_{i j}$ for all $i \leq j$. Furthermore, suppose $A$ is substochastic, that is, for all $j, \sum_{i=1}^{j} \alpha_{i j} \leq 1$. If the last inequality holds with equality, we say that $A$ is stochastic. For $j \in[l]$, define linear maps $\mathcal{T}_{j, A}: \mathcal{H} \rightarrow \tilde{\mathcal{H}}$ as

$$
\mathcal{T}_{j, A}:=\sqrt{1-\sum_{i=1}^{j} \alpha_{i j}} \mathbf{1}_{\mathcal{H}}+\sum_{i=1}^{j} \sqrt{\alpha_{i j}} \mathcal{T}_{i} .
$$

Observe that each $\mathcal{T}_{j, A}$ is an isometric embedding of $\mathcal{H}$ into $\tilde{\mathcal{H}}$, which we shall refer to as the $A$-tilt along the $j$ th direction. We shall call $A$ as the tilting matrix. A tilting matrix is always assumed to be upper triangular, diagonal dominated, and substochastic.

Definition 3 ( $A$-tilted span) Let $W_{1}, \ldots, W_{l}$ be subspaces of $\mathcal{H}$. The subspace $\mathcal{T}_{j, A}\left(W_{j}\right) \leq \tilde{\mathcal{H}}$ will be called the $A$-tilt of $W_{j}$ along the directions $1, \ldots, j$. The subspace $\mathbf{W}_{A}$ of $\tilde{\mathcal{H}}$ defined by

$$
\mathbf{W}_{A}:=\prod_{j=1}^{l} \mathcal{T}_{j, A}\left(W_{j}\right) .
$$

is called the A-tilted span of $W_{1}, \ldots, W_{l}$.

The effect of doing $A$-tilting of the subspaces $W_{1}, \ldots, W_{l}$ along $l$ orthogonal directions is to increase the separation between them, in a sense made precise by the following proposition.

Proposition 3 Let $A$ be an upper triangular diagonal domniated substochastic matrix. Let $|h\rangle \in \mathcal{H}$ be a unit vector. For $j \in[l]$, define $\epsilon_{j}:=\| \Pi_{W_{j}}|h\rangle \|_{2}^{2}$. Then,

$$
\begin{aligned}
\max _{j: j \in[l]} \epsilon_{j}\left(1-\sum_{i=1}^{j} \alpha_{i j}\right) & \leq \| \Pi_{\mathbf{W}_{A}}|h\rangle \|_{2}^{2} \\
& \leq\left(\sum_{j=1}^{l} \sqrt{\epsilon_{j}}\left(\sum_{k=j}^{l} 2^{k-j} \alpha_{k k}^{-1 / 2}\right)\right)^{2} .
\end{aligned}
$$

In particular, if $\alpha_{j j} \geq \alpha$ for all $j \in[l]$, we can obtain an upper bound of

$$
\| \Pi_{\mathbf{W}_{A}}|h\rangle \|_{2}^{2} \leq \frac{2^{2 l+1}}{\alpha} \sum_{j=1}^{l} \epsilon_{j} .
$$

Proof: The lower bound follows trivially since the projection of $|h\rangle$ onto the $j$ th summand space in the definition of $\mathbf{W}_{A}$ is of length $\sqrt{\epsilon_{j}\left(1-\sum_{i=1}^{j} \alpha_{i j}\right)}$.

We now prove the upper bound. Define $h^{\prime}:=\Pi_{\mathbf{W}_{A}}|h\rangle$. Since $h^{\prime} \in \mathbf{W}_{A}$, let $h^{\prime}=\sum_{j=1}^{l} \lambda_{j}\left|x_{j}\right\rangle$ where $\lambda_{j} \in \mathbb{C}$, and $\left|x_{j}\right\rangle$ is a unit vector in $\mathcal{T}_{j, A}\left(W_{j}\right)$ for $j \in[l]$. Let $\left|x_{j}\right\rangle=\mathcal{T}_{j, A}\left(\left|y_{j}\right\rangle\right)$, where $\left|y_{j}\right\rangle$ is a unit vector in $W_{j}$ and is uniquely determined by $\left|x_{j}\right\rangle$. Since the spaces $\mathcal{H}_{j}, j \in[l]$ are orthogonal to $\mathcal{H}$, we have

$$
\begin{aligned}
\left\|h^{\prime}\right\|_{2} & =\| \sum_{j=1}^{l} \sqrt{1-\sum_{i=1}^{j} \alpha_{i j}} \lambda_{j}\left|y_{j}\right\rangle+\sum_{j=1}^{l} \sum_{i=1}^{j} \sqrt{\alpha_{i j}} \lambda_{j} \mathcal{T}_{i}\left(\left|y_{j}\right\rangle\right) \|_{2} \\
& \geq \| \sum_{j=1}^{l} \sum_{i=1}^{j} \sqrt{\alpha_{i j}} \lambda_{j} \mathcal{T}_{i}\left(\left|y_{j}\right\rangle\right) \|_{2} .
\end{aligned}
$$

We now prove by backward induction on $i$ that

$$
\left|\lambda_{i}\right| \leq\left\|h^{\prime}\right\|_{2}\left(\sum_{j=i}^{l} 2^{j-i} \alpha_{j j}^{-1 / 2}\right) .
$$

The base case of $i=l+1$ is vacuously true taking $\lambda_{l+1}=0$. Suppose the claim is true for $i+1$. We now prove it for $i$. Observe that

$$
\begin{aligned}
\sqrt{\alpha_{i i}}\left|\lambda_{i}\right|-\sum_{j=i+1}^{l} \sqrt{\alpha_{i j}}\left|\lambda_{j}\right| & \leq \| \sum_{j=i}^{l} \sqrt{\alpha_{i j}} \lambda_{j} \mathcal{T}_{i}\left(\left|y_{j}\right\rangle\right) \|_{2} \\
& \leq \| \sum_{i=1}^{l} \sum_{j=i}^{l} \sqrt{\alpha_{i j}} \lambda_{j} \mathcal{T}_{i}\left(\left|y_{j}\right\rangle\right) \|_{2} \\
& \leq\left\|h^{\prime}\right\|_{2},
\end{aligned}
$$

where we used the fact that $\mathcal{H}_{i}$ is orthogonal to $\mathcal{H}_{i^{\prime}}$ for all $i^{\prime} \neq$ $i$ in the second inequality. Using the induction hypothesis and the diagonal dominated property, we get

$$
\begin{aligned}
\left|\lambda_{i}\right| & \leq \frac{\left\|h^{\prime}\right\|_{2}}{\sqrt{\alpha_{i i}}}+\sum_{j=i+1}^{l} \frac{\sqrt{\alpha_{i j}}}{\sqrt{\alpha_{i i}}}\left|\lambda_{j}\right| \\
& \leq \frac{\left\|h^{\prime}\right\|_{2}}{\sqrt{\alpha_{i i}}}+\sum_{j=i+1}^{l}\left|\lambda_{j}\right| \\
& \leq\left\|h^{\prime}\right\|_{2}\left(\alpha_{i i}^{-1 / 2}+\sum_{j=i+1}^{l} \sum_{k=j}^{l} 2^{k-j} \alpha_{k k}^{-1 / 2}\right) .
\end{aligned}
$$

Rearranging, we get

$$
\begin{aligned}
\left|\lambda_{i}\right| & \leq\left\|h^{\prime}\right\|_{2}\left(\alpha_{i i}^{-1 / 2}+\sum_{k=i+1}^{l} \sum_{j=i+1}^{k} 2^{k-j} \alpha_{k k}^{-1 / 2}\right) \\
& \leq\left\|h^{\prime}\right\|_{2}\left(\alpha_{i i}^{-1 / 2}+\sum_{k=i+1}^{l} 2^{k-i} \alpha_{k k}^{-1 / 2}\right),
\end{aligned}
$$

which completes the proof of the inequality in (4) by induction. 
We also have

$$
\begin{aligned}
& \left\|h^{\prime}\right\|_{2}^{2}=\left|\left\langle h \mid h^{\prime}\right\rangle\right|=\left|\sum_{j=1}^{l} \lambda_{j}\left\langle h \mid x_{j}\right\rangle\right| \\
& \leq \sum_{j=1}^{l}\left|\lambda_{j}\right|\left|\left\langle h \mid x_{j}\right\rangle\right|=\sum_{j=1}^{l}\left|\lambda_{j}\right| \sqrt{1-\sum_{i=1}^{j} \alpha_{i j}}\left|\left\langle h \mid y_{j}\right\rangle\right| \\
& \leq \sum_{j=1}^{l}\left|\lambda_{j}\right|\left|\left\langle h\left|\Pi_{W_{j}}\right| y_{j}\right\rangle\right| \leq \sum_{j=1}^{l}\left|\lambda_{j}\right| \| \Pi_{W_{j}}\left|y_{j}\right\rangle \|_{2} \\
& =\sum_{j=1}^{l}\left|\lambda_{j}\right| \sqrt{\epsilon_{j}} \text {. }
\end{aligned}
$$

Combining the above inequality with the inequality in (Equation 4, we get

$$
\left\|h^{\prime}\right\|_{2} \leq \sum_{j=1}^{l}\left(\sum_{k=j}^{l} 2^{k-j} \alpha_{k k}^{-1 / 2}\right) \sqrt{\epsilon_{j}},
$$

which completes the proof of the proposition. The special case where $\alpha_{j j} \geq \alpha$ for all $j \in[l]$ follows via CauchySchwarz inequality.

\section{Remark:}

It is interesting to contrast the claims of Propositions 2 and 3. In the case where $\alpha_{j j}=\alpha$ for all $j \in[l]$ and $\alpha_{j j^{\prime}}=0$ for $j \neq j^{\prime}$, Proposition 2 gives an upper bound of $\frac{1-\alpha}{\alpha} \sum_{j=1}^{l} \epsilon_{j}$. On the other hand, Proposition 3 gives a worse upper bound of $\frac{2^{2 l+1}}{\alpha} \sum_{j=1}^{l} \epsilon_{j}$. The power of Proposition 3 lies in its generality. As discussed earlier, it turns out that we need the general setting of Proposition 3 in order to construct an intersection projector strong enough to prove the one-shot quantum joint typicality lemma. In other words, Proposition 3 can be thought of as a way to do intersection of hypothesis tests in the quantum setting. Proposition 2 will be used in situations where one needs to consider union of quantum hypothesis tests in the quantum setting. Such situations arise in several network information theoretic tasks on top of joint typicality requirements. Some concrete applications where both intersection and union of hypothesis tests are required can be found in the companion paper [11].

\section{Simplified one-shot inner bound for the classical quan- tum MAC}

As a warmup, we show how tilting, and smoothing and augmentation can be used to prove a simplified version of our one-shot inner bound for the multiple access channel with classical inputs and quantum output, called cq-MAC henceforth (see Fig. 3). In the asymptotic iid setting, Winter [9] used a standard successive cancellation argument to reduce the problem of finding inner bounds for the cq-MAC to the problem of finding inner bounds for the classical-quantum point-to-point channel. This allowed him to prove an optimal inner bound in the asymptotic iid setting. However in the one-shot setting, successive cancellation arguments give only a finite set of achievable rate tuples. In order to get a continuous rate region, we need to look for a simultaneous decoder for the cq-MAC. Fawzi et al [17] and Sen [10] did construct a simultaneous decoder for the two sender cq-MAC but their constructions, which were given in the asymptotic iid setting, are not known to work in the one-shot setting. Qi, Wang and Wilde [19] constructed a one-shot simultaneous decoder for the cq-MAC with an arbitrary number of senders, but their achievable rates restricted to the asymptotic iid setting are inferior to the optimal rates obtained by Winter. Thus, for more than two senders a simultaneous decoder for the cqMAC achieving optimal rates was hitherto unknown even in the asymptotic iid setting.

In this section, we construct a simultaneous decoder for the cq-MAC for two senders. The simplified one shot inner bound given in this section does not use a so-called 'time sharing random variable'. The full version with the time sharing random variable can be found in Section 6 We obtain an inner bound which turns out to be the natural oneshot analogue of Winter's inner bound, as well as the natural quantum analogue of the one-shot classical inner bound described in Section 1.2. In other words, our one-shot inner bound reduces to Winter's optimal inner bound in the asymptotic iid setting. A one-shot outer bound nearly matching our inner bound was shown recently by Anshu, Jain and Warsi [27]. That paper also gives another inner bound for the cq-MAC which, though nearly optimal in the one-shot setting, is not known to reduce to the standard asymptotic iid inner bound of Winter unlike our one-shot inner bound.

There are two senders Alice and Bob who would like to send classical messages $m_{1} \in\left[2^{R_{1}}\right], m_{2} \in 2^{R_{2}}$ to a receiver Charlie. There is a communication channel $\mathfrak{C}$ with two classical inputs and one quantum output called a cq-MAC connecting Alice and Bob to Charlie. The two input alphabets of $\mathfrak{C}$ will be denoted by $\mathcal{X}, \mathcal{Y}$ and the output Hilbert space by $\mathcal{Z}$. Let $0 \leq \epsilon \leq 1$. On getting message $m_{1}$, Alice encodes it as a letter $x\left(m_{1}\right) \in \mathcal{X}$ and feeds it to her channel input. Similarly on getting message $m_{2}$, Bob encodes it as a letter $y\left(m_{2}\right) \in \mathcal{Y}$ and feeds it to his channel input. The channel $\mathbb{C}$ outputs a quantum state $\rho_{x\left(m_{1}\right), y\left(m_{2}\right)}^{Z}$ in $\mathcal{Z}$. Charlie now has to try and guess the message pair $\left(m_{1}, m_{2}\right)$ from the channel output. We require that the probability of Charlie's decoding error averaged over the uniform distribution on the set of message pairs $\left(m_{1}, m_{2}\right) \in\left[2^{R_{1}}\right] \times\left[2^{R_{2}}\right]$ is at most $\epsilon$.

Fix independent probability distributions $p(x), p(y)$ on sets $\mathcal{X}, \mathcal{Y}$. Consider the classical-quantum state

$$
\rho^{X Y Z}:=\sum_{x, y} p(x) p(y)|x, y\rangle\left\langle x,\left.y\right|^{X Y} \otimes \rho_{x, y}^{Z} .\right.
$$

This state 'controls' the encoding and decoding performance for the channel c. Define

$$
\begin{aligned}
\rho_{x}^{Z} & :=\sum_{y} p(y) \rho_{x, y}^{Z}, \\
\rho_{y}^{Z} & :=\sum_{x} p(x) \rho_{x, y}^{Z},
\end{aligned}
$$




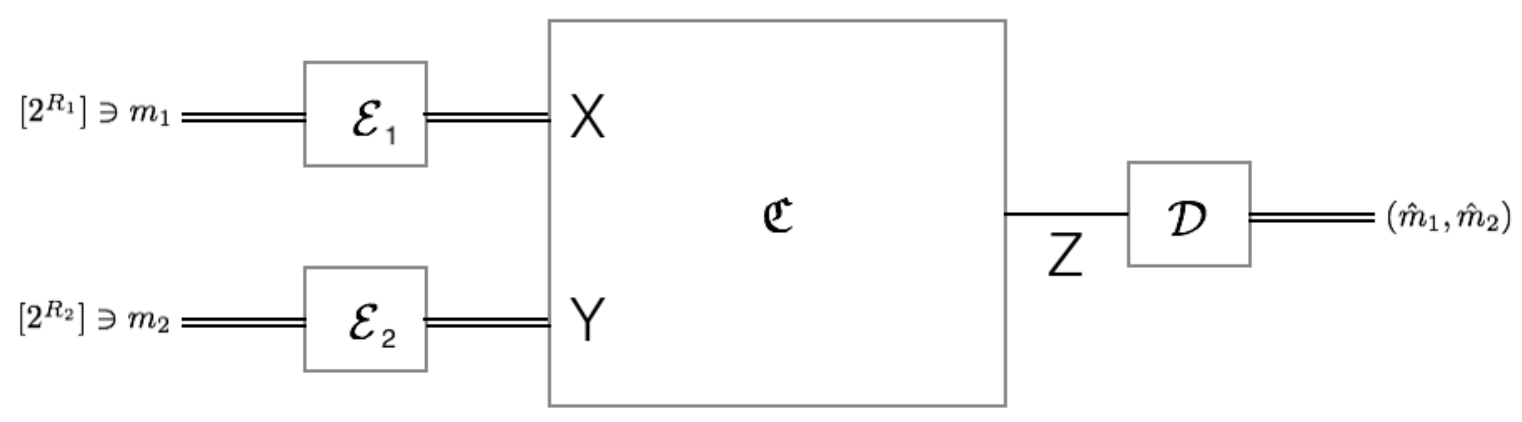

Figure 3. A classical quantum multiple access channel. Alice, Bob encode their respective messages $m_{1}, m_{2}$ into classical codewords and then feed them to the channel. Charlie applies a decoding superoperator to the quantum output of the channel to get his guess $\left(\hat{m}_{1}, \hat{m}_{2}\right)$ for the transmitted messages. $X, Y$ are the input alphabets and $Z$ is the output Hilbert space of the channel.

$$
\rho^{Z}:=\sum_{x, y} p(x) p(y) \rho_{x, y}^{Z} .
$$

Then,

$$
\begin{aligned}
\rho^{X Z} & =\sum_{x} p(x)|x\rangle\langle x| \otimes \rho_{x}^{Z}, \\
\rho^{Y Z} & :=\sum_{y} p(y)|y\rangle\langle y| \otimes \rho_{y}^{Z} .
\end{aligned}
$$

Consider a new alphabet, as well as Hilbert space, $\mathcal{L}$ and define the augmented systems $X^{\prime}:=\mathcal{X} \otimes \mathcal{L}, \mathcal{Y}^{\prime}:=\mathcal{Y} \otimes \mathcal{L}$, and the extended system $\mathcal{Z}^{\prime}$ as follows:

$$
\mathcal{Z}^{\prime}:=\left(\mathcal{Z} \otimes \mathbb{C}^{2}\right) \oplus\left(\mathcal{Z} \otimes \mathbb{C}^{2} \otimes \mathcal{L}^{X}\right) \oplus\left(\mathcal{Z} \otimes \mathbb{C}^{2} \otimes \mathcal{L}^{Y}\right),
$$

where the symbol $\oplus$ denotes orthogonal direct sum of spaces, $\mathcal{L}^{X}, \mathcal{L}^{Y}$ are distinct orthogonal spaces isomorphic to $\mathcal{L}$ labelled with mnemonic superscripts $X, Y$. The role of the mnemonics will become clear shortly when we described the tilted state. The aim of augmentation is to ensure a so-called smoothness property of states obtained by averaging over $\mathcal{X}^{\prime}$ or $\mathcal{Y}^{\prime}$ or both.

Consider a 'variant' cq-MAC $\mathbb{C}^{\prime}$ with input alphabets $\mathcal{X}^{\prime}$, $\boldsymbol{Y}^{\prime}$ and output Hilbert space $\mathcal{Z}^{\prime}$. On input $\left(x, l_{x}\right),\left(y, l_{y}\right)$ to $\mathfrak{C}^{\prime}$, the output of $\mathbb{C}^{\prime}$ is the state $\rho_{x, y}^{Z} \otimes|0\rangle\left\langle\left. 0\right|^{\mathbb{C}^{2}}\right.$. The output of $\mathbb{C}^{\prime}$ is taken to embed into the first summand in the definition of $\mathcal{Z}^{\prime}$ above. The classical quantum state 'controlling' the encoding and decoding for $\mathbb{C}^{\prime}$ is nothing but $\rho^{X Y Z} \otimes|0\rangle\left\langle\left. 0\right|^{\mathbb{C}^{2}} \otimes\right.$ $\frac{\mathbb{1}^{\mathcal{L}^{\otimes 2}}}{|\mathcal{L}|^{2}}$. The channel $\mathfrak{C}^{\prime}$ can be trivally obtained from channel $\mathfrak{C}$. The expected average decoding error for $\mathfrak{C}^{\prime}$ is the same as the expected average decoding error for $\mathbb{C}$ for the same rate pair $\left(R_{1}, R_{2}\right)$. In fact, an encoding-decoding scheme for $\mathbb{C}^{\prime}$ immediately gives an encoding-decoding scheme for $\mathfrak{C}$ with the same rate pair $\left(R_{1}, R_{2}\right)$ and the same decoding error.

Let $0 \leq \delta \leq 1 / 10$. Define $\hat{\mathcal{Z}}:=\mathcal{Z} \otimes \mathbb{C}^{2}$. Let $l_{x} \in \mathcal{L}^{X}$, $l_{y} \in \mathcal{L}^{Y}$. Consider the tilting map $\mathcal{T}_{X ; l_{x}, \delta}: \hat{\mathcal{Z}} \rightarrow \hat{\mathcal{Z}} \oplus\left(\hat{\mathcal{Z}} \otimes \mathcal{L}^{X}\right)$ defined as

$$
\mathcal{T}_{X ; l_{x}, \delta}:|h\rangle \mapsto \frac{1}{\sqrt{1+\delta^{2}}}\left(|h\rangle+\delta|h\rangle\left|l_{x}\right\rangle\right)
$$

Define the tilting map $\mathcal{T}_{Y: l_{y}, \delta}$ analogously. Define the tilting $\operatorname{map} \mathcal{T}_{X Y ; l_{x}, l_{y}, \delta}: \hat{\mathcal{Z}} \rightarrow \mathcal{Z}^{\prime}$ as

$$
\mathcal{T}_{X Y ; l_{x}, l_{y}, \delta}:|h\rangle \mapsto \frac{1}{\sqrt{1+2 \delta^{2}}}\left(|h\rangle+\delta|h\rangle\left|l_{x}\right\rangle+\delta|h\rangle\left|l_{y}\right\rangle\right) .
$$

Next, consider a 'perturbed' cq-MAC $\mathfrak{C}^{\prime \prime}$ with the same input alphabets and output Hilbert space as in $\mathbb{C}^{\prime}$ (see Fig. 4). However, on input $\left(x, l_{x}\right),\left(y, l_{y}\right)$ the output is the state

$$
\left(\rho^{\prime}\right)_{\left(x, l_{x}\right),\left(y, l_{y}\right), \delta}^{Z^{\prime}}:=\mathcal{T}_{X Y ; l_{x}, l_{y}, \delta}\left(\rho_{x, y}^{Z} \otimes|0\rangle\left\langle\left. 0\right|^{\mathbb{C}^{2}}\right),\right.
$$

where the map $\mathcal{T}_{X Y ; l_{x}, l_{y}, \delta}$ acts on a mixed state by acting on each pure state in the mixture individually. Consider the classical quantum state

$$
\begin{gathered}
\left(\rho^{\prime}\right)^{X^{\prime} Y^{\prime} Z^{\prime}} \\
:=|\mathcal{L}|^{-2} \sum_{x, y, l_{x}, l_{y}} p(x) p(y)\left|x, l_{x}\right\rangle\left\langle x,\left.l_{x}\right|^{X^{\prime}} \otimes \mid y, l_{y}\right\rangle\left\langle y,\left.l_{y}\right|^{Y^{\prime}}\right. \\
\otimes\left(\rho^{\prime}\right)_{\left(x, l_{x}\right),\left(y, l_{y}\right), \delta}^{Z^{\prime}} .
\end{gathered}
$$

This state 'controls' the encoding and decoding performance for the channel $\mathbb{C}^{\prime \prime}$.

It is now easy to see that

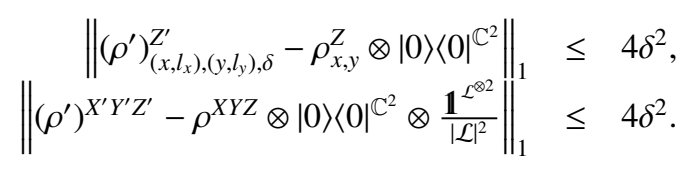

Thus, the expected average decoding error for $\mathbb{C}^{\prime \prime}$ is at most the expected average decoding error for $\mathfrak{C}^{\prime}$, which is also the same as the expected average decoding error for $\mathfrak{C}$, plus $2 \delta^{2}$, for the same rate pair $\left(R_{1}, R_{2}\right)$, and the same decoding strategy.

Consider the following randomised construction of a codebook $C$ for Alice and Bob for communication over the channel $\mathfrak{C}^{\prime \prime}$. Fix probability distributions $p(x), p(y)$ on sets $\mathcal{X}, \mathcal{Y}$. For all $m_{1} \in\left[2^{R_{1}}\right]$, choose $\left(x, l_{x}\right)\left(m_{1}\right) \in \mathcal{X} \times \mathcal{L}$ independently according to the product of the distribution $p(x)$ on $\mathcal{X}$ and 


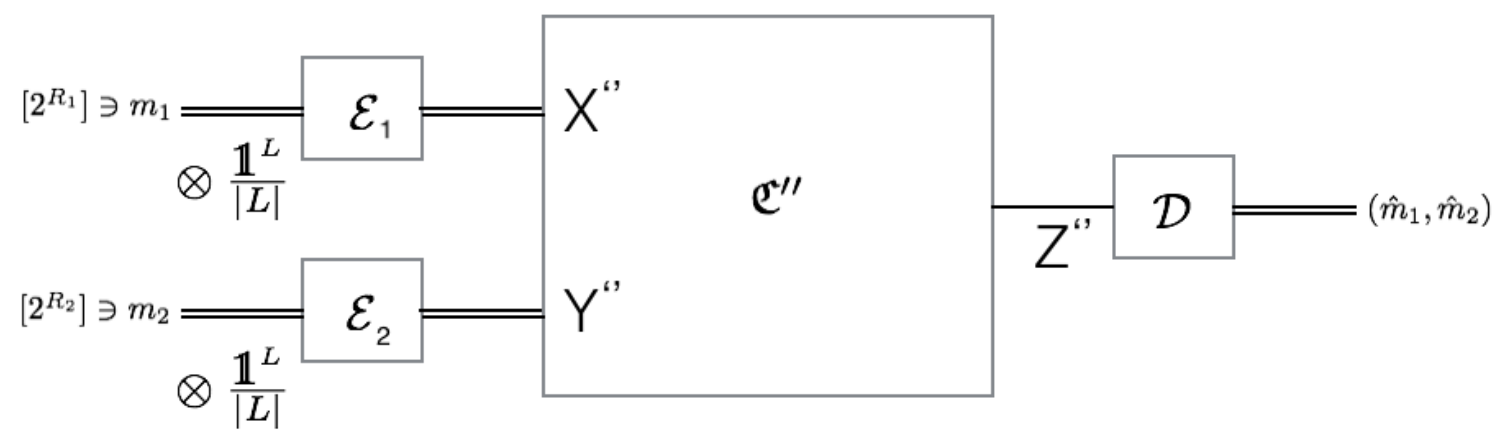

Figure 4. The perturbation of the classical quantum multiple access channel $\mathfrak{C}$ of Fig. 3 Alice, Bob encode their respective messages $m_{1}, m_{2}$ into classical codewords, tensor the codewords with completely mixed states over $L$, and then feed them to the perturbed channel $\mathfrak{C}^{\prime \prime}$. Charlie applies a decoding superoperator to the quantum output of the channel to get his guess $\left(\hat{m}_{1}, \hat{m}_{2}\right)$ for the transmitted messages. $X^{\prime \prime}, Y^{\prime \prime}$ are the input alphabets and $Z^{\prime \prime}$ is the output Hilbert space of the channel. A rate pair that is achievable for the perturbed channel $\mathfrak{C}^{\prime \prime}$ is also achievable for the original channel $\mathfrak{C}$ with at most a slight increase in error probability.

the uniform distribution on $\mathcal{L}$. Similarly for all $m_{2} \in\left[2^{R_{2}}\right]$, choose $\left(y, l_{y}\right)\left(m_{2}\right) \in \mathcal{Y} \times \mathcal{L}$ independently according to the product of the distribution $p(y)$ on $\mathcal{Y}$ and the uniform distribution on $\mathcal{L}$.

Let $\epsilon>0$. Consider the optimising POVM element $\left(\Pi^{\prime \prime}\right)_{X}^{X Y Z}$ in the Hilbert space $\mathcal{X} \otimes \mathcal{Y} \otimes \mathcal{Z}$ arising in the definition of $I_{H}^{\epsilon}(Y: X Z)$. Without loss of generality $\Pi_{X}^{X Y Z}$ is of the form

$$
\left(\Pi^{\prime \prime}\right)_{X}^{X Y Z}=\sum_{x, y}|x\rangle\left\langle\left. x\right|^{X} \otimes \mid y\right\rangle\left\langle\left. y\right|^{Y} \otimes\left(\Pi^{\prime \prime}\right)_{X ; x, y}^{Z} .\right.
$$

Similarly we can define, for each $(x, y) \in \mathcal{X} \times \mathcal{Y}$, POVM elements $\left(\Pi^{\prime \prime}\right)_{Y ; x, y}^{Z},\left(\Pi^{\prime \prime}\right)_{x, y}^{Z}$ arising in the definitions of $I_{H}^{\epsilon}(X$ : $Y Z), I_{H}^{\epsilon}(X Y: Z)$. Then

$$
\begin{aligned}
\operatorname{Tr}\left[\left(\Pi^{\prime \prime}\right)_{X}^{X Y Z} \rho^{X Y Z}\right] & \geq 1-\epsilon, \\
\operatorname{Tr}\left[\left(\Pi^{\prime \prime}\right)_{X}^{X Y Z}\left(\rho^{Y} \otimes \rho^{X Z}\right)\right] & \leq 2^{-I_{H}^{\epsilon}(X: Y Z)}, \\
\operatorname{Tr}\left[\left(\Pi^{\prime \prime}\right)_{Y}^{X Y} \rho^{X Y Z}\right] & \geq 1-\epsilon, \\
\operatorname{Tr}\left[\left(\Pi^{\prime \prime}\right)_{Y}^{X Y Z}\left(\rho^{X} \otimes \rho^{Y Z}\right)\right] & \leq 2^{-I_{H}^{\epsilon}(Y: X Z)}, \\
\operatorname{Tr}\left[\left(\Pi^{\prime \prime}\right)^{X Y Z} \rho^{X Y Z}\right] & \geq 1-\epsilon, \\
\operatorname{Tr}\left[\left(\Pi^{\prime \prime}\right)^{X Y Z}\left(\rho^{X Y} \otimes \rho^{Z}\right)\right] & \leq 2^{-I_{H}^{\epsilon}(X Y: Z)} .
\end{aligned}
$$

By Fact 2 there are orthogonal projections $\Pi_{X ; x, y}^{\hat{Z}}, \Pi_{Y ; x, y}^{\hat{Z}}$, $\Pi_{x, y}^{\hat{Z}}$ in $\mathcal{Z}^{\prime}$ that give the same measurement probability on states $\sigma^{Z} \otimes|0\rangle\left\langle\left. 0\right|^{\mathbb{C}^{2}}\right.$ that POVM elements $\left(\Pi^{\prime \prime}\right)_{X ; x, y}^{Z},\left(\Pi^{\prime \prime}\right)_{Y ; x, y}^{Z}$, $\left(\Pi^{\prime \prime}\right)_{x, y}^{Z}$ give on states $\sigma^{Z}$. Let $W_{X ; x, y}$ denote the orthogonal complement of the support of $\Pi_{X ; x, y}^{\hat{Z}}$ in $\hat{\mathcal{Z}}$. Define $W_{Y ; x, y}, W_{x, y}$ analogously. Define the tilted spaces

$W_{X ;\left(x, l_{x}\right),\left(y, l_{y}\right), \delta}^{\prime}:=\mathcal{T}_{X ; l_{x}, \delta}\left(W_{X ; x, y}\right), W_{Y ;\left(x, l_{x}\right),\left(y, l_{y}\right), \delta}^{\prime}:=\mathcal{T}_{Y ; l_{y}, \delta}\left(W_{Y ; x, y}\right)$, residing in the Hilbert space $\mathcal{Z}^{\prime}$. Define the subspace

$$
W_{\left(x, l_{x}\right),\left(y, l_{y}\right), \delta}^{\prime}:=W_{X ;\left(x, l_{x}\right),\left(y, l_{y}\right), \delta}^{\prime}+W_{Y ;\left(x, l_{x}\right),\left(y, l_{y}\right), \delta}^{\prime}+W_{x, y},
$$

and $\left(\Pi^{\prime}\right)_{W_{\left(x, l_{x}\right),\left(y, l_{y}\right), \delta}^{Z^{\prime}}}^{\prime}$ to be the orthogonal projection in $\mathcal{Z}^{\prime}$ onto $W_{\left(x, l_{x}\right),\left(y, l_{y}\right), \delta}^{\prime}$. Let $\Pi_{\hat{\mathcal{Z}}}^{Z^{\prime}}$ be the orthogonal projection in $\mathcal{Z}^{\prime}$ onto
$\hat{\mathcal{Z}}$. Then define the POVM element $\left(\Pi^{\prime}\right)_{\left(x, l_{x}\right),\left(y, l_{y}\right), \delta}^{Z^{\prime}}$ in $\mathcal{Z}^{\prime}$ to be $\left(\Pi^{\prime}\right)_{\left(x, l_{x}\right),\left(y, l_{y}\right), \delta}^{Z^{\prime}}:=\left(\mathbb{1}^{Z^{\prime}}-\left(\Pi^{\prime}\right)_{W_{\left(x, l_{x}\right),\left(y, l_{y}\right), \delta}^{Z^{\prime}}}^{Z^{\prime}} \Pi_{\hat{Z}^{\prime}}^{Z^{\prime}}\left(\mathbb{1}^{Z^{\prime}}-\left(\Pi^{\prime}\right)_{\left.W_{\left(x, l_{x}\right),\left(y, l_{y}\right), \delta}^{Z^{\prime}}\right)}^{Z^{\prime}}\right)\right.$.

Define the POVM element

$$
\begin{aligned}
& \left(\Pi^{\prime}\right)^{X^{\prime} Y^{\prime} Z^{\prime}} \\
& :=\sum_{x, y, l_{x}, l_{y}}\left|x, l_{x}\right\rangle\left\langle x,\left.l_{x}\right|^{X^{\prime}} \otimes \mid y, l_{y}\right\rangle\left\langle y,\left.l_{y}\right|^{Y^{\prime}} \otimes\left(\Pi^{\prime}\right)_{\left(x, l_{x}\right),\left(y, l_{y}\right), \delta}^{Z^{\prime}} .\right.
\end{aligned}
$$

By Equations 5, 6, Fact 3 and Corollary 1, we get

$$
\begin{gathered}
\operatorname{Tr}\left[\left(\mathbb{1}^{X^{\prime} Y^{\prime} Z^{\prime}}-\left(\Pi^{\prime}\right)^{X^{\prime} Y^{\prime} Z^{\prime}}\right)\left(\rho^{\prime}\right)^{X^{\prime} Y^{\prime} Z^{\prime}}\right] \\
\left.=\operatorname{Tr}\left(\rho^{\prime}\right)^{X^{\prime} Y^{\prime} Z^{\prime}}-\operatorname{Tr}\left[\left(\Pi^{\prime}\right)^{X^{\prime} Y^{\prime} Z^{\prime}}\right)\left(\rho^{\prime}\right)^{X^{\prime} Y^{\prime} Z^{\prime}}\right] \\
=|\mathcal{L}|^{-2} \sum_{x, y, l_{x}, l_{y}} p(x) p(y)\left(\operatorname{Tr}\left(\rho^{\prime}\right)_{\left(x, l_{x}\right),\left(y, l_{y}\right), \delta}^{Z^{\prime}}\right. \\
\left.\left.-\operatorname{Tr}\left[\left(\Pi^{\prime}\right)^{Z^{\prime}}\right)_{\left(x, l_{x}\right),\left(y, l_{y}\right), \delta}\left(\rho^{\prime}\right)_{\left(x, l_{x}\right),\left(y, l_{y}\right), \delta}^{Z^{\prime}}\right]\right) \\
=|\mathcal{L}|^{-2} \sum_{x, y, l_{x}, l_{y}} p(x) p(y)\left(\operatorname{Tr}\left(\rho^{\prime}\right)_{\left(x, l_{x}\right),\left(y, l_{y}\right), \delta}^{Z^{\prime}}\right. \\
-\operatorname{Tr}\left[\Pi_{\hat{\mathcal{Z}}}^{Z^{\prime}}\left(\mathbb{1}^{Z^{\prime}}-\left(\Pi^{\prime}\right)_{\left.W_{\left(x, l_{x}\right),\left(y, l_{y}\right), \delta}^{Z^{\prime}}\right)}\right)\right. \\
\left(\rho^{\prime}\right)_{\left(x, l_{x}\right),\left(y, l_{y}\right), \delta}^{Z^{\prime}} \\
\left(\mathbb{1}^{Z^{\prime}}-\left(\Pi^{\prime}\right)_{\left.\left.\left.W_{\left(x, l_{x}\right),\left(y, l_{y}\right), \delta}^{Z^{\prime}}\right) \Pi_{\hat{Z}^{\prime}}^{Z^{\prime}}\right]\right)}\right.
\end{gathered}
$$$$
\leq|\mathcal{L}|^{-2} \sum_{x, y, l_{x}, l_{y}} p(x) p(y)\left(\operatorname{Tr}\left[\left(\mathbb{1}^{Z^{\prime}}-\Pi_{\hat{\mathcal{Z}}}^{Z^{\prime}}\right)\left(\rho^{\prime}\right)_{\left(x, l_{x}\right),\left(y, l_{y}\right), \delta}^{Z^{\prime}}\right]\right.
$$$$
\left.+\operatorname{Tr}\left[\left(\Pi^{\prime}\right)_{W_{\left(x, l_{x}\right),\left(y, l_{y}\right), \delta}^{Z^{\prime}}}^{Z^{\prime}}\left(\rho^{\prime}\right)_{\left(x, l_{x}\right),\left(y, l_{y}\right), \delta}^{Z^{\prime}}\right]\right)
$$

$$
\begin{array}{rl}
\leq & 4 \delta^{2} \\
+|\mathcal{L}|^{-2} \sum_{x, y, l_{x}, l_{y}} p & p(x) p(y)\left(\operatorname { T r } \left[\left(\mathbb{1}^{Z^{\prime}}-\Pi_{\hat{Z}^{\prime}}^{Z^{\prime}}\right)\left(\rho_{x, y}^{Z} \otimes|0\rangle\left\langle\left. 0\right|^{\mathbb{C}^{2}}\right)\right]\right.\right. \\
& +\operatorname{Tr}\left[\left(\Pi^{\prime}\right)_{\left.W_{\left(x, l_{x}\right),\left(y, l_{y}\right), \delta}^{Z^{\prime}}\left(\rho_{x, y}^{Z} \otimes|0\rangle\left\langle\left. 0\right|^{\mathbb{C}^{2}}\right)\right]\right)}^{Z}\right.
\end{array}
$$

$\leq \frac{6}{\delta^{2}}|\mathcal{L}|^{-2} \sum_{x, y, l_{x}, l_{y}} p(x) p(y)($ 


$$
\begin{aligned}
& \left(1-\operatorname{Tr}\left[\Pi_{X ; x, y}^{\hat{Z}}\left(\rho_{x, y}^{Z} \otimes|0\rangle\left\langle\left. 0\right|^{\mathbb{C}^{2}}\right)\right]\right)\right. \\
& +\left(1-\operatorname{Tr}\left[\Pi_{Y ; x, y}^{\hat{Z}}\left(\rho_{x, y}^{Z} \otimes|0\rangle\left\langle\left. 0\right|^{\mathbb{C}^{2}}\right)\right]\right)\right. \\
& \left.+\left(1-\operatorname{Tr}\left[\Pi_{x, y}^{\hat{Z}}\left(\rho_{x, y}^{Z} \otimes|0\rangle\langle 0| \mathbb{C}^{2}\right)\right]\right)\right) \\
& \begin{aligned}
& +4 \delta^{2} \\
\leq & \frac{6}{\delta^{2}}\left(\left(1-\operatorname{Tr}\left[\left(\Pi^{\prime \prime}\right)_{X}^{X Y Z} \rho^{X Y Z}\right]\right)+\left(1-\operatorname{Tr}\left[\left(\Pi^{\prime \prime}\right)_{Y}^{X Y Z} \rho^{X Y Z}\right]\right)\right. \\
& \left.+\left(1-\operatorname{Tr}\left[\left(\Pi^{\prime \prime}\right)^{X Y Z} \rho^{X Y Z}\right]\right)\right)+4 \delta^{2} \\
\leq & \frac{18 \epsilon}{\delta^{2}}+4 \delta^{2} .
\end{aligned}
\end{aligned}
$$

We choose $\delta:=\epsilon^{1 / 4}$ to get

$$
\operatorname{Tr}\left[\left(\mathbb{1}^{X^{\prime} Y^{\prime} Z^{\prime}}-\left(\Pi^{\prime}\right)^{X^{\prime} Y^{\prime} Z^{\prime}}\right)\left(\rho^{\prime}\right)^{X^{\prime} Y^{\prime} Z^{\prime}}\right] \leq 22 \epsilon^{1 / 2} .
$$

We now illustrate how the augmentation of $\mathcal{X}$ to $X^{\prime}$ and $y$ to $\mathcal{Y}^{\prime}$ helps in the so-called smoothing of the states

$$
\begin{aligned}
\left(\rho^{\prime}\right)_{\left(x, l_{x}\right), \delta}^{Z^{\prime}} & :=|\mathcal{L}|^{-1} \sum_{y, l_{y}} p(y)\left(\rho^{\prime}\right)_{\left(x, l_{x}\right),\left(y, l_{y}\right), \delta}^{Z^{\prime}}, \\
\left(\rho^{\prime}\right)_{\left(y, l_{y}\right), \delta}^{Z^{\prime}} & :=|\mathcal{L}|^{-1} \sum_{x, l_{x}} p(x)\left(\rho^{\prime}\right)_{\left(x, l_{x}\right),\left(y, l_{y}\right), \delta}^{Z^{\prime}}, \\
\left(\rho^{\prime}\right)_{\delta}^{Z^{\prime}} & :=|\mathcal{L}|^{-2} \sum_{x, l_{x}, y, l_{y}} p(x) p(y)\left(\rho^{\prime}\right)_{\left(x, l_{x}\right),\left(y, l_{y}\right), \delta}^{Z^{\prime}}
\end{aligned}
$$

As will become clear below, the tilting map $\mathcal{T}_{X Y ; l_{x}, l_{y}, \delta}$ is defined in such a way that $\left(\rho^{\prime}\right)_{\left(x, l_{x}\right), \delta}^{Z^{\prime}}$ is very close to $\mathcal{T}_{X ; l_{x}, \delta}\left(\rho_{x}^{Z} \otimes\right.$ $|0\rangle\left\langle\left. 0\right|^{\mathbb{C}^{2}}\right)$ in the $\ell_{\infty}$-norm, $\left(\rho^{\prime}\right)_{\left(y, l_{y}\right), \delta}^{Z^{\prime}, \delta}$ is very close to $\mathcal{T}_{Y ; l_{y}, \delta}\left(\rho_{y}^{Z} \otimes\right.$ $\left.|0\rangle\langle 0| \mathbb{C}^{2}\right)$ in the $\ell_{\infty}$-norm, and $\left(\rho^{\prime}\right)_{\delta}^{Z^{\prime}}$ is very close to $\rho^{Z} \otimes$ $|0\rangle\left\langle 0||^{\mathbb{C}^{2}}\right.$ in the $\ell_{\infty}$-norm. This closeness is what we mean by smoothing, and augmentation is required to ensure proper smoothing. Notice now that

$$
\begin{aligned}
\left(\rho^{\prime}\right)^{Y^{\prime}} \otimes & \left(\rho^{\prime}\right)^{X^{\prime} Z^{\prime}} \\
= & \left(|\mathcal{L}|^{-1} \sum_{y, l_{y}} p(y)\left|y, l_{y}\right\rangle\left\langle y, l_{y}\right|\right) \\
& \otimes\left(|\mathcal{L}|^{-1} \sum_{x, l_{x}} p(x)\left|x, l_{x}\right\rangle\left\langle x, l_{x}\right| \otimes\left(\rho^{\prime}\right)_{\left(x, l_{x}\right), \delta}^{Z^{\prime}}\right), \\
\left(\rho^{\prime}\right)^{X^{\prime}} \otimes\left(\rho^{\prime}\right)^{Y^{\prime} Z^{\prime}} & \left(|\mathcal{L}|^{-1} \sum_{x, l_{x}} p(x)\left|x, l_{x}\right\rangle\left\langle x, l_{x}\right|\right) \\
& \otimes\left(|\mathcal{L}|^{-1} \sum_{y, l_{y}} p(y)\left|y, l_{y}\right\rangle\left\langle y, l_{y}\right| \otimes\left(\rho^{\prime}\right)_{\left(y, l_{y}\right), \delta}^{Z^{\prime}}\right), \\
\left(\rho^{\prime}\right)^{X^{\prime} Y^{\prime}} \otimes\left(\rho^{\prime}\right)^{Y^{\prime} Z^{\prime}} & \left(|\mathcal{L}|^{-2} \sum_{x, l_{x}, y, l_{y}} p(x) p(y)\left|x, l_{x}, y, l_{y}\right\rangle\left\langle x, l_{x}, y, l_{y}\right|\right) \otimes\left(\rho^{\prime}\right)_{\delta}^{Z^{\prime}} .
\end{aligned}
$$

Observe that

$$
|\mathcal{L}|^{-1} \sum_{l_{y}} \mathcal{T}_{X Y ; l_{x}, l_{y}}(|h\rangle\langle h|)
$$

$$
\begin{aligned}
=\frac{|\mathcal{L}|^{-1}}{1+} & 2 \delta^{2} \\
& \sum_{l_{y}}( \\
& \left(|h\rangle+\delta|h\rangle\left|l_{x}\right\rangle\right)\left(\langle h|+\delta\langle h|\left\langle l_{x}\right|\right) \\
& +\delta\left(|h\rangle+\delta|h\rangle\left|l_{x}\right\rangle\right)\langle h|\left\langle l_{y}\right| \\
& +\delta|h\rangle\left|l_{y}\right\rangle\left(\langle h|+\delta\langle h|\left\langle l_{x}\right|\right) \\
& \left.+\delta^{2}|h\rangle\left\langle h|| l_{y}\right\rangle\left\langle l_{y}\right|\right) \\
=\frac{1+}{1+} & \delta^{2} \\
\hline & \mathcal{T}_{X ; l_{x}, \delta}(|h\rangle\langle h|)+N_{X ; l_{x}, \delta}(|h\rangle\langle h|),
\end{aligned}
$$

where

$$
\begin{aligned}
& N_{X ; l_{x}, \delta}(|h\rangle\langle h|) \\
& :=\frac{|\mathcal{L}|^{-1}}{1+2 \delta^{2}} \sum_{l_{y}}( \\
& \quad \delta\left(|h\rangle+\delta|h\rangle\left|l_{x}\right\rangle\right)\langle h|\left\langle l_{y}\right| \\
& +\delta|h\rangle\left|l_{y}\right\rangle\left(\langle h|+\delta\langle h|\left\langle l_{x}\right|\right) \\
& \left.+\delta^{2}|h\rangle\left\langle h \| l_{y}\right\rangle\left\langle l_{y}\right|\right) .
\end{aligned}
$$

The vectors $\left|l_{y}\right\rangle$ are orthogonal as $l_{y}$ runs through the computational basis vectors of $\mathcal{L}^{Y}$. From this, it is easy to see that

$$
\begin{gathered}
\left\|N_{X ; l_{x}, \delta}(|h\rangle\langle h|)\right\|_{\infty} \\
\leq \frac{|\mathcal{L}|^{-1}}{1+2 \delta^{2}}\left(\| \sum_{l_{y}} \delta\left(|h\rangle+\delta|h\rangle\left|l_{x}\right\rangle\right)\langle h|\left\langle l_{y}\right| \|_{\infty}\right. \\
+\| \sum_{l_{y}} \delta|h\rangle\left|l_{y}\right\rangle\left(\langle h|+\delta\langle h|\left\langle l_{x}\right|\right) \|_{\infty} \\
\left.+\| \sum_{l_{y}} \delta^{2}|h\rangle\left\langle h \mid \| l_{y}\right\rangle\left\langle l_{y}\right| \|_{\infty}\right) \\
=\frac{|\mathcal{L}|^{-1}}{1+2 \delta^{2}}\left(\delta|\| h\rangle+\delta|h\rangle\left|l_{x}\right\rangle\left\|_{2} \cdot\right\| \sum_{l_{y}}\left|l_{y}\right\rangle\left\|_{2}\right\| \|_{l_{y}} \mid l_{\left.y_{y}\right\rangle}+\delta^{2}\right) \\
\left.\leq \frac{3 \delta}{\sqrt{|\mathcal{L}|}} . \delta \| h\right\rangle+\delta|h\rangle\left|l_{x}\right\rangle\left\|_{2} \cdot\right\| \sum_{2}
\end{gathered}
$$

Similarly, one can define

$$
\begin{aligned}
& N_{Y ; l_{y}, \delta}(|h\rangle\langle h|) \quad:=\frac{|\mathcal{L}|^{-1}}{1+2 \delta^{2}} \sum_{l_{x}}\left(\delta\left(|h\rangle+\delta|h\rangle\left|l_{y}\right\rangle\right)\langle h|\left\langle l_{x}\right|\right. \\
& +\delta|h\rangle\left|l_{x}\right\rangle\left(\langle h|+\delta\langle h|\left\langle l_{y}\right|\right) \\
& \left.+\delta^{2}|h\rangle\left\langle h|| l_{x}\right\rangle\left\langle l_{x}\right|\right) \text {, } \\
& N_{\delta}(|h\rangle\langle h|):=\frac{|\mathcal{L}|^{-2}}{1+2 \delta^{2}} \sum_{l_{x}, l_{y}}\left(\delta|h\rangle\left(\langle h|\left\langle l_{x}\right|+\langle h|\left\langle l_{y}\right|\right)\right. \\
& +\delta\left(|h\rangle\left|l_{x}\right\rangle+|h\rangle\left|l_{y}\right\rangle\right)\langle h| \\
& +\delta^{2}|h\rangle\left\langle h\left|\left(\left|l_{x}\right\rangle+\left|l_{y}\right\rangle\right)\left(\left\langle l_{x}\right|+\left\langle l_{y}\right|\right)\right),\right.
\end{aligned}
$$


and show that their $\ell_{\infty}$-norms are upper bounded by $\frac{3 \delta}{\sqrt{|\mathcal{L}|}}$ each. We can also extend the maps $N_{X ; l_{x}, \delta}, N_{Y ; l_{y}, \delta}, N_{\delta}$ to mixed states in the natural manner.

We can thus write

$$
\begin{aligned}
\left(\rho^{\prime}\right)_{\left(x, l_{x}\right), \delta}^{Z^{\prime}}= & \frac{1+\delta^{2}}{1+2 \delta^{2}} \mathcal{T}_{X ; l_{x}, \delta}\left(\rho_{x}^{Z} \otimes|0\rangle\left\langle\left. 0\right|^{\mathbb{C}^{2}}\right)\right. \\
& +N_{X ; l_{x}, \delta}\left(\rho_{x}^{Z} \otimes|0\rangle\left\langle\left. 0\right|^{\mathbb{C}^{2}}\right),\right. \\
\left(\rho^{\prime}\right)_{\left(y, l_{y}\right), \delta}^{Z^{\prime}}= & \frac{1+\delta^{2}}{1+2 \delta^{2}} \mathcal{T}_{Y ; l_{y}, \delta}\left(\rho _ { y } ^ { Z } \otimes | 0 \rangle \left\langle\left.0\right|^{\left.\mathbb{C}^{2}\right)}\right.\right. \\
& +N_{Y ; l_{y}, \delta}\left(\rho_{y}^{Z} \otimes|0\rangle\left\langle\left. 0\right|^{\mathbb{C}^{2}}\right),\right. \\
\left(\rho^{\prime}\right)_{\delta}^{Z^{\prime}}= & \frac{1}{1+2 \delta^{2}}\left(\rho^{Z} \otimes|0\rangle\left\langle\left. 0\right|^{\mathbb{C}^{2}}\right)\right. \\
& +N_{\delta}\left(\rho ^ { Z } \otimes | 0 \rangle \left\langle\left.0\right|^{\left.\mathbb{C}^{2}\right),}\right.\right.
\end{aligned}
$$

with

$$
\begin{aligned}
\left\|N _ { X ; l _ { x } , \delta } \left(\rho_{x}^{Z} \otimes|0\rangle\left\langle\left. 0\right|^{\mathbb{C}^{2}}\right) \|_{\infty}\right.\right. & \leq \frac{3 \delta}{\sqrt{|\mathcal{L}|}}, \\
\left\|N _ { Y ; l _ { y } , \delta } \left(\rho_{y}^{Z} \otimes|0\rangle\left\langle\left. 0\right|^{\mathbb{C}^{2}}\right) \|_{\infty}\right.\right. & \leq \frac{3 \delta}{\sqrt{|\mathcal{L}|}}, \\
\left\|N _ { \delta } \left(\rho^{Z} \otimes|0\rangle\left\langle\left. 0\right|^{\mathbb{C}^{2}}\right) \|_{\infty}\right.\right. & \leq \frac{3 \delta}{\sqrt{|\mathcal{L}|}} .
\end{aligned}
$$

Observe now that

$$
\begin{aligned}
\left\|\left(\Pi^{\prime}\right)_{\left(x, l_{x}\right),\left(y, l_{y}\right), \delta}^{Z^{\prime}}\right\|_{1} & \leq\left\|\left(\mathbb{1}^{Z^{\prime}}-\left(\Pi^{\prime}\right)_{W_{\left(x, l_{x}\right),\left(y, l_{y}\right), \delta}^{Z^{\prime}}}^{\prime}\right)\right\|_{\infty}^{2}\left\|\Pi_{\hat{\mathcal{Z}}}^{Z^{\prime}}\right\|_{1} \\
& =2|\mathcal{Z}| .
\end{aligned}
$$

Hence, we get the upper bound

$$
\begin{aligned}
& \operatorname{Tr}\left[\left(\Pi^{\prime}\right)_{\left(x, l_{x}\right),\left(y, l_{y}\right), \delta}^{Z^{\prime}}\left(\rho^{\prime}\right)_{\left(x, l_{x}\right), \delta}^{Z^{\prime}}\right] \\
& \leq \operatorname{Tr}\left[\left(\Pi^{\prime}\right)_{\left(x, l_{x}\right),\left(y, l_{y}\right), \delta}^{Z^{\prime}}\left(\mathcal{T}_{X ; l_{x}, \delta}\left(\rho_{x}^{Z} \otimes|0\rangle\left\langle\left. 0\right|^{\mathbb{C}^{2}}\right)\right)\right]\right. \\
& +\left\|\left(\Pi^{\prime}\right)_{\left(x, l_{x}\right),\left(y, l_{y}\right), \delta}^{Z^{\prime}}\right\|_{1} \cdot\left\|N _ { X ; l _ { x } , \delta } \left(\rho_{x}^{Z} \otimes|0\rangle\left\langle\left. 0\right|^{\mathbb{C}^{2}}\right) \|_{\infty}\right.\right. \\
& \leq \operatorname{Tr}\left[\left(\mathbb{1}^{\mathcal{Z}^{\prime}}-\left(\Pi^{\prime}\right)_{W_{\left(x, l_{x}\right),\left(, l_{y}\right), \delta}^{Z^{\prime}}}\right)\left(\mathcal{T}_{X ; l_{x}, \delta}\left(\rho_{x}^{Z} \otimes|0\rangle\left\langle\left. 0\right|^{\mathbb{C}^{2}}\right)\right)\right]\right. \\
& +\frac{6 \delta|\mathcal{Z}|}{\sqrt{|\mathcal{L}|}} \\
& \leq \operatorname{Tr}\left[\left(\mathbb{1}^{\mathcal{Z}^{\prime}}-\left(\Pi^{\prime}\right)_{W_{X ;\left(x, l_{x}\right),\left(y, l_{y}\right), \delta}^{Z^{\prime}}}^{\prime}\right)\left(\mathcal{T}_{X ; l_{x}, \delta}\left(\rho_{x}^{Z} \otimes|0\rangle\left\langle\left. 0\right|^{\mathbb{C}^{2}}\right)\right)\right]\right. \\
& +\frac{6 \delta|\mathcal{Z}|}{\sqrt{|\mathcal{L}|}}
\end{aligned}
$$

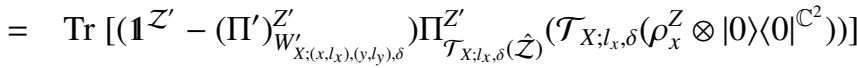

$$
\begin{aligned}
& +\frac{6 \delta|\mathcal{Z}|}{\sqrt{|\mathcal{L}|}} \\
& =\operatorname{Tr}\left[\left(\mathbb{1}^{\mathcal{T}_{X ; l_{x}, \delta}(\hat{\mathcal{Z}})}-\left(\Pi^{\prime}\right)_{W_{X ;(x, l)}^{\prime} \mathcal{T}_{\left.X, l_{x}, l_{y}\right), \delta}(\hat{\mathcal{Z}})}^{\mathcal{F}^{\prime}}\right)\left(\mathcal{T}_{X ; l_{x}, \delta}\left(\rho_{x}^{Z} \otimes|0\rangle\left\langle\left. 0\right|^{\mathbb{C}^{2}}\right)\right)\right]\right. \\
& +\frac{6 \delta|\mathcal{Z}|}{\sqrt{|\mathcal{L}|}} \\
& =\operatorname{Tr}\left[\left(\mathbb{1}^{\hat{\mathcal{Z}}}-\Pi_{W_{X ; x, y}}^{\hat{\mathcal{Z}}}\right)\left(\rho_{x}^{Z} \otimes|0\rangle\left\langle\left. 0\right|^{\mathbb{C}^{2}}\right)\right]+\frac{6 \delta|\mathcal{Z}|}{\sqrt{|\mathcal{L}|}}\right.
\end{aligned}
$$

$$
\begin{aligned}
& =\operatorname{Tr}\left[\Pi_{X ; x, y}^{\hat{Z}}\left(\rho_{x}^{Z} \otimes|0\rangle\left\langle\left. 0\right|^{\mathbb{C}^{2}}\right)\right]+\frac{6 \delta|\mathcal{Z}|}{\sqrt{|\mathcal{L}|}}\right. \\
& =\operatorname{Tr}\left[\left(\Pi^{\prime \prime}\right)_{X ; x, y}^{Z} \rho_{x}^{Z}\right]+\frac{6 \delta|\mathcal{Z}|}{\sqrt{|\mathcal{L}|}} .
\end{aligned}
$$

Above, we used the facts that $W_{X ;\left(x, l_{x}\right),\left(y, l_{y}\right), \delta}^{\prime} \leq W_{\left(x, l_{x}\right),\left(y, l_{y}\right), \delta}^{\prime}$, $W_{X ;\left(x, l_{x}\right),\left(y, l_{y}\right), \delta}^{\prime} \leq \mathcal{T}_{X ; l_{x}, \delta}(\hat{\mathcal{Z}})$, and that $\mathcal{T}_{X ; l_{x}, \delta}$ is an isometry. Using Equation 6, we now get

$$
\begin{aligned}
\operatorname{Tr} & {\left[\left(\Pi^{\prime}\right)^{X^{\prime} Y^{\prime} Z^{\prime}}\left(\left(\rho^{\prime}\right)^{Y^{\prime}} \otimes\left(\rho^{\prime}\right)^{X^{\prime} Z^{\prime}}\right)\right] } \\
& =|\mathcal{L}|^{-2} \sum_{y, l_{y}} p(y) \sum_{x, l_{x}} p(x) \operatorname{Tr}\left[\left(\Pi^{\prime}\right)_{\left(x, l_{x}\right),\left(y, l_{y}\right), \delta}^{Z^{\prime}}\left(\rho^{\prime}\right)_{\left(x, l_{x}\right), \delta}^{Z^{\prime}}\right] \\
& \leq|\mathcal{L}|^{-2} \sum_{y, l_{y}} p(y) \sum_{x, l_{x}} p(x) \operatorname{Tr}\left[\left(\Pi^{\prime \prime}\right)_{X ; x, y}^{Z} \rho_{x}^{Z}\right]+\frac{6 \delta|\mathcal{Z}|}{\sqrt{|\mathcal{L}|}} \\
& =\sum_{x, y} p(x) p(y) \operatorname{Tr}\left[\left(\Pi^{\prime \prime}\right)_{X ; x, y}^{Z} \rho_{x}^{Z}\right]+\frac{6 \delta|\mathcal{Z}|}{\sqrt{|\mathcal{L}|}} \\
& =\operatorname{Tr}\left[\left(\Pi^{\prime \prime}\right)_{X}^{X Y Z}\left(\rho^{Y} \otimes \rho^{X Z}\right)\right]+\frac{6 \delta|\mathcal{Z}|}{\sqrt{|\mathcal{L}|}} \\
& \leq 2^{-I_{H}^{\epsilon}(Y: X Z)}+\frac{6 \delta|\mathcal{Z}|}{\sqrt{|\mathcal{L}|}} .
\end{aligned}
$$

We choose $|\mathcal{L}|$ large enough so that the second term in the last inequality is less than $\min \left\{2^{-I_{H}^{\epsilon}(Y: X Z)}, 2^{-I_{H}^{\epsilon}(X: Y Z)}, 2^{-I_{H}^{\epsilon}(X Y: Z)}\right\}$. Thus, we have the inequalities

$$
\begin{aligned}
& \operatorname{Tr}\left[\left(\Pi^{\prime}\right)^{X^{\prime} Y^{\prime} Z^{\prime}}\left(\left(\rho^{\prime}\right)^{Y^{\prime}} \otimes\left(\rho^{\prime}\right)^{X^{\prime} Z^{\prime}}\right)\right] \leq 2^{-I_{H}^{\epsilon}(Y: X Z)+1}, \\
& \operatorname{Tr}\left[\left(\Pi^{\prime}\right)^{X^{\prime} Y^{\prime} Z^{\prime}}\left(\left(\rho^{\prime}\right)^{X^{\prime}} \otimes\left(\rho^{\prime}\right)^{Y^{\prime} Z^{\prime}}\right)\right] \leq 2^{-I_{H}^{\epsilon}(X: Y Z)+1}, \\
& \operatorname{Tr}\left[\left(\Pi^{\prime}\right)^{X^{\prime} Y^{\prime} Z^{\prime}}\left(\left(\rho^{\prime}\right)^{X^{\prime} Y^{\prime}} \otimes\left(\rho^{\prime}\right)^{Z^{\prime}}\right)\right] \leq 2^{-I_{H}^{\epsilon}(X Y: Z)+1} .
\end{aligned}
$$

We now describe the decoding strategy that Charlie follows in order to try and guess the message pair $\left(m_{1}, m_{2}\right)$ that was actually sent, given the output of $\mathfrak{C}^{\prime \prime}$. Charlie uses the pretty good measurement [12, 13, 6, 7] constructed from the POVM elements $\left(\Pi^{\prime}\right)_{\left(x, l_{x}\right)\left(m_{1}\right),\left(y, l_{y}\right)\left(m_{2}\right), \delta}^{Z^{\prime}}$, where $\left(m_{1}, m_{2}\right) \times$ $\left[2^{R_{1}}\right] \times\left[2^{R_{2}}\right]$. We now analyse the expectation, under the choice of a random codebook $C$, of the error probability of Charlie's decoding algorithm. Suppose the message pair $\left(m_{1}, m_{2}\right)$ is inputted to $\mathfrak{C}^{\prime \prime}$. The output of $\mathfrak{C}^{\prime \prime}$ is the state $\left(\rho^{\prime}\right)_{\left(x, l_{x}\right)\left(m_{1}\right),\left(y, l_{y}\right)\left(m_{2}\right), \delta^{\prime}}^{Z^{\prime}}$ Let $\Lambda_{\hat{m}_{1}, \hat{m}_{2}}^{Z^{\prime}}$ be the POVM element corresponding to decoded output $\left(\hat{m}_{1}, \hat{m}_{2}\right)$ arising from the pretty good measurement. By the Hayashi-Nagaoka inequality [14], the decoding error for $\left(m_{1}, m_{2}\right)$ is upper bounded by

$$
\begin{aligned}
& \operatorname{Tr}\left[\left(\mathbb{1}^{Z^{\prime}}-\Lambda_{m_{1}, m_{2}}^{Z^{\prime}}\right)\left(\rho^{\prime}\right)_{\left(x, l_{x}\right)\left(m_{1}\right),\left(y, l_{y}\right)\left(m_{2}\right), \delta}^{Z^{\prime}}\right] \\
& \leq 2 \operatorname{Tr}\left[\left(\mathbb{1}^{Z^{\prime}}-\left(\Pi^{\prime}\right)_{\left(x, l_{x}\right)\left(m_{1}\right),\left(y, l_{y}\right)\left(m_{2}\right), \delta}^{Z^{\prime}}\right)\right. \\
& \left.+4 \sum_{\left(\hat{m}_{1}, \hat{m}_{2}\right) \neq\left(m_{1}, m_{2}\right)}^{Z^{\prime}}\right)_{\left(x, l_{x}\right)\left(m_{1}\right),\left(y, l_{y}\right)\left(m_{2}\right), \delta} \operatorname{Tr}\left[\left(\Pi^{\prime}\right)_{\left(x, l_{x}\right)\left(\hat{m}_{1}\right),\left(y, l_{y}\right)\left(\hat{m}_{2}\right), \delta}^{Z^{\prime}}\left(\rho^{\prime}\right)_{\left(x, l_{x}\right)\left(m_{1}\right),\left(y, l_{y}\right)\left(m_{2}\right), \delta}^{Z^{\prime}}\right] \\
& =2 \operatorname{Tr}\left[\left(\mathbb{1}^{Z^{\prime}}-\left(\Pi^{\prime}\right)_{\left(x, l_{x}\right)\left(m_{1}\right),\left(y, l_{y}\right)\left(m_{2}\right), \delta}^{Z^{\prime}}\right)\right. \\
& \left.\left(\rho^{\prime}\right)_{\left(x, l_{x}\right)\left(m_{1}\right),\left(y, l_{y}\right)\left(m_{2}\right), \delta}^{Z^{\prime}}\right] \\
& +4 \sum_{\hat{m}_{1} \neq m_{1}} \operatorname{Tr}\left[\left(\Pi^{\prime}\right)_{\left(x, l_{x}\right)\left(\hat{m}_{1}\right),\left(y, l_{y}\right)\left(m_{2}\right), \delta}^{Z^{\prime}}\right.
\end{aligned}
$$




$$
\begin{gathered}
\left.\left(\rho^{\prime}\right)_{\left(x, l_{x}\right)\left(m_{1}\right),\left(y, l_{y}\right)\left(m_{2}\right), \delta}^{Z^{\prime}}\right] \\
+4 \sum_{\hat{m}_{2} \neq m_{2}} \operatorname{Tr}\left[\left(\Pi^{\prime}\right)_{\left(x, l_{x}\right)\left(m_{1}\right),\left(y, l_{y}\right)\left(\hat{m}_{2}\right), \delta}^{Z^{\prime}}\right. \\
+4 \sum_{\hat{m}_{1} \neq m_{1}, \hat{m}_{2} \neq m_{2}} \operatorname{Tr}\left[\left(\rho^{\prime}\right)_{\left(x, l_{x}\right)\left(m_{1}\right),\left(y, l_{y}\right)\left(m_{2}\right), \delta}^{Z^{\prime}}\right)_{\left(x, l_{x}\right)\left(\hat{m}_{1}\right),\left(y, l_{y}\right)\left(\hat{m}_{2}\right), \delta}^{Z^{\prime}} \\
\left.\left(\rho^{\prime}\right)_{\left(x, l_{x}\right)\left(m_{1}\right),\left(y, l_{y}\right)\left(m_{2}\right), \delta}^{Z^{\prime}}\right] .
\end{gathered}
$$

The expectation, over the choice of the random codebook $C$, of the decoding error for $\left(m_{1}, m_{2}\right)$ is upper bounded by

$$
\begin{aligned}
& \underset{C}{\mathbf{E}}\left[\operatorname{Tr}\left[\left(\mathbb{1}^{Z^{\prime}}-\Lambda_{m_{1}, m_{2}}^{Z^{\prime}}\right)\left(\rho^{\prime}\right)_{\left(x, l_{x}\right)\left(m_{1}\right),\left(y, l_{y}\right)\left(m_{2}\right), \delta}^{Z^{\prime}}\right]\right] \\
& \leq 2 \underset{C}{\mathbf{E}}\left[\operatorname { T r } \left[\left(\mathbb{1}^{Z^{\prime}}-\left(\Pi^{\prime}\right)_{\left(x, l_{x}\right)\left(m_{1}\right),\left(y, l_{y}\right)\left(m_{2}\right), \delta}^{Z^{\prime}}\right)\right.\right. \\
& \left.\left.\left(\rho^{\prime}\right)_{\left(x, l_{x}\right)\left(m_{1}\right),\left(y, l_{y}\right)\left(m_{2}\right), \delta}^{Z^{\prime}}\right]\right] \\
& +4 \sum_{\hat{m}_{1} \neq m_{1}} \underset{C}{\mathbf{E}}\left[\operatorname { T r } \left[\left(\Pi^{\prime}\right)_{\left(x, l_{x}\right)\left(\hat{m}_{1}\right),\left(y, l_{y}\right)\left(m_{2}\right), \delta}^{Z^{\prime}}\right.\right. \\
& \left.\left.\left(\rho^{\prime}\right)_{\left(x, l_{x}\right)\left(m_{1}\right),\left(y, l_{y}\right)\left(m_{2}\right), \delta}^{Z^{\prime}}\right]\right] \\
& +4 \sum_{\hat{m}_{2} \neq m_{2}} \underset{C}{\mathbf{E}}\left[\operatorname { T r } \left[\left(\Pi^{\prime}\right)_{\left(x, l_{x}\right)\left(m_{1}\right),\left(y, l_{y}\right)\left(\hat{m}_{2}\right), \delta}^{Z^{\prime}}\right.\right. \\
& \left.\left.\left(\rho^{\prime}\right)_{\left(x, l_{x}\right)\left(m_{1}\right),\left(y, l_{y}\right)\left(m_{2}\right), \delta}^{Z^{\prime}}\right]\right] \\
& +4 \sum_{\hat{m}_{1} \neq m_{1}, \hat{m}_{2} \neq m_{2}} \underset{C}{\mathbf{E}}\left[\operatorname { T r } \left[\left(\Pi^{\prime}\right)_{\left(x, l_{x}\right)\left(\hat{m}_{1}\right),\left(y, l_{y}\right)\left(\hat{m}_{2}\right), \delta}^{Z^{\prime}}\right.\right. \\
& \left.\left.\left(\rho^{\prime}\right)_{\left(x, l_{x}\right)\left(m_{1}\right),\left(y, l_{y}\right)\left(m_{2}\right), \delta}^{Z^{\prime}}\right]\right] \\
& =2|\mathcal{L}|^{-3} \sum_{x, y, l_{x}, l_{y}} p(x) p(y) \\
& \operatorname{Tr}\left[\left(\mathbb{1}^{Z^{\prime}}-\left(\Pi^{\prime}\right)_{\left(x, l_{x}\right),\left(y, l_{y}\right), \delta}^{Z^{\prime}}\right)\right. \\
& \left.\left(\rho^{\prime}\right)_{\left(x, l_{x}\right),\left(y, l_{y}\right), \delta}^{Z^{\prime}}\right] \\
& +4\left(2^{R_{1}}-1\right)|\mathcal{L}|^{-4} \sum_{x, l_{x}, x^{\prime}, l_{x}^{\prime}, y, l_{y}} p(x) p\left(x^{\prime}\right) p(y) \\
& \left.\operatorname{Tr}\left[\left(\Pi^{\prime}\right)_{\left(x^{\prime}, l_{x}^{\prime}\right),\left(y, l_{y}\right), \delta}^{Z^{\prime}}\right)\left(\rho^{\prime}\right)_{\left(x, l_{x}\right),\left(y, l_{y}\right), \delta}^{Z^{\prime}}\right] \\
& +4\left(2^{R_{2}}-1\right)|\mathcal{L}|^{-4} \sum_{x, l_{x}, x^{\prime}, l_{x}^{\prime}, y, l_{y}} p(x) p(y) p\left(y^{\prime}\right) \\
& \left.\operatorname{Tr}\left[\left(\Pi^{\prime}\right)_{\left(x, l_{x}\right),\left(y^{\prime}, l_{y}^{\prime}\right), \delta}^{Z^{\prime}}\right)\left(\rho^{\prime}\right)_{\left(x, l_{x}\right),\left(y, l_{y}\right), \delta}^{Z^{\prime}}\right] \\
& +4\left(2^{R_{1}}-1\right)\left(2^{R_{2}}-1\right)|\mathcal{L}|^{-5} \\
& \sum_{x, l_{x}, x^{\prime}, l_{x}^{\prime}, y, l_{y}, y^{\prime}, l_{y}^{\prime}} p(x) p\left(x^{\prime}\right) p(y) p\left(y^{\prime}\right) \\
& \left.\operatorname{Tr}\left[\left(\Pi^{\prime}\right)_{\left(x^{\prime}, l_{x}^{\prime}\right),\left(y^{\prime}, l_{y}^{\prime}\right), \delta}^{Z^{\prime}}\right)\left(\rho^{\prime}\right)_{\left(x, l_{x}\right),\left(y, l_{y}\right), \delta}^{Z^{\prime}}\right] \\
& =2 \operatorname{Tr}\left[\left(\mathbb{1}^{X^{\prime} Y^{\prime} Z^{\prime}}-\left(\Pi^{\prime}\right)^{X^{\prime} Y^{\prime} Z^{\prime}}\right)\left(\rho^{\prime}\right)^{X^{\prime} Y^{\prime} Z^{\prime}}\right] \\
& +4\left(2^{R_{1}}-1\right) \operatorname{Tr}\left[\left(\Pi^{\prime}\right)^{X^{\prime} Y^{\prime} Z^{\prime}}\left(\left(\rho^{\prime}\right)^{X^{\prime}} \otimes\left(\rho^{\prime}\right)^{Y^{\prime} Z^{\prime}}\right)\right] \\
& +4\left(2^{R_{2}}-1\right) \operatorname{Tr}\left[\left(\Pi^{\prime}\right)^{X^{\prime} Y^{\prime} Z^{\prime}}\left(\left(\rho^{\prime}\right)^{Y^{\prime}} \otimes\left(\rho^{\prime}\right)^{X^{\prime} Z^{\prime}}\right)\right] \\
& +4\left(2^{R_{1}}-1\right)\left(2^{R_{2}}-1\right) \operatorname{Tr}\left[\left(\Pi^{\prime}\right)^{X^{\prime} Y^{\prime} Z^{\prime}}\left(\left(\rho^{\prime}\right)^{X^{\prime} Y^{\prime}} \otimes\left(\rho^{\prime}\right)^{Z^{\prime}}\right)\right] \\
& \leq 44 \epsilon^{1 / 2}+2^{R_{1}+1-I_{H}^{\epsilon}(X: Y Z)_{\rho}}+2^{R_{2}+1-I_{H}^{\epsilon}(Y: X Z)_{\rho}} \\
& +2^{R_{1}+R_{2}+1-I_{H}^{\epsilon}(X Y: Z)_{\rho}},
\end{aligned}
$$

where we used Equations 7,8 in the last inequality above.
Choosing a rate pair $\left(R_{1}, R_{2}\right)$ satisfying

$$
\begin{aligned}
R_{1} & \leq I_{H}^{\epsilon}(X: Y Z)_{\rho}-1-\log \frac{1}{\epsilon}, \\
R_{2} & \leq I_{H}^{\epsilon}(Y: X Z)_{\rho}-1-\log \frac{1}{\epsilon}, \\
R_{1}+R_{2} & \leq I_{H}^{\epsilon}(X Y: Z)_{\rho}-1-\log \frac{1}{\epsilon},
\end{aligned}
$$

ensures that the expected average decoding error for channel $\mathfrak{C}^{\prime \prime}$ is at most $47 \epsilon^{1 / 2}$. This implies that the expected average decoding error for the original channel $\mathbb{C}$ is at most $49 \epsilon^{1 / 2}$. Thus there exists a codebook $C$ with average decoding error for $\mathfrak{C}$ at most $49 \epsilon^{1 / 2}$. By a standard technique of taking maps from classical symbols to arbitrary quantum states, we can then prove the following theorem.

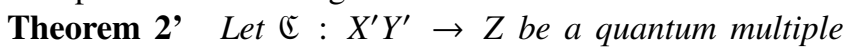
access channel. Let $\mathcal{X}, \mathcal{Y}$ be two new sample spaces. For every element $x \in \mathcal{X}$, let $\sigma_{x}^{X^{\prime}}$ be a quantum state in the input Hilbert space $X^{\prime}$ of $\mathfrak{C}$. Similarly, for every element $y \in \mathcal{Y}$, let $\sigma_{y}^{Y^{\prime}}$ be a quantum state in the input Hilbert space $Y^{\prime}$ of $\mathfrak{C}$. Let $p(x) p(y)$ be a probability distribution on $\mathcal{X} \times \mathcal{Y}$. Consider the classical quantum state

$$
\rho^{X Y Z}:=\sum_{x, y} p(x) p(y)|x, y\rangle\left\langle x,\left.y\right|^{X Y} \otimes\left(\mathbb{C}\left(\sigma_{x}^{X^{\prime}} \otimes \sigma_{y}^{Y^{\prime}}\right)\right)^{Z} .\right.
$$

Let $R_{1}, R_{2}, \epsilon$, be such that

$$
\begin{aligned}
R_{1} & \leq I_{H}^{\epsilon}(X: Y Z)_{\rho}-1-\log \frac{1}{\epsilon}, \\
R_{2} & \leq I_{H}^{\epsilon}(Y: X Z)_{\rho}-1-\log \frac{1}{\epsilon}, \\
R_{1}+R_{2} & \leq I_{H}^{\epsilon}(X Y: Z)_{\rho}-1-\log \frac{1}{\epsilon} .
\end{aligned}
$$

Then there exists an $\left(R_{1}, R_{2}, 49 \epsilon^{1 / 2}\right)$-quantum MAC code for sending classical information through $\mathfrak{C}$.

\section{The one-shot classical quantum joint typicality lemma}

In this section, we state various versions of our one-shot classical quantum joint typicality lemmas. We first state the 'intersection case' in Lemma 1, where we only have to take a socalled 'intersection' of POVM elements. This can be viewed as the classical quantum version of Fact 1 when $t=1$ i.e. when only intersection of classical POVM elements needs to be taken in Fact 1 , not the union. In fact many channel coding applications in quantum Shannon theory, e.g. the 'pentagonal' inner bound for the cq-MAC in this paper, use classical quantum states with only one quantum register where only 'intersection' of POVM elements needs to be taken. For these applications, we state an even simpler joint typicality lemma in Corollary 4. Nevertheless there are applications that require 'interesection' POVM elements acting on classical quantum states with more than one quantum register e.g. Marton's inner bound with common message for sending classical information over an entanglement assisted quantum broadcast channel [11]. These applications require the extra 
strength of Lemma 1. For comprehensiveness, in the special case where there is no classical system i.e. $c=0$, we get a simplification of Lemma 1 which we state in Corollary 3

After stating and proving Lemma 1 and Corollaries 3 . 4. we state and prove the one-shot classical quantum conditional joint typicality lemma, general case in Theorem 1 . Its statement takes care of the setting when one has to take a 'union of intersection' of POVM elements. This can be viewed as the classical quantum version of Fact 1 . We call this statement as the general case because it is the strongest statement that can be proved with the techniques developed in this paper. Even though most channel coding applications use tensor products of bipartitions as the negative hypotheses, we nevertheless state our general case with tensor products of arbitrary subpartitions as the negative hypotheses. This is for generality as well as keeping an eye on potential applications like generalised quantum Slepian-Wolf [28] where the negative hypothesis is a tensor product of three marginals, though the entropic quantity in Slepian-Wolf is of the covering type where our joint typicality lemmas do not seem to apply. Many applications, e.g. the Chong-MotaniGarg-El Gamal inner bound for sending classical information over a classical quantum interference channel [11], use classical quantum states with only one quantum register but involve an 'union of intersection' of POVM elements. For these applications, we state a simpler version of Theorem 1 in Corollary 5

The essential part of the proofs of all the joint typicality results obtained in this paper is encapsulated into a technical statement in Proposition 4, which should be of independent interest. Its proof is deferred to Appendix I.

We now state our one-shot classical quantum joint typicality lemma, intersection case.

Lemma 1 (cq joint typicality lem., intersec. case) Let $\mathcal{H}, \mathcal{L}$ be Hilbert spaces and $\mathcal{X}$ be a finite set. We will also use $\mathcal{X}$ to denote the Hilbert space with computational basis elements indexed by the set $\mathcal{X}$. Let $c$ be a non-negative and $k$ a positive integer. Let $A_{1} \cdots A_{k}$ be a k-partite system where each $A_{i}$ is isomorphic to $\mathcal{H}$. For every $\mathbf{x} \in \mathcal{X}^{c}$, let $\rho_{\mathbf{x}}$ be a quantum state in $A_{[k]}$. Consider the augmented $k$-partite system $A_{1}^{\prime} \cdots A_{k}^{\prime}$ where each $A_{i}^{\prime} \cong A_{i}^{\prime \prime} \otimes \mathcal{L}$, and each $A_{i}^{\prime \prime}$ is defined as

$$
A_{i}^{\prime \prime}:=\left(\mathcal{H} \otimes \mathbb{C}^{2}\right) \oplus \bigoplus_{S: i \in S \subseteq[c] \cup[k]}\left(\mathcal{H} \otimes \mathbb{C}^{2}\right) \otimes \mathcal{L}^{\otimes|S|} .
$$

Also define $\mathcal{X}^{\prime}:=\mathcal{X} \otimes \mathcal{L}$.

Below, $\mathbf{x}, \mathbf{l}$ denote computational basis vectors of $X^{[c]}$, $\mathcal{L}^{\otimes([c] \cup[k])}$. Let $p(\cdot)$ be a probability distribution on the vectors x. Define the classical quantum state

$$
\rho^{\mathcal{X}_{[c]} A_{[k]}}:=\sum_{\mathbf{x}} p(\mathbf{x})|\mathbf{x}\rangle\left\langle\left.\mathbf{x}\right|^{X_{[c]}} \otimes \rho_{\mathbf{x}}^{A_{[k]}} .\right.
$$

Let $\frac{\boldsymbol{1}^{\mathcal{L}^{\otimes(c+k)}}}{\mid \mathcal{L}^{c+k}}$ denote the completely mixed state on $(c+k)$ tensor copies of $\mathcal{L}$. View $\rho_{\mathbf{x}}^{A_{[k]}} \otimes(|0\rangle\langle 0|)^{\left(\mathbb{C}^{2}\right)^{\otimes k}} \otimes \frac{\mathbb{1}^{\mathcal{L}^{8(c+k)}}}{|\mathcal{L}|^{[+k}}$ as a state in $A_{[k]}^{\prime}$ under the natural embedding viz. the embedding in the ith system is into the first summand of $A_{i}^{\prime \prime}$ defined above tensored with $\mathcal{L}$. Similarly, view $\rho^{\mathcal{X}_{[c]} A_{[k]}} \otimes(|0\rangle\langle 0|)^{\left(\mathbb{C}^{2}\right)^{\otimes k}} \otimes \frac{\mathbb{1}^{\mathcal{L}^{\otimes(c+k)}}}{\mid \mathcal{L}^{c+k}}$ as a state in $X_{[c]}^{\prime} A_{[k]}^{\prime}$ under the natural embedding.

Let $0 \leq \delta \leq 1$. For each pseudosubpartition $\left(S_{1}, \ldots, S_{l}\right)+\vdash$ $[c] \cup[k]$, let $0 \leq \epsilon_{\left(S_{1}, \ldots, S_{l}\right)} \leq 1$. Then, there is a state $\rho^{\prime}$ and $a$ POVM element $\Pi^{\prime}$ in $\mathcal{X}_{[c]}^{\prime} A_{[k]}^{\prime}$ such that:

1. The state $\rho^{\prime}$ and POVM element $\Pi^{\prime}$ are classical on $\mathcal{X}^{\otimes[c]} \otimes \mathcal{L}^{[c] \cup[k]}$ and quantum on $A_{[k]}^{\prime \prime}$. More precisely, $\rho^{\prime}, \Pi^{\prime}$ can be expressed as

$$
\begin{gathered}
\left(\rho^{\prime}\right)^{X_{[c]}^{\prime} A_{[k]}^{\prime}}=|\mathcal{L}|^{-(c+k)} \sum_{\mathbf{x}, \mathbf{l}} p(\mathbf{x})|\mathbf{x}\rangle\left\langle\left.\mathbf{x}\right|^{X_{[c]}} \otimes \mid \mathbf{I}\right\rangle\left\langle\left.\mathbf{I}\right|^{\mathcal{L}_{[c] \cup[k]}}\right. \\
\otimes\left(\rho^{\prime}\right)_{\mathbf{x}, \mathbf{l}, \delta}^{A_{[k]}^{\prime \prime}}, \\
\left(\Pi^{\prime}\right)^{X_{[c]}^{\prime} A_{[k]}^{\prime}}=\sum_{\mathbf{x}, \mathbf{l}}|\mathbf{x}\rangle\left\langle\left.\mathbf{x}\right|^{X_{[c]}} \otimes \mid \mathbf{I}\right\rangle\left\langle\left.\mathbf{l}\right|^{\mathcal{L}_{[c] \cup[k]}} \otimes\left(\Pi^{\prime}\right)_{\mathbf{x}, \mathbf{l}, \delta}^{A_{[k]}^{\prime \prime}},\right.
\end{gathered}
$$

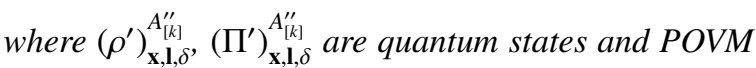
elements respectively for all computational basis vectors $\mathbf{x} \in \mathcal{X}^{\otimes[c]}, \mathbf{l} \in \mathcal{L}^{\otimes([c] \cup[k])}$;

2.

$$
\left\|\left(\rho^{\prime}\right)^{\mathcal{X}_{[c]}^{\prime} A_{[k]}^{\prime}}-\rho^{\mathcal{X}_{[c]} A_{[k]}} \otimes(|0\rangle\langle 0|)^{\left(\mathbb{C}^{2}\right)^{\otimes k}} \otimes \frac{\boldsymbol{1}^{\mathcal{L}^{8(c+k)}}}{|\mathcal{L}|^{c+k}}\right\|_{1} \leq 2^{\frac{c+k}{2}+1} \delta ;
$$

3.

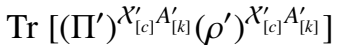

$$
\begin{aligned}
& \geq 1-\delta^{-2 k} 2^{2^{c k+4}(k+1)^{k}} \sum_{\left(S_{1}, \ldots, S_{l}\right) \vdash \vdash[c] \cup[k]} \epsilon_{\left(S_{1}, \ldots, S_{l}\right)}-2^{\frac{c+k}{2}+1} \delta ;
\end{aligned}
$$

4. Let $\left(S_{1}, \ldots, S_{l}\right) \vdash \vdash[c] \cup[k], l>0$. Define $T:=[k] \backslash$ $\left(S_{1} \cup \cdots \cup S_{l}\right)$. Let $\sigma_{\mathbf{x}}^{A_{T}}$ be a state in $A_{T}$. Let $S \subseteq[c] \cup$ $[k], S \cap[k] \neq\{\}$, Let $\mathbf{x}_{[c] \cap S}, \mathbf{l}_{S}$ be computational basis vectors in $\mathcal{X}^{\otimes([c] \cap S}, \mathcal{L}^{\otimes S}$. Let $p_{[c] \backslash S}(\cdot)$ be a probability distribution on $\mathcal{X}^{\otimes([c] \backslash S)}$. In the following definition, let $\mathbf{x}_{[c] \backslash S}^{\prime}, \mathbf{l}_{\bar{S}}^{\prime}$ range over all computational basis vectors of $\mathcal{X}^{\otimes([c] \backslash S)}, \mathcal{L}^{\otimes \bar{S}}$. Define a state in $A_{S \cap[k]}^{\prime \prime}$,

$$
\begin{aligned}
& \left(\rho^{\prime}\right)_{\mathbf{x}_{S \cap[c]}}^{A_{S \cap[S]}^{\prime \prime}, \mathbf{l}_{S}, \delta}
\end{aligned}
$$

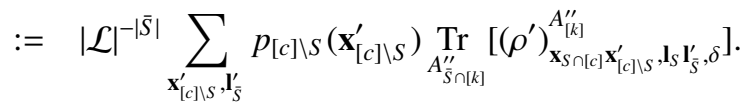

Analogously define

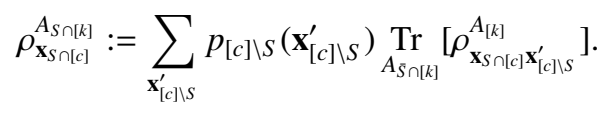

Define

$$
\begin{aligned}
\left(\rho^{\prime}\right)_{\mathbf{x}, \mathbf{l},\left(S_{1}, \ldots, S_{l}\right), \delta}^{A_{[k]}^{\prime \prime}} & \\
:=\quad\left(\rho_{\mathbf{x}_{S_{1} \cap[c]}^{\prime}, \mathbf{l}_{S_{1}}, \delta}^{\prime}\right. & )_{S_{1} \cap[k]}^{A^{\prime \prime}} \\
& \otimes \cdots \otimes
\end{aligned}
$$




$$
\begin{gathered}
\left(\rho_{\mathbf{x}_{S_{l} \cap[c]}, \mathbf{l}_{l}, \delta}^{\prime}\right)^{A_{S_{l} \cap[k]}^{\prime \prime}} \\
\otimes\left(\sigma_{\mathbf{x}}^{A_{T}} \otimes\left(|0\rangle\left\langle\left. 0\right|^{\mathbb{C}^{2}}\right)^{\otimes|T|}\right),\right. \\
\rho_{\mathbf{x},\left(S_{1}, \ldots, S_{l}\right)}^{A_{[k]}}:=\quad \rho_{\mathbf{x}_{S_{1} \cap[c]}}^{A_{S_{1} \cap[k]}} \otimes \cdots \otimes \rho_{\mathbf{x}_{S_{l} \cap[c]}}^{A_{S_{l} \cap[k]}} \otimes \sigma_{\mathbf{x}}^{A_{T}} .
\end{gathered}
$$

Let $q_{\left(S_{1}, \ldots, S_{l}\right)}(\cdot)$ be a probability distribution over vectors $\mathbf{x}$. Define

$$
\begin{aligned}
& \left(\rho^{\prime}\right)_{\left(S_{1}, \ldots, S_{l}\right)}^{X_{[c]}^{\prime} A_{[k]}^{\prime}} \\
& :=|\mathcal{L}|^{-(c+k)} \sum_{\mathbf{x}, \mathbf{l}} q_{\left(S_{1}, \ldots, S_{l}\right)}(\mathbf{x}) \\
& |\mathbf{x}\rangle\left\langle\left.\mathbf{x}\right|^{X_{[c]}} \otimes \mid \mathbf{I}\right\rangle\left\langle\left.\mathbf{l}\right|^{\mathcal{L}_{[c] \cup[k]}}\right. \\
& \otimes\left(\rho^{\prime}\right)_{\mathbf{x}, \mathbf{l},\left(S_{1, \ldots}\right.}^{A_{[k]}^{\prime \prime}} \\
& \rho_{\left(S_{1}, \ldots, S_{l}\right)}^{\mathcal{X}_{[c]} A_{[k]}}:=\sum_{\mathbf{x}} q_{\left(S_{1}, \ldots, S_{l}\right)}(\mathbf{x})|\mathbf{x}\rangle\left\langle\left.\mathbf{x}\right|^{X_{[c]}} \rho_{\mathbf{x},\left(S_{1}, \ldots, S_{l}\right)}^{A_{[k]}} .\right.
\end{aligned}
$$

Then,

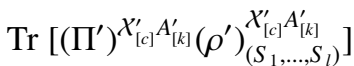

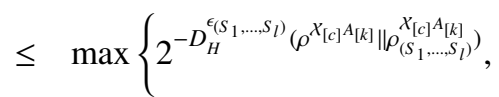

$$
\begin{aligned}
& \left.\frac{3(2|\mathcal{H}|)^{k}}{\sqrt{|\mathcal{L}|}}\right\} .
\end{aligned}
$$

Proof: The lemma is a direct consequence of Proposition 4. Consider first the augmented $k$-partite system $A_{1}^{\prime \prime} \cdots A_{k}^{\prime \prime}$. For computational basis vectors $\mathbf{x}, \mathbf{l}$ let $\left(\rho_{\mathbf{x}, \mathbf{l}, \delta}^{\prime}\right)^{A_{[k]}^{\prime \prime}}$ be the quantum state and $\left(\Pi^{\prime}\right)_{\mathbf{x}, \mathbf{l}, \delta}^{A_{k]}^{\prime \prime}}$ be the POVM element in $A_{[k]}^{\prime \prime}$ guaranteed by Existence Statements 4 and 4 of Proposition 4 . These quantities are used to define the state $\rho^{\prime}$ and POVM element $\Pi^{\prime}$ in $\mathcal{X}_{[c]}^{\prime} A_{[k]}^{\prime}$ as in Claim 1 of the lemma.

From Claim 3 of Proposition 4.

$$
\begin{aligned}
& \left\|\left(\rho^{\prime}\right)^{X_{[c]}^{\prime} A_{[k]}^{\prime}}-\rho^{X_{[c]} A_{[k]}} \otimes\left(|0\rangle\left\langle\left. 0\right|^{\mathbb{C}^{2}}\right)^{\otimes k} \otimes \frac{\mathbb{1}^{\mathcal{L}^{\otimes k}}}{|\mathcal{L}|^{k}} \|_{1}\right.\right. \\
& =\|\left(| \mathcal { L } | ^ { - ( c + k ) } \sum _ { \mathbf { x } , \mathbf { l } } p ( \mathbf { x } ) | \mathbf { x } \rangle \langle \mathbf { x } | ^ { X _ { [ c ] } } \otimes | \mathbf { I } \rangle \left\langle\left.\mathbf{l}\right|^{\mathcal{L}^{\otimes(k+c)}}\right.\right. \\
& \quad \otimes\left(\left(\rho^{\prime}\right)_{\mathbf{x}, \mathbf{l}, \delta}^{A_{[k]}^{\prime \prime}}-\rho^{A_{[k]}} \otimes\left(|0\rangle\left\langle\left. 0\right|^{\mathbb{C}^{2}}\right)^{\otimes k}\right)\right) \|_{1} \\
& \leq|\mathcal{L}|^{-(c+k)} \sum_{\mathbf{x}, \mathbf{l}} p(\mathbf{x})\left\|\left(\rho^{\prime}\right)_{\mathbf{x}, \mathbf{l}, \delta}^{A_{[k]}^{\prime \prime}}-\rho^{A_{[k]}} \otimes\left(|0\rangle\left\langle\left. 0\right|^{\mathbb{C}^{2}}\right)^{\otimes k} \|_{1}\right.\right. \\
& \leq \quad 2^{\frac{c+k}{2}+1} \delta,
\end{aligned}
$$

which proves Claim 2 of the lemma.

For each pseudosubpartition $\left(S_{1}, \ldots, S_{l}\right) \vdash \vdash[c] \cup[k]$, let $\Pi_{\left(S_{1}, \ldots, S_{l}\right)}^{X_{[c} A_{[k]}}$ be the optimising POVM element in the definition of $D_{H}^{\epsilon_{\left(S S_{1}, \ldots, S_{l}\right)}}\left(\rho^{\mathcal{X}_{[c]} A_{[k]}} \| \rho_{\left(S_{1}, \ldots, S_{l}\right)}^{\mathcal{X}_{[c]} A_{[k]}}\right)$. Without loss of generality, it is of the form

$$
\Pi_{\mathbf{x},\left(S_{1}, \ldots, S_{l}\right)}^{X_{[c} A_{[k]}}=\sum_{\mathbf{x}}|\mathbf{x}\rangle\left\langle\left.\mathbf{x}\right|^{X_{[c]}} \otimes \Pi_{\mathbf{x},\left(S_{1}, \ldots, S_{l}\right)}^{A_{[k]}}\right.
$$

where for each $\mathbf{x}, \Pi_{\mathbf{x},\left(S_{1}, \ldots, S_{l}\right)}$ is a POVM element in $A_{[k]}$. Define $\epsilon_{\mathbf{x},\left(S_{1}, \ldots, S_{l}\right)}:=1-\operatorname{Tr}\left[\Pi_{\mathbf{x},\left(S_{1}, \ldots, S_{l}\right)}^{A_{[k]}} \rho_{\mathbf{x}}^{\left.A_{[k]}\right]}\right]$. Then, $\Pi_{\mathbf{x},\left(S_{1}, \ldots, S_{l}\right)}^{A_{[k]}}$ is the optimising POVM element in the definition of

$$
D_{H}^{\epsilon_{\mathbf{x},\left(S_{1}, \ldots, S_{l}\right)}}\left(\rho_{\mathbf{x}}^{A_{[k]}} \| \rho_{\mathbf{x},\left(S_{1}, \ldots, S_{l}\right)}^{A_{[k]}}\right)
$$

This implies that

$$
\begin{aligned}
& \sum_{\mathbf{x}} p(\mathbf{x}) \epsilon_{\mathbf{x},\left(S_{1}, \ldots, S_{l}\right)} \\
& =1-\epsilon_{\left(S_{1}, \ldots, S_{l}\right)},
\end{aligned}
$$

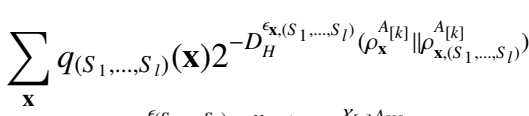

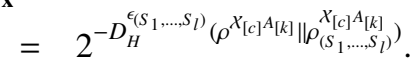

From Claims 4, 3 of Proposition 4

$$
\begin{aligned}
& \operatorname{Tr}\left[\left(\Pi^{\prime}\right)^{X_{[c]}^{\prime}} A_{[k]}^{\prime}\left(\rho^{\prime}\right)^{X_{[c]}^{\prime} A_{[k]}^{\prime}}\right]
\end{aligned}
$$

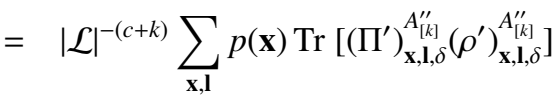

$$
\begin{aligned}
& \geq|\mathcal{L}|^{-(c+k)} \sum_{\mathbf{x}, \mathbf{l}} p(\mathbf{x})\left(\operatorname { T r } \left[\left(\Pi^{\prime}\right)_{\mathbf{x}, \mathbf{l}, \delta}^{A_{[k]}^{\prime \prime}}\left(\rho^{A_{[k]}} \otimes\left(|0\rangle\left\langle\left. 0\right|^{\mathbb{C}^{2}}\right)^{\otimes k}\right)\right]\right.\right. \\
& -\left\|\left(\rho^{\prime}\right)_{\mathbf{x}, \mathbf{l}, \delta}^{A_{[k]}^{\prime \prime}}-\rho^{A_{[k]}} \otimes\left(|0\rangle\left\langle\left. 0\right|^{\mathbb{C}^{2}}\right)^{\otimes k} \|_{1}\right)\right. \\
& \geq 1-\sum_{\mathbf{x}} p(\mathbf{x}) \delta^{-2 k} 2^{2^{c k+4}(k+1)^{k}} \sum_{\left(S_{1}, \ldots, S_{l}\right) \vdash \vdash[c] \cup[k]} \epsilon_{\mathbf{x},\left(S_{1}, \ldots, S_{l}\right)} \\
& -2^{\frac{k+c}{2}+1} \delta \\
& \geq 1-\delta^{-2 k} 2^{2^{c k+4}(k+1)^{k}} \sum_{\left(S_{1}, \ldots, S_{l}\right) \vdash[c] \cup[k]} \epsilon_{\left(S_{1}, \ldots, S_{l}\right)}-2^{\frac{k+c}{2}+1} \delta,
\end{aligned}
$$

which proves Claim 3 of the lemma.

Using Claims 5, 6, 1, 2 of Proposition 4 and Equation 9 , we get

$$
\begin{aligned}
& \left.\operatorname{Tr}\left[\left(\Pi^{\prime}\right)^{X_{[c]}^{\prime} A_{[k]}^{\prime}\left(\rho^{\prime}\right)}\right)_{\left(S_{1}, \ldots, S_{l}\right)}^{X_{c}^{\prime} A_{[k]}^{\prime}}\right]
\end{aligned}
$$

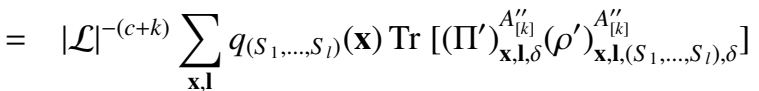

$$
\begin{aligned}
& =\alpha_{\left(S_{1}, \ldots, S_{l}\right), \delta} \\
& |\mathcal{L}|^{-(c+k)} \sum_{\mathbf{x}, \mathbf{l}} q_{\left(S_{1}, \ldots, S_{l}\right)}(\mathbf{x}) \\
& \operatorname{Tr}\left[( \Pi ^ { \prime } ) _ { \mathbf { x } , \mathbf { l } , \delta } ^ { A _ { [ k ] } ^ { \prime \prime } } \left(\mathcal{T}_{\left(S_{1}, \ldots S_{l}\right), \mathbf{l}, \delta}\left(\rho_{\mathbf{x},\left(S_{1}, \ldots, S_{l}\right)}^{A_{[k]}} \otimes\left(|0\rangle\left\langle\left. 0\right|^{\mathbb{C}^{2}}\right)^{\otimes k}\right)\right)^{\left.A_{[k]}^{\prime \prime}\right]}\right.\right. \\
& +\beta_{\left(S_{1}, \ldots, S_{l}\right), \delta} \\
& |\mathcal{L}|^{-(c+k)} \sum_{\mathbf{x}, \mathbf{l}} q_{\left(S_{1}, \ldots, S_{l}\right)}(\mathbf{x}) \operatorname{Tr}\left[\left(\Pi^{\prime}\right)_{\mathbf{x}, \mathbf{l}, \delta}^{A_{[k]}^{\prime \prime}} N_{\left(S_{1}, \ldots S_{l}\right), \mathbf{x}, \mathbf{l}, \delta}^{A_{[k]}^{\prime \prime}}\right] \\
& +\left(1-\alpha_{\left(S_{1}, \ldots, S_{l}\right), \delta}-\beta_{\left(S_{1}, \ldots, S_{l}\right), \delta}\right) \\
& |\mathcal{L}|^{-(c+k)} \sum_{\mathbf{x}, \mathbf{l}} q_{\left(S_{1}, \ldots, S_{l}\right)}(\mathbf{x}) \operatorname{Tr}\left[\left(\Pi^{\prime}\right)_{\mathbf{x}, \mathbf{l}, \delta}^{A_{[k]}^{\prime \prime}} M_{\left(S_{1}, \ldots S_{l}\right), \mathbf{x}, \mathbf{l}, \delta}^{A_{[k]}^{\prime \prime}}\right]
\end{aligned}
$$

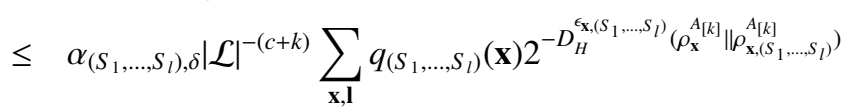

$$
\begin{aligned}
& +\beta_{\left(S_{1}, \ldots, S_{l}\right), \delta}|\mathcal{L}|^{-(c+k)} \\
& \sum_{\mathbf{x}, \mathbf{l}} q_{\left(S_{1}, \ldots, S_{l}\right)}(\mathbf{x})\left\|\left(\Pi^{\prime}\right)_{\mathbf{x}, \mathbf{l}, \delta}^{A_{[k]}^{\prime \prime}}\right\|_{1}\left\|N_{\left(S_{1}, \ldots S_{l}\right), \mathbf{x}, \mathbf{l}, \delta}^{A_{[k]}^{\prime \prime}}\right\|_{\infty}
\end{aligned}
$$




$$
\begin{aligned}
& +\left(1-\alpha_{\left(S_{1}, \ldots, S_{l}\right), \delta}-\beta_{\left(S_{1}, \ldots, S_{l}\right), \delta}\right) \\
& |\mathcal{L}|^{-(c+k)} \sum_{\mathbf{x}, \mathbf{l}} q_{\left(S_{1}, \ldots, S_{l}\right)}(\mathbf{x})\left\|\left(\Pi^{\prime}\right)_{\mathbf{x}, \mathbf{l}, \delta}^{A_{[k]}^{\prime \prime}}\right\|_{1}\left\|M_{\left(S_{1}, \ldots S_{l}\right), \mathbf{x}, \mathbf{l}, \delta}^{A_{[k]}^{\prime \prime}}\right\|_{\infty} \\
& \leq \quad \alpha_{\left(S_{1}, \ldots, S_{l}\right), \delta} 2^{-D_{H}^{\epsilon\left(S_{1}, \ldots, S_{l}\right)}\left(\rho_{\mathbf{x}}^{A_{[k]}} \| \rho_{\mathbf{x},\left(S_{1}, \ldots, S_{l}\right)}^{A_{[l]}}\right)} \\
& +\beta_{\left(S_{1}, \ldots, S_{l}\right), \delta} \frac{3(2|\mathcal{H}|)^{k}}{\sqrt{|\mathcal{L}|}} \\
& +\left(1-\alpha_{\left(S_{1}, \ldots, S_{l}\right), \delta}-\beta_{\left(S_{1}, \ldots, S_{l}\right), \delta}\right) \frac{(2|\mathcal{H}|)^{k}}{|\mathcal{L}|} \\
& \leq \max \left\{2^{-D_{H}^{\left.\epsilon_{(S}, \ldots, S_{l}\right)}\left(\rho^{A_{[k]}} \| \rho_{\left(S_{1}, \ldots, S_{l}\right)}^{A_{[k]}}\right)}, \frac{3(2|\mathcal{H}|)^{k}}{|\mathcal{L}|}\right\} .
\end{aligned}
$$

This proves Claim 4 of the lemma.

The proof of the one-shot classical quantum joint typicality lemma, intersection case is finally complete.

\section{Remark:}

Often in inner bound proofs in classical network information theory, one has an 'ideal' probability distribution $p\left(\mathbf{x}_{[c]} \mathbf{x}_{[k]}\right)$ on an alphabet $\mathcal{X}^{c+k}$ which has to be accepted with probability close to one, and for each subset $S \subseteq[k]$, a 'false' probability distribution

$$
q_{S}\left(\mathbf{x}_{[c]} \mathbf{x}_{S} \mathbf{x}_{[k] \backslash S}\right):=p\left(\mathbf{x}_{[c]}\right) p\left(\mathbf{x}_{S} \mid \mathbf{x}_{[c]}\right) p\left(\mathbf{x}_{[k] \backslash S} \mid \mathbf{x}_{[c]}\right),
$$

which should be accepted with as low probability as possible. The distribution $q_{S}(\cdot)$ is a product of the marginals on $\mathcal{X}_{S}$ and $\mathcal{X}_{[k] \backslash S}$ conditioned on $\mathcal{X}_{[c]}$, which gives rise to the name of conditional joint typicality lemma. For each $S \subseteq[k]$, one can take the optimal test for the above task, and then take the intersection of all the tests. The preceding lemma is a generalisation of the classical conditional joint typicality lemma with intersection, where the conditioning system continues to be classical but the remaining systems are allowed to be quantum. That is why we call it the one-shot classical quantum joint typicality lemma, intersection case.

When there is no classical system, i.e. $c=0$, Lemma 1 simplifies to the following statement.

Corollary 3 (Quant. joint typ. lem., intersec. case) Let $\mathcal{H}$, $\mathcal{L}$ be Hilbert spaces. Let $k$ be a positive integer. Let $A_{1} \cdots A_{k}$ be a k-partite system where each $A_{i}$ is isomorphic to $\mathcal{H}$. Let $\rho$ be a quantum state in $A_{[k]}$. Consider the augmented $k$-partite system $A_{1}^{\prime} \cdots A_{k}^{\prime}$ where each $A_{i}^{\prime} \cong A_{i}^{\prime \prime} \otimes \mathcal{L}$, and each $A_{i}^{\prime \prime}$ is defined as

$$
A_{i}^{\prime \prime}:=\left(\mathcal{H} \otimes \mathbb{C}^{2}\right) \oplus \bigoplus_{S: i \in S \subseteq[k]}\left(\mathcal{H} \otimes \mathbb{C}^{2}\right) \otimes \mathcal{L}^{\otimes|S|} .
$$

Below, $\mathbf{l}$ denotes a computational basis vector of $\mathcal{L}^{\otimes[k]}$. Let $\frac{\mathbb{1}^{\mathcal{L}^{\otimes k}}}{\mid \mathcal{L}^{k}}$ denote the completely mixed state on $k$ tensor copies of $\mathcal{L}$. View $\rho^{A_{[k]}} \otimes(|0\rangle\langle 0|)^{\left(\mathbb{C}^{2}\right)^{\otimes k}} \otimes \frac{\mathbb{1}^{\mathcal{L}^{\otimes k}}}{\mid \mathcal{L}^{k}}$ as a state in $A_{[k]}^{\prime}$ under the natural embedding viz. the embedding in the ith system is into the first summand of $A_{i}^{\prime \prime}$ defined above tensored with $\mathcal{L}$.

Let $0 \leq \epsilon, \delta \leq 1$. Then, there is a state $\rho^{\prime}$ and a POVM element $\Pi^{\prime}$ in $A_{[k]}^{\prime}$ such that:
1. The state $\rho^{\prime}$ and POVM element $\Pi^{\prime}$ are classical on $\mathcal{L}^{[k]}$ and quantum on $A_{[k]}^{\prime \prime}$. More precisely, $\rho^{\prime}, \Pi^{\prime}$ can be expressed as

$$
\begin{aligned}
\left(\rho^{\prime}\right)^{A_{[k]}^{\prime}} & =|\mathcal{L}|^{-k} \sum_{\mathbf{l}}|\mathbf{I}\rangle\left\langle\left.\mathbf{l}\right|^{\mathcal{L}_{[k]}} \otimes\left(\rho^{\prime}\right)_{\mathbf{l}, \delta}^{A_{[k]}^{\prime \prime}},\right. \\
\left(\Pi^{\prime}\right)^{A_{[k]}^{\prime}} & =\sum_{\mathbf{l}}|\mathbf{I}\rangle\left\langle\left.\mathbf{l}\right|^{\mathcal{L}_{[k]}} \otimes\left(\Pi^{\prime}\right)_{\mathbf{l}, \delta}^{A_{[k]}^{\prime \prime}}\right.
\end{aligned}
$$

where $\left(\rho^{\prime}\right)_{\mathbf{1}, \delta}^{A_{[k]}^{\prime \prime}},\left(\Pi^{\prime}\right)_{\mathbf{1}, \delta}^{A_{[k]}^{\prime \prime}}$ are quantum states and POVM elements respectively for all computational basis vectors $\mathbf{l} \in \mathcal{L}^{\otimes[k]}$;

2.

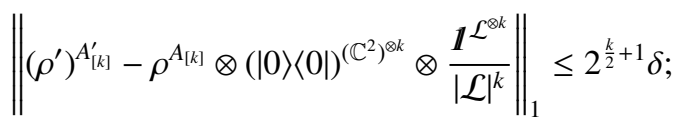

3.

$$
\operatorname{Tr}\left[\left(\Pi^{\prime}\right)^{A_{[k]}^{\prime}}\left(\rho^{\prime}\right)^{A_{[k]}^{\prime}}\right] \geq 1-\delta^{-2 k} 2^{17(k+1)^{k}} \epsilon-2^{\frac{k}{2}+1} \delta ;
$$

4. Let $\left(S_{1}, \ldots, S_{l}\right) \vdash[k], l>0$. Define $T:=[k] \backslash\left(S_{1} \cup\right.$ $\left.\cdots \cup S_{l}\right)$. Let $\sigma^{A_{T}}$ be a state in $A_{T}$. Let \{\}$\neq S \subseteq[k]$. Let $\mathbf{l}_{S}$ be computational basis vectors in $\mathcal{L}^{\otimes S}$. In the following definition, let $\mathbf{l}_{\bar{S}}^{\prime}$ range over all computational basis vectors of $\mathcal{L}^{\otimes \bar{S}}$. Define a state in $A_{S}^{\prime \prime}$,

$$
\left(\rho^{\prime}\right)_{\mathbf{l}_{S}, \delta}^{A_{S}^{\prime \prime}}:=|\mathcal{L}|^{-|\bar{S}|} \sum_{\mathbf{l}_{\bar{S}}^{\prime}} \operatorname{Tr}_{A_{\bar{S}}^{\prime \prime}}\left[\left(\rho^{\prime}\right)_{\mathbf{l}_{S} \mathbf{l}_{\bar{S}}^{\prime}, \delta}^{A_{\prime \prime}^{\prime \prime}}\right]
$$

Analogously define $\rho^{A_{S}}:=\operatorname{Tr}_{A_{\bar{S}}}\left[\rho^{A_{[k]}}\right]$. Define

$$
\begin{aligned}
\left(\rho^{\prime}\right)_{\mathbf{l},\left(S_{1}, \ldots, S_{l}\right), \delta}^{A_{[k]}^{\prime \prime}} & \\
:= & \left(\rho_{\mathbf{l}_{S_{1}}, \delta}^{\prime}\right)^{A_{S_{1}}^{\prime \prime}} \otimes \cdots \otimes\left(\rho_{\mathbf{l}_{S_{l}}, \delta}^{\prime}\right)^{A_{S_{l}}^{\prime \prime}} \\
& \otimes\left(\sigma^{A_{T}} \otimes\left(|0\rangle\left\langle\left. 0\right|^{\mathbb{C}^{2}}\right)^{\otimes|T|}\right),\right. \\
\rho_{\left(S_{1}, \ldots, S_{l}\right)}^{A_{[k]}}:= & \rho^{A_{S_{1}}} \otimes \cdots \otimes \rho^{A_{S_{l}}} \otimes \sigma^{A_{T}} .
\end{aligned}
$$

Define

$$
\left(\rho^{\prime}\right)_{\left(S_{1}, \ldots, S_{l}\right)}^{A_{[k]}^{\prime}}:=|\mathcal{L}|^{-k} \sum_{\mathbf{x}, \mathbf{l}}|\mathbf{I}\rangle\left\langle\left.\mathbf{l}\right|^{\mathcal{L}_{[k]}} \otimes\left(\rho^{\prime}\right)_{\mathbf{l},\left(S_{1}, \ldots, S_{l}\right), \delta}^{A_{[k}^{\prime \prime}} .\right.
$$

Then,

$$
\begin{aligned}
& \operatorname{Tr}\left[\left(\Pi^{\prime}\right)^{A_{[k]}^{\prime}}\left(\rho^{\prime}\right)_{\left(S_{1}, \ldots, S_{l}\right)}^{A_{[k]}^{\prime}}\right] \\
& \quad \leq \max \left\{2^{-D_{H}^{\epsilon}\left(\rho^{A}[k] \| \rho_{\left(S_{1}, \ldots, S_{l}\right)}^{A_{[k]}}\right)}, \frac{3(2 \mathcal{H})^{k}}{\sqrt{|\mathcal{L}|}}\right\} .
\end{aligned}
$$

\section{Remark:}

The statement of the joint typicality lemma given at the end of Section 1.4 easily follows from the more general statement in Corollary 3 using Proposition 1 . We first observe by 
Proposition 1 that for any state $\rho^{A_{[k]}}$ and any subset \{\}$\neq S \subset$ $[k]$,

$$
D_{H}^{\epsilon}\left(\rho^{A_{[k]}} \| \rho^{A_{S}} \otimes \rho^{A_{\bar{S}}}\right) \leq k \log |\mathcal{H}|+3 \log \frac{1}{1-\epsilon}+6 \log 3-4 .
$$

Choose $\mathcal{L}$ of dimension $\frac{3^{13}(2|\mathcal{H}|)^{4 k}}{2^{8}(1-\epsilon)^{6}}$ in Corollary 3 Choose the Hilbert space $\mathcal{K}$ to be of dimension

$$
2|\mathcal{L}|^{k+1}<\frac{2^{13(k+1)}(2|\mathcal{H}|)^{4 k(k+1)}}{(1-\epsilon)^{6(k+1)}} .
$$

This makes the dimension of $\mathcal{H} \otimes \mathcal{K}$ large enough to contain $A_{i}^{\prime}$. Choose $\delta:=\epsilon^{\frac{1}{4 k}}$. Define $\tau^{\mathcal{K}^{\otimes[k]}}:=(|0\rangle\langle 0|)^{\left(\mathbb{C}^{2}\right)^{\otimes[k]}} \otimes \frac{\mathbb{1}^{\mathcal{L}^{\otimes[k]}}}{\mid \mathcal{L}^{k}}$. The statement at the end of Section 1.4 now follows easily from Corollary 3

In Lemma 1, in the important special case of $k=1$, it is not necessary to augment the register $A_{1}$ with $\mathcal{L}$. This is because a pseudosubpartition of $[c] \cup[1]$ consists of only one subset which must contain the register $A_{1}$. This simplifies the proof of Lemma 1. In fact, we can prove the following joint typicality lemma in Corollary 4 which is quite useful in applications to network information theory. The lemmas allow the 'negative hypotheses' to be classical quantum states where the quantum register is either the average over some classical systems of the positive hypothesis or a fixed quantum state. The flexibility of allowing a fixed quantum state is with an eye to potential applications in Shannon theory like generalised quantum Slepian-Wolf [28], though the entropic quantity in Slepian-Wolf is of the covering type where our joint typicality lemmas do not seem to apply.

Corollary 4 (Useful joint typ. lem., intersec. case) Let $\mathcal{H}$, $\mathcal{L}$ be Hilbert spaces and $\mathcal{X}$ be a finite set. We will also use $\mathcal{X}$ to denote the Hilbert space with computational basis elements indexed by the set $\mathcal{X}$. Let $c$ be a non-negative integer. Let $A$ denote a quantum register with Hilbert space $\mathcal{H}$. For every $\mathbf{x} \in X^{c}$, let $\rho_{\mathbf{x}}$ be a quantum state in A. Consider the extended quantum system

$$
A^{\prime}:=\left(\mathcal{H} \otimes \mathbb{C}^{2}\right) \oplus \bigoplus_{S:\{\neq \neq \subseteq \subseteq[c]}\left(\mathcal{H} \otimes \mathbb{C}^{2}\right) \otimes \mathcal{L}^{\otimes|S|} .
$$

Also define the augmented classical system $\mathcal{X}^{\prime}:=\mathcal{X} \otimes \mathcal{L}$.

Below, $\mathbf{x}, \mathbf{l}$ denote computational basis vectors of $\mathcal{X}^{[c]}$, $\mathcal{L}^{\otimes[c]}$. Let $p(\cdot)$ be a probability distribution on the vectors $\mathbf{x}$. Define the classical quantum state

$$
\rho^{X_{[c]} A}:=\sum_{\mathbf{x}} p(\mathbf{x})|\mathbf{x}\rangle\left\langle\left.\mathbf{x}\right|^{X_{[c]}} \otimes \rho_{\mathbf{x}}^{A} .\right.
$$

Let $\frac{\mathbb{1}^{\mathcal{L}^{8 c}}}{|\mathcal{L}|^{c}}$ denote the completely mixed state on $c$ tensor copies of $\mathcal{L}$. View $\rho_{\mathbf{x}}^{A} \otimes(|0\rangle\langle 0|)^{\mathbb{C}^{2}}$ as a state in $A^{\prime}$ under the natural embedding viz. the embedding is into the first summand of $A^{\prime}$ defined above. Similarly, view $\rho^{X_{[c]} A} \otimes(|0\rangle\langle 0|)^{\left(\mathbb{C}^{2}\right)} \otimes \frac{\mathbb{1}^{\mathcal{L}^{\otimes c}}}{|\mathcal{L}|^{c}}$ as a state in $X_{[c]}^{\prime} A^{\prime}$ under the natural embedding.

Let $0 \leq \epsilon, \delta \leq 1$. Choose $\mathcal{L}$ to have dimension $|\mathcal{L}|=$ $\frac{3^{13}|\mathcal{H}|^{4}}{2^{4}(1-\epsilon)^{6}}$. Then, there is a state $\rho^{\prime}$ and a POVM element $\Pi^{\prime}$ in
$\mathcal{X}_{[c]}^{\prime} A^{\prime}$ such that:
1. The state $\rho^{\prime}$ and POVM element $\Pi^{\prime}$ are classical on $\mathcal{X}^{\otimes[c]} \otimes \mathcal{L}^{[c]}$ and quantum on $A^{\prime}$. More precisely, $\rho^{\prime}, \Pi^{\prime}$ can be expressed as

$$
\begin{aligned}
\left(\rho^{\prime}\right)^{X_{[c]}^{\prime} A^{\prime}} & =|\mathcal{L}|^{-c} \sum_{\mathbf{x}, \mathbf{l}} p(\mathbf{x})|\mathbf{x}\rangle\left\langle\left.\mathbf{x}\right|^{\mathcal{X}_{[c]}} \otimes \mid \mathbf{I}\right\rangle\left\langle\left.\mathbf{l}\right|^{\mathcal{L}_{[c]}} \otimes\left(\rho^{\prime}\right)_{\mathbf{x}, \mathbf{l}, \delta}^{A^{\prime}},\right. \\
\left(\Pi^{\prime}\right)^{X_{[c]}^{\prime} A^{\prime}} & =\sum_{\mathbf{x}, \mathbf{l}}|\mathbf{x}\rangle\left\langle\left.\mathbf{x}\right|^{\mathcal{X}_{[c]}} \otimes \mid \mathbf{I}\right\rangle\left\langle\left.\mathbf{l}\right|^{\mathcal{L}_{[c]}} \otimes\left(\Pi^{\prime}\right)_{\mathbf{x}, \mathbf{l}, \delta}^{A^{\prime}}\right.
\end{aligned}
$$

where $\left(\rho^{\prime}\right)_{\mathbf{x}, \mathbf{l}, \delta}^{A^{\prime}},\left(\Pi^{\prime}\right)_{\mathbf{x}, \mathbf{l}, \delta}^{A^{\prime}}$ are quantum states and POVM elements respectively for all computational basis vectors $\mathbf{x} \in \mathcal{X}^{\otimes[c]}, \mathbf{l} \in \mathcal{L}^{\otimes[c]}$;

2

$$
\left\|\left(\rho^{\prime}\right)^{X_{[c]}^{\prime} A^{\prime}}-\rho^{X_{[c]} A} \otimes(|0\rangle\langle 0|)^{\mathbb{C}^{2}} \otimes \frac{\mathbb{1}^{\mathcal{L}^{\otimes c}}}{|\mathcal{L}|^{c}}\right\|_{1} \leq 2^{\frac{c+1}{2}+1} \delta
$$

3.

$$
\operatorname{Tr}\left[\left(\Pi^{\prime}\right)^{X_{[c]}^{\prime} A^{\prime}}\left(\rho^{\prime}\right)^{X_{[c]}^{\prime} A^{\prime}}\right] \geq 1-\delta^{-2} 2^{2^{c+5}} 3^{c} \epsilon-2^{\frac{c+1}{2}+1} \delta ;
$$

4. Let $S \subseteq[c]$. Let $\mathbf{x}_{S}, \mathbf{l}_{S}$ be computational basis vectors in $\mathcal{X}^{\otimes S}, \mathcal{L}^{\otimes S}$. In the following definition, let $\mathbf{x}_{\bar{S}}^{\prime}, \mathbf{l}_{\bar{S}}^{\prime}$

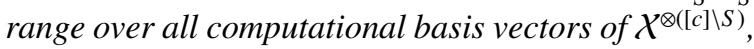
$\mathcal{L}^{\otimes([c] \backslash S)}$. Define a state in $A^{\prime}$,

$$
\left(\rho^{\prime}\right)_{\mathbf{x}_{S}, \mathbf{l}_{S}, \delta}^{A^{\prime}}:=|\mathcal{L}|^{-|\bar{S}|} \sum_{\mathbf{x}_{\bar{S}}^{\prime}, \mathbf{l}_{\bar{S}}^{\prime}} p\left(\mathbf{x}_{\bar{S}}^{\prime} \mid \mathbf{x}_{S}\right)\left(\rho^{\prime}\right)_{\mathbf{x}_{S} \mathbf{x}_{\bar{S}}^{\prime}, \mathbf{l}_{S} \mathbf{l}_{\bar{S}}^{\mathbf{S}_{S}, \delta}}^{A^{\prime}} .
$$

Analogously define

$$
\rho_{\mathbf{x}_{S}}^{A}:=\sum_{\mathbf{x}_{\bar{S}}^{\prime}} p\left(\mathbf{x}_{\bar{S}}^{\prime} \mid \mathbf{x}_{S}\right) \rho_{\mathbf{x}_{S} \mathbf{x}_{\bar{S}}^{\prime}}^{A} .
$$

Let $\left(S_{1}, S_{2}, S_{3}\right) \dashv[c]$. Let $\sigma^{A}$ be a fixed quantum state in A. If $S_{1}, S_{3}=\{\}$ and $S_{2}=[c]$, define

$$
\begin{aligned}
\left(\rho^{\prime}\right)_{(\{\},[c],\{\})}^{\mathcal{X}_{[c]}^{\prime} A^{\prime}} & :=|\mathcal{L}|^{-c} \sum_{\mathbf{x}} p(\mathbf{x})|\mathbf{x}\rangle\left\langle\left.\mathbf{x}\right|^{\mathcal{X}_{[c]}} \otimes \mid \mathbf{I}\right\rangle\left\langle\left.\mathbf{l}\right|^{\mathcal{L}_{[c]}}\right. \\
& \otimes\left(\sigma^{A} \otimes|0\rangle\left\langle\left. 0\right|^{\mathbb{C}^{2}}\right),\right. \\
\rho_{(\{\},[c],\{\})}^{\mathcal{X}_{[c]} A} & :=\sum_{\mathbf{x}} p(\mathbf{x})|\mathbf{x}\rangle\left\langle\left.\mathbf{x}\right|^{X_{[c]}} \otimes \sigma^{A} .\right.
\end{aligned}
$$

Otherwise, define

$$
\begin{aligned}
&\left.\left(\rho^{\prime}\right)\right)_{\left(S_{1}, S_{2}, S_{3}\right)}^{X_{[c}^{\prime} A^{\prime}} \\
&:=|\mathcal{L}|^{-c} \sum_{\mathbf{x}_{S_{1}}} p\left(\mathbf{x}_{S_{1}}\right)\left|\mathbf{x}_{S_{1}}\right\rangle\left\langle\left.\mathbf{x}_{S_{1}}\right|^{X_{S_{1}}}\right. \\
& \otimes\left|\mathbf{l}_{S_{1}}\right\rangle\left\langle\left.\mathbf{l}_{S_{1}}\right|^{\mathcal{L}_{S_{1}}}\right.\left(\sum _ { \mathbf { x } _ { S _ { 2 } } } p ( \mathbf { x } _ { S _ { 2 } } | \mathbf { x } _ { S _ { 1 } } ) | \mathbf { x } _ { S _ { 2 } } \rangle \left\langle\left.\mathbf{x}_{S_{2}}\right|^{X_{S_{2}}}\right.\right. \\
& \otimes\left|\mathbf{l}_{S_{2}}\right\rangle\left\langle\left.\mathbf{l}_{S_{2}}\right|^{\left.\mathcal{L}_{S_{2}}\right)}\right.
\end{aligned}
$$




$$
\begin{array}{r}
\otimes\left(\sum _ { \mathbf { x } _ { S _ { 3 } } } p ( \mathbf { x } _ { S _ { 3 } } | \mathbf { x } _ { S _ { 1 } } ) | \mathbf { x } _ { S _ { 3 } } \rangle \left\langle\left.\mathbf{x}_{S_{3}}\right|^{X_{S_{3}}}\right.\right. \\
\otimes\left|\mathbf{l}_{S_{3}}\right\rangle\left\langle\mathbf{l}_{S_{3}} \mathcal{L}_{S_{3}}\right. \\
\otimes\left(\rho^{\prime}\right)_{\left.\mathbf{x}_{S_{1} \cup S_{3}}^{A^{\prime}}, \mathbf{l}_{S_{1} \cup S_{3}, \delta}\right),}^{\mathcal{X}_{[c]} A} \rho_{\left(S_{1}, S_{2}, S_{3}\right)}=\sum_{\mathbf{x}_{S_{1}}} p\left(\mathbf{x}_{S_{1}}\right)\left|\mathbf{x}_{S_{1}}\right\rangle\left\langle\left.\mathbf{x}_{S_{1}}\right|^{X_{S_{1}}}\right. \\
\otimes\left(\sum_{\mathbf{x}_{S_{2}}} p\left(\mathbf{x}_{S_{2}} \mid \mathbf{x}_{S_{1}}\right)\left|\mathbf{x}_{S_{2}}\right\rangle\left\langle\left.\mathbf{x}_{S_{2}}\right|^{X_{S_{2}}}\right)\right. \\
\otimes\left(\sum _ { \mathbf { x } _ { S _ { 3 } } } p ( \mathbf { x } _ { S _ { 3 } } | \mathbf { x } _ { S _ { 1 } } ) | \mathbf { x } _ { S _ { 3 } } \rangle \left\langle\left.\mathbf{x}_{S_{3}}\right|^{X_{S_{3}}}\right.\right. \\
\left.\otimes \rho_{\mathbf{x}_{S_{1} \cup S_{3}}}^{A}\right) .
\end{array}
$$

Then,

$$
\begin{gathered}
\operatorname{Tr}\left[\left(\Pi^{\prime}\right)^{X_{[c]}^{\prime} A^{\prime}}\left(\rho^{\prime}\right)_{\left(S_{1}, S_{2}, S_{3}\right)}^{X_{[c}^{\prime} A^{\prime}}\right] \leq 2^{-I_{H}^{\epsilon}\left(X_{S_{2}}: A X_{S_{3}} \mid X_{S_{1}}\right)_{\rho}}, \\
\text { where } I_{H}^{\epsilon}\left(X_{S_{2}}: A X_{S_{3}} \mid X_{S_{1}}\right)_{\rho}:=D_{H}^{\epsilon}\left(\rho^{X_{[c]} A} \| \rho_{\left(S_{1}, S_{2}, S_{3}\right)}^{X_{[c]} A}\right) .
\end{gathered}
$$

We now state our most general one-shot classical quantum joint typicality lemma.

Theorem 1 (cq joint typ. lem., gen. case) Let $\mathcal{H}, \mathcal{L}$ be Hilbert spaces and $\mathcal{X}$ be a finite set. We will also use $\mathcal{X}$ to denote the Hilbert space with computational basis elements indexed by the set $X$. Let $c$ be a non-negative and $k$ a positive integer. Let $A_{1} \cdots A_{k}$ be a k-partite system where each $A_{i}$ is isomorphic to $\mathcal{H}$. Let $t$ be a positive integer. Let $\mathbf{x}^{t}$ denote a $t$-tuple of elements of $\mathcal{X}^{c}$; we shall denote its ith element by $\mathbf{x}^{t}(i)$. Consider the extended $k$-partite system $\hat{A}_{1} \cdots \hat{A}_{k}$ where each $\hat{A_{i}} \cong A_{i}^{\prime} \otimes \mathbb{C}^{2} \otimes \mathbb{C}^{t+1}, A_{i}^{\prime} \cong A_{i}^{\prime \prime} \otimes \mathcal{L}$, and each $A_{i}^{\prime \prime}$ is defined as

$$
A_{i}^{\prime \prime}:=\left(\mathcal{H} \otimes \mathbb{C}^{2}\right) \oplus \bigoplus_{S: i \in S \subseteq[c] \cup[k]}\left(\mathcal{H} \otimes \mathbb{C}^{2}\right) \otimes \mathcal{L}^{\otimes|S|} .
$$

Also define $\hat{\mathcal{X}}:=\mathcal{X} \otimes \mathcal{L}$.

Below, $\mathbf{x}$ denotes computational basis vectors of $\mathcal{X}^{[c]}$, and $\mathbf{I}$ denotes computational basis vectors of $\mathcal{L}^{\otimes s}$ where $s$ will be clear from the context. Let $p(\cdot)$ denote a probability distribution on the vectors $\mathbf{x}$. Let $p(1 ; \cdot), \ldots, p(t ; \cdot)$ denote probability distributions on $\mathbf{x}^{t}$ such that the marginal of $p\left(i ; \mathbf{x}^{t}\right)$ on the ith element is $p\left(\mathbf{x}^{t}(i)\right)$. For $i \in[t]$, define the classical quantum states

$$
\rho^{\left(X_{[c]}\right)^{t} A_{[k]}}(i):=\sum_{\mathbf{x}^{t}} p\left(i ; \mathbf{x}^{t}\right)\left|\mathbf{x}^{t}\right\rangle\left\langle\left.\mathbf{x}^{t}\right|^{\left(X_{[c]}\right)^{t}} \otimes \rho_{\mathbf{x}^{t}(i)}^{A_{[k]}} .\right.
$$

Let $\frac{\mathbb{1}^{\Upsilon^{\otimes k}}}{\mid \mathcal{L}^{k}}$ denote the completely mixed state on $(c+k)$ tensor copies of $\mathcal{L}$. View $\rho_{\mathbf{x}}^{A_{[k]}}(i) \otimes(|0\rangle\langle 0|)^{\left(\mathbb{C}^{2}\right)^{\otimes k}} \otimes \frac{\mathbb{1}^{\mathcal{L}^{\otimes k}}}{|\mathcal{L}|^{k}} \otimes(|0\rangle\langle 0|)^{\left(\mathbb{C}^{2}\right)^{\otimes k}} \otimes$ $(|0\rangle\langle 0|)^{\left(\mathbb{C}^{t+1}\right)^{\otimes k}}$ as a state in $\hat{A}_{[k]}$ under the natural embedding viz. the embedding in the jth system is into the first summand of $A_{j}^{\prime \prime}$ defined above tensored with $\mathcal{L} \otimes \mathbb{C}^{2} \otimes \mathbb{C}^{t+1}$. Similarly, view $(\rho(i))^{\left(\mathcal{X}_{[c]}\right)^{t} A_{[k]}} \otimes(|0\rangle\langle 0|)^{\left(\mathbb{C}^{2}\right)^{\otimes k}} \otimes \frac{\boldsymbol{I}^{\mathcal{L}^{\otimes(c t+k)}}}{\mid \mathcal{L}^{c t+k}} \otimes(|0\rangle\langle 0|)^{\left(\mathbb{C}^{2}\right)^{\otimes k}} \otimes$ $(|0\rangle\langle 0|)^{\left(\mathbb{C}^{t+1}\right)^{\otimes k}}$ as a state in $\left(\hat{\mathcal{X}}_{[c]}\right)^{t} \hat{A}_{[k]}$ under the natural embedding.

Let $0 \leq \alpha, \epsilon, \delta \leq 1$. For each pseudosubpartition

$\left(S_{1}, \ldots, S_{l}\right) \vdash \vdash[c] \cup[k]$ and each $i \in[t]$, let $0 \leq \epsilon_{i,\left(S_{1}, \ldots, S_{l}\right)} \leq 1$. Then, there are states $\rho^{\prime}(1), \ldots, \rho^{\prime}(t)$ and a POVM element $\hat{\Pi}$ in $\left(\hat{\mathcal{X}}_{[c]}\right)^{t} \hat{A}_{[k]}$ such that:

1. The states $\rho^{\prime}(1), \ldots, \rho^{\prime}(t)$ and POVM element $\hat{\Pi}$ are classical on $\mathcal{X}^{\otimes[c t]} \otimes \mathcal{L}^{[c t] \cup[k]}$ and quantum on $A_{[k]}^{\prime \prime} \otimes$ $\left(\mathbb{C}^{2}\right)^{\otimes[k]} \otimes\left(\mathbb{C}^{t+1}\right)^{\otimes[k]}$. More precisely, $\rho^{\prime}(i), i \in[t], \hat{\Pi}$ can be expressed as

$$
\begin{aligned}
& \left(\rho^{\prime}(i)\right)^{\left(\hat{X}_{[c]}\right)^{t} \hat{A}_{[k]}} \\
& =|\mathcal{L}|^{-(c t+k)} \sum_{\mathbf{x}^{t}, \mathbf{l}} p\left(i ; \mathbf{x}^{t}\right)\left|\mathbf{x}^{t}\right\rangle\left\langle\left.\mathbf{x}^{t}\right|^{\left(X_{[c]}\right)^{t}} \otimes \mid \mathbf{I}\right\rangle\left\langle\left.\mathbf{I}\right|^{\mathcal{L}_{[t c] \cup[k]}}\right. \\
& \otimes\left(\rho^{\prime}\right)_{\mathbf{x}_{[k]}^{t}(i), \mathbf{l}_{[c]]}^{\prime \prime}(i) \mathbf{I}_{[k]}, \delta}^{A^{\prime \prime}} \\
& \otimes(|0\rangle\langle 0|)^{\left(\mathbb{C}^{2}\right)^{\otimes k}} \otimes(|0\rangle\langle 0|)^{\left(\mathbb{C}^{t+1}\right)^{\otimes k}}, \\
& \left(\Pi^{\prime}\right)^{\left(\hat{X}_{[c]}\right)^{t} \hat{A}_{[k]}} \\
& =\sum_{\mathbf{x}, \mathbf{l}}\left|\mathbf{x}^{t}\right\rangle\left\langle\left.\mathbf{x}^{t}\right|^{\left(X_{[c]}\right)^{t}} \otimes \mid \mathbf{I}\right\rangle\left\langle\left.\mathbf{I}\right|^{\mathcal{L}_{[c]] \cup[k]}}\right. \\
& \otimes(\hat{\Pi})_{\mathbf{x}^{t}, \mathbf{l}, \delta}^{A^{\prime \prime} \otimes\left(\mathbb{C}^{2}\right)^{\otimes[k]} \otimes\left(\mathbb{C}^{t+1}\right)^{\otimes[k]},},
\end{aligned}
$$

where $\left(\rho^{\prime}\right)_{\mathbf{x}, \mathbf{l}, \delta}^{A_{[k]}^{\prime \prime}}$ are quantum states for all computational basis vectors $\mathbf{x} \in \mathcal{X}^{\otimes[c]}, \mathbf{l} \in \mathcal{L}^{\otimes([c] \cup[k])}$ and

$$
\left(\Pi^{\prime}\right)_{\mathbf{x}^{t}, \mathbf{l}, \delta}^{A_{[k}^{\prime \prime} \otimes\left(\mathbb{C}^{2}\right)^{\otimes[k]} \otimes\left(\mathbb{C}^{t+1}\right)^{\otimes[k]}}
$$

are POVM elements for all computational basis vectors $\mathbf{x}^{t} \in \mathcal{X}^{\otimes[c t]}, \mathbf{l} \in \mathcal{L}^{\otimes([c t] \cup[k])}$;

2. For all $i \in[t]$,

$$
\begin{aligned}
& \|\left(\rho^{\prime}(i)\right)^{\left(\hat{X}_{[c]}\right)^{t} \hat{A}_{[k]}} \\
& -(\rho(i))^{\left(X_{[c]}\right)^{t} A_{[k]}} \otimes(|0\rangle\langle 0|)^{\left(\mathbb{C}^{2}\right)^{\otimes k}} \otimes \frac{\mathbb{1}^{\mathcal{L}^{\otimes(c t+k)}}}{|\mathcal{L}|^{t c+k}} \\
& \otimes(|0\rangle\langle 0|)^{\left(\mathbb{C}^{2}\right)^{\otimes k}} \otimes(|0\rangle\langle 0|)^{\left(\mathbb{C}^{t+1}\right)^{\otimes k}} \|_{1} \\
& \leq \quad 2^{\frac{c+k}{2}+1} \delta
\end{aligned}
$$

3. For all $i \in[t]$,

$$
\begin{aligned}
& \operatorname{Tr}\left[(\hat{\Pi})^{\left(\hat{X}_{[c]}\right)^{t} \hat{A}_{[k]}}\left(\rho^{\prime}(i)\right)^{\left(\hat{X}_{[c]}\right)^{t} \hat{A}_{[k]}}\right] \\
& \geq 1-\delta^{-2 k} 2^{2^{c k+4}(k+1)^{k}} \sum_{\left(S_{1}, \ldots, S_{l}\right) \vdash \vdash[c] \cup[k]} \epsilon_{i,\left(S_{1}, \ldots, S_{l}\right)} \\
& -2^{\frac{c+k}{2}+1} \delta-\alpha
\end{aligned}
$$

4. Let $\left(S_{1}, \ldots, S_{l}\right) \vdash \vdash[c] \cup[k], l>0$. Define $T:=[k] \backslash$ $\left(S_{1} \cup \cdots \cup S_{l}\right)$. Let $\sigma_{\mathbf{x}}^{A_{T}}$ be a state in $A_{T}$. Let $S \subseteq[c] \cup$ $[k], S \cap[k] \neq\{\}$, Let $\mathbf{x}_{[c] \cap S}, \mathbf{l}_{S}$ be computational basis vectors in $\mathcal{X}^{\otimes([c] \cap S}, \mathcal{L}^{\otimes S}$. Let $p_{[c] \backslash S}(\cdot)$ be a probability distribution on $\mathcal{X}^{\otimes([c] \backslash S)}$. In the following definition, let 
$\mathbf{x}_{[c] \backslash S}^{\prime}, \mathbf{l}_{\bar{S}}^{\prime}$ range over all computational basis vectors of $\mathcal{X}^{\otimes([c] \backslash S)}, \mathcal{L}^{\otimes \bar{S}}$. Define a state in $A_{S \cap[k]}^{\prime \prime}$,

$\left(\rho^{\prime}\right)_{\mathbf{x}_{S \cap[c]} \mathbf{l}_{S, \delta}^{\prime \prime}, \delta}^{\mathbf{l}_{S}}$

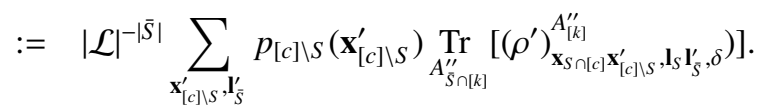

Analogously define

$$
\rho_{\mathbf{x}_{S \cap[c]}}^{A_{S \cap[k]}}:=\sum_{\mathbf{x}_{[c] \backslash S}^{\prime}} p_{[c] \backslash S}\left(\mathbf{x}_{[c] \backslash S}^{\prime}\right) \underset{A_{\bar{S} \cap[k]}}{\operatorname{Tr}}\left[\rho_{\mathbf{x}_{S \cap[c]} \mathbf{x}_{[c] \backslash S}^{\prime}}^{A_{[k]}}\right] .
$$

Define

$$
\begin{aligned}
\left(\rho^{\prime}\right)_{\mathbf{x}, \mathbf{l},\left(S_{1}, \ldots, S_{l}\right), \delta}^{A_{[k]}^{\prime \prime}} & \\
:= & \left(\rho_{\mathbf{x}_{S_{1} \cap[c]}^{\prime}, \mathbf{l}_{S_{1}, \delta}}^{\prime}\right)^{A_{S_{1} \cap[k]}^{\prime \prime}} \\
& \otimes \cdots \otimes \\
& \left(\rho_{\mathbf{x}_{\left.S_{l} \cap[c]\right)}^{\prime}, \mathbf{l}_{S_{l}, \delta}}^{\prime}\right)^{A_{S_{l} \cap[k]}^{\prime \prime}} \\
& \otimes\left(\sigma_{\mathbf{x}}^{A_{T}} \otimes\left(|0\rangle\left\langle\left. 0\right|^{\mathbb{C}^{2}}\right)^{\otimes[T]}\right),\right. \\
\rho_{\mathbf{x},\left(S_{1}, \ldots, S_{l}\right)}^{A_{[k]}}:= & \rho_{\mathbf{x}_{S_{1} \cap[c]}}^{A_{S_{1} \cap[k]}} \otimes \cdots \otimes \rho_{\mathbf{x}_{S_{l} \cap[c]}}^{A_{S_{l} \cap[k]}} \otimes \sigma_{\mathbf{x}}^{A_{T}} .
\end{aligned}
$$

For $i \in[t]$, let $q_{i ;\left(S_{1}, \ldots, S_{l}\right)}(\cdot)$ be a probability distribution over $\mathbf{x}^{t}$. Define

$$
\begin{aligned}
& \left(\rho^{\prime}\right)_{i ;\left(S_{1}, \ldots, S_{l}\right)}^{\left(\hat{X}_{[c]}\right)^{t} \hat{A}_{[k]}} \\
& :=|\mathcal{L}|^{-(c t+k)} \sum_{\mathbf{x}^{t}, \mathbf{l}} q_{i ;\left(S_{1}, \ldots, S_{l}\right)}\left(\mathbf{x}^{t}\right)\left|\mathbf{x}^{t}\right\rangle\left\langle\left.\mathbf{x}^{t}\right|^{\left(X_{[c]}\right)^{t}}\right. \\
& \otimes|\mathbf{I}\rangle\left\langle\left.\mathbf{l}\right|^{\mathcal{L}_{[c t] \cup[k]}}\right. \\
& \otimes\left(\rho^{\prime}\right)_{\mathbf{x}^{t}(i), \mathbf{l}_{[c t]}^{\prime \prime}(i),\left(S_{1}, \ldots, S_{l}\right), \delta}^{A^{\prime \prime}} \\
& \otimes(|0\rangle\langle 0|)^{\left(\mathbb{C}^{2}\right)^{\otimes k}} \otimes(|0\rangle\langle 0|)^{\left(\mathbb{C}^{t+1}\right)^{\otimes k}}, \\
& \rho_{i ;\left(S_{1}, \ldots, S_{l}\right)}^{\left(\mathcal{X}_{[c]}\right)^{t} A_{[k]}} \\
& :=\sum_{\mathbf{x}} q_{i ;\left(S_{1}, \ldots, S_{l}\right)}\left(\mathbf{x}^{t}\right)\left|\mathbf{x}^{t}\right\rangle\left\langle\left.\mathbf{x}^{t}\right|^{\left(X_{[c]}\right)^{t}} \otimes \rho_{\mathbf{x}^{t}(i),\left(S_{1}, \ldots, S_{l}\right)^{\prime}}^{A_{[k]}} .\right.
\end{aligned}
$$

Then,

$$
\begin{aligned}
& \operatorname{Tr}\left[(\hat{\Pi})^{\left(\hat{X}_{[c]}\right)^{t} \hat{A}_{[k]}}\left(\rho^{\prime}\right)_{i ;\left(S_{1}, \ldots, S_{l}\right)}^{\left(\hat{X}_{[c]}\right)^{t} \hat{A}_{[k]}}\right] \\
& \leq \frac{1-\alpha}{\alpha} \sum_{j=1}^{t}
\end{aligned}
$$

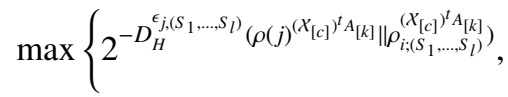

$$
\begin{aligned}
& \left.\frac{3(2|\mathcal{H}|)^{k}}{\sqrt{|\mathcal{L}|}}\right\} .
\end{aligned}
$$

Proof: For each $\mathbf{x} \in \mathcal{X}_{[c]}, \mathbf{l} \in \mathcal{L}_{[c] \cup[k]}$, construct the state $\left(\rho^{\prime}\right)_{\mathbf{x}, \mathbf{l}, \delta}^{A_{[k]}^{\prime \prime}}$ and POVM element $\left(\Pi^{\prime}\right)_{\mathbf{x}, \mathbf{l}, \delta}^{A_{[k]}^{\prime \prime}}$ as in Claim 1 of Lemma 1 For each $\mathbf{x}^{t} \in\left(\mathcal{X}_{[c]}\right)^{t}, \mathbf{l} \in \mathcal{L}_{[c t] \cup[k]}, i \in[t]$ define the POVM element

$$
\left(\Pi^{\prime}(i)\right)_{\mathbf{x}^{t}, \mathbf{1}, \delta}^{A_{1}^{\prime \prime}}:=\left(\Pi^{\prime}\right)_{\mathbf{x}^{t}(i), \mathbf{l}_{[c t]}^{\prime \prime}(i), \delta}^{A^{\prime \prime}}
$$

By Fact 2. there exists an orthogonal projector $(\hat{\Pi}(i))_{\mathbf{x}^{t}, 1, \delta}^{A_{1 \prime}^{\prime \prime} \otimes\left(\mathbb{C}^{2}\right)^{\otimes[k]}}$ such that

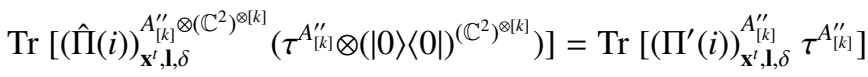

for all states $\tau^{A_{[k]}^{\prime \prime}}$. The projector $(\hat{\Pi})_{\mathbf{x}^{t}, \mathbf{l}, \delta}^{A_{k, \delta}^{\prime \prime} \otimes\left(\mathbb{C}^{2}\right)^{\otimes[k]} \otimes\left(\mathbb{C}^{t+1}\right)^{\otimes[k]}}$ is now constructed from the projectors $(\hat{\Pi}(i))_{\mathbf{x}^{t}, 1, \delta}^{A_{[k}^{\prime \prime} \otimes\left(\mathbb{C}^{2}\right)^{\otimes[k]}}, i \in[t]$ using Proposition 2. This settles Claim 1 of the theorem.

Claims 2 and 3 of the theorem follow from the corresponding Claims 2 and 3 of Lemma 1. Claim 4 of the theorem follows from Claim 4 of Lemma 1 and Proposition 2. combined with the observation that averaging over $\mathbf{x} \in \mathcal{X}_{[c]}$, $\mathbf{l} \in \mathcal{L}_{[c] \cup[k]}$ does not affect the $\left(S_{1}, \ldots, S_{l}\right)$ 'structure' of the state $\left(\rho^{\prime}\right)_{\mathbf{x}, \mathbf{l},\left(S_{1}, \ldots, S_{l}\right), \delta}^{A_{[\prime \prime}^{\prime \prime}}$

\section{Remark:}

The above theorem is a generalisation of Fact 1 to the classical quantum setting involving 'union of intersection of POVM elements'. However, it has the shortcoming that it can only handle classical quantum ' $q(\cdot)$ ' states corresponding to pseudosubpartitions of $[c] \cup[k]$, unlike Fact 1 which can handle any classical ' $q(\cdot)$ ' state. Overcoming this shortcoming remains an important open problem.

As remarked before Corollary 4 , in the important special case of $k=1$, it is not necessary to augment the register $A_{1}$ with $\mathcal{L}$. Thus, we can prove the following joint typicality lemma which is very useful in applications to network information theory.

Corollary 5 (Useful joint typicality lemma, general case) Let $\mathcal{H}, \mathcal{L}$ be Hilbert spaces and $\mathcal{X}$ be a finite set. We will also use $\mathcal{X}$ to denote the Hilbert space with computational basis elements indexed by the set $\mathcal{X}$. Let $c$ be a non-negative integer. Let $A$ denote a quantum register with Hilbert space $\mathcal{H}$. For every $\mathbf{x} \in \mathcal{X}^{c}$, let $\rho_{\mathbf{x}}$ be a quantum state in $A$. Let $t$ be a positive integer. Let $\mathbf{x}^{t}$ denote a $t$-tuple of elements of $X^{c} ;$ we shall denote its ith element by $\mathbf{x}^{t}(i)$. Consider the extended quantum system $\hat{A}$ where $\hat{A} \cong A^{\prime} \otimes \mathbb{C}^{2} \otimes \mathbb{C}^{t+1}$, and $A^{\prime}$ is defined as

$$
A^{\prime}:=\left(\mathcal{H} \otimes \mathbb{C}^{2}\right) \oplus \bigoplus_{S: i \in S \subseteq[c] \cup[k]}\left(\mathcal{H} \otimes \mathbb{C}^{2}\right) \otimes \mathcal{L}^{\otimes|S|} .
$$

Also define the augmented classical system $\hat{X}:=\mathcal{X} \otimes \mathcal{L}$.

Below, $\mathbf{x}, \mathbf{1}$ denote computational basis vectors of $\mathcal{X}^{[c]}$, $\mathcal{L}^{\otimes[c]}$. Let $p(\cdot)$ denote a probability distribution on the vectors x. Let $p(1 ; \cdot), \ldots, p(t ; \cdot)$ denote probability distributions on $\mathbf{x}^{t}$ such that the marginal of $p\left(i ; \mathbf{x}^{t}\right)$ on the ith element is $p\left(\mathbf{x}^{t}(i)\right)$. For $i \in[t]$, define the classical quantum states

$$
\rho^{\left(X_{[c]}\right)^{t} A}(i):=\sum_{\mathbf{x}^{t}} p\left(i ; \mathbf{x}^{t}\right)\left|\mathbf{x}^{t}\right\rangle\left\langle\left.\mathbf{x}^{t}\right|^{\left(X_{[c]}\right)^{t}} \otimes \rho_{\mathbf{x}^{t}(i)}^{A}\right.
$$

Let $\frac{\mathbb{1}^{\mathcal{L}^{\otimes c}}}{\mid \mathcal{L}^{c}}$ denote the completely mixed state on $\mathrm{c}$ tensor copies of $\mathcal{L}$. View $\rho_{\mathbf{x}}^{A} \otimes(|0\rangle\langle 0|)^{\mathbb{C}^{2}} \otimes(|0\rangle\langle 0|)^{\mathbb{C}^{2}} \otimes(|0\rangle\langle 0|)^{\mathbb{C}^{t+1}}$ as a state in $\hat{A}$ under the natural embedding viz. the embedding is into the first summand of $A^{\prime}$ defined above tensored with $\mathbb{C}^{2} \otimes \mathbb{C}^{t+1}$. 
Similarly, view $\rho^{\left(\mathcal{X}_{[c]}\right)^{t} A}(i) \otimes(|0\rangle\langle 0|)^{\mathbb{C}^{2}} \otimes \frac{\mathbb{1}^{\mathcal{L}^{\otimes c t}}}{\mid \mathcal{L}^{\mid c t}} \otimes(|0\rangle\langle 0|)^{\mathbb{C}^{2}} \otimes$ $(|0\rangle\langle 0|)^{\mathbb{C}^{t+1}}$ as a state in $\left(\hat{X}_{[c]}\right)^{\otimes t} \hat{A}$ under the natural embedding.

Let $0 \leq \alpha, \epsilon, \delta \leq 1$. Choose $\mathcal{L}$ to have dimension $|\mathcal{L}|=$ $\frac{3^{13}|\mathcal{H}|^{4}}{2^{4}(1-\epsilon)^{6}}$. Then, there are states $\rho^{\prime}(1), \ldots, \rho^{\prime}(t)$ and a POVM element $\hat{\Pi}$ in $\left(\hat{\mathcal{X}}_{[c]}\right)^{\otimes t} \hat{A}$ such that:

1. The states $\rho^{\prime}(1), \ldots, \rho^{\prime}(t)$ and POVM element $\hat{\Pi}$ are classical on $\mathcal{X}^{\otimes[c t]} \otimes \mathcal{L}^{[c t]}$ and quantum on $\hat{A}$. More precisely, $\rho^{\prime}(i), i \in[t]$, $\hat{\Pi}$ can be expressed as

$$
\begin{aligned}
& \left(\rho^{\prime}(i)\right)^{\left(\hat{X}_{[c]}\right)^{t} \hat{A}} \\
& =|\mathcal{L}|^{-c t} \sum_{\mathbf{x}^{t}, \mathbf{l}^{t}} p\left(i ; \mathbf{x}^{t}\right)\left|\mathbf{x}^{t}\right\rangle\left\langle\left.\mathbf{x}^{t}\right|^{\left(X_{[c]}\right)^{\otimes t}} \otimes \mid \mathbf{I}^{t}\right\rangle\left\langle\left.\mathbf{l}^{t}\right|^{\left(\mathcal{L}_{[c]}\right)^{\otimes t}}\right. \\
& \otimes\left(\rho^{\prime}\right)_{\mathbf{x}^{t}(i), \mathbf{I}^{t}(i), \delta}^{A^{\prime}} \otimes(|0\rangle\langle 0|)^{\mathbb{C}^{2}} \otimes(|0\rangle\langle 0|)^{\mathbb{C}^{t+1}}, \\
& (\hat{\Pi})^{\left(\hat{X}_{[c]}\right)^{t} \hat{A}} \\
& =\sum_{\mathbf{x}^{t}, \mathbf{I}^{t}}\left|\mathbf{x}^{t}\right\rangle\left\langle\left.\mathbf{x}^{t}\right|^{\left(X_{[c]}\right)^{\otimes t}} \otimes \mid \mathbf{I}^{t}\right\rangle\left\langle\left.\mathbf{l}^{t}\right|^{\left(\mathcal{L}_{[c]}\right)^{\otimes t}} \otimes(\hat{\Pi})_{\mathbf{x}^{t}, \mathbf{I}^{t}, \delta}^{\hat{A}},\right.
\end{aligned}
$$

where $\left(\rho^{\prime}\right)_{\mathbf{x}, \mathbf{l}, \delta}^{A^{\prime}}$ are quantum states for all computational basis vectors $\mathbf{x} \in \mathcal{X}^{\otimes[c]}, \mathbf{l} \in \mathcal{L}^{\otimes[c]}$ and $(\hat{\Pi})_{\mathbf{x}^{t}, \mathbf{I}^{t}, \delta}^{\hat{A}}$ are POVM elements for all computational basis vectors $\mathbf{x}^{t} \in \mathcal{X}^{\otimes[c t]}, \mathbf{l}^{t} \in \mathcal{L}^{\otimes[c t]}$

2. For all $i \in[t]$,

$$
\begin{aligned}
& \|\left(\rho^{\prime}(i)\right)^{\left(\hat{X}_{[c]}\right)^{t} \hat{A}} \\
& -(\rho(i))^{\left(X_{[c]}\right)^{t} A} \otimes(|0\rangle\langle 0|)^{\mathbb{C}^{2}} \otimes \frac{\mathbb{1}^{\mathcal{L}^{8 c t}}}{|\mathcal{L}|^{c t}} \\
& \otimes(|0\rangle\langle 0|)^{\mathbb{C}^{2}} \otimes(|0\rangle\langle 0|)^{\mathbb{C}^{+1}} \|_{1} \\
& \leq \quad 2^{\frac{c+1}{2}+1} \delta ;
\end{aligned}
$$

3. For all $i \in[t]$,

$$
\operatorname{Tr}\left[(\hat{\Pi})^{\left(\hat{X}_{[c]}\right)^{t} \hat{A}}\left(\rho^{\prime}(i)\right)^{\left(\hat{X}_{[c]}\right)^{t} \hat{A}}\right] \geq 1-\delta^{-2} 2^{2^{c+5}} 3^{c} \epsilon-2^{\frac{c+1}{2}+1} \delta-\alpha ;
$$

4. Let $S \subseteq[c]$. Let $\mathbf{x}_{S}, \mathbf{l}_{S}$ be computational basis vectors in $\mathcal{X}^{\otimes S}, \mathcal{L}^{\otimes S}$. In the following definition, let $\mathbf{x}_{\bar{S}}^{\prime}, \mathbf{l}_{\bar{S}}^{\prime}$ range over all computational basis vectors of $\mathcal{X}^{\otimes([c] \backslash S)}$, $\mathcal{L}^{\otimes([c] \backslash S)}$. For $S \neq\{\}$, define states in $A^{\prime}$,

$$
\left(\rho^{\prime}\right)_{\mathbf{x}_{S}, \mathbf{l}_{S}, \delta}^{A^{\prime}}:=|\mathcal{L}|^{-|\bar{S}|} \sum_{\mathbf{x}_{\bar{S}}^{\prime}, \mathbf{l}_{\bar{S}}^{\prime}} p\left(\mathbf{x}_{\bar{S}}^{\prime} \mid \mathbf{x}_{S}\right)\left(\rho^{\prime}\right)_{\mathbf{x}_{S} \mathbf{x}_{\tilde{S}}^{\prime}, \mathbf{l}_{S} \mathbf{l}_{\tilde{S}}^{\prime}, \delta}^{\prime} .
$$

Analogously define

$$
\rho_{\mathbf{x}_{S}}^{A}:=\sum_{\mathbf{x}_{\bar{S}}^{\prime}} p\left(\mathbf{x}_{\bar{S}}^{\prime} \mid \mathbf{x}_{S}\right) \rho_{\mathbf{x}_{S} \mathbf{x}_{\bar{S}}^{\prime}}^{A} .
$$

Let $\sigma^{A}$ be a fixed quantum state in $A$. For $S=\{\}$, define

$$
\left(\rho^{\prime}\right)_{\{\},\{\}, \delta}^{A^{\prime}}:=\sigma^{A} \otimes|0\rangle\left\langle\left. 0\right|^{\mathbb{C}^{2}}, \quad \rho_{\{\}}^{A}:=\sigma^{A} .\right.
$$

For $i \in[t], S \subseteq[c]$, let $q_{i: S}(\cdot)$ be a probability distribution on $\mathbf{x}^{t}$. Define

$$
\begin{aligned}
& \left(\rho^{\prime}\right)_{i ; S}^{\left(\hat{X}_{[c]}\right)^{t} \hat{A}} \\
& :=|\mathcal{L}|^{-c t} \sum_{\mathbf{x}^{t}} q_{i ; S}\left(\mathbf{x}^{t}\right)\left|\mathbf{x}^{t}\right\rangle\left\langle\left.\mathbf{x}^{t}\right|^{X^{\otimes[c]}} \otimes \mid \mathbf{I}^{t}\right\rangle\left\langle\left.\mathbf{l}^{t}\right|^{\mathcal{L}^{\otimes[c t]}}\right. \\
& \otimes\left(\rho^{\prime}\right)_{\mathbf{x}^{t}(i)_{S}, \mathbf{l}^{t}(i)_{S}, \delta}^{A^{\prime}}, \\
& \rho_{i ; S}^{\left(X_{[c]}\right)^{t} A}:=\sum_{\mathbf{x}^{t}} q_{i ; S}\left(\mathbf{x}^{t}\right)\left|\mathbf{x}^{t}\right\rangle\left\langle\left.\mathbf{x}^{t}\right|^{X^{\otimes[c t]}} \otimes \rho_{\mathbf{X}^{t}(i) S}^{A} .\right.
\end{aligned}
$$

Then,

$\operatorname{Tr}\left[(\hat{\Pi})^{\left(\hat{X}_{[c]}\right)^{t} \hat{A}}\left(\rho^{\prime}\right)_{i ; S}^{\left(\hat{X}_{[c]}\right)^{t} \hat{A}}\right] \leq \frac{1-\alpha}{\alpha} \sum_{j=1}^{t} 2^{-D_{H}^{\epsilon}\left(\rho(j)^{\left(X_{[c]}\right)^{t} A} \| \rho_{i ; S}^{\left(X_{[c]}\right)^{t} A}\right)}$

We now state our main technical proposition.

Proposition 4 Let $\mathcal{H}, \mathcal{L}$ be Hilbert spaces and $\mathcal{X}$ be a finite set. We will also use $\mathcal{X}$ to denote the Hilbert space with computational basis elements indexed by the set $\mathcal{X}$. Let $c$ be a non-negative and $k$ a positive integer. Let $A_{1} \cdots A_{k}$ be a k-partite system where each $A_{i}$ is isomorphic to $\mathcal{H}$. For every $\mathbf{x} \in \mathcal{X}^{c}$, let $\rho_{\mathbf{x}}$ be a quantum state in $A_{[k]}$. Consider the augmented $k$-partite system $A_{1}^{\prime \prime} \cdots A_{k}^{\prime \prime}$ where each $A_{i}^{\prime \prime}$ is isomorphic to

$$
\left(\mathcal{H} \otimes \mathbb{C}^{2}\right) \oplus \bigoplus_{S: i \in S \subseteq[c] \cup[k]}\left(\mathcal{H} \otimes \mathbb{C}^{2}\right) \otimes \mathcal{L}^{\otimes|S|} .
$$

View $\rho_{\mathbf{x}}^{A_{[k]}} \otimes(|0\rangle\langle 0|)^{\left(\mathbb{C}^{2}\right)^{\otimes k}}$ as a state in $A_{[k]}^{\prime \prime}$ under the natural embedding viz. the embedding in the ith system is into the first summand of $A_{i}^{\prime \prime}$ defined above.

Below, $\mathbf{x}, \mathbf{l}$ denote computational basis vectors of $\mathcal{X}^{[c]}$, $\mathcal{L}^{\otimes([c] \cup[k])}$. Let $0 \leq \delta \leq 1$. For each $\mathbf{x} \in \mathcal{X}^{c}$ and each pseudosubpartition $\left(S_{1}, \ldots, S_{l}\right) \vdash \vdash[c] \cup[k]$, let $0 \leq \epsilon_{\mathbf{x},\left(S_{1}, \ldots, S_{l}\right)} \leq 1$. Let $\epsilon_{\mathbf{x}}:=\sum_{\left(S_{1}, \ldots, S_{l}\right)+\vdash[c] \cup[k]} \epsilon_{\mathbf{x},\left(S_{1}, \ldots, S_{l}\right)}$. Then there exist:

- States $\rho_{\mathbf{x}, \mathbf{l}, \delta}^{\prime}$ in $A_{[k]}^{\prime \prime}$ for every $\mathbf{x}, \mathbf{l}$;

- POVM elements $\Pi_{\mathbf{x}, \mathbf{l}, \delta}^{\prime}$ in $A_{[k]}^{\prime \prime}$ for every $\mathbf{x}, \mathbf{l}$;

- For every $\mathbf{l}$ and every $\left(S_{1}, \ldots, S_{l}\right) \vdash \vdash[c] \cup[k], l>0$, numbers $0 \leq \alpha_{\left(S_{1}, \ldots, S_{l}\right), \delta}, \beta_{\left(S_{1}, \ldots, S_{l}\right), \delta} \leq 1$ and isometric embeddings $\mathcal{T}_{\left(S_{1}, \ldots, S_{l}\right), \mathbf{l}, \delta}$ of $\left(\mathcal{H} \otimes \mathbb{C}^{2}\right)^{\otimes k}$ into $A_{[k]}^{\prime \prime}$;

- For every $\mathbf{x}, \mathbf{l}$ and every $\left(S_{1}, \ldots, S_{l}\right) \vdash \vdash[c] \cup[k], l>0$, quantum states $M_{\left(S_{1}, \ldots, S_{l}\right) \mathbf{x}, \mathbf{l}, \delta}$ and unit trace Hermitian operators $N_{\left(S_{1}, \ldots, S_{l}\right), \mathbf{x}, \mathbf{l}, \delta}$ in $A_{[k]}^{\prime \prime}$;

such that:

1. $\left\|\left(\Pi^{\prime}\right)_{\mathbf{x}, \mathbf{l}, \delta}^{A_{[k]}^{\prime \prime}}\right\|_{1} \leq(2|\mathcal{H}|)^{k}$

2. $\left\|M_{\left(S_{1}, \ldots, S_{l}\right), \mathbf{x}, \mathbf{l}, \delta}^{A_{(k]}^{\prime \prime}}\right\|_{\infty} \leq \frac{1}{|\mathcal{L}|}, \beta_{\left(S_{1}, \ldots, S_{l}\right), \delta}\left\|N_{\left(S_{1}, \ldots, S_{l}\right), \mathbf{x}, \mathbf{l}, \delta}^{A_{[k]}^{\prime \prime}}\right\|_{\infty} \leq$ $\frac{3}{\sqrt{|\mathcal{L}|}}$

3. $\left\|\left(\rho_{\mathbf{x}, \mathbf{l}, \delta}^{\prime}\right)^{A_{[k]}^{\prime \prime}}-\rho_{\mathbf{x}}^{A_{[k]}} \otimes\left(|0\rangle\left\langle\left. 0\right|^{\mathbb{C}^{2}}\right)^{\otimes k} \|_{1} \leq 2^{\frac{k+c}{2}+1} \delta\right.\right.$; 
4. $\operatorname{Tr}\left[\left(\Pi_{\mathbf{x}, \mathbf{l}, \delta}^{\prime}\right)^{A_{[k]}^{\prime \prime}}\left(\rho^{A_{[k]}} \otimes\left(|0\rangle\left\langle\left. 0\right|^{\mathbb{C}^{2}}\right)^{\otimes k}\right)\right] \geq 1-\delta^{-2 k} 2^{2^{c k+4}(k+1)^{k}} \epsilon_{\mathbf{x}} ;\right.$

5. Let $\left(S_{1}, \ldots, S_{l}\right) \vdash \vdash[c] \cup[k], l>0$. Define $T:=[k] \backslash$ $\left(S_{1} \cup \cdots \cup S_{l}\right)$. Let $\sigma_{\mathbf{x}}^{A_{T}}$ be a state in $A_{T}$. Let $S \subseteq[c] \cup$ $[k], S \cap[k] \neq\{\}$, Let $\mathbf{x}_{[c] \cap S}, \mathbf{l}_{S}$ be computational basis vectors in $\mathcal{X}^{\otimes([c] \cap S}, \mathcal{L}^{\otimes S}$. Let $p_{[c] \backslash S}$ be a probability distribution on $\mathcal{X}^{\otimes([c] \backslash S)}$. In the following definition, let $\mathbf{x}_{[c] \backslash S}^{\prime}, \mathbf{l}_{\bar{S}}^{\prime}$ range over all computational basis vectors of $\mathcal{X}^{\otimes([c] \backslash S)}, \mathcal{L}^{\otimes \bar{S}}$. Define a state in $A_{S \cap[k]}^{\prime \prime}$,

$\left(\rho^{\prime}\right)_{\mathbf{x}_{S \cap[c]}, \mathbf{l}_{S}, \delta}^{A_{S \cap[k]}^{\prime \prime}}$

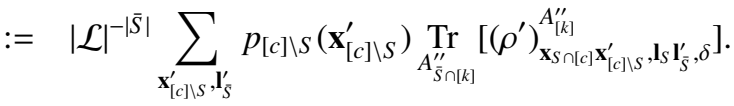

Analogously define

$$
\rho_{\mathbf{x}_{S \cap[c]}}^{A_{S \cap[k]}}:=\sum_{\mathbf{x}_{[c] \backslash S}^{\prime}} p_{[c] \backslash S}\left(\mathbf{x}_{[c] \backslash S}^{\prime}\right) \underset{A_{\tilde{S} \cap[k]}}{\operatorname{Tr}}\left[\rho_{\mathbf{x}_{S \cap[c]} \mathbf{x}_{[c] \backslash S}^{\prime}}^{A_{[k]}}\right] .
$$

Define

$$
\begin{aligned}
\left(\rho^{\prime}\right)_{\mathbf{x}, \mathbf{l},\left(S_{1}, \ldots, S_{l}\right), \delta}^{A_{[k]}^{\prime \prime}} & \\
:= & \left(\rho_{\mathbf{x}_{S_{1} \cap[c]}^{\prime}, \mathbf{l}_{S_{1}, \delta}}^{\prime}\right)^{A_{S_{1} \cap[k]}^{\prime \prime}} \\
& \otimes \cdots \otimes \\
& \left(\rho_{\mathbf{x}_{\left.S_{l} \cap[c]\right)}^{\prime}, \mathbf{l}_{S_{l}, \delta}}^{\prime}\right)^{A_{S_{l} \cap[k]}^{\prime \prime}} \\
& \otimes\left(\sigma_{\mathbf{x}}^{A_{T}} \otimes\left(|0\rangle\left\langle\left. 0\right|^{\mathbb{C}^{2}}\right)^{\otimes[T]}\right),\right. \\
\rho_{\mathbf{x},\left(S_{1}, \ldots, S_{l}\right)}^{A_{[k]}}:= & \rho_{\mathbf{x}_{S_{1} \cap[c]}}^{A_{S_{1} \cap[k]}} \otimes \cdots \otimes \rho_{\mathbf{x}_{S_{l} \cap[c]}}^{A_{S_{l} \cap[k]}} \otimes \sigma_{\mathbf{x}}^{A_{T}} .
\end{aligned}
$$

Then,

$$
\begin{aligned}
& \left(\rho^{\prime}\right)_{\mathbf{x}, \mathbf{l},\left(S_{1}, \ldots, S_{l}\right), \delta}^{A_{[k]}^{\prime \prime}} \\
& =\quad \alpha_{\left(S_{1}, \ldots, S_{l}\right), \delta}\left(\mathcal{T}_{\left(S_{1}, \ldots S_{l}\right), \mathbf{l}, \delta}\left(\rho_{\mathbf{x},\left(S_{1}, \ldots, S_{l}\right)}^{A_{[k]}} \otimes\left(|0\rangle\left\langle\left. 0\right|^{\mathbb{C}^{2}}\right)^{\otimes k}\right)\right)^{A_{[k]}^{\prime \prime}}\right. \\
& +\beta_{\left(S_{1}, \ldots, S_{l}\right), \delta} N_{\left(S_{1}, \ldots S_{l}\right), \mathbf{x}, \mathbf{l}, \delta}^{A_{[k]}^{\prime \prime}} \\
& +\left(1-\alpha_{\left(S_{1}, \ldots, S_{l}\right), \delta}-\beta_{\left(S_{1}, \ldots, S_{l}\right), \delta}\right) M_{\left(S_{1}, \ldots S_{l}\right), \mathbf{x}, \mathbf{l}, \delta}^{A_{[k}^{\prime \prime}}
\end{aligned}
$$

Moreover, the support of $M_{\left(S_{1}, \ldots S_{l}\right), \mathbf{x}, \mathbf{l}, \delta}^{A_{[k]}^{\prime \prime}}$ is orthogonal to the support of the sum of the first two terms in the above equation, and $\beta_{\left(S_{1}, \ldots, S_{l}\right), \delta}=0$ if $c=0$ or $[c] \subseteq S_{i}$ for some $i \in[l]$;

6. $\operatorname{Tr}\left[\left(\Pi^{\prime}\right)_{\mathbf{x}, \mathbf{l}, \delta}^{A_{[k]}^{\prime \prime}}\left(\mathcal{T}_{\left(S_{1}, \ldots S_{l}\right), \mathbf{l}, \delta}\left(\rho_{\mathbf{x},\left(S_{1}, \ldots, S_{l}\right)}^{A_{[k]}} \otimes\left(|0\rangle\left\langle\left. 0\right|^{\mathbb{C}^{2}}\right)^{\otimes k}\right)\right)^{\left.A_{[k]}^{\prime \prime}\right]} \leq\right.\right.$ $2^{-D_{H}^{\epsilon_{\mathbf{x},\left(S_{1}, \ldots, S_{l}\right)}}\left(\rho_{\mathbf{x}}^{A_{[k]}} \| \rho_{\mathbf{x},\left(S_{1}, \ldots, S_{l}\right)}^{A_{[k}}\right)}$

Proof: Proved in Appendix I.

\section{One-shot inner bound for the classical quantum MAC}

In this section, we prove our general one-shot inner bound for the cq-MAC for any number of senders. We illustrate our method by considering two senders only, but the method works for any number of senders. We will use Corollary 4 as our main technical workhorse in order to obtain the inner bound. The difference between the inner bound in this section and the inner bound in Section 4 is that we use a socalled 'time-sharing' random variable $U$ now.

Define a new sample space $\mathcal{U}$ and put on it a probability distribution $p(u)$. The resulting random variable $U$ plays the role, in the one-shot setting, of the so-called 'time sharing random variable' familiar from classical iid network information theory. Fix conditional probability distributions $p(x \mid u), p(y \mid u)$ on sets $\mathcal{X}, \mathcal{Y}$. Consider the classical-quantum state

$$
\rho^{U X Y Z}:=\sum_{u, x, y} p(u) p(x \mid u) p(y \mid u)|u, x, y\rangle\left\langle u, x,\left.y\right|^{U X Y} \otimes \rho_{x y}^{Z} .\right.
$$

This state 'controls' the encoding and decoding performance for the channel $\mathfrak{C}$.

Consider a new alphabet, as well as Hilbert space, $\mathcal{L}$ and define the augmented systems $\mathcal{U}^{\prime}:=\mathcal{U} \otimes \mathcal{L}, X^{\prime}:=\mathcal{X} \otimes \mathcal{L}$, $\mathcal{Y}^{\prime}:=\mathcal{Y} \otimes \mathcal{L}$, and the extended system $\mathcal{Z}^{\prime}$ defined in Corollary 4 with $c=3$. Consider a 'variant' cq-MAC $\mathfrak{C}^{\prime}$ with input alphabets $\mathcal{U}^{\prime}, \mathcal{X}^{\prime}, \boldsymbol{Y}^{\prime}$ and output Hilbert space $\mathcal{Z}^{\prime}$. On input $\left(u, l_{u}\right),\left(x, l_{x}\right),\left(y, l_{y}\right)$ to $\mathbb{C}^{\prime}$, the output of $\mathbb{C}^{\prime}$ is the state $\rho_{x, y}^{Z} \otimes|0\rangle\left\langle\left. 0\right|^{\mathbb{C}^{2}}\right.$. The output of $\mathfrak{C}^{\prime}$ is taken to embed into the first summand in the definition of $\mathcal{Z}^{\prime}$ in Corollary 4 The classical quantum state 'controlling' the encoding and decoding for $\mathbb{C}^{\prime}$ is nothing but $\rho^{U X Y Z} \otimes|0\rangle\left\langle\left. 0\right|^{\mathbb{C}^{2}} \otimes \frac{\mathbb{1}^{\mathcal{L}^{\otimes 3}}}{|\mathcal{L}|^{3}}\right.$. The channel $\mathfrak{C}^{\prime}$ can be trivally obtained from channel $\mathfrak{C}^{2}$. The expected average decoding error for $\mathbb{C}^{\prime}$ is the same as the expected average decoding error for $\mathbb{C}$ for the same rate pair $\left(R_{1}, R_{2}\right)$. In fact, an encoding-decoding scheme for $\mathbb{C}^{\prime}$ immediately gives an encoding-decoding scheme for $\mathfrak{C}$ with the same rate pair $\left(R_{1}, R_{2}\right)$ and the same decoding error.

Let $0 \leq \delta \leq 1$. Next, consider a 'perturbed' cq-MAC $\mathfrak{C}^{\prime \prime}$ with the same input alphabets and output Hilbert space as in $\mathfrak{C}^{\prime}$. However, on input $\left(u, l_{u}\right),\left(x, l_{x}\right),\left(y, l_{y}\right)$ the output is the state $\left(\rho^{\prime}\right)_{\left(u, l_{u}\right),\left(x, l_{x}\right),\left(y, l_{y}\right)}^{Z^{\prime}}$ provided by setting $c=3$ in Corollay 4 . Consider the classical quantum state

$$
\begin{gathered}
\left(\rho^{\prime}\right)^{U^{\prime} X^{\prime} Y^{\prime} Z^{\prime}} \\
:=|\mathcal{L}|^{-3} \sum_{u, x, y, l_{u}, l_{x}, l_{y}} p(u) p(x \mid u) p(y \mid u)\left|u, l_{u}\right\rangle\left\langle u,\left.l_{u}\right|^{U^{\prime}}\right. \\
\otimes\left|x, l_{x}\right\rangle\left\langle x,\left.l_{x}\right|^{X^{\prime}} \otimes \mid y, l_{y}\right\rangle\left\langle y,\left.l_{y}\right|^{Y^{\prime}}\right. \\
\otimes\left(\rho^{\prime}\right)_{u, x, y, l_{u}, l_{x} l_{y}, \delta} .
\end{gathered}
$$

This state 'controls' the encoding and decoding performance for the channel $\mathfrak{C}^{\prime \prime}$. By Claim 2 of Corollary 4

$$
\|\left(\rho^{\prime}\right)^{U^{\prime} X^{\prime} Y^{\prime} Z^{\prime}}-\rho^{X Y Z} \otimes|0\rangle\left\langle\left. 0\right|^{\left(\mathbb{C}^{2}\right)^{\otimes 3}} \otimes \frac{\mathbb{1}^{\mathcal{L}^{\otimes 3}}}{|\mathcal{L}|^{3}} \|_{1} \leq 2^{3} \delta .\right.
$$

Thus, the expected average decoding error for $\mathfrak{C}^{\prime \prime}$ is at most the expected average decoding error for $\mathfrak{C}^{\prime}$, which is also the same as the expected average decoding error for $\mathfrak{C}$, plus $2^{3} \delta$, for the same rate pair $\left(R_{1}, R_{2}\right)$, and the same decoding strategy.

Consider the following randomised construction of a codebook $C$ for Alice and Bob for communication over the channel $\mathbb{C}^{\prime \prime}$. Fix probability distributions $p(u), p(x \mid u), p(y \mid u)$ on 
sets $\mathcal{U}, \mathcal{X}, \mathcal{Y}$. Choose a sample $\left(u, l_{u}\right)$ according to the product of the distribution $p(u)$ on $\mathcal{U}$ and the uniform distribution on computational basis vectors of $\mathcal{L}$. For all $m_{1} \in\left[2^{R_{1}}\right]$, choose $\left(x, l_{x}\right)\left(m_{1}\right) \in \mathcal{X} \times \mathcal{L}$ independently according to the product of the distribution $p(x \mid u)$ on $\mathcal{X}$ and the uniform distribution on $\mathcal{L}$. Similarly for all $m_{2} \in\left[2^{R_{2}}\right]$, choose $\left(y, l_{y}\right)\left(m_{2}\right) \in$ $\mathcal{Y} \times \mathcal{L}$ independently according to the product of the distribution $p(y \mid u)$ on $\mathcal{Y}$ and the uniform distribution on $\mathcal{L}$.

We now describe the decoding strategy that Charlie follows in order to try and guess the message pair $\left(m_{1}, m_{2}\right)$ that was actually sent, given the output of $\mathfrak{C}^{\prime \prime}$. Let $\left(\Pi^{\prime}\right)_{u, x, y, l_{u}, l_{x}, l_{y}, \delta}^{Z^{\prime}}$ be the POVM elements provided by Corollary 4. Charlie uses the pretty good measurement constructed from the POVM elements $\left(\Pi^{\prime}\right)_{\left(u, l_{u}\right),\left(x, l_{x}\right)\left(m_{1}\right),\left(y, l_{y}\right)\left(m_{2}\right), \delta}^{Z^{\prime}}$, where $\left(m_{1}, m_{2}\right) \times\left[2^{R_{1}}\right] \times$ $\left[2^{R_{2}}\right]$. We now analyse the expectation, under the choice of a random codebook $C$, of the error probability of Charlie's decoding algorithm. Suppose the message pair $\left(m_{1}, m_{2}\right)$ is inputted to $\mathfrak{C}^{\prime \prime}$. The output of $\mathfrak{C}^{\prime \prime}$ is the state $\left(\rho^{\prime}\right)_{\left(u, l_{u}\right),\left(x, l_{x}\right)\left(m_{1}\right),\left(y, l_{y}\right)\left(m_{2}\right)}^{Z^{\prime}}$. Let $\Lambda_{\hat{m}_{1}, \hat{m}_{2}}^{Z^{\prime}}$ be the POVM element corresponding to decoded output $\left(\hat{m}_{1}, \hat{m}_{2}\right)$ arising from the pretty good measurement. By the Hayashi-Nagaoka inequality [14], the decoding error for $\left(m_{1}, m_{2}\right)$ is upper bounded by

$$
\begin{aligned}
& \operatorname{Tr}\left[\left(\mathbf{1}^{Z^{\prime}}-\Lambda_{m_{1}, m_{2}}^{Z^{\prime}}\right)\left(\rho^{\prime}\right)_{\left(u, l_{u}\right),\left(x, l_{x}\right)\left(m_{1}\right),\left(y, l_{y}\right)\left(m_{2}\right)}^{Z^{\prime}}\right] \\
& \leq 2 \operatorname{Tr}\left[\left(\mathbb{1}^{Z^{\prime}}-\left(\Pi^{\prime}\right)_{\left(u, l_{u}\right),\left(x, l_{x}\right)\left(m_{1}\right),\left(y, l_{y}\right)\left(m_{2}\right)}^{Z^{\prime}}\right)\right. \\
& \left.\left(\rho^{\prime}\right)_{\left(u, l_{u}\right),\left(x, l_{x}\right)\left(m_{1}\right),\left(y, l_{y}\right)\left(m_{2}\right)}^{Z^{\prime}}\right] \\
& +4 \sum_{\left(\hat{m}_{1}, \hat{m}_{2}\right) \neq\left(m_{1}, m_{2}\right)} \operatorname{Tr}\left[\left(\Pi^{\prime}\right)_{\left(u, l_{u}\right),\left(x, l_{x}\right)\left(\hat{m}_{1}\right),\left(y, l_{y}\right)\left(\hat{m}_{2}\right)}^{Z^{\prime}}\right. \\
& \left.\left(\rho^{\prime}\right)_{\left(u, l_{u}\right),\left(x, l_{x}\right)\left(m_{1}\right),\left(y, l_{y}\right)\left(m_{2}\right)}^{Z^{\prime}}\right] \\
& =2 \operatorname{Tr}\left[\left(\mathbb{1}^{Z^{\prime}}-\left(\Pi^{\prime}\right)_{\left(u, l_{u}\right),\left(x, l_{x}\right)\left(m_{1}\right),\left(y, l_{y}\right)\left(m_{2}\right)}^{Z^{\prime}}\right)\right. \\
& \left.\left(\rho^{\prime}\right)_{\left(u, l_{u}\right),\left(x, l_{x}\right)\left(m_{1}\right),\left(y, l_{y}\right)\left(m_{2}\right)}^{Z^{\prime}}\right] \\
& +4 \sum_{\hat{m}_{1} \neq m_{1}} \operatorname{Tr}\left[\left(\Pi^{\prime}\right)_{\left(u, l_{u}\right),\left(x, l_{x}\right)\left(\hat{m}_{1}\right),\left(y, l_{y}\right)\left(m_{2}\right)}^{Z^{\prime}}\right. \\
& \left.\left(\rho^{\prime}\right)_{\left(u, l_{u}\right),\left(x, l_{x}\right)\left(m_{1}\right),\left(y, l_{y}\right)\left(m_{2}\right)}^{Z^{\prime}}\right] \\
& +4 \sum_{\hat{m}_{2} \neq m_{2}} \operatorname{Tr}\left[\left(\Pi^{\prime}\right)_{\left(u, l_{u}\right),\left(x, l_{x}\right)\left(m_{1}\right),\left(y, l_{y}\right)\left(\hat{m}_{2}\right)}^{Z^{\prime}}\right. \\
& \left.\left(\rho^{\prime}\right)_{\left(u, l_{u}\right),\left(x, l_{x}\right)\left(m_{1}\right),\left(y, l_{y}\right)\left(m_{2}\right)}^{Z^{\prime}}\right] \\
& +4 \sum_{\hat{m}_{1} \neq m_{1}, \hat{m}_{2} \neq m_{2}} \operatorname{Tr}\left[\left(\Pi^{\prime}\right)_{\left(u, l_{u}\right),\left(x, l_{x}\right)\left(\hat{m}_{1}\right),\left(y, l_{y}\right)\left(\hat{m}_{2}\right)}^{Z^{\prime}}\right. \\
& \left.\left(\rho^{\prime}\right)_{\left(u, l_{u}\right),\left(x, l_{x}\right)\left(m_{1}\right),\left(y, l_{y}\right)\left(m_{2}\right)}^{Z^{\prime}}\right]
\end{aligned}
$$

Define the POVM element

$$
\begin{gathered}
\left(\Pi^{\prime}\right)^{U^{\prime} X^{\prime} Y^{\prime} Z^{\prime}} \\
:=\sum_{u, x, y, l_{u}, l_{x}, l_{y}}\left|u, l_{u}\right\rangle\left\langle u,\left.l_{u}\right|^{U^{\prime}} \otimes \mid x, l_{x}\right\rangle\left\langle x,\left.l_{x}\right|^{X^{\prime}} \otimes \mid y, l_{y}\right\rangle\left\langle y,\left.l_{y}\right|^{Y^{\prime}}\right. \\
\otimes\left(\Pi^{\prime}\right)_{\left(u, l_{u}\right),\left(x, l_{x}\right),\left(y, l_{y}\right), \delta}^{Z^{\prime}}
\end{gathered}
$$

as in Corollary 4 From Corollary 4, recall the hypothesis testing conditional mutual information quantitites $I_{H}^{\epsilon}(X$ : $Z \mid U Y), I_{H}^{\epsilon}(Y: Z \mid U X), I_{H}^{\epsilon}(X Y: Z \mid U)$ calculated with respect to the state $\rho^{U X Y Z}$ corresponding to the original channel $\mathfrak{C}$.
The expectation, over the choice of the random codebook $C$, of the decoding error for $\left(m_{1}, m_{2}\right)$ is upper bounded by

$$
\begin{aligned}
& \underset{C}{\mathbf{E}}\left[\operatorname{Tr}\left[\left(\mathbb{1}^{Z^{\prime}}-\Lambda_{m_{1}, m_{2}}^{Z^{\prime}}\right)\left(\rho^{\prime}\right)_{\left(u, l_{u}\right),\left(x, l_{x}\right)\left(m_{1}\right),\left(y, l_{y}\right)\left(m_{2}\right)}^{Z^{\prime}}\right]\right] \\
& \leq 2 \underset{C}{\mathbf{E}}\left[\operatorname { T r } \left[\left(\mathbb{1}^{Z^{\prime}}-\left(\Pi^{\prime}\right)_{\left(u, l_{u}\right),\left(x, l_{x}\right)\left(m_{1}\right),\left(y, l_{y}\right)\left(m_{2}\right)}^{Z^{\prime}}\right)\right.\right. \\
& \left.\left.\left(\rho^{\prime}\right)_{\left(u, l_{u}\right),\left(x, l_{x}\right)\left(m_{1}\right),\left(y, l_{y}\right)\left(m_{2}\right)}^{Z^{\prime}}\right]\right] \\
& +4 \sum_{\hat{m}_{1} \neq m_{1}} \underset{C}{\mathbf{E}}\left[\operatorname { T r } \left[\left(\Pi^{\prime}\right)_{\left(u, l_{u}\right),\left(x, l_{x}\right)\left(\hat{m}_{1}\right),\left(y, l_{y}\right)\left(m_{2}\right)}^{Z^{\prime}}\right.\right. \\
& \left.\left.\left(\rho^{\prime}\right)_{\left(u, l_{u}\right),\left(x, l_{x}\right)\left(m_{1}\right),\left(y, l_{y}\right)\left(m_{2}\right)}^{Z^{\prime}}\right]\right] \\
& +4 \sum_{\hat{m}_{2} \neq m_{2}} \underset{C}{\mathbf{E}}\left[\operatorname { T r } \left[\left(\Pi^{\prime}\right)_{\left(u, l_{u}\right),\left(x, l_{x}\right)\left(m_{1}\right),\left(y, l_{y}\right)\left(\hat{m}_{2}\right)}^{Z^{\prime}}\right.\right. \\
& \left.\left.\left(\rho^{\prime}\right)_{\left(u, l_{u}\right),\left(x, l_{x}\right)\left(m_{1}\right),\left(y, l_{y}\right)\left(m_{2}\right)}^{Z^{\prime}}\right]\right] \\
& +4 \sum_{\hat{m}_{1} \neq m_{1}, \hat{m}_{2} \neq m_{2}} \underset{C}{\mathbf{E}}\left[\operatorname { T r } \left[\left(\Pi^{\prime}\right)_{\left(u, l_{u}\right),\left(x, l_{x}\right)\left(\hat{m}_{1}\right),\left(y, l_{y}\right)\left(\hat{m}_{2}\right)}^{Z^{\prime}}\right.\right. \\
& \left.\left.\left(\rho^{\prime}\right)_{\left(u, l_{u}\right),\left(x, l_{x}\right)\left(m_{1}\right),\left(y, l_{y}\right)\left(m_{2}\right)}^{Z^{\prime}}\right]\right]
\end{aligned}
$$$$
=2|\mathcal{L}|^{-3} \sum_{u, x, y, l_{u}, l_{x}, l_{y}} p(u) p(x \mid u) p(y \mid u)
$$$$
\operatorname{Tr}\left[\left(\mathbb{1}^{Z^{\prime}}-\left(\Pi^{\prime}\right)_{\left(u, l_{u}\right),\left(x, l_{x}\right),\left(y, l_{y}\right)}^{Z^{\prime}}\right)\right.
$$$$
\left.\left(\rho^{\prime}\right)_{\left(u, l_{u}\right),\left(x, l_{x}\right),\left(y, l_{y}\right)}^{Z^{\prime}}\right]
$$$$
+4\left(2^{R_{1}}-1\right)|\mathcal{L}|^{-4} \sum_{u, l_{u}, x, l_{x}, x^{\prime}, l_{x}^{\prime}, y, l_{y}} p(u) p(x \mid u) p\left(x^{\prime} \mid u\right) p(y \mid u)
$$$$
\left.\operatorname{Tr}\left[\left(\Pi^{\prime}\right)_{\left(u, l_{u}\right),\left(x^{\prime}, l_{x}^{\prime}\right),\left(y, l_{y}\right)}^{Z^{\prime}}\right)\left(\rho^{\prime}\right)_{\left(u, l_{u}\right),\left(x, l_{x}\right),\left(y, l_{y}\right)}^{Z^{\prime}}\right]
$$$$
+4\left(2^{R_{2}}-1\right)|\mathcal{L}|^{-4} \sum_{u, l_{u}, x, l_{x}, x^{\prime}, l_{x}^{\prime}, y, l_{y}} p(u) p(x \mid u) p(y \mid u) p\left(y^{\prime} \mid u\right)
$$$$
\left.\operatorname{Tr}\left[\left(\Pi^{\prime}\right)_{\left(u, l_{u}\right),\left(x, l_{x}\right),\left(y^{\prime}, l_{y}^{\prime}\right)}^{Z^{\prime}}\right)\left(\rho^{\prime}\right)_{\left(u, l_{u}\right),\left(x, l_{x}\right),\left(y, l_{y}\right)}^{Z^{\prime}}\right]
$$$$
+4\left(2^{R_{1}}-1\right)\left(2^{R_{2}}-1\right)|\mathcal{L}|^{-5}
$$$$
\sum_{u, l_{u}, x, l_{x}, x^{\prime}, l_{x}^{\prime}, y, l_{y}, y^{\prime}, l_{y}^{\prime}} p(u) p(x \mid u) p\left(x^{\prime} \mid u\right) p(y \mid u) p\left(y^{\prime} \mid u\right)
$$

$$
\left.\operatorname{Tr}\left[\left(\Pi^{\prime}\right)_{\left(u, l_{u}\right),\left(x^{\prime}, l_{x}^{\prime}\right),\left(y^{\prime}, l_{y}^{\prime}\right)}^{Z^{\prime}}\right)\left(\rho^{\prime}\right)_{\left(u, l_{u}\right),\left(x, l_{x}\right),\left(y, l_{y}\right)}^{Z^{\prime}}\right]
$$$$
=2 \operatorname{Tr}\left[\left(\mathbb{1}^{U^{\prime} X^{\prime} Y^{\prime} Z^{\prime}}-\left(\Pi^{\prime}\right)^{U^{\prime} X^{\prime} Y^{\prime} Z^{\prime}}\right)\left(\rho^{\prime}\right)^{U^{\prime} X^{\prime} Y^{\prime} Z^{\prime}}\right]
$$$$
+4\left(2^{R_{1}}-1\right) \operatorname{Tr}\left[\left(\Pi^{\prime}\right)^{U^{\prime} X^{\prime} Y^{\prime} Z^{\prime}}\left(\rho^{\prime}\right)_{\left(\left\{U^{\prime}\right\},\left\{X^{\prime}\right\},\left\{Y^{\prime}\right\}\right)}^{U^{\prime} X^{\prime} Y^{\prime} Z^{\prime}}\right]
$$$$
+4\left(2^{R_{2}}-1\right) \operatorname{Tr}\left[\left(\Pi^{\prime}\right)^{U^{\prime} X^{\prime} Y^{\prime} Z^{\prime}}\left(\rho^{\prime}\right)_{\left(\left\{U^{\prime}\right\},\left\{Y^{\prime}\right\},\left\{X^{\prime}\right\}\right)}^{U^{\prime} X^{\prime} X^{\prime} Z^{\prime}}\right]
$$$$
+4\left(2^{R_{1}}-1\right)\left(2^{R_{2}}-1\right) \operatorname{Tr}\left[\left(\Pi^{\prime}\right)^{U^{\prime} X^{\prime} Y^{\prime} Z^{\prime}}\left(\rho^{\prime}\right)_{\left(\left\{U^{\prime}\right\},\left\{X^{\prime}, Y^{\prime}\right\},\{\}\right)}^{U^{\prime} X^{\prime} Y^{\prime} Y^{\prime}}\right]
$$$$
\leq 2\left(\delta^{-2} 2^{134} \epsilon+2^{3} \delta\right)+2^{R_{1}+2-I_{H}^{\epsilon}(X: Y Z \mid U)_{\rho}}+2^{R_{2}+2-I_{H}^{\epsilon}(Y: X Z \mid U)_{\rho}}
$$$$
+2^{R_{1}+R_{2}+2-I_{H}^{\epsilon}(X Y: Z \mid U)_{\rho}},
$$

where we used Claims 3, 4 of Corollary 4 in the last inequality above.

Taking $\delta=\epsilon^{1 / 3}$ and choosing a rate pair $\left(R_{1}, R_{2}\right)$ satisfying

$$
\begin{aligned}
R_{1} & \leq I_{H}^{\epsilon}(X: Y Z \mid U)_{\rho}-2-\log \frac{1}{\epsilon}, \\
R_{2} & \leq I_{H}^{\epsilon}(Y: X Z \mid U)_{\rho}-2-\log \frac{1}{\epsilon}, \\
R_{1}+R_{2} & \leq I_{H}^{\epsilon}(X Y: Z \mid U)_{\rho}-2-\log \frac{1}{\epsilon},
\end{aligned}
$$


ensures that the expected average decoding error for channel $\mathfrak{C}^{\prime \prime}$ is at most $2^{135} \epsilon^{1 / 3}$. This implies that the expected average decoding error for the original channel $\mathbb{C}$ is at most $2^{135} \epsilon^{1 / 3}$. Thus there exists a codebook $C$ with average decoding error for $\mathbb{C}$ at most $2^{135} \epsilon^{1 / 3}$. By a standard technique of taking maps from classical symbols to arbitrary quantum states, we can then prove the following theorem.

Theorem 2 Let $\mathfrak{C}: X^{\prime} Y^{\prime} \rightarrow Z$ be a quantum multiple access channel. Let $\mathcal{U}, \mathcal{X}, \mathcal{Y}$ be three new sample spaces. For every element $x \in \mathcal{X}$, let $\sigma_{x}^{X^{\prime}}$ be a quantum state in the input Hilbert space $X^{\prime}$ of $\mathfrak{C}$. Similarly, for every element $y \in \mathcal{Y}$, let $\sigma_{y}^{Y^{\prime}}$ be a quantum state in the input Hilbert space $Y^{\prime}$ of $\mathfrak{C}$. Let $p(u) p(x \mid u) p(y \mid u)$ be a probability distribution on $\mathcal{U} \times \mathcal{X} \times \mathcal{Y}$. Consider the classical quantum state

$$
\begin{gathered}
\rho^{U X Y Z}:=\sum_{u, x, y} p(u) p(x \mid u) p(y \mid u)|u, x, y\rangle\left\langle u, x,\left.y\right|^{U X Y}\right. \\
\otimes\left(\mathfrak{C}\left(\sigma_{x}^{X^{\prime}} \otimes \sigma_{y}^{Y^{\prime}}\right)\right)^{Z} .
\end{gathered}
$$

Let $R_{1}, R_{2}, \epsilon$, be such that

$$
\begin{aligned}
R_{1} & \leq I_{H}^{\epsilon}(X: Y Z \mid U)_{\rho}-2-\log \frac{1}{\epsilon}, \\
R_{2} & \leq I_{H}^{\epsilon}(Y: X Z \mid U)_{\rho}-2-\log \frac{1}{\epsilon}, \\
R_{1}+R_{2} & \leq I_{H}^{\epsilon}(X Y: Z \mid U)_{\rho}-2-\log \frac{1}{\epsilon} .
\end{aligned}
$$

Then there exists an $\left(R_{1}, R_{2}, 2^{135} \epsilon^{1 / 3}\right)$-quantum MAC code for sending classical information through $\mathfrak{C}$.

A similar bound can be proved for sending classical information through an entanglement assisted q-MAC by using the position based coding technique of Anshu, Jain and Warsi [29]. Earlier, Qi, Wang and Wilde [19] had constructed a one-shot simultaneous decoder for this problem which also used position based coding, but their inner bound is suboptimal when reduced to the asymptotic iid setting. In contrast, our one-shot inner bound reduces to the best known inner bound in the asymptotic iid setting which was proved by Hsieh, Devetak and Winter [30] using successive cancellation arguments.

Theorem 3 Let $\mathfrak{c}: X^{\prime} Y^{\prime} \rightarrow Z$ be a quantum multiple access channel. Let $\mathcal{U}, \mathcal{X}, \mathcal{Y}$ be three new Hilbert spaces. Let $\psi^{U X Y X^{\prime} Y^{\prime}}$ be a classical quantum state with the following structure:

$$
\psi^{U X Y X^{\prime} Y^{\prime}}=\sum_{u} p(u)|u\rangle\left\langle\left. u\right|^{U} \otimes \psi_{u}^{X X^{\prime}} \otimes \psi_{u}^{Y Y^{\prime}}\right.
$$

\section{Consider the classical quantum state}

$$
\rho^{U X Y Z}:=\sum_{u} p(u)|u\rangle\left\langle\left. u\right|^{U} \otimes\left(\mathbb{C}^{X^{\prime} Y^{\prime} \rightarrow Z}\left(\psi_{u}^{X X^{\prime}} \otimes \psi_{u}^{Y Y^{\prime}}\right)\right)^{X Y Z} .\right.
$$

Let $R_{1}, R_{2}, \epsilon$, be such that

$$
R_{1} \leq I_{H}^{\epsilon}(X: Y Z \mid U)_{\rho}-2-\log \frac{1}{\epsilon},
$$

$$
\begin{aligned}
R_{2} & \leq I_{H}^{\epsilon}(Y: X Z \mid U)_{\rho}-2-\log \frac{1}{\epsilon}, \\
R_{1}+R_{2} & \leq I_{H}^{\epsilon}(X Y: Z \mid U)_{\rho}-2-\log \frac{1}{\epsilon} .
\end{aligned}
$$

Then there exists an entanglement assisted $\left(R_{1}, R_{2}, 2^{135} \epsilon^{1 / 3}\right)$ quantum MAC code for sending classical information through c.

\section{Conclusion and open problems}

In this work, we have proved a one-shot classical quantum joint typicality lemma that not only extends the iid classical conditional joint typicality lemma to the one-shot and quantum settings, but also extends intersection and union arguments that are ubiquitous in classical network information theory to the quantum setting. We introducted two novel tools in the process of proving our joint typicality lemma viz. tilting, and smoothing and augmentation, which should be useful elsewhere. Our lemma allows us to transport many packing arguments arising in proofs of inner bounds from the classical to the quantum setting. We illustrated this by constructing a simultaneous decoder for the one-shot classical quantum MAC. More of such applications can be found in the companion paper [11].

However, the statement of our quantum joint typicality lemma is not as strong as the classical statement. It can only handle negative hypothesis states that are a tensor product of marginals and at most one arbitrary quantum state. Proving a quantum lemma that can handle arbitrary negative hypothesis states, as in the classical setting, is an important open problem.

Though the main advance of our quantum joint typicality lemma is a robust notion of intersection of projectors, there are some technical issues which prevent us from proving a chain rule for hypothesis testing mutual information which an ideal notion of intersection of projectors would have allowed one to do. The main issue here is that the smoothing and augmentation procedure ensures that tensor products of marginals corresponding to different partitions of a multipartite quantum state essentially lie in 'well-separated' subspaces. This feature which was crucial for proving our joint typicality lemma turns out to be a bottleneck for proving a chain rule. A similar bottleneck arises if one were to attempt to prove the simultaneous smoothing conjecture using our techniques.

Another drawback of our quantum lemma is that it cannot handle covering arguments and their unions and intersections that often arise in source coding applications. This is unlike the classical case. Again this issue seems to be related to the simultaneous smoothing conjecture and the bottleneck mentioned above. Addressing this deficiency is another important open problem.

\section{Acknowledgements}

I profusely thank Mark Wilde for pointing out the need for a simultaneous decoder for the quantum multiple access chan- 
nel many years back while I was visitng McGill, and underscoring its importance for quantum network information theory. He also provided pointers to several important references. Patrick Hayden, David Ding and Hrant Gharibyan took great pains in reading a preliminary draft of this paper and provided useful feedback. I thank Rahul Jain for encouraging me to write up this result in the face of a long delay. I thank an anonymous referee for suggesting to give a full self contained solution for the pathological example of failure of 'union by span' in the introduction, and more detailed intuition behind smoothing and augmentation. I am grateful to three anonymous referees for suggesting to give a self-contained proof of the 'baby case' of 'intersection' of two projectors / two sender classical quantum multiple access channel. I thank Naqueeb Warsi for pointing me to his paper on the entanglement assisted compound quantum channel and suggesting a possible application of tilted span of projectors to that problem. Finally, I thank an anonymous referee who pointed out a possible application of our tilted span to the 'quantum OR bound' of Harrow, Lin and Montanaro and asked whether our notion of intersection of projectors can lead to the proof of a chain rule for one shot mutual information quantities.

\section{References}

[1] A. El Gamal and Y-H. Kim. Network Information Theory. Cambridge University Press, 2012.

[2] C. Shannon. A mathematical theory of communication. Bell System Technical Journal, 27:379-423, 623-656, 1948.

[3] D. Deutsch. Quantum theory, the Church-Turing principle and the universal quantum computer. Proceedings of the Royal Society of London Series A, 400:97-117, 1985.

[4] A. Holevo. Bounds for the quantity of information transmitted by a quantum communication channel. Problems of Information Transmission, 9:177-183, 1973.

[5] P Shor. Polynomial-time algorithms for prime factorization and discrete logarithms on a quantum computer. SIAM Journal on Computing, 26:1484-1509, 1997.

[6] A. Holevo. The capacity of the quantum channel with general signal states. IEEE Transactions on Information Theory, 44:269-273, 1998.

[7] B. Schumacher and M. Westmoreland. Sending classical information via noisy quantum channels. Physical Review A, 56:131-138, 1997.

[8] L. Wang and R. Renner. One-shot classical-quantum capacity and hypothesis testing. Physical Review Letters, 108:2005:12005:5, 2012.

[9] A. Winter. The capacity of the quantum multiple-access channel. IEEE Transactions on Information Theory, 47:30593065, 2001.

[10] P. Sen. Achieving the Han-Kobayashi inner bound for the quantum interference channel. In IEEE International Symposium on Information Theory (ISIT), pages 736-740, 2012. Full version at arXiv:1109.0802.
[11] P. Sen. Inner bounds via simultaneous decoding in quantum network information theory. Available at arXiv:1806.07276, 2018.

[12] V. Belavkin. Optimal multiple quantum statistical hypothesis testing. Stochastics, 1:315-345, 1975.

[13] V. Belavkin. . Radiotekhnika i Electronika, 20:1177-1185, 1975. English translation: "Optimal distinction of nonorthogonal quantum signals", Radio Engineering and Electronic Physics, 20, pp. 39-47, 1975.

[14] M. Hayashi and H. Nagaoka. General formulas for capacity of classical-quantum channels. IEEE Transactions on Information Theory, 49:1753-1768, 2003.

[15] J. Gao. Quantum union bounds for sequential projective measurements. Physical Review A, 92:052331:1-052331:6, 2015.

[16] S. Oskouei, S. Mancini, and M. Wilde. Union bound for quantum information processing. Proceedings of the Royal Society A, 475:20180612:1-20180612:19, 2019.

[17] O. Fawzi, P. Hayden, I. Savov, P. Sen, and M. Wilde. Classical communication over a quantum interference channel. IEEE Transactions on Information Theory, 58:3670-3691, 2012.

[18] S.C. Xu and M. Wilde. Sequential, successive, and simultaneous decoders for entanglement-assisted classical communication. Quantum Information Processing, 12:641-683, 2013.

[19] H. Qi, Q. Wang, and M. Wilde. Applications of positionbased coding to classical communication over quantum channels. Journal of Physics A: Mathematical and Theoretical, 51:444002:1-444002:42, 2018.

[20] N. Dutil. Multiparty quantum protocols for assisted entanglement distillation. $\mathrm{PhD}$ thesis, McGill University, Canada, 2011. Also arXiv:1105.4657.

[21] L. Drescher and O. Fawzi. On simultaneous min-entropy smoothing. In IEEE International Symposium on Information Theory (ISIT), pages 161-165, 2013.

[22] M. Berta, Brandão, F., and C. Hirche. On composite quantum hypothesis testing. Available at arXiv:1709.07268, 2017.

[23] F. Buscemi and N. Datta. The quantum capacity of channels with arbitrarily correlated noise. IEEE Transactions on Information Theory, 56:1447-1460, 2010.

[24] F. Brandão and N. Datta. One-shot rates for entanglement manipulation under non-entangling maps. IEEE Transactions on Information Theory, 57:1754-1760, 2011.

[25] A. Anshu, R. Jain, and N. Warsi. A hypothesis testing approach for communication over entanglement-assisted compound quantum channel. IEEE Transactions on Information Theory, 65:2623-2636, 2019.

[26] A. Harrow, C. Lin, and A. Montanaro. Sequential measurements, disturbance and property testing. In ACM-SIAM Symposium on Discrete Algorithms (SODA), pages 1598-1611, 2017. Full version at arXiv:1607.03236. 
[27] A. Anshu, R. Jain, and N. Warsi. On the near-optimality of one-shot classical communication over quantum channels. Journal of Mathematical Physics, 60:012204:1-012204:19, 2019.

[28] A. Anshu, R. Jain, and N. Warsi. A generalized quantum Slepian-Wolf. IEEE Transactions on Information Theory, 64:1436-1453, 2018.

[29] A. Anshu, R. Jain, and N. Warsi. Building blocks for communication over noisy quantum networks. IEEE Transactions on Information Theory, 65:1287-1306, 2019.

[30] M-H. Hsieh, I. Devetak, and A. Winter. Entanglement-assisted capacity of quantum multiple-access channels. IEEE Transactions on Information Theory, 54:3078-3090, 2008.

\section{Appendix I. Proof of Proposition 4}

We prove Proposition 4 in this section. We first show how to construct the objects whose existence is promised by the proposition. We then proceed to prove its claims. All the while, we use the notation of the proposition: $\mathbf{x}, \mathbf{l}$ will denote computational basis vectors of $\mathcal{X}^{\otimes[c]}, \mathcal{L}^{\otimes([c] \cup[k])}, 0 \leq \delta \leq 1$, and $0 \leq \epsilon_{\mathbf{x},\left(S_{1}, \ldots, S_{l}\right)} \leq 1$.

In Appendix I..1, we show how to construct the perturbed state $\left(\rho^{\prime}\right)_{\mathbf{x}, \delta}^{A_{[k}^{\prime \prime}}$ by tilting the original state $\rho_{\mathbf{x}}^{A_{[k]}}$ appropriately. In Appendix I..2, we construct the 'intersection' projector $\left(\Pi^{\prime}\right)_{\mathbf{x}, \mathbf{l} \delta \delta}^{A_{[k]}^{\prime}}$ by using the technique of matrix-tilted span. In Appendix I..3 we define two complex numbers and a tilting map, and in Appendix I..4 we define two operators required for the statement of Proposition 4 using the combinatorial and algebraic framework of matrix tilting. In Appendix I..5. we prove Claim 1 of Proposition 4 using simple operator inequalities. In Appendix I..6 we prove Claim 2 using the geometry behind the smoothing and augmentation technique described earlier. In Appendix I..7 we prove Claim 3, and in Appendix I..8 we prove Claim 4 using combinatorial properties of the tilting operator. In Appendix I..9 we observe that Claim 5 has already been proven in Appendix I..4 earlier. In Appendix I..10, we prove Claim 6 by invoking the results of Appendix I..2 regarding the geometry of the 'intersection' projector.

Appendix I..1 Constructing $\left(\rho^{\prime}\right)_{\mathbf{x}, \mathbf{l}, \delta}^{A_{[k]}^{\prime \prime}}$

Let $S \subseteq[c] \cup[k], S \cap[k] \neq\{\}$. Define $\bar{S}:=([c] \cup[k]) \backslash S$. Let $\mathbf{l}_{S}$ be a computational basis vector of $\mathcal{L}^{\otimes S}$. We define an isometric embedding $\mathcal{T}_{S, \mathbf{l}_{S}}$ of $\left(\mathcal{H} \otimes \mathbb{C}^{2}\right)^{\otimes(S \cap[k])}$ into $((\mathcal{H} \otimes$ $\left.\left.\mathbb{C}^{2}\right) \otimes \mathcal{L}^{\otimes|S|}\right)^{\otimes(S \cap[k])}$ as follows:

$$
\left(\mathcal{T}_{S, \mathbf{l}_{S}}\left(\mathbf{h}_{S \cap[k]}\right)\right)_{S}:=\left(\mathbf{h}_{S}, \mathbf{l}_{S}\right)
$$

where $s \in S \cap[k], \mathbf{h}_{S \cap[k]}$ is a computational basis vector of $\left(\mathcal{H} \otimes \mathbb{C}^{2}\right)^{\otimes(S \cap[k])}, \mathbf{h}_{s},\left(\mathcal{T}_{S, \mathbf{l}_{S}}\left(\mathbf{h}_{S \cap[k]}\right)\right)_{S}$ are the entries in the $s$ th coordinate of $\mathbf{h}_{S \cap[k]}, \mathcal{T}_{S, \mathbf{l}_{S}}\left(\mathbf{h}_{S \cap[k]}\right)$. Observe that $\mathcal{T}_{S, \mathbf{l}_{S}}$ maps computational basis vectors of $\left(\mathcal{H} \otimes \mathbb{C}^{2}\right)^{\otimes(S \cap[k])}$ to computational basis vectors of $\left(\left(\mathcal{H} \otimes \mathbb{C}^{2}\right) \otimes \mathcal{L}^{\otimes|S|}\right)^{\otimes(S \cap[k])}$. Thus, $\mathcal{T}_{S, \mathbf{l}_{S}}$ is an isometric embedding of $\left(\mathcal{H} \otimes \mathbb{C}^{2}\right)^{\otimes(S \cap[k])}$ into $\left(\left(\mathcal{H} \otimes \mathbb{C}^{2}\right) \otimes\right.$ $\left.\mathcal{L}^{\otimes|S|}\right)^{\otimes(S \cap[k])}$ which further embeds into $A_{S \cap[k]}^{\prime \prime}$ in the natural fashion. Let $\mathbb{1}^{\left(\mathcal{H} \otimes \mathbb{C}^{2}\right)^{\otimes(S \cap[k])}}$ denote the identity embedding of $\left(\mathcal{H} \otimes \mathbb{C}^{2}\right)^{\otimes(S \cap[k])}$ into $A_{(S \cap[k])}^{\prime \prime}$. Observe that the range spaces

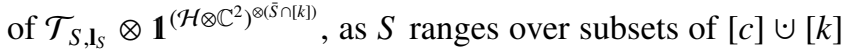
intersecting $[k]$ non-trivially, embed orthogonally into $A_{[k]}^{\prime \prime}$ under the natural embedding. Also, for a fixed $S \subseteq[c] \cup[k]$, $S \cap[k] \neq\{\}$ the range spaces of $\mathcal{T}_{S, \mathbf{l}_{S}}$, as $\mathbf{l}_{S}$ ranges over computational basis vectors of $\mathcal{L}^{\otimes S}$, embed orthogonally into $A_{S \cap[k]}^{\prime \prime}$ under the natural embedding.

We now define an isometric embedding $\mathcal{T}_{S, \mathbf{l}_{S}, \delta}$ of $(\mathcal{H} \otimes$ $\left.\mathbb{C}^{2}\right)^{\otimes(S \cap[k])}$ into $A_{S \cap[k]}^{\prime \prime}$ as follows:

$$
\begin{aligned}
\mathcal{T}_{S, \mathbf{l}_{S}, \delta} & \\
:=\quad \frac{1}{\sqrt{N(S, \delta)}}( & \mathbb{1}^{\left(\mathcal{H} \otimes \mathbb{C}^{2}\right)^{\otimes(S \cap[k])}} \\
& +\sum_{\left(S_{1}, \ldots, S_{l}\right)+\vdash, l>0} \delta^{l} \mathcal{T}_{S_{1}, \mathbf{l}_{S_{1}}} \otimes \cdots \otimes \mathcal{T}_{\left.S_{l}, \mathbf{l}_{S_{l}}\right)} \\
& \left.\otimes \mathbb{1}^{\left(\mathcal{H} \otimes \mathbb{C}^{2}\right)^{\otimes\left((S \cap[k])\left(S_{1} \cup \ldots \cup S_{l}\right)\right)}}\right),
\end{aligned}
$$

where the normalisation factor $N(S, \delta)$ is put in to make the embedding preserve length of vectors. Observe that $N(S, \delta)$ is independent of the vector in $\left(\mathcal{H} \otimes \mathbb{C}^{2}\right)^{\otimes(S \cap[k])}$ on which $\mathcal{T}_{S, \mathbf{l}_{S}, \delta}$ acts since the terms in the above summation have orthogonal range spaces. It can be estimated as follows:

$$
\begin{aligned}
& N(S, \delta):=1+\sum_{\left(S_{1}, \ldots, S_{l}\right) \vdash \vdash, l>0} \delta^{2 l}
\end{aligned}
$$

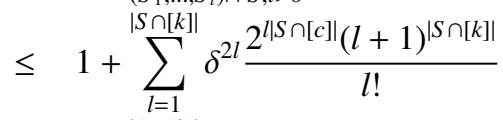

$$
\begin{aligned}
& \leq 1+\sum_{l=1}^{|S \cap[k]|} \frac{\delta^{2 l} 2^{l|S|}}{l !}<e^{\delta^{2} 2^{|S|}} .
\end{aligned}
$$

It is clear that $N(S, \delta) \geq 1$ and $N\left(T_{1}, \delta\right) \cdots N\left(T_{m}, \delta\right) \leq N(S, \delta)$ if $\left(T_{1}, \ldots, T_{m}\right) \vdash \vdash S$.

The map $\mathcal{T}_{S, \mathbf{l}_{S}, \delta}$ extends to subspaces and density matrices in $\left(\mathcal{H} \otimes \mathbb{C}^{2}\right)^{\otimes(S \cap[k])}$ in the natural way. We now define the quantum state

$$
\left(\rho^{\prime}\right)_{\mathbf{x}, \mathbf{l}, \delta}^{A_{[k]}^{\prime \prime}}:=\mathcal{T}_{[c] \cup[k], \mathbf{l}, \delta}\left(\rho_{\mathbf{x}}^{A_{[k]}} \otimes\left(|0\rangle\left\langle\left. 0\right|^{\mathbb{C}^{2}}\right)^{\otimes k}\right)\right.
$$

in $A_{1}^{\prime \prime} \cdots A_{k}^{\prime \prime}$, where $\rho_{\mathbf{x}}^{A_{[k]}} \otimes\left(|0\rangle\left\langle\left. 0\right|^{\mathbb{C}^{2}}\right)^{\otimes k}\right.$ can be thought of as a density matrix in $\left(\mathcal{H} \otimes \mathbb{C}^{2}\right)^{\otimes[k]}$ in the natural fashion.

\section{Appendix I..2 Constructing $\left(\Pi^{\prime}\right)_{\mathbf{x}, \mathbf{l}, \delta}^{A_{[k]}^{\prime \prime}}$}

For each $\mathbf{x},\left(S_{1}, \ldots, S_{l}\right) \vdash \vdash[c] \cup[k], l>0$, let $\Pi_{\mathbf{x},\left(S_{1}, \ldots, S_{l}\right)}^{\prime \prime}$ be the POVM element in $A_{1} \cdots A_{k}$ with the property that

$$
\begin{aligned}
\operatorname{Tr}\left[\left(\Pi^{\prime \prime}\right)_{\mathbf{x},\left(S_{1}, \ldots, S_{l}\right)}^{A_{[k}} \rho_{\mathbf{x}}^{A_{[k]}}\right] & \geq 1-\epsilon_{\mathbf{x},\left(S_{1}, \ldots, S_{l}\right),} \\
\operatorname{Tr}\left[\left(\Pi^{\prime \prime}\right)_{\mathbf{x},\left(S_{1}, \ldots, S_{l}\right)}^{A_{[k]}} \rho_{\mathbf{x},\left(S_{1}, \ldots, S_{l}\right)}^{\left.A_{[k]}\right]}\right. & \leq 2^{-D_{H}^{\epsilon_{\mathbf{x}}\left(S_{1}, \ldots, S_{l}\right)}\left(\rho_{\mathbf{x}}^{A_{[k]}} \| \rho_{\mathbf{x},\left(S_{1}, \ldots, S_{l}\right)}^{A_{[k]}}\right) .}
\end{aligned}
$$

By Fact 2 there exists an orthogonal projection $\Pi_{\mathbf{x},\left(S_{1}, \ldots, S_{l}\right)}$ in $\left(A_{1} \cdots A_{k}\right) \otimes\left(\mathbb{C}^{2}\right)^{\otimes k} \cong\left(\mathcal{H} \otimes \mathbb{C}^{2}\right)^{\otimes[k]}$ such that

$$
\begin{aligned}
& \operatorname{Tr}\left[\Pi_{\mathbf{x},\left(S_{1}, \ldots, S_{l}\right)}^{\left(\mathcal{H} \otimes \mathbb{C}^{2}\right)^{\otimes[k]}}\left(\rho_{\mathbf{x}}^{A_{[k]}} \otimes\left(|0\rangle\left\langle\left. 0\right|^{\mathbb{C}^{2}}\right)^{\otimes k}\right)\right]\right. \\
& \geq 1-\epsilon_{\mathbf{x},\left(S_{1}, \ldots, S_{l}\right)}, \\
& \operatorname{Tr}\left[\Pi_{\mathbf{x},\left(S_{1}, \ldots, S_{l}\right)}^{\left(\mathcal{H} \otimes \mathbb{C}^{2}\right)^{\otimes(k]}}\left(\rho_{\mathbf{x},\left(S_{1}, \ldots, S_{l}\right)}^{A_{[k]}} \otimes\left(|0\rangle\left\langle\left. 0\right|^{\mathbb{C}^{2}}\right)^{\otimes k}\right)\right]\right. \\
& \leq 2^{-D_{H}^{\mathbf{x},\left(S_{1}, \ldots, S_{l}\right)}\left(\rho_{\mathbf{x}}^{A_{k}[k]} \| \rho_{\mathbf{x},\left(S_{1}, \ldots, S_{l}\right)}\right)} .
\end{aligned}
$$


Let $Y_{\mathbf{x},\left(S_{1}, \ldots, S_{l}\right)}$ denote the orthogonal complement of the support of $\Pi_{\mathbf{x},\left(S_{1}, \ldots, S_{l}\right)}$ in $\left(\mathcal{H} \otimes \mathbb{C}^{2}\right)^{\otimes[k]}$. Identify $\left(\mathcal{H} \otimes \mathbb{C}^{2}\right)^{\otimes[k]}$ with the Hilbert space $\mathcal{H}$ of Proposition 3 . Arrange all the non-empty pseudosubpartitions $\left(T_{1}, \ldots, T_{m}\right) \vdash \vdash[c] \cup[k], m>$ 0 into a linear order extending the refinement partial order $\leq$. Define the tilting matrix $A$, whose rows and columns are indexed by non-empty pseudosubpartitions of $[c] \cup[k]$ that intersect $[k]$ non-trivially, as follows:

$$
\begin{aligned}
A_{\left(S_{1}, \ldots, S_{l}\right),\left(T_{1}, \ldots, T_{m}\right)} & \\
:=\quad \frac{\delta^{2 l}}{N\left(T_{1}, \delta\right) \cdots N\left(T_{m}, \delta\right)} & \text { if }\left(S_{1}, \ldots, S_{l}\right) \leq\left(T_{1}, \ldots, T_{m}\right), \\
0 & \text { otherwise. }
\end{aligned}
$$

Observe that $A$ is upper triangular, diagonal dominated and substochastic. The diagonal dominated property of $A$ follows from the fact that $N\left(T_{1}, \delta\right) \cdots N\left(T_{m}, \delta\right) \leq N\left(W_{1}, \delta\right) \cdots N\left(W_{n}, \delta\right)$ if $\left(T_{1}, \ldots, T_{m}\right) \leq\left(W_{1}, \ldots, W_{n}\right)$. The reason $A$ is substochastic in general, and not stochastic, is because the empty pseudosubpartition is not included amongst the rows and columns of $A$. More precisely,

$$
\sum_{\left(S_{1}, \ldots, S_{l}\right):\left(S_{1}, \ldots, S_{l}\right) \leq\left(T_{1}, \ldots, T_{m}\right), l>0} \delta^{2 l}=N\left(T_{1}, \delta\right) \cdots N\left(T_{m}, \delta\right)-1 .
$$

For $\left(S_{1}, \ldots, S_{l}\right) \vdash \vdash[c] \cup[k], l>0$ define an isometric embedding $\mathcal{T}_{\left(S_{1}, \ldots, S_{l}\right), \mathbf{l}, \delta}$ of $\left(\mathcal{H} \otimes \mathbb{C}^{2}\right)^{\otimes[k]}$ into $A_{[k]}^{\prime \prime}$ as follows:

$$
\mathcal{T}_{\left(S_{1}, \ldots, S_{l}\right) \mathbf{l}, \delta}:=\mathcal{T}_{S_{1}, \mathbf{l}_{S_{1}}, \delta} \otimes \cdots \otimes \mathcal{T}_{S_{l}, \mathbf{l}_{S_{l}, \delta}} \otimes \mathbb{1}^{\left(\mathcal{H} \otimes \mathbb{C}^{2}\right)^{\otimes[k] \backslash\left(S_{1} \cup \cdots S_{l}\right)}} .
$$

Observe that the $A$-tilt of $Y_{\mathbf{x},\left(S_{1}, \ldots, S_{l}\right)}$ along the $\left(S_{1}, \ldots, S_{l}\right)$ th direction is nothing but the action of the isometric embedding $\mathcal{T}_{\left(S_{1}, \ldots, S_{l}\right), \mathbf{l}, \delta}:$

$$
\begin{aligned}
& Y_{\mathbf{x},\left(S_{1}, \ldots, S_{l}\right), \mathbf{l}, \delta}^{\prime} \\
& :=\left(\mathcal{T}_{\mathbf{l}}\right)_{\left(S_{1}, \ldots, S_{l}\right), A}\left(Y_{\mathbf{x},\left(S_{1}, \ldots, S_{l}\right)}\right)=\mathcal{T}_{\left(S_{1}, \ldots, S_{l}\right), \mathbf{l}, \delta}\left(Y_{\mathbf{x},\left(S_{1}, \ldots, S_{l}\right)}\right) .
\end{aligned}
$$

Define the $A$-tilted span

$$
Y_{\mathbf{x}, \mathbf{l}, \delta}^{\prime}:=\left(Y_{\mathbf{x}, \mathbf{l}}^{\prime}\right)_{A}=\underset{\left(S_{1}, \ldots, S_{l}\right)+-[c] \cup[k], l>0}{\dagger} Y_{\left(S_{1}, \ldots, S_{l}\right), \mathbf{l}, \delta}^{\prime} .
$$

View $Y_{\mathbf{x}, \mathbf{l}, \delta}^{\prime}$ as a subspace of $A_{1}^{\prime \prime} \ldots A_{k}^{\prime \prime}$. Let $\left(\Pi^{\prime}\right)_{Y_{\mathbf{x}, 1, \delta}^{\prime}}^{A_{[k]}^{\prime \prime}}$ denote the orthogonal projection in $A_{1}^{\prime \prime} \ldots A_{k}^{\prime \prime}$ onto $Y_{\mathbf{x}, \mathbf{l}, \delta}^{\prime}$.

Let $\left(\Pi^{\prime}\right){ }_{\left(\mathcal{H} \otimes \mathbb{C}^{2}\right)^{\otimes[k]}}^{A_{[k]}^{\prime \prime}}$ denote the orthgonal projection in $A_{1}^{\prime \prime} \ldots A_{k}^{\prime \prime}$ onto $\left(\mathcal{H} \otimes \mathbb{C}^{2}\right)^{\otimes[k]}$. We finally define the POVM element $\left(\Pi^{\prime}\right)_{\mathbf{x}, \mathbf{l}, \delta}^{A_{[k]}^{\prime \prime}}$ in $A_{1}^{\prime \prime} \ldots A_{k}^{\prime \prime}$ to be

$$
\left(\Pi^{\prime}\right)_{\mathbf{x}, \mathbf{l}, \delta}^{A_{[k]}^{\prime \prime}}:=\left(\mathbb{1}^{A_{[k]}^{\prime \prime}}-\left(\Pi^{\prime}\right)_{Y_{\mathbf{x}, 1, \delta}^{\prime}}^{A_{[k]}^{\prime \prime}}\right)\left(\left(\Pi^{\prime}\right)_{\left(\mathcal{H} \otimes \mathbb{C}^{2}\right)^{\otimes[k]}}^{A_{1 k}^{\prime \prime}}\right)\left(\mathbb{1}^{A_{[k]}^{\prime \prime}}-\left(\Pi^{\prime}\right)_{Y_{\mathbf{x}, 1, \delta}^{\prime}}^{A_{[k]}^{\prime \prime}}\right)
$$

Appendix I..3 Defining $\alpha_{\left(S_{1}, \ldots, S_{l}\right), \delta}, \beta_{\left(S_{1}, \ldots, S_{l}\right), \delta}, \mathcal{T}_{\left(S_{1}, \ldots, S_{l}\right) \mathbf{1}, \delta}$

Recall that the isometric embedding $\mathcal{T}_{\left(S_{1}, \ldots, S_{l}\right), \mathbf{l}, \delta}$ of $\left(\mathcal{H} \otimes \mathbb{C}^{2}\right)^{\otimes[k]}$ into $A_{[k]}^{\prime \prime}$ has already been defined in Equation A6 above. Also define

$$
\alpha_{\left(S_{1}, \ldots, S_{l}\right), \delta}
$$

$$
\begin{aligned}
:= & \frac{N\left(S_{1}, \delta\right) N\left(\overline{S_{1}} \cup[c], \delta\right)}{N([c] \cup[k], \delta)} \cdots \frac{N\left(S_{l}, \delta\right) N\left(\bar{S}_{l} \cup[c], \delta\right)}{N([c] \cup[k], \delta)}, \\
\alpha_{\left(S_{1}, \ldots, S_{l}\right), \delta}+\beta_{\left(S_{1}, \ldots, S_{l}\right), \delta} & \frac{N\left(S_{1} \cup[c], \delta\right) N\left(\bar{S}_{1} \cup[c], \delta\right)}{N([c] \cup[k], \delta)} \cdots \\
:= & \frac{N\left(S_{l} \cup[c], \delta\right) N\left(\bar{S}_{l} \cup[c], \delta\right)}{N([c] \cup[k], \delta)} .
\end{aligned}
$$

Since

$$
(S, \bar{S} \cup[c]) \leq(S \cup[c], \bar{S} \cup[c]) \vdash \vdash[c] \cup[k]
$$

for any $S \subseteq[c] \cup[k],\{\} \neq S \cap[k] \subset[k]$,

$N(S, \delta) N(\bar{S} \cup[c], \delta) \leq N(S \cup[c], \delta) N(\bar{S} \cup[c], \delta) \leq N([c] \cup[k], \delta)$.

It follows that $0 \leq \alpha_{\left(S_{1}, \ldots, S_{l}\right), \delta}, \beta_{\left(S_{1}, \ldots, S_{l}\right), \delta} \leq 1$.

Appendix I..4 Constructing $M_{\left(S_{1}, \ldots, S_{l}\right), \mathbf{l}, \delta}^{A_{\| k]}^{\prime \prime}}, N_{\left(S_{1}, \ldots, S_{l}\right), \mathbf{l}, \delta}^{A_{[k]}^{\prime \prime}}$

Let $S \subseteq[c] \cup[k],\{\} \neq S \cap[k] \subset[k]$. Define $\bar{S}:=([c] \cup$ $[k]) \backslash S$. For a subset $T \subseteq[c] \cup[k], T \cap[k] \neq\{\}$ we say that $T$ crosses $S$ if $T \cap S \cap[k] \neq\{\}$ and $T \cap \bar{S} \cap[k] \neq$ \{\}. We define a pseudosubpartition $\left(T_{1}, \ldots, T_{l}\right) \vdash \vdash[c] \cup[k]$ to cross $S$ if there exists an $i \in[l]$ such that $T_{i}$ crosses $S$. We use the notation $\left(T_{1}, \ldots, T_{l}\right) \vDash_{\times} S$ to denote that pseudosubpartition $\left(T_{1}, \ldots, T_{l}\right)$ crosses $S$. We define the $S$ signature of a pseudosubpartition $\left(T_{1}, \ldots, T_{l}\right)$ to be the pseudosubpartition $\left(T_{1}, \ldots, T_{l^{\prime}}\right)$ where $l^{\prime} \leq l$ and $T_{1}, \ldots, T_{l^{\prime}}$ are the subsets that actually cross $S$. We shall denote pseudosubpartitions $\left(T_{1}, \ldots, T_{l}\right) \vdash \vdash[c] \cup[k]$ where for all $i \in[l]$, $T_{i}$ crosses $S$, by the notation $\left(T_{1}, \ldots, T_{l}\right) \vDash_{x \times} S$. If pseudosubpartition $\left(T_{1}, \ldots, T_{l}\right)$ has $S$-signature $\left(T_{1}, \ldots, T_{l^{\prime}}\right)$, we shall denote it by $\left(T_{1}, \ldots, T_{l}\right) \wedge_{S}\left(T_{1}, \ldots, T_{l^{\prime}}\right)$. Observe that $\left(T_{1}, \ldots, T_{l^{\prime}}\right) \vDash_{\times x} S$.

From Equation A1, we observe that

$$
\begin{aligned}
& \mathcal{T}_{[c] \cup[k], \mathbf{l}, \delta} \\
& \begin{array}{c}
=\sqrt{\frac{N(S \cup[c], \delta) N(\bar{S} \cup[c], \delta)}{N([c] \cup[k], \delta)}} \mathcal{T}_{S \cup[c], \mathbf{l}_{S} \cup[c], \delta} \otimes \mathcal{T}_{\bar{S} \cup[c], \mathbf{l}_{\bar{S} \cup[c], \delta}} \\
+\frac{1}{\sqrt{N([c] \cup[k], \delta)}} \sum_{\left(T_{1}, \ldots, T_{l}\right) \vdash\left[[c] \cup[k],\left(T_{1}, \ldots, T_{l}\right) \models_{\times} S\right.} \\
\delta^{l}\left(\mathcal{T}_{T_{1}, \mathbf{l}_{T_{1}}} \otimes \cdots \otimes \mathcal{T}_{T_{l}, \mathbf{l}_{T_{l}}} \otimes \mathbb{1}^{\left.\left(\mathcal{H} \otimes \mathbb{C}^{2}\right)^{\left.\otimes(k k]\left(T_{1} \cup \ldots \cup T_{l}\right)\right)}\right) .}\right.
\end{array}
\end{aligned}
$$

We will denote the second term of the summation above by $\mathcal{T}_{\models_{X} S, \mathbf{l}, \delta}$. Observe that $\mathcal{T}_{\models_{\times} S, \mathbf{l}, \delta}$ is a scaled isometric embed$\operatorname{ding}$ of $\left(\mathcal{H} \otimes \mathbb{C}^{2}\right)^{\otimes[k]}$ into $A_{[k]}^{\prime \prime}$.

Let $|h\rangle$ be a unit length vector in $\left(\mathcal{H} \otimes \mathbb{C}^{2}\right)^{\otimes[k]}$. Let the Schmidt decomposition of $|h\rangle$ with respect to $(S \cap[k], \bar{S} \cap[k])$ be

$$
|h\rangle^{\left(\mathcal{H} \otimes \mathbb{C}^{2}\right)^{\otimes[k]}}=\sum_{i} \sqrt{p_{i}}\left|\alpha_{i}\right\rangle^{\left(\mathcal{H} \otimes \mathbb{C}^{2}\right)^{\otimes(S \cap[k])}} \otimes\left|\beta_{i}\right\rangle^{\left(\mathcal{H} \otimes \mathbb{C}^{2}\right)^{\otimes(\bar{S} \cap[k])}},
$$

where $\sum_{i} p_{i}=1$. Let the Schmidt decomposition of $\mathcal{T}_{k_{x} S, \mathbf{l}, \delta}(|h\rangle)$ with respect to $(S \cap[k], \bar{S} \cap[k])$ be

$$
\begin{aligned}
& \left(\mathcal{T}_{\vDash_{\times} S, \mathbf{l}, \delta}(|h\rangle)\right)^{A_{[k]}^{\prime \prime}} \\
& =\sum_{\left(T_{1}, \ldots, T_{l^{\prime}}\right) \models_{\times \times} S} \sum_{j_{\left(T_{1}, \ldots, T_{l^{\prime}}\right)}} \sqrt{q_{j_{\left(T_{1}, \ldots, T_{l^{\prime}}\right)}}}
\end{aligned}
$$

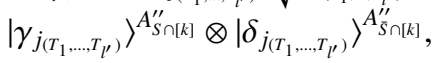


where $\sum_{\left(T_{1}, \ldots, T_{l^{\prime}}\right) \models \times x} \sum_{j_{\left(T_{1}, \ldots, T_{l^{\prime}}\right)}} q_{j_{\left(T_{1}, \ldots, T_{l^{\prime}}\right)}}$ may be less than one reflecting the fact that the length of $\mathcal{T}_{F_{x} S, \mathbf{l}, \delta}(|h\rangle)$ may be less than one. For a pseudosubpartition $\left(T_{1}, \ldots, T_{l^{\prime}}\right) \vDash_{x \times} S$, the expression $\sum_{j_{\left(T_{1}, \ldots, T_{l^{\prime}}\right)}} \sqrt{q_{j_{\left(T_{1}, \ldots, T_{l^{\prime}}\right)}}}\left|\gamma_{j_{\left(T_{1}, \ldots, T_{l^{\prime}}\right)}}\right\rangle^{A_{S \cap[k]}^{\prime \prime}} \otimes\left|\delta_{j_{\left(T_{1}, \ldots, T_{l^{\prime}}\right)}}\right\rangle_{\bar{S} \cap[k]}^{A^{\prime \prime}}$ is the Schmidt decomposition of

$$
\begin{aligned}
& \frac{1}{\sqrt{N([c] \cup[k], \delta)}} \sum_{\left(T_{1}, \ldots, T_{l}\right) \models_{\times} S,\left(T_{1}, \ldots, T_{l}\right) \wedge_{S}\left(T_{1}, \ldots, T_{l^{\prime}}\right)} \\
& \delta^{l}\left(\left(\mathcal{T}_{T_{1}, \mathbf{l}_{T_{1}}} \otimes \cdots \otimes \mathcal{T}_{T_{l}, \mathbf{l}_{T_{l}}} \otimes \mathbb{1}^{\left.\left.\left(\mathcal{H} \otimes \mathbb{C}^{2}\right)^{\otimes\left((k] \backslash\left(T_{1} \cup \cdots \cup T_{l}\right)\right)}\right)(|h\rangle)\right)^{A_{[k]}^{\prime \prime}}}\right.\right.
\end{aligned}
$$

with respect to $(S \cap[k], \bar{S} \cap[k])$. We observe that the span of the vectors $\left\{\left|\gamma_{j_{\left(T_{1}, \ldots, T_{l^{\prime}}\right)}}\right\rangle_{\left.S_{S \cap[k]}^{\prime \prime}\right\}_{j_{\left(T_{1}, \ldots, T_{l^{\prime}}\right.}}}\right.$ is orthogonal to the span of the vectors $\left\{\left|\gamma_{j_{\left(S_{1}, \ldots, S_{m^{\prime}}\right)}}\right\rangle^{\left.A_{S \cap[k]}^{\prime \prime}\right\}_{j_{\left(S_{1}, \ldots, S_{m^{\prime}}\right)}}}\right.$ for different pseudosubpartitions $\left(T_{1}, \ldots, T_{l^{\prime}}\right),\left(S_{1}, \ldots, S_{m^{\prime}}\right) \vDash_{x \times} S$. Similarly, the span of $\left\{\left|\delta_{j_{\left(T_{1}, \ldots, T_{l^{\prime}}\right)}}\right\rangle^{A_{\widetilde{S} \cap[k]}^{\prime \prime}}\right\}_{j_{\left(T_{1}, \ldots, T_{l^{\prime}}\right)}}$ is orthogonal to the span of

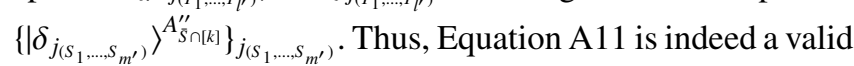
Schmidt decomposition for $\mathcal{T}_{\models \times} S, \mathbf{1}, \delta(|h\rangle)$.

Now,

$$
\begin{aligned}
& \left(\mathcal{T}_{[c] \cup[k], \mathbf{l}, \delta}(|h\rangle)\right)^{A_{[k]}^{\prime \prime}} \\
& =\sqrt{\frac{N(S \cup[c], \delta) N(\bar{S} \cup[c], \delta)}{N([c] \cup[k], \delta)}} \sum_{i} \sqrt{p_{i}} \\
& \left(\mathcal{T}_{S \cup[c], \mathbf{I}_{S \cup[c]}, \delta}\left(\left|\alpha_{i}\right\rangle\right)\right)^{A_{S \cap[k]}^{\prime \prime}} \otimes\left(\mathcal{T}_{\bar{S} \cup[c], \mathbf{l}_{\bar{S} \cup[c], \delta}}\left(\left|\beta_{i}\right\rangle\right)\right)^{A_{\bar{S} \cap[k]}^{\prime \prime}} \\
& +\sum_{\left(T_{1}, \ldots, T_{l^{\prime}}\right) \vdash_{\times \times} S} \sum_{j_{\left(T_{1}, \ldots, T_{l^{\prime}}\right)}} \sqrt{q_{j_{\left(T_{1}, \ldots, T_{l^{\prime}}\right)}}} \\
& \left|\gamma_{j_{\left(T_{1}, \ldots, T_{l^{\prime}}\right)}}\right\rangle^{A_{S \cap[k]}^{\prime \prime}} \otimes\left|\delta_{j_{\left(T_{1}, \ldots, T_{l^{\prime}}\right)}}\right\rangle^{A_{\widetilde{S} \cap[k]}^{\prime \prime}} .
\end{aligned}
$$

where $\sum_{i} s_{i}=1$. Let $\left|\gamma_{j_{\left(T_{1}, \ldots, T_{l^{\prime}}\right)}}(i)\right\rangle, q_{j_{\left(T_{1}, \ldots, T_{l^{\prime}}\right)}}(i)$ be the appropriate vectors and coefficients for $|(h(i))\rangle$ as defined in Equation A11. Then using Equation A3, we get

$$
\begin{aligned}
& \operatorname{Tr}_{A_{\bar{S} \cap[k]}^{\prime \prime}}\left[\left(\rho^{\prime}\right)_{\mathbf{x}, \mathbf{l}, \delta}^{\left.A_{[k]}^{\prime \prime}\right]}\right. \\
& =\frac{N(S \cup[c], \delta) N(\bar{S} \cup[c], \delta)}{N([c] \cup[k], \delta)}
\end{aligned}
$$

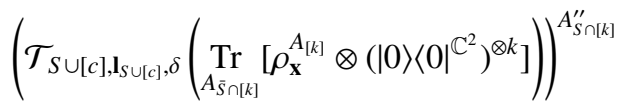

$$
\begin{aligned}
& +\sum_{i} s_{i} \sum_{\left(T_{1}, \ldots, T_{l^{\prime}}\right) \models \times \times} \sum_{j_{\left(T_{1}, \ldots, T_{l^{\prime}}\right)}} q_{j_{\left(T_{1}, \ldots, T_{l^{\prime}}\right)}}(i) \\
& \left|\gamma_{j_{\left(T_{1}, \ldots, T_{l^{\prime}}\right)}}(i)\right\rangle\left\langle\left.\gamma_{j_{\left(T_{1}, \ldots, T_{l^{\prime}}\right)}}(i)\right|^{A_{S \cap[k]}^{\prime \prime}}\right. \\
& =\frac{N(S \cup[c], \delta) N(\bar{S} \cup[c], \delta)}{N([c] \cup[k], \delta)} \\
& \left(\mathcal{T}_{S \cup[c], \mathbf{l}_{S \cup[c]}, \delta}\left(\rho_{\mathbf{x}}^{A_{S \cap[k]}} \otimes\left(|0\rangle\left\langle\left. 0\right|^{\mathbb{C}^{2}}\right)^{\otimes|S \cap[k]|}\right)\right)^{A_{S \cap[k]}^{\prime \prime}}\right. \\
& +\left(M^{\prime \prime}\right)_{S, \mathbf{x}, \mathbf{l}, \delta}^{A_{S \cap k]}^{\prime \prime}}
\end{aligned}
$$

where $\left(M^{\prime \prime}\right)_{S, \mathbf{x}, \mathbf{l}, \delta}^{A_{S}^{\prime \prime}[k]}$ is defined to be the second term in the summation in the first equality above. Note that $\left(M^{\prime \prime}\right)_{S, \mathbf{x}, \mathbf{l}, \delta}^{A_{S \cap[k]}^{\prime \prime}}$ is a positive semidefinite matrix with support orthogonal to the support of the first matrix in the summation above.

We say that a pseudosubpartition $\left(T_{1}, \ldots, T_{l}\right) \vdash \vdash S \cup[c]$ leaks out of $S$ if there exists an $i \in[l]$ such that $T_{i} \cap \bar{S} \cap[c] \neq$ \{\} . We use the notation $\left(T_{1}, \ldots, T_{l}\right) \vDash_{\rightsquigarrow} S$ to denote that pseudosubpartition $\left(T_{1}, \ldots, T_{l}\right)$ leaks out of $S$. Observe that $A_{[k}^{\prime \prime} \frac{\pi}{s}$

Observe that this is a Schmidt decomposition of
that is, for all $i,\left(T_{1}, \ldots, T_{l^{\prime}}\right) \vDash_{\times \times} S, j_{\left(T_{1}, \ldots, T_{l^{\prime}}\right)}$,

$$
\begin{aligned}
& \mathcal{T}_{S \cup[c], \mathbf{l}_{S \cup[c], \delta}}\left(\left|\alpha_{i}\right\rangle\right) \perp\left|\gamma_{\left.j_{\left(T_{1}, \ldots, T_{l^{\prime}}\right)}\right\rangle}\right\rangle \\
& \text { and } \mathcal{T}_{\bar{S} \cup[c], \mathbf{l}_{\bar{S} \cup[c]}, \delta}\left(\left|\beta_{i}\right\rangle\right) \perp\left|\delta_{j_{\left(T_{1}, \ldots, T_{l^{\prime}}\right)}}\right\rangle \text {. } \\
& \text { Thus, }
\end{aligned}
$$

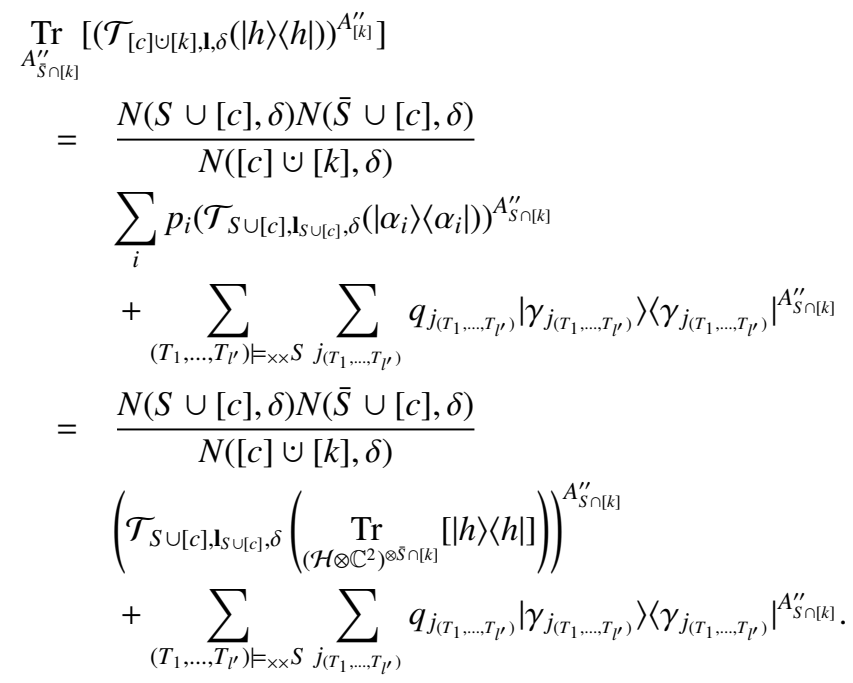

Express $\rho_{\mathbf{x}}^{A_{[k]}} \otimes\left(|0\rangle\left\langle\left. 0\right|^{\mathbb{C}^{2}}\right)^{\otimes k}\right.$ in terms of its eigenbasis

$\rho_{\mathbf{x}}^{A_{[k]}} \otimes\left(|0\rangle\left\langle\left. 0\right|^{\mathbb{C}^{2}}\right)^{\otimes k}=\sum_{i} s_{i}|(h(i))\rangle\left\langle\left.(h(i))\right|^{\left(\mathcal{H} \otimes \mathbb{C}^{2}\right)^{\otimes k}}\right.\right.$,

$$
\begin{aligned}
& =\sqrt{\frac{N(S, \delta)}{N(S \cup[c], \delta)}} \mathcal{T}_{S, \mathbf{l}_{S}, \delta} \\
& +\frac{1}{\sqrt{N(S \cup[c], \delta)}} \sum_{\left(T_{1}, \ldots, T_{l}\right)+\vdash \cup[c],\left(T_{1}, \ldots, T_{l}\right) \models \rightsquigarrow S} \\
& \quad \delta^{l}\left(\mathcal{T}_{T_{1}, \mathbf{l}_{T_{1}}} \otimes \cdots \otimes \mathcal{T}_{T_{l}, \mathbf{l}_{T_{l}}} \otimes \mathbb{1}^{\left.\left(\mathcal{H} \otimes \mathbb{C}^{2}\right)^{\otimes\left((S \cap[k])\left(T_{1} \cup \cdots \cup T_{l}\right)\right)}\right) .}\right.
\end{aligned}
$$

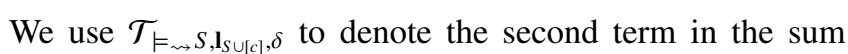
above. The map $\mathcal{T}_{\vDash \rightsquigarrow S, \mathbf{I}_{S \cup[c]}, \delta}$ is a scaled isometric embedding of $\left(\mathcal{H} \otimes \mathbb{C}^{2}\right)^{\otimes(S \cap[k])}$ into $A_{S \cap[k]}^{\prime \prime}$. Let $|h\rangle \in\left(\mathcal{H} \otimes \mathbb{C}^{2}\right)^{\otimes(S \cap[k])}$ be a unit length vector. Define the Hermitian matrix

$$
\begin{aligned}
\left(N_{\mathbf{l}_{S \cup[c]}, \delta}^{\prime \prime}(|h\rangle\langle h|)\right)^{A_{S \cap[k]}^{\prime \prime}} & \quad:=\quad\left(\mathcal{T}_{\left.S \cup[c], \mathbf{l}_{S \cup[c], \delta}(|h\rangle\langle h|)\right)^{A_{S \cap[k]}^{\prime \prime}}}\right. \\
& \quad-\frac{N(S, \delta)}{N(S \cup[c], \delta)}\left(\mathcal{T}_{S, \mathbf{l}_{S}, \delta}(|h\rangle\langle h|)\right)^{A_{S \cap[k]}^{\prime \prime}} .
\end{aligned}
$$

Then,

$$
\begin{aligned}
& \left(N_{\mathbf{l}_{S \cup[c]}, \delta}^{\prime \prime}(|h\rangle\langle h|)\right)^{A_{S \cap[k]}^{\prime \prime}} \\
& =\sqrt{\frac{N(S, \delta)}{N(S \cup[c], \delta)}}\left(\left(\mathcal{T}_{S, \mathbf{l}_{S}, \delta}(|h\rangle) \mathcal{T}_{F_{\rightsquigarrow} S, \mathbf{l}_{S \cup[c]}, \delta}(\langle h|)\right)^{A_{S \cap[k]}^{\prime \prime}}\right. \\
& +\left(\mathcal{T}_{F_{\rightsquigarrow} S, \mathbf{l}_{S \cup[c]}, \delta}(|h\rangle) \mathcal{T}_{S, \mathbf{l}_{S}, \delta}(\langle h|)\right)^{\left.A_{S \cap[k]}^{\prime \prime}\right)} \\
& +\left(\mathcal{T}_{\vDash \rightsquigarrow S, l_{S \cup[c]}, \delta}(|h\rangle\langle h|)\right)^{A_{S \cap[k]}^{\prime \prime}} .
\end{aligned}
$$


Looking at Equation A12, we define the Hermitian matrix

$$
\left(N_{S, \mathbf{x}, \mathbf{l}, \delta}^{\prime \prime}\right)^{A_{S \cap[k]}^{\prime \prime}}:=\sum_{i} s_{i}\left(N_{\mathbf{l}_{S \cup[c]}, \delta}^{\prime \prime}(|h(i)\rangle\langle h(i)|)\right)^{A_{S \cap[k]}^{\prime \prime}} .
$$

Note that $\left(N_{S, \mathbf{x}, \mathbf{l}, \delta}^{\prime \prime}\right)^{A_{S \cap k]}^{\prime \prime}}=0$ if $c=0$. Thus,

$$
\begin{aligned}
\left(\mathcal{T}_{S \cup[c], \mathbf{l}_{S \cup[c]}, \delta}\left(\rho_{\mathbf{x}}^{A_{S \cap[k]}} \otimes\left(|0\rangle\left\langle\left. 0\right|^{\mathbb{C}^{2}}\right)^{\otimes|S \cap[k]|}\right)\right)^{A_{S \cap[k]}^{\prime \prime}}\right. & \\
= & \frac{N(S, \delta)}{N(S \cup[c], \delta)}\left(\mathcal{T}_{S, \mathbf{l}_{S}, \delta}\left(\rho_{\mathbf{x}}^{A_{S \cap[k]}} \otimes\left(|0\rangle\left\langle\left. 0\right|^{\mathbb{C}^{2}}\right)^{\otimes|S \cap[k]|}\right)\right)^{A_{S \cap[k]}^{\prime \prime}}\right. \\
& +\left(N_{S, \mathbf{x}, \mathbf{l}, \delta}^{\prime \prime}\right)^{A_{S \cap[k]}^{\prime \prime}}
\end{aligned}
$$

and

$$
\begin{aligned}
& \operatorname{Tr}_{A_{\bar{S} \cap[k]}^{\prime \prime}}\left[\left(\rho^{\prime}\right)_{\mathbf{x}, \mathbf{l}, \delta}^{\left.A_{[k]}^{\prime \prime}\right]}\right] \\
& =\frac{N(S, \delta) N(\bar{S} \cup[c], \delta)}{N([c] \cup[k], \delta)} \\
& \left(\mathcal{T}_{S, \mathbf{l}_{S}, \delta}\left(\rho_{\mathbf{x}}^{A_{S \cap[k]}} \otimes\left(|0\rangle\left\langle\left. 0\right|^{\mathbb{C}^{2}}\right)^{\otimes|S \cap[k]|}\right)\right)^{A_{S \cap[k]}^{\prime \prime}}\right. \\
& +\frac{N(S \cup[c], \delta) N(\bar{S} \cup[c], \delta)}{N([c] \cup[k], \delta)}\left(N_{S, \mathbf{x}, \mathbf{l}, \delta}^{\prime \prime}\right)^{A_{S \cap[k]}^{\prime \prime}} \\
& +\left(M_{S, \mathbf{x}, \mathbf{l}, \delta}^{\prime \prime}\right)^{A_{S \cap[k]}^{\prime \prime}} \text {. }
\end{aligned}
$$

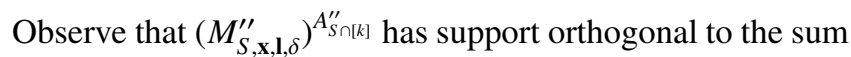
of the first two terms in the above equation and

$$
\operatorname{Tr}\left[\left(M_{S, \mathbf{x}, \mathbf{l}, \delta}^{\prime \prime}\right)^{\left.A_{S \cap[k]}^{\prime \prime}\right]}=1-\frac{N(S \cup[c], \delta) N(\bar{S} \cup[c], \delta)}{N([c] \cup[k], \delta)} .\right.
$$

Also note that the sum of the first two terms is a positive semidefinite matrix.

Now define

$$
\begin{aligned}
& \left(M^{\prime}\right)_{S, \mathbf{x}_{S \cap[c]}, \mathbf{l}_{S}, \delta}^{A_{S}^{\prime \prime}} \\
& :=|\mathcal{L}|^{-|\bar{S}|} \sum_{\mathbf{x}_{[c] \backslash S}^{\prime}, \mathbf{l}_{\bar{S}}^{\prime}} p_{[c] \backslash S}\left(\mathbf{x}_{[c] \backslash S}^{\prime}\right)\left(M^{\prime \prime}\right)_{S, \mathbf{x}_{S \cap[c]} \mathbf{x}_{[c] \backslash S}^{\prime} \mathbf{l}_{S} \mathbf{l}_{\bar{S}}^{\prime}, \delta}^{A_{n}[k]}, \\
& \left(N^{\prime}\right)_{S, \mathbf{x}_{S \cap[c]}, \mathbf{l}_{S}, \delta}^{A_{S \cap[1}^{\prime \prime}} \\
& :=|\mathcal{L}|^{-|\bar{S}|} \frac{N(S \cup[c], \delta) N(\bar{S} \cup[c], \delta)}{N([c] \cup[k], \delta)} \sum_{\mathbf{x}_{[c] \mid S}^{\prime}, \mathbf{l}_{\bar{S}}^{\prime}} p_{[c] \backslash S}\left(\mathbf{x}_{[c] \backslash S}^{\prime}\right) \\
& \left(N^{\prime \prime}\right)_{S, \mathbf{x}_{S \cap[c]} \mathbf{x}_{[c]] S}^{\prime \prime}, \mathbf{l}_{S} \mathbf{l}_{S}^{\prime}, \delta}
\end{aligned}
$$

Obviously, $\left(M^{\prime}\right)_{S, \mathbf{x}_{S \cap[c]}, \mathbf{l}_{S}, \delta}^{A_{S \prime}^{\prime \prime}}$ is a positive semidefinite matrix and $\left(N^{\prime}\right)_{S, \mathbf{x}_{S \cap[c]}, \mathbf{l}_{S}, \delta}^{A_{S}^{\prime \prime}}$ is a Hermitian matrix. Moreover, $\left(N^{\prime}\right)_{S, \mathbf{x}_{S \cap[c]}, \mathbf{l}_{S}, \delta}^{A_{S}^{\prime \prime}}=$ 0 if $c=0$. Recalling the definition of $\left(\rho^{\prime}\right)_{\mathbf{x}_{S \cap[c]}, \mathbf{l}_{S}, \delta}^{A_{S \cap[k}^{\prime \prime}}$ in Claim 5 of Proposition 4, we see that

$$
\begin{aligned}
\left(\rho^{\prime}\right)_{\left.\mathbf{x}_{S \cap[c]}\right] \mathbf{l}_{S}, \delta}^{A_{S \cap[k]}^{\prime \prime}} & \\
= & \frac{N(S, \delta) N(\bar{S} \cup[c], \delta)}{N([c] \cup[k], \delta)} \\
& \left(\mathcal{T}_{S, \mathbf{l}_{S}, \delta}\left(\rho_{\mathbf{x}_{S \cap[c]}}^{A_{S \cap[k]}} \otimes\left(|0\rangle\left\langle\left. 0\right|^{\mathbb{C}^{2}}\right)^{\otimes[S \cap[k] \mid}\right)\right)^{A_{S \cap[k]}^{\prime \prime}}\right. \\
& +\left(N^{\prime}\right)_{S, \mathbf{x}_{S \cap[c]}, \mathbf{l}_{S}, \delta}^{A_{S \cap[k]}^{\prime \prime}}+\left(M^{\prime}\right)_{S, \mathbf{x}_{S \cap[c]}, \mathbf{l}_{S}, \delta}^{A_{S}^{\prime \prime}} .
\end{aligned}
$$

Observe that $\left(M^{\prime}\right)_{S, \mathbf{x}_{S \cap[c]}, \mathbf{l}_{S}, \delta}^{A_{S}^{\prime \prime}}$ has support orthogonal to that of the sum of the first two terms in the above equation and
$\operatorname{Tr}\left[\left(M^{\prime}\right)_{S, \mathbf{x}_{S \cap[c]}, \mathbf{l}_{S}, \delta}^{A_{S}^{\prime \prime}{ }^{\prime}{ }^{\prime}}\right]=1-\frac{N(S \cup[c], \delta) N(\bar{S} \cup[c], \delta)}{N([c] \cup[k], \delta)}$. Also note that the sum of the first two terms is a positive semidefinite matrix.

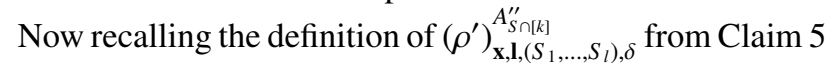
of Proposition 4, we see that

$$
\begin{aligned}
& \left(\rho^{\prime}\right)_{\mathbf{x}, \mathbf{l},\left(S_{1}, \ldots, S_{l}\right), \delta}^{A_{[k]}^{\prime \prime}} \\
& =\left(\frac{N\left(S_{1}, \delta\right) N\left(\overline{S_{1}} \cup[c], \delta\right)}{N([c] \cup[k], \delta)} \cdots \frac{N\left(S_{l}, \delta\right) N\left(\overline{S_{l}} \cup[c], \delta\right)}{N([c] \cup[k], \delta)}\right. \\
& \mathcal{T}_{S_{1}, \mathbf{l}_{S_{1}}, \delta}\left(\rho_{\mathbf{x}_{S_{1} \cap[c]}}^{A_{S_{1} \cap[k]}} \otimes\left(|0\rangle\left\langle\left. 0\right|^{\mathbb{C}^{2}}\right)^{\otimes\left|S_{1} \cap[k]\right|}\right)\right)^{A_{S_{1} \cap[k]}^{\prime \prime} \otimes \cdots \otimes} \\
& \mathcal{T}_{S_{l}, \mathbf{I}_{S_{l}}, \delta}\left(\rho_{\mathbf{x}_{S_{l} \cap[c]}}^{A_{S_{l} \cap[k]}} \otimes\left(|0\rangle\left\langle\left. 0\right|^{\mathbb{C}^{2}}\right)^{\otimes\left|S_{l} \cap[k]\right|}\right)\right)^{A_{S_{l} \cap[k]}^{\prime \prime} \otimes} \\
& \left.\left(\sigma_{\mathbf{x}}^{A_{[k] \backslash\left(S_{1} \cup \cdots \cup s_{l}\right)}} \otimes(|0\rangle\langle 0|)^{\left(\mathbb{C}^{2}\right)^{\otimes[k] \backslash\left(S_{1} \cup \cdots \cup s_{l}\right) \mid}}\right)\right) \\
& + \text { Other Terms I + Other Terms II } \\
& =\quad \alpha_{\left(S_{1}, \ldots, S_{l}\right), \delta}\left(\mathcal{T}_{\left(S_{1}, \ldots, S_{l}\right), \mathbf{l}, \delta}\left(\rho_{\mathbf{x},\left(S_{1}, \ldots, S_{l}\right)}^{A_{[k]}} \otimes\left(|0\rangle\left\langle\left. 0\right|^{\mathbb{C}^{2}}\right)^{\otimes k}\right)\right)^{A_{[k]}^{\prime \prime}}\right.
\end{aligned}
$$

+ Other Terms I + Other Terms II.

Above, the notation "Other Terms II" denotes a $\left(2^{l}-1\right)$-fold sum of tensor products of $(l+1)$ matrices where one mul-

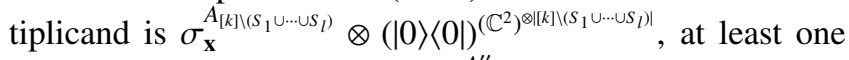
multiplicand is of the form $\left(M^{\prime}\right)_{S_{i}, \mathbf{x}_{S_{i} \cap[c]}, \mathbf{l}_{i}, \delta}^{A_{S_{i}}^{\prime \prime},}$, and the remaining multiplicands are of the form

$$
\begin{aligned}
& \frac{N\left(S_{j}, \delta\right) N\left(\overline{S_{j}} \cup[c], \delta\right)}{N([c] \cup[k], \delta)} \\
& \quad\left(\mathcal{T}_{S_{j}, \mathbf{l}_{S_{j}}, \delta}\left(\rho_{\mathbf{x}_{S_{j} \cap[c]}}^{A_{S_{j} \cap[k]}} \otimes\left(|0\rangle\left\langle\left. 0\right|^{\mathbb{C}^{2}}\right)^{\otimes\left|S_{j} \cap[k]\right|}\right)\right)^{A_{S_{j} \cap[k]}^{\prime \prime}}\right. \\
& \quad+\left(N^{\prime}\right)_{S_{j,}, \mathbf{x}_{S_{j} \cap[c]} A_{S_{j}}^{\prime \prime}, \delta}^{A_{S_{j}}^{\prime \prime}}
\end{aligned}
$$

having $\ell_{1}$-norm at most one. We use $\left(M^{\prime}\right){ }_{\left(S_{1}, \ldots, S_{l}\right), \mathbf{x}, \mathbf{l}, \delta}^{A_{[k]}^{\prime \prime}}$ to denote the "Other Terms II". It is clear that $\left(M^{\prime}\right)_{\left(S_{1}, \ldots, S_{l}\right), \mathbf{x}, \mathbf{l}, \delta}^{A_{(k]}^{\prime \prime}}$ is a positive semidefinite matrix with trace $1-\alpha_{\left(S_{1}, \ldots, S_{l}\right), \delta}-$ $\beta_{\left(S_{1}, \ldots, S_{l}\right), \delta}$. Define

$$
M_{\left(S_{1}, \ldots, S_{l}\right), \mathbf{x}, \mathbf{l}, \delta}^{A_{[k]}^{\prime \prime}}:=\frac{\left(M^{\prime}\right)_{\left(S_{1}, \ldots, S_{l}\right), \mathbf{x}, \mathbf{l}, \delta}^{A_{(\prime)}^{\prime \prime}}}{1-\alpha_{\left(S_{1}, \ldots, S_{l}\right), \delta}-\beta_{\left(S_{1}, \ldots, S_{l}\right), \delta}}
$$

It is now clear that $M_{\left(S_{1}, \ldots, S_{l}\right), \mathbf{x}, \mathbf{l}, \delta}^{A_{[k]}^{\prime \prime}}$ is a positive semidefinite matrix with unit trace with support orthogonal to that of the sum of the first two terms in Equation A16. The notation "Other Terms I" denotes a $\left(2^{l}-1\right)$-fold sum of tensor products of $(l+1)$ matrices where one multiplicand is $\sigma_{\mathbf{x}}^{A_{[k] \backslash\left(S_{1} \cup \cdots \cup S_{l}\right)} \otimes} \otimes$

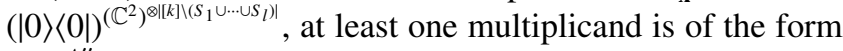
$\left(N^{\prime}\right)_{S_{i}, \mathbf{x}_{S_{i} \cap[c]}, \mathbf{l}_{S_{i}}, \delta}^{A_{1}^{\prime \prime}, \delta^{\prime}}$, and the remaining multiplicands are of the form

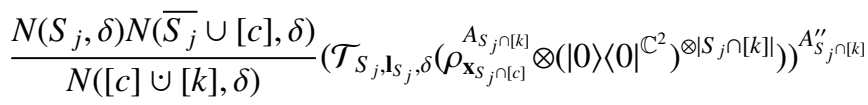
with $\ell_{1}$-norm at most one. We use $\left(N^{\prime}\right)_{\left(S_{1}, \ldots, S_{l}\right), \mathbf{x}, \mathbf{l}, \delta}^{A_{A^{\prime \prime}}^{\prime \prime}}$ to denote the "Other Terms I". It is clear that $\left(N^{\prime}\right)_{\left(S_{1}, \ldots, S_{l}\right), \mathbf{x}, \mathbf{l}, \delta}^{\left.A_{(k]}^{\prime \prime}, \ldots, S_{l}\right), \mathbf{x}, \mathbf{l}, \delta}$ is a Hermitian matrix with trace $\beta_{\left(S_{1}, \ldots, S_{l}\right), \delta}$. Define

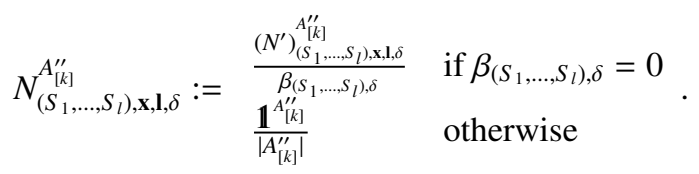


It is now clear that $N_{\left(S_{1}, \ldots, S_{l}\right), \mathbf{x}, \mathbf{l}, \delta}^{A_{[k]}^{\prime \prime}}$ is a Hermitian matrix with unit trace. Also, $\beta_{\left(S_{1}, \ldots, S_{l}\right), \delta}=0$ if $c=0$.

Thus,

$$
\begin{aligned}
\left(\rho^{\prime}\right)_{\mathbf{x}, \mathbf{l},\left(S_{1}, \ldots, S_{l}\right), \delta}^{A_{[k]}^{\prime \prime}} & \\
= & \alpha_{\left(S_{1}, \ldots, S_{l}\right), \delta}\left(\mathcal{T}_{\left(S_{1}, \ldots, S_{l}\right), \mathbf{l}, \delta}\left(\rho_{\mathbf{x},\left(S_{1}, \ldots, S_{l}\right)}^{A_{[k]}} \otimes\left(|0\rangle\left\langle\left. 0\right|^{\mathbb{C}^{2}}\right)^{\otimes k}\right)\right)^{A_{[k]}^{\prime \prime}}\right. \\
& +\beta_{\left(S_{1}, \ldots, S_{l}\right), \delta} N_{\left(S_{1}, \ldots, S_{l}\right), \mathbf{x}, \mathbf{l}, \delta}^{A_{(k]}^{\prime \prime}} \\
& +\left(1-\alpha_{\left(S_{1}, \ldots, S_{l}\right), \delta}-\beta_{\left(S_{1}, \ldots, S_{l}\right), \delta}\right) M_{\left(S_{1}, \ldots, S_{l}\right), \mathbf{x}, \mathbf{l}, \delta}^{A_{(k]}^{\prime \prime}}
\end{aligned}
$$

\section{Appendix I..5 Proving Claim 1}

Using Equation A9, we have

$$
\begin{aligned}
& \left\|\left(\Pi^{\prime}\right)_{\mathbf{x}, \mathbf{l}, \delta}^{A_{[k]}^{\prime \prime}}\right\|_{1} \\
& \leq\left\|\left(\mathbb{1}^{A_{[k]}^{\prime \prime}}-\left(\Pi^{\prime}\right)_{Y_{\mathbf{x}, 1, \delta}^{\prime}}^{A_{[k]}^{\prime \prime}}\right)\right\|_{\infty}\left\|\left(\left(\Pi^{\prime}\right)_{\left(\mathcal{H} \otimes \mathbb{C}^{2}\right)^{\otimes[k]}}^{A^{\prime \prime}}\right)\right\|_{1}
\end{aligned}
$$

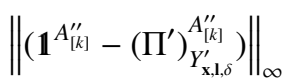

$$
\begin{aligned}
& \leq\left\|\left(\Pi^{\prime}\right)_{\left(\mathcal{H} \otimes \mathbb{C}^{2}\right)^{\otimes[k]}}^{A_{[k]}^{\prime \prime}}\right\|_{1}=\left|\left(\mathcal{H} \otimes \mathbb{C}^{2}\right)^{\otimes k}\right| \\
& =(2|\mathcal{H}|)^{k} \text {, }
\end{aligned}
$$

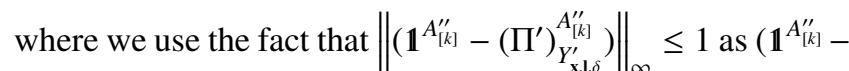
$\left.\left(\Pi^{\prime}\right)_{Y_{\mathbf{x}, 1, \delta}^{\prime}}^{A_{[k]}^{\prime \prime}}\right)$ is the orthogonal projection onto the complement of the subspace $Y_{\mathbf{x}, \mathbf{l}, \delta}^{\prime}$.

\section{Appendix I..6 Proving Claim 2}

Let $S \subseteq[c] \cup[k],\{\} \neq S \cap[k] \neq[k]$. Let $\left|h_{\mathbf{x}}(i)\right\rangle \in\left(\mathcal{H} \otimes \mathbb{C}^{2}\right)^{\otimes k}$ be the $i$ th eigenvector of $\rho_{\mathbf{x}}^{A_{[k]}} \otimes\left(|0\rangle\left\langle\left. 0\right|^{\mathbb{C}^{2}}\right)^{\otimes k}\right.$. Fix a subpartition $\left(T_{1}, \ldots, T_{l^{\prime}}\right) \vDash_{\times x} S$ and an index $j_{\left(T_{1}, \ldots, T_{l^{\prime}}\right)}$ in the Schmidt decomposition of $\mathcal{T}_{F_{x} S, \mathbf{l}, \delta}\left(\left|h_{\mathbf{x}}(i)\right\rangle\right)$ with respect to $(S \cap[k], \bar{S} \cap$ $[k])$ given in Equation A11, Define

$$
\begin{aligned}
& \left(M^{\prime}\right)_{S, \mathbf{x}, \mathbb{I}_{S}, \delta,\left(T_{1}, \ldots, T_{l^{\prime}}\right), j_{\left(T_{1}, \ldots, T_{l^{\prime}}\right)}}^{A_{S \cap}^{\prime \prime}}(i)
\end{aligned}
$$

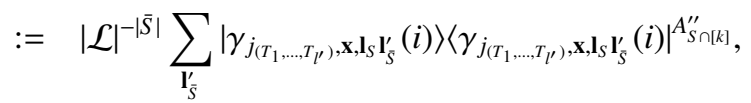

where we write $\left|\gamma_{j_{\left(T_{1}, \ldots, T_{l^{\prime}},\right.}, \mathbf{x}, \mathbf{l}_{S} \mathbf{I}_{\bar{S}}^{\prime}}(i)\right\rangle$ in order to emphasise the dependence on $\mathbf{x}$ and $\mathbf{l}:=\mathbf{l}_{S} \mathbf{l}_{\bar{S}}^{\prime}$.

From the definition of $\left(M^{\prime}\right)_{S, \mathbf{x}_{S \cap[c]}, \mathbf{l}_{S}, \delta}^{A_{S \cap k]}^{\prime \prime}}$ in Equation A15. it is easy to see that proving

$$
\left\|\left(M^{\prime}\right)_{S, \mathbf{x}, \mathbf{l}_{S}, \delta,\left(T_{1}, \ldots, T_{l^{\prime}}\right), j_{\left(T_{1}, \ldots, T_{l^{\prime}}\right)}}^{A_{S \cap k}^{\prime \prime}}(i)\right\|_{\infty} \leq \frac{1}{|\mathcal{L}|}
$$

for all subpartitions $\left(T_{1}, \ldots, T_{l^{\prime}}\right) \vdash_{\times x} S$, indices $j_{\left(T_{1}, \ldots, T_{l^{\prime}}\right)}$ and $i$ suffices to show that

$$
\left\|\left(M^{\prime}\right)_{S, \mathbf{x}_{S \cap[c]}, \mathbf{l}_{S}, \delta}^{A_{S}^{\prime \prime}}\right\|_{\infty} \leq \frac{1}{|\mathcal{L}|} \operatorname{Tr}\left[\left(M^{\prime}\right)_{S, \mathbf{x}_{S \cap[c]}, \mathbf{l}_{S}, \delta}^{A_{S}^{\prime \prime}}\right] .
$$

Now it is easy to see using Equations A16, A17 that this implies that

$$
\left\|\left(M^{\prime}\right)_{\left(S_{1}, \ldots, S_{l}\right), \mathbf{x}, \mathbf{l}, \delta}^{A_{[k]}^{\prime \prime}}\right\|_{\infty} \leq \frac{1}{|\mathcal{L}|} \operatorname{Tr}\left[\left(M^{\prime}\right)_{\left(S_{1}, \ldots, S_{l}\right), \mathbf{x}, \mathbf{l}, \delta}^{A_{[k]}^{\prime \prime}}\right]
$$

$$
\Longrightarrow\left\|M_{\left(S_{1}, \ldots, S_{l}\right), \mathbf{x}, \mathbf{l}, \delta}^{A_{[k]}^{\prime \prime}}\right\|_{\infty} \leq \frac{1}{|\mathcal{L}|} .
$$

It only remains to show $\left\|\left(M^{\prime}\right)_{S, \mathbf{x}, \mathbf{l}_{S}, \delta,\left(T_{1}, \ldots, T_{l^{\prime}}\right), j_{\left(T_{1}, \ldots, T_{l^{\prime}}\right)}}^{A_{S \prime}^{\prime \prime}}(i)\right\|_{\infty} \leq$ $\frac{1}{|\mathcal{L}|}$ for any subpartition $\left(T_{1}, \ldots, T_{l^{\prime}}\right) \vdash_{\times \times} S$, index $j_{\left(T_{1}, \ldots, T_{l^{\prime}}\right)}$ and $i$. In fact, we will prove the stronger statement that

$$
\left\|\left(M^{\prime}\right)_{S, \mathbf{x}, \mathbf{l}_{S}, \delta,\left(T_{1}, \ldots, T_{l^{\prime}}\right), j_{\left(T_{1}, \ldots, T_{l^{\prime}}\right)}}^{A_{\prime \prime \cap[}^{\prime \prime}}(i)\right\|_{\infty} \leq \frac{1}{|\mathcal{L}|^{\left(T_{1} \cup \cdots \cup T_{l^{\prime}}\right) \cap \bar{S} \mid}} .
$$

Since $\left(T_{1} \cup \cdots \cup T_{l^{\prime}}\right) \cap \bar{S} \neq\{\}$, this would complete the proof of the first part of Claim 2 of Proposition 4.

By triangle inequality, we have

$$
\begin{aligned}
& \left\|\left(M^{\prime}\right)_{S, \mathbf{x}, \mathbf{l}_{S}, \delta,\left(T_{1}, \ldots, T_{l^{\prime}}\right), j_{\left(T_{1}, \ldots, T_{l^{\prime}}\right)}}^{A_{S}^{\prime \prime}}(i)\right\|_{\infty} \\
& \leq|\mathcal{L}|^{-\left|\bar{S} \cap\left(T_{1} \cup \cdots T_{l^{\prime}}\right)\right|}|\mathcal{L}|^{-\left|\bar{S} \backslash\left(T_{1} \cup \cdots T_{l^{\prime}}\right)\right|} \sum_{\mathbf{l}_{S \backslash\left(T_{1} \cup \cdots T_{l^{\prime}}\right)}^{\prime}}
\end{aligned}
$$

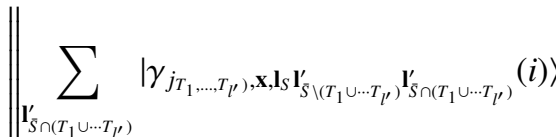

$$
\begin{aligned}
& \left\langle\left.\gamma_{j_{T_{1}, \ldots, T_{l^{\prime}},}, \mathbf{x}, \mathbf{l}_{S} \mathbf{l}_{\bar{S}_{S} \backslash\left(T_{1} \cup \cdots T_{l^{\prime}}\right.}^{\prime} \mathbf{l}_{\bar{S} \cap\left(T_{1} \cup \cdots T_{l^{\prime}}\right)}^{\prime}}(i)\right|^{A_{S \cap[k]}^{\prime \prime}} \|_{\infty}\right. \\
& =|\mathcal{L}|^{-\left|\bar{S} \cap\left(T_{1} \cup \cdots T_{l^{\prime}}\right)\right|}|\mathcal{L}|^{-\left|\bar{S} \backslash\left(T_{1} \cup \cdots T_{l^{\prime}}\right)\right|} \sum_{\mathbf{l}_{S \backslash\left(T_{1} \cup \cdots T_{l^{\prime}}\right)}^{\prime}} 1 \\
& =|\mathcal{L}|^{-\left|\bar{S} \cap\left(T_{1} \cup \cdots T_{l^{\prime}}\right)\right|},
\end{aligned}
$$

where we use the fact that

$$
\begin{aligned}
& \left|\gamma_{\left.j_{\left(T_{1}, \ldots, T_{l}\right)}, \mathbf{x}, \mathbf{l}_{S} \mathbf{l}_{\tilde{S} \backslash\left(S_{1} \cup \ldots \cup S_{l^{\prime}}\right.}\right)^{\prime} \mathbf{I}_{\bar{S} \cap\left(T_{1} \cup \cdots T_{l^{\prime}}\right)}^{\prime}}(i)\right\rangle
\end{aligned}
$$

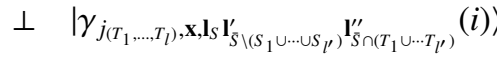

for $\mathbf{l}_{\bar{S} \cap\left(T_{1} \cup \cdots T_{l^{\prime}}\right)}^{\prime} \neq \mathbf{l}_{\bar{S} \cap\left(T_{1} \cup \cdots T_{l^{\prime}}\right)}^{\prime \prime}$ in the equality above. This follows from the observation that for distinct computational basis vectors $\mathbf{l}_{\bar{S} \cap\left(T_{1} \cup \cdots T_{l^{\prime}}\right)}^{\prime}, \mathbf{l}_{\bar{S} \cap\left(T_{1} \cup \cdots T_{l^{\prime}}\right)}^{\prime \prime}$, there exists an $i \in\left[l^{\prime}\right]$, a coordinate $a \in \bar{S} \cap T_{i}$ such that $\mathbf{l}_{a}^{\prime} \neq \mathbf{l}_{a}^{\prime \prime}$ which implies that

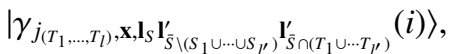

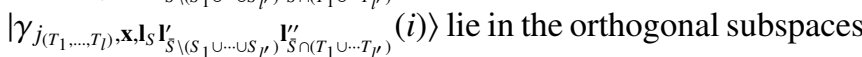
$\left(\left(\mathcal{H} \otimes \mathbb{C}^{2}\right) \otimes\left|\mathbf{l}_{T_{i} \cap S} \mathbf{l}_{T_{i} \cap \bar{S}}^{\prime}\right\rangle\right)_{b} \otimes A_{(S \cap[k]) \backslash\{b\}}^{\prime \prime},\left(\left(\mathcal{H} \otimes \mathbb{C}^{2}\right) \otimes\left|\mathbf{l}_{T_{i} \cap S} \mathbf{l}_{T_{i} \cap \bar{S}}^{\prime \prime}\right\rangle\right)_{b} \otimes$ $A_{(S \cap[k]) \backslash\{b\}}^{\prime \prime}, b \in S \cap[k] \cap T_{i}$, where the first multiplicands in the two tensor products are embedded into $A_{b}^{\prime \prime}$.

This completes the proof of the first part of Claim 2 of Proposition 4

Again, let $S \subseteq[c] \cup[k],\{\} \neq S \cap[k] \neq[k]$. If $[c] \subset S$, then $N_{\mathbf{l}_{S \cup[c], \delta}^{\prime \prime}}^{\prime \prime}(|h\rangle\langle h|)=0$. Suppose $S \cap[c] \neq[c]$. Suppose one were to show that

$$
\left\|\sum_{\mathbf{I}_{[c] S S}^{\prime}}\left(N_{\mathbf{I}_{S} \mathbf{I}_{[c] S S}^{\prime \prime}, \delta}^{\prime}(|h\rangle\langle h|)\right)^{A_{S \cap[k]}^{\prime \prime}}\right\|_{\infty} \leq 3|\mathcal{L}|^{[[c] \backslash S \mid-1 / 2}
$$

for all unit length vectors $|h\rangle \in\left(\mathcal{H} \otimes \mathbb{C}^{2}\right)^{\otimes(S \cap[k])}$. From Equations A14 A15 and the triangle inequality, in either case, it will follow that

$$
\left\|\left(N^{\prime}\right)_{S, \mathbf{x}_{S \cap[c]}, \mathbf{l}_{S}, \delta}^{A_{S}^{\prime \prime}}\right\|_{\infty} \leq \frac{N(S \cup[c], \delta) N(\bar{S} \cup[c], \delta)}{N([c] \cup[k], \delta)} \cdot \frac{3}{\sqrt{|\mathcal{L}|}} .
$$


Now it is easy to see using Equations A16, A18 that this implies that

$$
\beta_{\left(S_{1}, \ldots, S_{l}\right), \delta}\left\|\left(N^{\prime}\right)_{\left(S_{1}, \ldots, S_{l}\right), \mathbf{x}, \mathbf{l}, \delta}^{A_{[k]}^{\prime \prime}}\right\|_{\infty} \leq \frac{3}{\sqrt{|\mathcal{L}|}} .
$$

It only remains to show for $S \cap[c] \neq[c]$ that

$$
\left\|\sum_{I_{[c] \backslash S}^{\prime}}\left(N_{\mathbf{l}_{S} \mathbf{I}_{[c] \backslash S}^{\prime \prime}, \delta}^{\prime \prime}(|h\rangle\langle h|)\right)^{A_{S \cap[k]}^{\prime \prime}}\right\|_{\infty} \leq 3|\mathcal{L}|^{|[c] \backslash S|-1 / 2}
$$

for any unit length vector $|h\rangle \in\left(\mathcal{H} \otimes \mathbb{C}^{2}\right)^{\otimes(S \cap[k])}$. By Equation A13, it suffices to show that

$$
\begin{gathered}
\| \sum_{\mathbf{I}_{[c] \backslash S}^{\prime}} \mathcal{T}_{\models_{\rightsquigarrow} S, \mathbf{l}_{S} \mathbf{I}_{[c] \backslash S}^{\prime}, \delta}(|h\rangle) \|_{2} \leq|\mathcal{L}|^{|[c] \backslash S|-1 / 2}, \\
\left\|\sum_{\mathbf{I}_{[c] \backslash S}^{\prime}} \mathcal{T}_{F_{\rightsquigarrow} S, \mathbf{l}_{S} \mathbf{I}_{[c] S}^{\prime}, \delta}(|h\rangle\langle h|)\right\|_{\infty} \leq|\mathcal{L}|^{|[c] \backslash S|-1} .
\end{gathered}
$$

Since the range spaces of the summands in the definition of $\mathcal{T}_{\vDash \varkappa S, \mathbf{I}_{S \cup[c], \delta}}$ are orthogonal, it suffices to show, for any $\left(T_{1}, \ldots, T_{l}\right)$ เト $S \cup[c],\left(T_{1}, \ldots, T_{l}\right) \vDash_{\rightsquigarrow} S, \mathbf{l}_{S}, \mathbf{l}_{[c] \backslash\left(S \cup T_{1} \cup \ldots \cup T_{l}\right)}^{\prime}$ that

$$
\begin{aligned}
& \| \sum_{\mathbf{l}_{([c] \backslash S) \cap\left(T_{1} \cup \cdots \cup T_{l}\right)}^{\prime \prime}}\left(\mathcal{T}_{T_{1}, \mathbf{l}_{T_{1}}} \otimes \cdots \otimes \mathcal{T}_{T_{l}, \mathbf{l}_{T_{l}}}\right. \\
& \left.\otimes \mathbb{1}^{\left(\mathcal{H} \otimes \mathbb{C}^{2}\right)^{\otimes\left((S \cap[k]) \backslash\left(T_{1} \cup \cdots \cup T_{l}\right)\right)}}\right)(|h\rangle) \|_{2} \\
& =\sqrt{|\mathcal{L}|^{\left|([c] \backslash S) \cap\left(T_{1} \cup \cdots \cup T_{l}\right)\right|}}
\end{aligned}
$$

and

$$
\begin{aligned}
& \| \sum_{\mathbb{l}_{([c] \backslash S) \cap\left(T_{1} \cup \cdots \cup T_{l}\right)}^{\prime \prime}}\left(\mathcal{T}_{T_{1}, \mathbf{l}_{T_{1}}} \otimes \cdots \otimes \mathcal{T}_{T_{l}, \mathbf{l}_{T_{l}}}\right. \\
& \left.\quad \otimes \mathbb{1}^{\left(\mathcal{H} \otimes \mathbb{C}^{2}\right)^{\otimes\left((S \cap[k]) \backslash\left(T_{1} \cup \cdots \cup T_{l}\right)\right)}}\right)(|h\rangle\langle h|) \|_{\infty} \\
& =1 .
\end{aligned}
$$

The last two equalities arise from the fact that for any two distinct computational basis vectors

$$
\mathbf{l}_{([c] \backslash S) \cap\left(T_{1} \cup \cdots \cup T_{l}\right)}^{\prime \prime} \neq \mathbf{l}_{([c] \backslash S) \cap\left(T_{1} \cup \cdots \cup T_{l}\right)}^{\prime \prime \prime}
$$

the range spaces of

$$
\mathcal{T}_{T_{1}, \mathbf{l}_{T_{1}}} \otimes \cdots \otimes \mathcal{T}_{T_{l}, \mathbf{l}_{T_{l}}} \otimes \mathbb{1}^{\left(\mathcal{H} \otimes \mathbb{C}^{2}\right)^{\otimes\left((S \cap[k]) \backslash\left(T_{1} \cup \cdots \cup T_{l}\right)\right)}}
$$

are orthogonal. This follows from the observation that there exists an $i \in[l]$, a coordinate $a \in[c] \cap \bar{S} \cap T_{i}$ such that $\mathbf{l}_{a}^{\prime \prime} \neq$ $\mathbf{l}_{a}^{\prime \prime \prime}$ which implies that the two range spaces embed into the orthogonal spaces $\left(\left(\mathcal{H} \otimes \mathbb{C}^{2}\right) \otimes\left|\mathbf{l}_{T_{i} \cap S} \mathbf{l}_{T_{i} \cap \bar{S} \cap[c]}^{\prime \prime}\right\rangle\right)_{b} \otimes A_{(S \cap[k]) \backslash\{b\}}^{\prime \prime}$, $\left(\left(\mathcal{H} \otimes \mathbb{C}^{2}\right) \otimes\left|\mathbf{l}_{T_{i} \cap S} \mathbf{l}_{T_{i} \cap \bar{S} \cap[c]}^{\prime \prime}\right\rangle\right)_{b} \otimes A_{(S \cap[k]) \backslash\{b\}}^{\prime \prime}, b \in S \cap[k] \cap T_{i}$, where the first multiplicands in the two tensor products are embedded into $A_{b}^{\prime \prime}$.

This completes the proof of the second part of Claim 2 of Proposition 4

\section{Appendix I..7 Proving Claim 3}

Let $|h\rangle$ be a unit length vector in $\left(\mathcal{H} \otimes \mathbb{C}^{2}\right)^{\otimes k}$. From Equation A1 and Inequality A2, we get

$$
\left\langle\mathcal{T}_{[c] \cup[k], \mathbf{l}, \delta}(|h\rangle) \mid h\right\rangle=\frac{1}{\sqrt{N([c] \cup[k], \delta)}}>e^{-\delta^{2} 2^{c+k-1}} .
$$

Thus,

$$
\begin{aligned}
& \| \mathcal{T}_{[c] \cup[k], \mathbf{l}, \delta}(|h\rangle\langle h|)-|h\rangle\langle h| \|_{1} \\
& \quad \leq \quad 2 \| \mathcal{T}_{[c] \cup[k], \mathbf{l}, \delta}(|h\rangle)-|h\rangle \|_{2} \\
& \quad \leq 2 \sqrt{2-2 e^{-\delta^{2} 2^{c+k-1}}}<2^{\frac{c+k}{2}+1} \delta,
\end{aligned}
$$

where we used the fact that $e^{-x} \geq 1-x$ in the last inequality above. Recalling Equation A3 and applying the above inequality to the eigenvectors of $\rho^{A_{[k]}} \otimes\left(|0\rangle\left\langle\left. 0\right|^{\mathbb{C}^{2}}\right)^{\otimes k}\right.$ allows us to prove the desired Claim 3 of Proposition 4.

\section{Appendix I..8 Proving Claim 4}

Let $|h\rangle$ be an eigenvector of $\rho^{A_{[k]}} \otimes\left(|0\rangle\left\langle\left. 0\right|^{\mathbb{C}^{2}}\right)^{\otimes k}\right.$. For a pseudosubpartition $\left(T_{1}, \ldots, T_{m}\right) \vdash \vdash[c] \cup[k]$, define

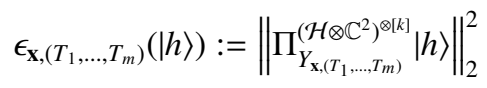

where the subspace $Y_{\mathbf{x},\left(T_{1}, \ldots, T_{m}\right)} \leq\left(\mathcal{H} \otimes \mathbb{C}^{2}\right)^{\otimes[k]}$ is defined just after Equation A4 above. Recall that the subspace $Y_{\mathbf{x}, \mathbf{l}, \delta}^{\prime}$ defined in Equation $\bar{A} 8$ is the $A$-tilted span of $\left\{Y_{\mathbf{x},\left(T_{1}, \ldots, T_{m}\right)}\right.$ : $\left.\left(T_{1}, \ldots, T_{m}\right) \vdash \vdash[c] \cup[k], m>0\right\}$ where the tilting matrix $A$ is defined in Equation A5 above. Recall that $A$ is upper triangular, substochastic and diagonal dominated, and the diagonal entries of $A$ satisfy

$$
\begin{aligned}
& A_{\left(T_{1}, \ldots, T_{m}\right),\left(T_{1}, \ldots, T_{m}\right)} \\
& \quad=\frac{\delta^{2 m}}{N\left(T_{1}, \delta\right) \cdots N\left(T_{m}, \delta\right)} \\
& \quad \geq \frac{\delta^{2 k}}{N([c] \cup[k], \delta)} \geq e^{-\delta^{2} 2^{c+k}} \delta^{2 k},
\end{aligned}
$$

for all $\left(T_{1}, \ldots, T_{m}\right) \vdash \vdash[c] \cup[k]$. By Proposition 3 .

$$
\begin{aligned}
& \|\left(\Pi^{\prime}\right)_{Y_{\mathbf{x}, 1, \delta}^{\prime}}^{A_{[k]}^{\prime \prime}}|h\rangle \|_{2}^{2} \\
& \quad \leq e^{\delta^{2} 2^{c+k}} \delta^{-2 k} 2^{2^{c k+1}(k+1)^{k}+1} \sum_{\left(T_{1}, \ldots, T_{m}\right) \vdash[[c] \cup[k], m>0} \epsilon_{\mathbf{x},\left(T_{1}, \ldots, T_{m}\right)}(|h\rangle) .
\end{aligned}
$$

Using Equation A4 and applying this inequality to the eigenvectors of $\rho^{A_{[k]}} \otimes\left(|0\rangle\left\langle\left. 0\right|^{\mathbb{C}^{2}}\right)^{\otimes k}\right.$, we get

$$
\begin{aligned}
\operatorname{Tr} & {\left[\left(\Pi^{\prime}\right)_{Y_{\mathbf{x}, 1, \delta}^{\prime}}^{A_{[k]}^{\prime \prime}}\left(\rho^{A_{[k]}} \otimes\left(|0\rangle\left\langle\left. 0\right|^{\mathbb{C}^{2}}\right)^{\otimes k}\right)\right]\right.} \\
\leq & e^{\delta^{2} 2^{c+k}} \delta^{-2 k} 2^{2^{c k+1}(k+1)^{k}+1} \sum_{\left(T_{1}, \ldots, T_{m}\right)+\vdash[c] \cup[k], m>0} \epsilon_{\mathbf{x},\left(T_{1}, \ldots, T_{m}\right)} \\
& <\delta^{-2 k} 2^{2^{c k+3}(k+1)^{k}} \epsilon_{\mathbf{x}} .
\end{aligned}
$$

Finally, using Fact 3 and Equation A9, we get

$$
\operatorname{Tr}\left[\left(\Pi^{\prime}\right)_{\mathbf{x}, \mathbf{l}, \delta}^{A_{[k]}^{\prime \prime}}\left(\rho^{A_{[k]}} \otimes\left(|0\rangle\left\langle\left. 0\right|^{\mathbb{C}^{2}}\right)^{\otimes k}\right)\right]\right.
$$




$$
\begin{aligned}
& =\operatorname{Tr}\left[\left(\Pi^{\prime}\right)_{\left(\mathcal{H} \otimes \mathbb{C}^{2}\right)^{\otimes[k]}}^{A_{[k]}^{\prime \prime}}\left(\mathbb{1}_{[k]}^{A_{[k]}^{\prime \prime}}-\left(\Pi^{\prime}\right)_{Y_{\mathbf{x}, 1, \delta}^{\prime}}^{A_{[k]}^{\prime \prime}}\right)\right. \\
& \left(\rho^{A_{[k]}} \otimes\left(|0\rangle\left\langle\left. 0\right|^{\mathbb{C}^{2}}\right)^{\otimes k}\right)\left(\mathbb{1}^{A_{[k]}^{\prime \prime}}-\left(\Pi^{\prime}\right)_{Y_{\mathbf{x}, 1, \delta}^{\prime}}^{A_{k k]}^{\prime \prime}}\right)\right. \\
& \left(\Pi^{\prime}\right)_{\left(\mathcal{H} \otimes \mathbb{C}^{2}\right)^{\otimes[k]}}^{\left.A_{[k]}^{\prime \prime}\right]} \\
& \geq 1-4\left(\operatorname { T r } \left[\left(\Pi^{\prime}\right)_{Y_{\mathbf{x}, 1, \delta}^{\prime}}^{A_{[k]}^{\prime \prime}}\left(\rho^{A_{[k]}} \otimes\left(|0\rangle\left\langle\left. 0\right|^{\mathbb{C}^{2}}\right)^{\otimes k}\right)\right]\right.\right. \\
& +1-\operatorname{Tr}\left[\left(\Pi^{\prime}\right)_{\left(\mathcal{H} \otimes \mathbb{C}^{2}\right)^{\otimes[k]}}^{A_{[k]}^{\prime \prime}}\left(\rho^{A_{[k]}} \otimes\left(|0\rangle\left\langle\left. 0\right|^{\mathbb{C}^{2}}\right)^{\otimes k}\right)\right]\right) \\
& >1-4\left(\delta^{-2 k} 2^{2^{c k+3}(k+1)^{k}} \epsilon_{\mathbf{x}}\right) \geq 1-\delta^{-2 k} 2^{2^{c k+4}(k+1)^{k}} \epsilon_{\mathbf{x}} \text {. }
\end{aligned}
$$

\section{Appendix I..9 Proving Claim 5}

This claim and the accompanying claims of orthogonality and vanishing of $\beta_{\left(S_{1}, \ldots, S_{l}\right), \delta}$ are proved at the end of Appendix I..4 above.

\section{Appendix I..10 Proving Claim 6}

Using Equations A9, A8, A7, A4, we get

$$
\begin{aligned}
& \operatorname{Tr}\left[\left(\Pi^{\prime}\right)_{\mathbf{x}, \mathbf{l}, \delta}^{A_{[k]}^{\prime \prime}}\left(\mathcal{T}_{\left(S_{1}, \ldots S_{l}\right), \mathbf{l}, \delta}\left(\rho_{\mathbf{x},\left(S_{1}, \ldots, S_{l}\right)}^{A_{[k]}} \otimes\left(|0\rangle\left\langle\left. 0\right|^{\mathbb{C}^{2}}\right)^{\otimes k}\right)\right)^{\left.A_{[k]}^{\prime \prime}\right]}\right]\right. \\
& =\operatorname{Tr}\left[\left(\Pi^{\prime}\right)_{\left(\mathcal{H} \otimes \mathbb{C}^{2}\right)^{\otimes k}}^{A_{1 k}^{\prime \prime}}\left(\mathbb{1}^{A_{[k]}^{\prime \prime}}-\left(\Pi^{\prime}\right)_{Y_{\mathbf{x}, 1, \delta}^{\prime}}^{A^{\prime \prime}}\right)\right. \\
& \left(\mathcal{T}_{\left(S_{1}, \ldots S_{l}\right), \mathbf{l}, \delta}\left(\rho_{\mathbf{x},\left(S_{1}, \ldots, S_{l}\right)}^{A_{[k]}} \otimes\left(|0\rangle\left\langle\left. 0\right|^{\mathbb{C}^{2}}\right)^{\otimes k}\right)\right)^{A_{[k]}^{\prime \prime}}\right. \\
& \left(\mathbb{1}^{A_{[k]}^{\prime \prime}}-\left(\Pi^{\prime}\right)_{Y_{\mathbf{x}, 1, \delta}^{\prime}}^{A_{[k]}^{\prime \prime}}\right)\left(\Pi^{\prime}\right)_{\left(\mathcal{H} \otimes \mathbb{C}^{2}\right)^{\otimes k}}^{\left.A_{[k]}^{\prime \prime}\right]} \\
& \leq \operatorname{Tr}\left[\left(\mathbb{1}^{A_{[k]}^{\prime \prime}}-\left(\Pi^{\prime}\right)_{Y_{\mathbf{x}, 1, \delta}^{\prime}}^{A_{[k]}^{\prime \prime}}\right)\right. \\
& \left(\mathcal{T}_{\left(S_{1}, \ldots S_{l}\right), \mathbf{l}, \delta}\left(\rho_{\mathbf{x},\left(S_{1}, \ldots, S_{l}\right)}^{A_{[k]}} \otimes\left(|0\rangle\left\langle\left. 0\right|^{\mathbb{C}^{2}}\right)^{\otimes k}\right)\right)^{A_{[k]}^{\prime \prime}}\right. \\
& \left.\left(\mathbb{1}^{A_{[k]}^{\prime \prime}}-\left(\Pi^{\prime}\right)_{Y_{\mathbf{x}, 1, \delta}^{\prime}}^{A_{[k]}^{\prime \prime}}\right)\right] \\
& \leq \operatorname{Tr}\left[\left(\mathbf{1}_{[k]}^{A_{[k]}^{\prime \prime}}-\Pi_{\mathcal{T}_{\left(S_{1}, \ldots, S_{l}\right), \mathbf{l}, \delta}\left(Y_{\mathbf{x},\left(S_{1}, \ldots, S_{l}\right)}\right)}^{A_{[k]}^{\prime \prime}}\right)\right. \\
& \left(\mathcal{T}_{\left(S_{1}, \ldots S_{l}\right), \mathbf{l}, \delta}\left(\rho_{\mathbf{x},\left(S_{1}, \ldots, S_{l}\right)}^{A_{[k]}} \otimes\left(|0\rangle\left\langle\left. 0\right|^{\mathbb{C}^{2}}\right)^{\otimes k}\right)\right)^{A_{[k]}^{\prime \prime}}\right. \\
& \left.\left(\mathbb{1}^{A_{[k]}^{\prime \prime}}-\Pi_{\mathcal{T}_{\left(S_{1}, \ldots, S_{l}\right), 1, \delta}\left(Y_{\mathbf{x},\left(S_{1}, \ldots, \cup S_{l}\right)}\right)}^{A_{[k]}^{\prime \prime}}\right)\right]
\end{aligned}
$$

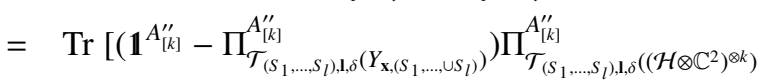

$$
\begin{aligned}
& \left(\mathcal{T}_{\left(S_{1}, \ldots S_{l}\right), \mathbf{l}, \delta}\left(\rho_{\mathbf{x},\left(S_{1}, \ldots, S_{l}\right)}^{A_{[k]}} \otimes\left(|0\rangle\left\langle\left. 0\right|^{\mathbb{C}^{2}}\right)^{\otimes k}\right)\right)^{A_{[k]}^{\prime \prime}}\right. \\
& \left.\Pi_{\mathcal{T}_{\left(S_{1}, \ldots, S_{l}\right), 1, \delta}\left(\left(\mathcal{H} \otimes \mathbb{C}^{2}\right)^{\otimes k}\right)}^{A_{[k]}^{\prime \prime}}\left(\mathbb{1}^{A_{[k]}^{\prime \prime}}-\prod_{\mathcal{T}_{\left(S_{1}, \ldots, S_{l}\right), 1, \delta}\left(Y_{\mathbf{x},\left(S_{1}, \ldots, S_{l}\right)}\right)}^{A_{[k]}^{\prime \prime}}\right)\right] \\
& =\operatorname{Tr}\left[\left(\Pi_{\mathcal{T}_{\left(S_{1}, \ldots, S_{l}\right), 1, \delta}\left(\left(\mathcal{H} \otimes \mathbb{C}^{2}\right)^{\otimes k}\right)}^{A_{[k]}^{\prime \prime}}-\Pi_{\mathcal{T}_{\left(S_{1}, \ldots, S_{l}\right), 1, \delta}\left(Y_{\mathbf{x},\left(S_{1}, \ldots, \cup S_{l}\right)}\right)}^{A_{k k]}^{\prime \prime}}\right)\right. \\
& \left(\mathcal{T}_{\left(S_{1}, \ldots S_{l}\right), \mathbf{l}, \delta}\left(\rho_{\mathbf{x},\left(S_{1}, \ldots, S_{l}\right)}^{A_{[k]}} \otimes\left(|0\rangle\left\langle\left. 0\right|^{\mathbb{C}^{2}}\right)^{\otimes k}\right)\right)\right. \\
& \left.\left(\Pi_{\mathcal{T}_{\left(S_{1}, \ldots, \ldots, l\right), 1, \delta}\left(\left(\mathcal{H} \otimes \mathbb{C}^{2}\right)^{\otimes k}\right)}^{A_{[k]}^{\prime \prime}}-\Pi_{\mathcal{T}_{\left(S_{1}, \ldots, S_{l}\right), 1, \delta}\left(Y_{\mathbf{x},\left(S_{1}, \ldots, S_{l}\right)}\right)}^{A_{[k]}^{\prime \prime}}\right)\right]
\end{aligned}
$$

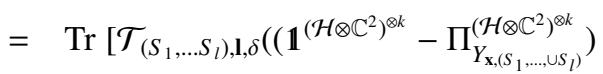

$$
\begin{aligned}
& \left(\rho_{\mathbf{x},\left(S_{1}, \ldots, S_{l}\right)}^{A_{[k]}} \otimes\left(|0\rangle\left\langle\left. 0\right|^{\mathbb{C}^{2}}\right)^{\otimes k}\right)\right.
\end{aligned}
$$

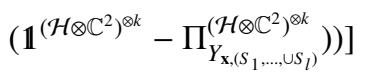

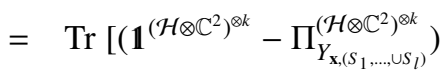

$$
\begin{aligned}
& \left(\rho_{\mathbf{x},\left(S_{1}, \ldots, S_{l}\right)}^{A_{[k]}} \otimes\left(|0\rangle\left\langle\left. 0\right|^{\mathbb{C}^{2}}\right)^{\otimes k}\right)\right.
\end{aligned}
$$

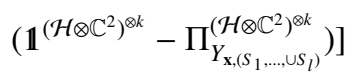

$$
\begin{aligned}
& =\operatorname{Tr}\left[\Pi_{\mathbf{x},\left(S_{1}, \ldots, S_{l}\right)}^{\left(\mathcal{H} \otimes \mathbb{C}^{2} \otimes k\right.}\left(\rho_{\mathbf{x},\left(S_{1}, \ldots, S_{l}\right)}^{A_{[k]}} \otimes\left(|0\rangle\left\langle\left. 0\right|^{\mathbb{C}^{2}}\right)^{\otimes k}\right) \Pi_{\mathbf{x},\left(S_{1}, \ldots, S_{l}\right)}^{\left(\mathcal{H} \otimes \mathbb{C}^{2}\right)^{\otimes k}}\right]\right. \\
& \leq 2^{\left.-D_{H}^{\epsilon_{\mathbf{x},\left(S_{1}, \ldots, S_{l}\right)}^{A_{[}}\left(\rho_{\mathbf{x}} \|\right]} \| \rho_{\mathbf{x},\left(S_{1}, \ldots, S_{l}\right)}^{A_{[k]}}\right)}
\end{aligned}
$$

In the second inequality above, we used the fact that

$$
\mathcal{T}_{\left(S_{1}, \ldots, S_{l}\right), \mathbf{l}, \delta}\left(Y_{\mathbf{x},\left(S_{1}, \ldots, \cup S_{l}\right)}\right) \leq Y_{\mathbf{x}, \mathbf{l}, \delta}^{\prime} .
$$

In the second equality above, we used the property that the support of $\mathcal{T}_{\left.S_{1}, \ldots S_{l}\right), \mathbf{1}, \delta}\left(\rho_{\mathbf{x},\left(S_{1}, \ldots, S_{l}\right)}^{A_{[k]}} \otimes\left(|0\rangle\left\langle\left. 0\right|^{\mathbb{C}^{2}}\right)^{\otimes k}\right)\right.$ lies in the vector space $\mathcal{T}_{\left(S_{1}, \ldots, S_{l}\right), \mathbf{l}, \delta}\left(\left(\mathcal{H} \otimes \mathbb{C}^{2}\right)^{\otimes k}\right)$. We used the property that the map $\mathcal{T}_{\left(S_{1}, \ldots S_{l}\right), \mathbf{l}, \delta}$ is an isometric embedding of $\left.\left(\mathcal{H} \otimes \mathbb{C}^{2}\right)^{\otimes k}\right)$ in the fourth and fifth equalities. Finally, we used the definition of $Y_{\mathbf{x},\left(S_{1}, \ldots, \cup S_{l}\right)}$ given just below Equation $\mathrm{A} 4$ for obtaining the last equality.

This completes the proof of Claim 6 of Proposition 4 and thus finishes the proof of Proposition 4

\section{Appendix II. Proof of Equation 2}

The probability of incorrectly decoding the sent message pair $\left(m_{1}, m_{2}\right)$, for a given codebook $C$, is upper bounded by

$$
\begin{aligned}
p_{e}(C ; & \left.m_{1}, m_{2}\right) \\
\leq & \sum_{z} p\left(z \mid x\left(m_{1}\right), y\left(m_{2}\right)\right) \\
& \sum_{\left(\hat{m}_{1}, \hat{m}_{2}\right):\left(\hat{m}_{1}, \hat{m}_{2}\right)<\left(m_{1}, m_{2}\right)} f\left(x\left(\hat{m}_{1}\right), y\left(\hat{m}_{2}\right), z\right) \\
& +\sum_{z} p\left(z \mid x\left(m_{1}\right), y\left(m_{2}\right)\right)\left(1-f\left(x\left(m_{1}\right), y\left(m_{2}\right), z\right)\right) \\
\leq & \sum_{z} p\left(z \mid x\left(m_{1}\right), y\left(m_{2}\right)\right) \\
& +\sum_{z} p\left(z \mid x\left(m_{1}\right), y\left(m_{2}\right)\right)\left(1-f\left(x\left(m_{1}\right), y\left(m_{2}\right), z\right)\right) \\
= & \sum_{z} p\left(z \mid x\left(m_{1}\right), y\left(m_{2}\right)\right) \sum_{\left(\hat{m}_{1}, \hat{m}_{2}\right): \hat{m}_{1} \neq m_{1}, \hat{m}_{2} \neq m_{2}} f\left(x\left(\hat{m}_{1}\right), y\left(\hat{m}_{2}\right), z\right) \\
& +\sum_{z} p\left(z \mid x\left(m_{1}\right), y\left(m_{2}\right)\right) \sum_{\hat{m}_{2}: \hat{m}_{2} \neq m_{2}} f\left(x\left(m_{1}\right), y\left(\hat{m}_{2}\right), z\right) \\
& +\sum_{z} p\left(z \mid x\left(m_{1}\right), y\left(m_{2}\right)\right) \sum_{\hat{m}_{1}: \hat{m}_{1} \neq m_{1}} f\left(x\left(\hat{m}_{1}\right), y\left(m_{2}\right), z\right) \\
& +\sum_{z} p\left(z \mid x\left(m_{1}\right), y\left(m_{2}\right)\right)\left(1-f\left(x\left(m_{1}\right), y\left(m_{2}\right), z\right)\right) .
\end{aligned}
$$

The expectation, over the choice of the random codebook $C$, of the decoding error is then upper bounded by

$$
\begin{aligned}
\underset{C}{\mathbf{E}}\left[p_{e}\left(C ; m_{1}, m_{2}\right)\right] \\
\leq \quad\left(2^{R_{1}}-1\right)\left(2^{R_{2}}-1\right) \\
\quad \sum_{x, y, z} p(x) p(y) p(z \mid x, y) \sum_{x^{\prime}, y^{\prime}} p\left(x^{\prime}\right) p\left(y^{\prime}\right) f\left(x^{\prime}, y^{\prime}, z\right) \\
\quad+\left(2^{R_{2}}-1\right) \sum_{x, y, z} p(x) p(y) p(z \mid x, y) \sum_{y^{\prime}} p\left(y^{\prime}\right) f\left(x, y^{\prime}, z\right)
\end{aligned}
$$




$$
\begin{aligned}
& +\left(2^{R_{1}}-1\right) \sum_{x, y, z} p(x) p(y) p(z \mid x, y) \sum_{x^{\prime}} p\left(x^{\prime}\right) f\left(x^{\prime}, y, z\right) \\
& +\sum_{x, y, z} p(x) p(y) p(z \mid x, y)(1-f(x, y, z)) \\
& \sum_{x}^{a} \quad\left(2^{R_{1}}-1\right)\left(2^{R_{2}}-1\right) \\
& \quad \sum_{x, y, z} p(x) p(y) p(z \mid x, y) \sum_{x^{\prime}, y^{\prime}} p\left(x^{\prime}\right) p\left(y^{\prime}\right) f^{X, Y}\left(x^{\prime}, y^{\prime}, z\right) \\
& +\left(2^{R_{2}}-1\right) \sum_{x, y, z} p(x) p(y) p(z \mid x, y) \sum_{y^{\prime}} p\left(y^{\prime}\right) f^{Y}\left(x, y^{\prime}, z\right) \\
& +\left(2^{R_{1}}-1\right) \sum_{x, y, z} p(x) p(y) p(z \mid x, y) \sum_{x^{\prime}} p\left(x^{\prime}\right) f^{X}\left(x^{\prime}, y, z\right) \\
& +\sum_{x, y, z} p(x) p(y) p(z \mid x, y)\left(\left(1-f^{X}(x, y, z)\right)\right. \\
& \left.\quad+\left(1-f^{Y}(x, y, z)\right)+\left(1-f^{X, Y}(x, y, z)\right)\right) \\
& \left(2^{R_{1}}-1\right)\left(2^{R_{2}}-1\right) \sum_{x^{\prime}, y^{\prime}, z} p\left(x^{\prime}\right) p\left(y^{\prime}\right) p(z) f^{X, Y}\left(x^{\prime}, y^{\prime}, z\right)
\end{aligned}
$$

$$
\begin{aligned}
& +\left(2^{R_{2}}-1\right) \sum_{x, y^{\prime}, z} p\left(y^{\prime}\right) p(x) p(z \mid x) f^{Y}\left(x, y^{\prime}, z\right) \\
& +\left(2^{R_{1}}-1\right) \sum_{x^{\prime}, y, z} p\left(x^{\prime}\right) p(y) p(z \mid y) f^{X}\left(x^{\prime}, y, z\right) \\
& +\sum_{x, y, z} p(x) p(y) p(z \mid x, y)\left(\left(1-f^{X}(x, y, z)\right)\right. \\
& \left.+\left(1-f^{Y}(x, y, z)\right)+\left(1-f^{X, Y}(x, y, z)\right)\right) \\
& \stackrel{b}{\leq} \quad 2^{R_{1}+R_{2}} 2^{-I_{H}^{\epsilon}(X Y: Z)}+2^{R_{2}} 2^{-I_{H}^{\epsilon}(Y: X Z)}+2^{R_{1}} 2^{-I_{H}^{\epsilon}(X: Y Z)}+3 \epsilon \text {. } \\
& \text { Above, we use the property that } \\
& \begin{aligned}
f(x, y, z) \leq & \left\{f^{X}(x, y, z), f^{Y}(x, y, z), f^{X, Y}(x, y, z)\right\}, \\
1-f(x, y, z) \leq & \left(1-f^{X}(x, y, z)\right)+\left(1-f^{Y}(x, y, z)\right) \\
& +\left(1-f^{X, Y}(x, y, z)\right)
\end{aligned}
\end{aligned}
$$
for all triples $(x, y, z)$ in Step (a), and Equation 1 in Step (b). 